\title{
Cone-beam x-ray phase-contrast tomography for the observation of single cells in whole organs
}

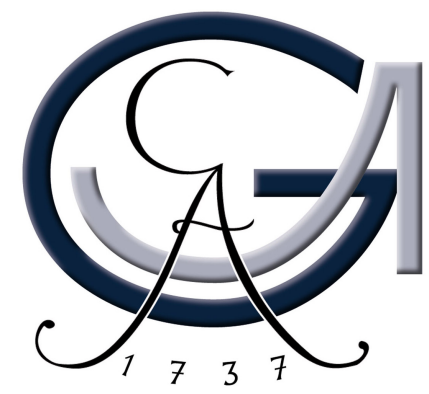

Dissertation

zur Erlangung des mathematisch-naturwissenschaftlichen

Doktorgrades

"Doctor rerum naturalium"

der Georg-August-Universität Göttingen

vorgelegt von

Martin Krenkel

aus Berlin

Göttingen, 2015

Institut für Röntgenphysik 


\section{Mitglieder des Betreuungsausschusses:}

Referent:

Prof. Dr. Tim Salditt

Institut für Röntgenphysik,

Georg-August-Universität Göttingen

1. Korreferent:

Prof. Dr. Dr. Detlev Schild

Abtl. Neurophysiologie und Zelluläre Biophysik,

Universitätsmedizin Göttingen

2. Korreferent:

PD Dr. Timo Aspelmeier

Institut für Mathematische Stochastik,

Georg-August-Universität Göttingen

\section{Tag der mündlichen Prüfung:}

22. Oktober 2015 




\section{Contents}

Introduction $\quad 1$

1 Concepts of x-ray propagation imaging 5

1.1 Propagation of electromagnetic waves . . . . . . . . . . . 6

1.1.1 Wave equations in vacuum ........... 6

1.1.2 Paraxial wave equation .................. 8

1.1.3 Angular spectrum approach . . . . . . . . . . . . 9

1.1.4 Huygens-Fresnel principle . . . . . . . . . . . . . 11

1.1.5 Numerical implementation of propagation . . . . . . . . 13

1.2 X-ray interactions with matter . . . . . . . . . . . 15

1.2.1 Wave equations in the presence of matter . . . . . . . 15

1.2.2 The refractive index .............. 16

1.2.3 Projection approximation ............... 17

1.3 From wave fields to images . . . . . . . . . . . . . . . 20

1.3.1 Contrast transfer function . . . . . . . . . . . . . 21

1.3.2 Transport of intensity equation . . . . . . . . . . . 23

1.3.3 Imaging regimes . . . . . . . . . . . . . . 25

1.3.4 Fresnel scaling theorem . . . . . . . . . . . . 27

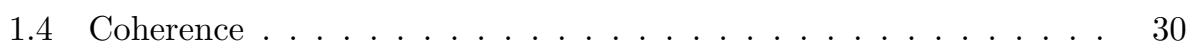

1.5 Dose and resolution . . . . . . . . . . . . . . 31

2 Phase-retrieval approaches $\quad 35$

2.1 Direct-contrast regime . . . . . . . . . . . . . . 36

2.1.1 Pure phase objects . . . . . . . . . . . . 36

2.1.2 Single material objects . . . . . . . . . . . . . . . 39

2.1.3 Bronnikov aided correction ............ 40

2.2 Holographic regime . . . . . . . . . . . . . . . . 43

2.2.1 Holographic reconstruction . . . . . . . . . . . . 43

2.2.2 CTF-based phase-retrieval . . . . . . . . . . 45

2.2 .3 Holo-TIE . . . . . . . . . . . . . . . 50

2.2.3.1 The ideal case . . . . . . . . . . . . . 50 
2.2.3.2 Treatment of noisy data . . . . . . . . . 53

2.2 .4 Iterative methods . . . . . . . . . . . . . . 56

2.2.4.1 Projection methods . . . . . . . . . . 56

2.2.4.2 Iterative reprojection phase-retrieval . . . . . . . 60

2.2.4.3 Iterative Newton methods . . . . . . . . . . . 62

3 Computed tomography $\quad 67$

3.1 Mathematical background ............... 67

3.1.1 Radon transform . . . . . . . . . . . . . . 67

3.1.2 Fourier slice theorem . . . . . . . . . . . 70

3.1.3 Filtered back projection ............. 71

3.1.4 Algebraic reconstruction technique . . . . . . . . . 73

3.1.5 Cone-beam reconstruction . . . . . . . . . . . 75

3.2 Artifacts in x-ray tomography . . . . . . . . . . . . 76

3.2.1 Ring artifacts and ring-removal . . . . . . . . . . 76

3.2 .2 Beam hardening . . . . . . . . . . . . 78

3.2 .3 Interior tomography . . . . . . . . . . . . . . . 79

3.2.4 Sampling artifacts ............... . . 81

3.2.5 Geometry artifacts ............... 82

3.2.6 Motion artifacts ................ . . 84

3.3 Alignment procedures and algorithms . . . . . . . . . . . 84

4 Experimental realization $\quad \mathbf{8 9}$

4.1 X-ray generation . . . . . . . . . . . . . . . 89

4.2 X-ray detectors . . . . . . . . . . . . . . . . . 91

4.2.1 Direct detectors . . . . . . . . . . . . . . . . 91

4.2 .2 Indirect detectors . . . . . . . . . . . . . . . . . 92

4.3 Laboratory setup JuLiA . . . . . . . . . . . . . . . . 96

4.4 Using synchrotron-radiation . . . . . . . . . . . . . . . . . . . 99

4.4.1 KB based setups at ID22 \& ID16a . . . . . . . . . . . . 99

4.4.2 The empty-beam problem . . . . . . . . . . . . 102

4.4 .3 X-ray waveguides . . . . . . . . . . . . . . . . . 104

4.4.4 Waveguide based setup GINIX . . . . . . . . . . 107

5 Tomographic imaging of the mouse lung 111

5.1 State of the art in small-animal imaging . . . . . . . . . . . . . 112

5.2 Methods . . . . . . . . . . . . . . . . . . 113

5.2.1 Sample preparation ............... 113 
5.2.2 Experimental setup and measurements . . . . . . . . . . 114

5.3 Results. . . . . . . . . . . . . . . . . 117

5.3.1 Phase-contrast in mouse lungs with laboratory sources . . . 117

5.3.2 The influence of the energy spectrum . . . . . . . . . . . 123

5.4 Summary and outlook . . . . . . . . . . . . . . . . 125

$6 \quad$ Single-cell imaging $\quad 127$

6.1 High resolution imaging of macrophages . . . . . . . . . . . . . . . 128

6.2 Methods . . . . . . . . . . . . . . . . . . . 129

6.2.1 Sample preparation . . . . . . . . . . . . 129

6.2.2 Staining procedures . . . . . . . . . . . . 132

6.2.3 Experimental parameters . . . . . . . . . . . . . 134

6.2.4 Waveguide-stripe removal . . . . . . . . . . . 136

6.32 D Imaging of single macrophages . . . . . . . . . . . . . . . . . . . . . . . . . . . . . . . . .

6.3.1 Dried macrophage cells . . . . . . . . . . . . 137

6.3.1.1 Single-distance phase-retrieval . . . . . . . . . . 138

6.3.1.2 Multi-distance phase-retrieval . . . . . . . . . . 142

6.3.2 Stained and unstained cells embedded in resin . . . . . . . 144

6.3.3 Towards in-vivo x-ray imaging of macrophages . . . . . . . 146

6.4 3D imaging of single macrophages . . . . . . . . . . . . . . . 151

6.4.1 Algorithmic motion correction . . . . . . . . . . 151

6.4.1.1 Vertical correction . . . . . . . . . . . . . 151

6.4.1.2 Horizontal correction . . . . . . . . . . . . 153

6.4.2 Stained macrophages in aqueous gels . . . . . . . . . . . 154

6.4.3 Cells embedded in resin . . . . . . . . . . . . . 157

6.4.3.1 Osmium stained . . . . . . . . . . . . . 157

6.4.3.2 Unstained cells . . . . . . . . . . . . 162

6.4.3.3 Osmium and barium stained ......... 163

6.5 Resolution of the 3D reconstructions . . . . . . . . . . . . . . 166

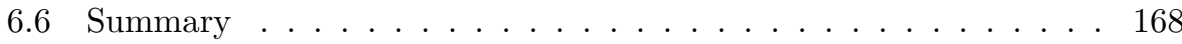

7 Tracking macrophages in the mouse lung $\quad 169$

7.1 The medically relevant question . . . . . . . . . . . . . . . . 169

7.2 Sample preparation . . . . . . . . . . . . . . . . . 171

7.3 Measurements and phase retrieval . . . . . . . . . . . . 173

7.4 Experimental results . . . . . . . . . . . . . . . . . . . . . . . 178

7.4.1 Phase-contrast imaging of asthmatic mouse lungs . . . . . . 178

7.4.2 Zoom tomography enabled by x-ray waveguides . . . . . . . 183 
7.4.3 Dose and resolution . . . . . . . . . . . . 188

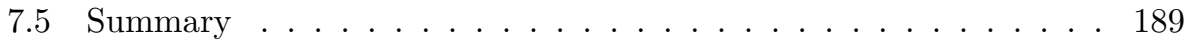

8 Conclusions $\quad 191$

$\begin{array}{ll}\text { Appendix } & 195\end{array}$

A.1 The Fourier transform and its properties . . . . . . . . . . . . . 195

A.2 Frechét derivative of the propagation operator . . . . . . . . . 196

A.3 List of Matlab functions . . . . . . . . . . . . . . . . . . . 197

A.3.1 Image preprocessing . . . . . . . . . . . . . . . . 198

A.3.2 Phase retrieval in the direct-contrast regime . . . . . . . . 199

A.3.3 Holographic phase-retrieval . . . . . . . . . . . . . . . 199

A.3.4 Functions useful for tomography . . . . . . . . . . . . . 200

$\begin{array}{ll}\text { Bibliography } & 202\end{array}$

$\begin{array}{lr}\text { Own publications } & 219\end{array}$

$\begin{array}{ll}\text { Danksagung } & 221\end{array}$

$\begin{array}{lr}\text { Lebenslauf } & \mathbf{2 2 5}\end{array}$ 


\section{Introduction}

For medical diagnostics, x-ray imaging is of great importance as the interactions with matter are so small that internal structures in oblique objects like the human body can be observed in a non-destructive way. Even shortly after the discovery of $\mathrm{x}$-rays in 1895, the technique was used to image bones inside the hand [150], paving the way for a new kind of medical examination. As x-ray images are projections, overlapping structures can not unambiguously be discerned in a single x-ray image. One method to overcome this limitation is computed tomography $(\mathrm{CT})$, in which several recordings under different angles are used to obtain a three-dimensional (3D) volume by means of numerical reconstruction using a computer [77]. In this way, the precise 3D location of internal structures is retrieved, which enables, e.g., the recognition of tumors and its treatment by radiation therapy [80]. In medical research, the precise location of single cells in relation to anatomical structures can help to understand fundamental biological processes. Data with sub-cellular resolution for, e.g., a whole animal would imply handling unreasonable amounts of data. Therefore, the ideal imaging technique should allow the observation of large samples for orientation with the possibility to zoom to specific regions of interest. In classical x-ray imaging the image formation is based on the partial absorption of the radiation, i.e. dense structures like the bones absorb more radiation than lighter elements comprising soft tissue. However, if structures with a weak absorption are observed, the transparency for x-rays may be limiting, as the absorption contrast may be insufficient to obtain a clear image. As x-rays are electromagnetic waves, not only their absorption but also the relative phase difference can carry information about an object. Over the past two decades, x-ray phase-contrast imaging has been developed, for which the underlying physical constants are up to 1000 times larger than for absorption imaging [124, 133]. In particular if very small structures on the micro- and nanometer-scale are of interest, low absorption coefficients become even more restrictive, as reasonable absorption levels build up only over longer path lengths [89]. Thus, phase contrast is an essential tool, to obtain sufficient contrast for high-resolution hard x-ray imaging of biomedical specimen.

There are several ways of implementing x-ray phase-contrast, like Zernike phase- 
contrast in zone-plate microscopy [34, 156], grating interferometry [39, 111, 138], scanning diffraction microscopy $[41,167]$ and phase contrast based on coherent free-space propagation behind the sample [37, 125, 185]. Each method has its advantages and drawbacks and can be applied depending on the length scales and applications [99, 193]. For example, if Zernike phase-contrast in zone-plate based microscopes is utilized, a resolution down to some ten nanometers has been achieved in 2D [99], which however can not be achieved on specimen exceeding a thickness of about 300 micron [34]. Grating based phase-contrast imaging offers a very high sensitivity [193] and is compatible with low-brilliance laboratory sources [138], but the best resolution achieved so far is in the range of some micrometers [139], limited to the quality of the necessary gratings. Additionally, the need for optical elements behind the sample increases the radiation dose and imperfections of these elements may limit the quality of the reconstructions.

Coherent lensless imaging techniques are potentially most dose efficient, as no optical element behind the sample is needed for the image formation. In scanning transmission x-ray microscopy (STXM) with ptychographic phase-retrieval and in coherent diffractive imaging (CDI) resolutions down to $10 \mathrm{~nm}$ have been achieved for strongly diffracting test-structures [122, 158, 186] and to about $50 \mathrm{~nm}$ on biological specimen [184]. Successful high-resolution reconstructions have been demonstrated in 3D on the organelle [122] and single cell level [82, 119]. However, the typically very small beam size and the scanning overhead are a major drawback of these methods. The time required to perform a typical ptychographic tomography measurement is in the order of 10 hours [61], which limits the usefulness of this method for specimen with a size of several $100 \mu \mathrm{m}$.

Propagation-based phase-contrast enables a lensless full-field imaging approach compatible with a wide range of experimental parameters. In particular if a magnifying cone-beam illumination is employed, the field of view (FOV) and resolution can be tuned by changing the geometric magnification. The approach is conceptually simple: The standard radiographic exposure is extended by a free-space propagation and enhanced coherence properties of the illumination . Based on self-interference of the radiation, an in-line hologram can be recorded, which depending on the actual propagation distance still shows resemblance to the original object. Especially for small propagation distances, the approach is also compatible with partially coherent laboratory sources $[15,132]$. The main difficulty in propagation imaging is the phase-retrieval step, which serves to provide quantitative information about the specimen, needed e.g. for tomographic applications. Typically the phase retrieval is based on additional information about the object like 
measurements in several planes $[2,37]$ or a priori assumptions to the object (e.g. weak absorption, compact support or positivity of physical constants $[17,54,151])$ which also can be combined [88].

To enable 3D imaging of entire organs at large fields of view and of regions of interest at nanoscale resolution with the same technique, propagation-based phasecontrast imaging is the method of choice. Using this approach, reconstructions of whole organs with a low-resolution in the range of some micrometer $[15,126]$ as well as reconstructions of isolated cells with a high-resolution below $50 \mathrm{~nm}$ have been demonstrated $[16,17]$. However, to provide a tool for biomedical research, the question arises if high-resolution reconstructions can also be obtained for very large samples. In particular, the observation of cells in relation to anatomical structures in their native environment can help to answer many biomedically relevant questions. The goal of the present work is to investigate if propagation-based phase-contrast can be used to obtain 3D structural information with sub-cellular resolution in whole organs.

As the lung with its intricate structure is an ideal example how the 3D structure enables a biological function, different samples ranging from whole mice over thick lung-tissue slices down to single alveolar cells (macrophages) are used as specimens for proof-of-concept demonstrations in this work. Although macrophages are known to be involved in processes of allergic asthma [114], their exact role is still not well understood $[10,11,43,190]$. Not only it is interesting whether single cells inside large surrounding tissues can be observed, but an important question is if macrophages are able to migrate through epithelial cells inside the lung.

To provide a deeper insight and to answer the raised questions, the present thesis is organized as follows: After a brief introduction of the necessary concepts of Fourier optics, free-space propagation and image formation in chapter 1, several phase-retrieval approaches are presented, which provide a tool to obtain quantitative projection images from measured data. In chapter 3 the fundamentals of computed tomography are presented and typical artifacts that may occur in tomographic imaging and their treatments are embraced. A large part of this work was the experimental realization and improvement of existing setups for propagation imaging, so that in chapter 4 a brief summary of the latest synchrotron-based and laboratory setups is given. Experimental applications of propagation based phasecontrast tomography to biomedical specimens are presented in chapter 5, where micrometer-scale 3D reconstructions of whole mouse lungs are obtained with a laboratory source. To achieve reconstructions with nanoscale resolution, following experiments are performed on synchrotron storage rings. 3D reconstructions of 
isolated cells are presented at a resolution down to $50 \mathrm{~nm}$ and the influence of different contrast agents is investigated in chapter 6. Finally, the question about migration properties of macrophages in mouse lungs is addressed in chapter 7, using high-resolution phase-contrast measurements on thick mouse lung-tissue slices. The thesis is closed with a short conclusion in chapter 8 , where further experiments are proposed. 


\section{Concepts of x-ray propagation imaging}

A generic propagation-imaging experiment can be described by the sketch in Fig. 1.1. First, a well-defined, divergent x-ray beam is produced that illuminates the sample. The sample modifies the beam and after a finite propagation distance $z_{2}$, the phase-shift introduced by the object can be measured as it will transform into intensity variations.

This chapter will deal with the theory needed to understand the concept of propagation-based phase-contrast tomography. Using the formalism of classical electrodynamics and Fourier optics, wave propagation will be explained as an essential tool to understand the formation of a measurable intensity image, which plays a crucial role in the derivation and application of phase-retrieval algorithms. A large part of this work was the implementation and evaluation of different approaches, so that chapter 2 will give a detailed overview of available phase-retrieval approaches. Following the introduction of the different propagation concepts, pos-

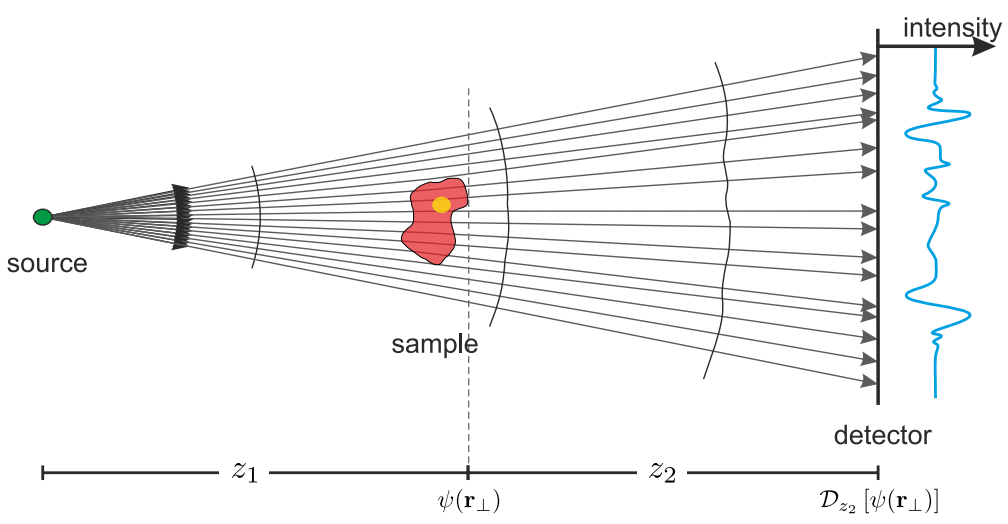

Figure 1.1: Sketch of the generic propagation-based phase-contrast setup: A well-defined, divergent $x$-ray beam, emerging from a source, propagates over a distance $z_{1}$ to the object plane. The object will change the phase distribution of the illuminating beam, leading to a disturbed wave-front. At a propagation distance $z_{2}$ the phase changes are transformed into intensity changes, which can be measured by a detector. 
sible interactions between x-ray radiation and biological samples are discussed. The projection approximation will be introduced that simplifies the interaction of object and illumination to a single plane, the so-called exit plane behind the object. Equipped with these tools, the formation of a propagation-based phasecontrast image can be described, for which the contrast transfer function plays an important role. The different imaging regimes used for experiments in this work are presented using numerical simulations, showing advantages and challenges for the application to relevant samples.

\subsection{Propagation of electromagnetic waves}

\subsubsection{Wave equations in vacuum}

At the beginning of the 20th century it was under discussion if x-rays behave like the previously discovered electromagnetic waves or if they can be fully described by rays without wave properties. Laue could experimentally show that x-rays are indeed electromagnetic waves [50] and therefore have to obey Maxwell's equations. In vacuum ${ }^{1}$ these can be written as follows $[25,133]$ :

$$
\begin{aligned}
\nabla \cdot \mathbf{E}(\mathbf{r}, t) & =0, \\
\nabla \cdot \mathbf{B}(\mathbf{r}, t) & =0, \\
\nabla \times \mathbf{E}(\mathbf{r}, t)+\partial_{t} \mathbf{B}(\mathbf{r}, t) & =\mathbf{0}, \\
\nabla \times \mathbf{B}(\mathbf{r}, t)-\epsilon_{0} \mu_{0} \partial_{t} \mathbf{E}(\mathbf{r}, t) & =\mathbf{0} .
\end{aligned}
$$

Here $\mathbf{E}$ is the electric field and $\mathbf{B}$ the magnetic induction dependent on the threedimensional (3D) coordinate vector $\mathbf{r}=(x, y, z)^{T} \in \mathbb{R}^{3}$ and the time $t \in \mathbb{R}$. The partial derivative in terms of $t$ is denoted by $\partial_{t}$, and $\nabla=\left(\partial_{x}, \partial_{y}, \partial_{z}\right)^{T}$ denotes the gradient operator, so that $\nabla$. is the divergence and $\nabla \times$ the curl operator. $\epsilon_{0}=8.854 \cdot 10^{-12} \mathrm{AsV}^{-1} \mathrm{~m}^{-1}$ is the electric and $\mu_{0}=4 \pi \cdot 10^{-7} \mathrm{VsA}^{-1} \mathrm{~m}^{-1}$ the magnetic field constant. The Maxwell equations describe the free-space evolution of a general electromagnetic field in space and time. We allow the solutions to these equations to be complex, being aware of the fact that only the real part of a quantity has a physical meaning. By using the Grassman vector identity two

${ }_{1}$ Note that SI units are used here and throughout the thesis and vector quantities are indicated using bold letters. 
independent wave equations can be derived using Eq. (1.1) to (1.4)

$$
\begin{aligned}
& \left(\epsilon_{0} \mu_{0} \partial_{t}^{2}-\nabla^{2}\right) \mathbf{E}(\mathbf{r}, t)=0 \\
& \left(\epsilon_{0} \mu_{0} \partial_{t}^{2}-\nabla^{2}\right) \mathbf{B}(\mathbf{r}, t)=0
\end{aligned}
$$

Equations (1.5) and (1.6) state that each component of the electric field and magnetic induction has to obey a wave equation of similar form, which motivates the introduction of a single scalar wave equation, also known as the d'Alembert equation

$$
\left(\frac{1}{c^{2}} \partial_{t}^{2}-\nabla^{2}\right) \Psi(\mathbf{r}, t)=0 .
$$

Here $\Psi$ may be any component of the electric field $\mathbf{E}$ or the magnetic induction $\mathbf{B}$, and the definition of the speed of an electromagnetic wave in vacuum $c=1 / \sqrt{\epsilon_{0} \mu_{0}}$ is introduced. One special solution to this differential equation is given by a monochromatic plane wave

$$
\Psi^{(\mathrm{PW})}(\mathbf{r}, t)=\mathrm{e}^{(\mathrm{ik} \cdot \mathbf{r}-\omega t)},
$$

if the wave vector $\mathbf{k}=\left(k_{x}, k_{y}, k_{z}\right)^{T} \in \mathbb{R}^{3}$, with modulus $|\mathbf{k}|=: k=2 \pi / \lambda$ and $\lambda$ being the wavelength, fulfills $k=\omega / c$. In these waves, points with the same phase travel with velocity $c$, which was experimentally determined to be the speed of light and therefore led to the discovery that light is an electromagnetic wave [133]. In order to find a time-independent equation let us consider an arbitrary wave field $\boldsymbol{\Psi}(\mathbf{r}, t)$ that can be described by its spectral Fourier expansion ${ }^{2}$

$$
\Psi(\mathbf{r}, t)=\frac{1}{\sqrt{2 \pi}} \int_{0}^{\infty} \psi_{\omega}(\mathbf{r}) \mathrm{e}^{-\mathrm{i} \omega t} \mathrm{~d} \omega .
$$

This decomposition can be understood such that the wave field $\Psi(\mathbf{r}, t)$ consists of monochromatic waves that are weighted with $\psi_{\omega}(\mathbf{r})$. We insert Eq. (1.9) into the d'Alembert wave-equation (1.7), change the order of integration and differentiation and perform the time derivative, yielding

$$
\int_{0}^{\infty}\left[\left(\nabla^{2}+\frac{\omega^{2}}{c^{2}}\right) \psi_{\omega}(\mathbf{r})\right] \mathrm{e}^{-\mathrm{i} \omega t} \mathrm{~d} \omega=0 .
$$

To fulfill Eq. (1.10) for general $\psi_{\omega}(\mathbf{r})$, the term in square brackets has to vanish.

\footnotetext{
2 Note that negative frequencies are ignored here, resulting in "a certain useful analyticity" [133].
} 
This condition for the time-independent part $\psi_{\omega}(\mathbf{r})$ is known as the Helmholtz equation $[133]$

$$
\left(\nabla^{2}+k^{2}\right) \psi_{\omega}(\mathbf{r})=0
$$

with $k=\omega / c$ as in the case of plane waves. To obtain solutions that are not monochromatic, $\omega$-dependent solutions $\psi_{\omega}(\mathbf{r})$ can be put into the ansatz of spectral decomposition (1.9). For the sake of convenience we will ignore the subscript $\omega$ in the following equations.

Analogously to the time-dependent plane waves $\Psi^{(\mathrm{PW})}(\mathbf{r}, t)$, which are solutions to the d'Alembert equation (1.7), one can find monochromatic stationary plane waves $\psi^{(\mathrm{PW})}(\mathbf{r})$ to be solutions to the Helmholtz equation

$$
\psi^{(\mathrm{PW})}(\mathbf{r})=\mathrm{e}^{\mathrm{i} \mathbf{k} \cdot \mathbf{r}}=\mathrm{e}^{\mathrm{i}\left(k_{x} x+k_{y} y+k_{z} z\right)},
$$

where the wave vector $\mathbf{k}$ determines the propagation direction. Other fundamental solutions are given by time-independent spherical waves

$$
\psi^{(\mathrm{SW})}(\mathbf{r})=\frac{1}{\left|\mathbf{r}-\mathbf{r}_{0}\right|} \mathrm{e}^{\mathrm{i} k\left|\mathbf{r}-\mathbf{r}_{0}\right|},
$$

for $\mathbf{r} \neq \mathbf{r}_{0}$. These waves do not have a distinct propagation direction. Instead, concentric spheres of constant phase around an origin $\mathbf{r}_{0}$ will propagate equally in all directions.

\subsubsection{Paraxial wave equation}

A way to further simplify the wave equation is to use the so-called small-angle, Fresnel or paraxial approximation. A wave is called paraxial, if the normal vectors of the wavefront form small angles with respect to a given optical axis. This is a good description for "beam-like" waves that propagate in one direction and thus vary mainly perpendicular to that direction [133]. To derive the paraxial wave equation we start with the separation ansatz

$$
\psi(\mathbf{r})=\psi^{\prime}(\mathbf{r}) \cdot \mathrm{e}^{\mathrm{i} k z}
$$

where, without loss of generality, we define the $z$-axis to be the optical axis. This wave consists of a fast oscillating plane-wave term $\mathrm{e}^{\mathrm{i} k z}$ along the optical axis and an envelope $\psi^{\prime}(\mathbf{r})$ that changes slowly along the optical axis. Inserting the ansatz (1.14) into the Helmholtz equation (1.11), performing the derivative and dividing 
all terms by $\mathrm{e}^{\mathrm{i} k z}$ yields

$$
\left(\nabla_{\perp}^{2}+\partial_{z}^{2}+2 \mathrm{i} k \partial_{z}\right) \psi^{\prime}(\mathbf{r})=0
$$

Here the Laplacian $\nabla^{2}$ is split up into the lateral part $\nabla_{\perp}^{2}:=\partial_{x}^{2}+\partial_{y}^{2}$ and the axial part $\partial_{z}^{2}$. The paraxial approximation manifests itself in neglecting the second derivative $\partial_{z}^{2} \psi^{\prime}(\mathbf{r})$. The resulting equation is called the homogenous paraxial equation

$$
\left(\nabla_{\perp}^{2}+2 \mathrm{i} k \partial_{z}\right) \psi^{\prime}(\mathbf{r})=0 .
$$

One simple solution to this equation is given by a so-called parabolic beam

$$
\psi^{(\mathrm{PB})}(\mathbf{r})=\frac{1}{z} \mathrm{e}^{\mathrm{i} k \mathbf{r}_{\perp}^{2} / 2 z},
$$

where $\mathbf{r}_{\perp}=(x, y)^{T}$ is introduced. This solution can be obtained by assuming a spherical wave originating at $\mathbf{r}=\mathbf{0}$ and performing a small-angle approximation for the modulus of $\mathbf{r}$ [14]. Another important solution is the Gaussian beam, described in detail e.g. in [182]. It can be used to approximate beams exiting $\mathrm{x}$-ray waveguides [90], which play an important role in this thesis.

\subsubsection{Angular spectrum approach}

Let us consider a solution to the Helmholtz equation $\psi\left(\mathbf{r}_{\perp}, z\right)$ known in a plane perpendicular to the optical axis at position $z$. To understand how this field will change during propagation along the optical axis, we decompose it in lateral Fourier-components

$$
\psi\left(\mathbf{r}_{\perp}, z\right)=\frac{1}{2 \pi} \int_{\mathbb{R}^{2}} \tilde{\psi}\left(\mathbf{k}_{\perp}, z\right) e^{\mathrm{i} \mathbf{k}_{\perp} \mathbf{r}_{\perp}} d \mathbf{k}_{\perp}
$$

with $\mathbf{k}_{\perp}=\left(k_{x}, k_{y}\right)$ and $\tilde{\psi}\left(\mathbf{k}_{\perp}, z\right)$ denoting the lateral Fourier transform of $\psi\left(\mathbf{r}_{\perp}, z\right)$. In the following, Fourier-transformed quantities will be marked with a tilde $(\sim)$. Inserting Eq. (1.18) into the Helmholtz equation (1.11) yields a second-order, scalar differential equation in terms of $z$ for $\tilde{\psi}\left(\mathbf{k}_{\perp}, z\right)$. This initial value problem can be solved to yield the general solution [55]

$$
\tilde{\psi}\left(\mathbf{k}_{\perp}, z_{1}\right)=\tilde{\psi}\left(\mathbf{k}_{\perp}, z_{0}\right) e^{\mathrm{i} \Delta z \sqrt{k^{2}-k_{\perp}^{2}}}
$$


with $k_{\perp}^{2}=\left|\mathbf{k}_{\perp}\right|^{2}=k_{x}^{2}+k_{y}^{2}, \Delta z=z_{1}-z_{0}$ and $z_{1}>z_{0}$. Using this solution Eq. (1.18) reads

$$
\psi\left(\mathbf{r}_{\perp}, z_{1}\right)=\frac{1}{2 \pi} \int_{\mathbb{R}^{2}} \tilde{\psi}\left(\mathbf{k}_{\perp}, z_{0}\right) e^{\mathrm{i} \Delta z \sqrt{k^{2}-k_{\perp}^{2}}} e^{i \mathbf{k}_{\perp} \mathbf{r}} d \mathbf{k}_{\perp} .
$$

This equation conveys how to propagate an arbitrary wave field, known at a distance $z_{0}$, to another distance $z_{1}=z_{0}+\Delta z$. Note that the propagation is only dependent on $\Delta z$ and that the absolute value of $z_{0}$ does not matter. Hence, we will set $z_{0}=0$ in the following and regard Eq. (1.20) as a rule to propagate a given field by a relative distance $\Delta z=z-0=z$. With this convention and with the operator $\mathcal{F}_{\perp}$ denoting the lateral Fourier transform, Eq. (1.20) can be written as

$$
\psi\left(\mathbf{r}_{\perp}, z\right)=\mathcal{F}_{\perp}^{-1}\left[e^{\mathrm{i} z \sqrt{k^{2}-k_{\perp}^{2}}} \mathcal{F}_{\perp}\left(\psi\left(\mathbf{r}_{\perp}, 0\right)\right)\right] .
$$

Equation (1.21), also known as the free-space propagator [133], states that for the propagation of a wave field over a distance $z$, the initial wave field is laterally Fourier transformed, multiplied with a complex phase factor and back transformed to real space. In particular, the lateral dimensions defined by $\mathbf{r}_{\perp}$ do not change, so this propagator is well suited for near-field propagation. ${ }^{3}$

Note that for $k_{\perp}^{2}>k^{2}$ the square-rooted term in Eq. (1.21) is negative. Hence, the exponential term will be real and the wave will be damped out within a range of a few wavelengths instead of being an oscillating, propagating wave. In this case one speaks of an evanescent wave. If there are no evanescent waves, the operator defined by Eq. (1.21) is unitary [112] and the equation can be used for $z<0$ to perform a back propagation, also known as inverse diffraction [160].

For experiments in this work, the x-ray beams are well described by paraxial beams. Therefore, we now derive a paraxial operator in analogy to Eq. (1.21). For the wave vector of such a beam it is [53]

$$
k_{\perp}^{2} \ll k^{2} .
$$

This motivates the Taylor expansion of the phase factor in Eq. (1.21)

$$
\sqrt{k^{2}-k_{\perp}^{2}}=k-\frac{k_{\perp}^{2}}{2 k}+\mathcal{O}\left(k_{\perp}^{4}\right) .
$$

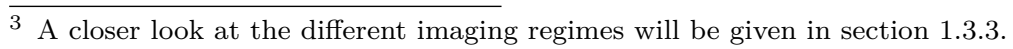


After omitting the terms $\mathcal{O}\left(k_{\perp}^{4}\right)$ Eq. (1.21) reads

$$
\psi\left(\mathbf{r}_{\perp}, z\right)=\mathcal{D}_{z}\left[\psi\left(\mathbf{r}_{\perp}, 0\right)\right]:=e^{\mathrm{i} k z} \cdot \mathcal{F}_{\perp}^{-1}\left[e^{-\mathrm{i} \frac{z k_{\perp}^{2}}{2 k}} \mathcal{F}_{\perp}\left(\psi\left(\mathbf{r}_{\perp}, 0\right)\right)\right] .
$$

This equation describes the so-called Fresnel diffraction [133]. We introduce the Fresnel propagator $\mathcal{D}_{z}$ that propagates a given field over a distance $z$ in the paraxial approximation. Note that the fast oscillating term $\mathrm{e}^{\mathrm{i} k z}$, which is occurring in Eq. (1.24), was separated from solutions to the paraxial wave equation (1.16). As it only gives a constant phase offset for planes perpendicular to the propagation axis, it will be ignored in the numerical implementation of Eq. (1.24).

\subsubsection{Huygens-Fresnel principle}

Another direct solution to the Helmholtz equation (1.11) can be found with the formalism of Green's functions, leading to the Kirchhoff and the Rayleigh-Sommerfeld theories, as described in detail in [55]. The first Rayleigh-Sommerfeld solution can be expressed as

$$
\psi\left(\mathbf{r}_{\perp, 1}, z\right)=\frac{1}{\mathrm{i} \lambda} \int_{\mathbb{R}^{2}} \psi\left(\mathbf{r}_{\perp, 0}, 0\right) \frac{\mathrm{e}^{\mathrm{i} k r_{01}}}{r_{01}} \cos \theta \mathrm{d} \mathbf{r}_{\perp, 0}
$$

with the wavelength $\lambda$, the wave field in the initial plane $\psi\left(\mathbf{r}_{\perp, 0}, 0\right)$ and the propagated wave field $\psi\left(\mathbf{r}_{\perp, 1}, z\right)$ in a plane with axial distance $z$. Other geometric quantities are depicted in Fig. 1.2 and by using Pythagoras' theorem the distance $r_{01}$ is determined by

$$
r_{01}=\sqrt{\left(x_{1}-x_{0}\right)^{2}+\left(y_{1}-y_{0}\right)^{2}+z^{2}} .
$$

Equation (1.25) is the mathematical formulation of the well-known HuygensFresnel principle. Upon illumination of the initial plane with electromagnetic radiation, each point in this plane can be seen as a secondary source of spherical waves. Thus, the wave function in a second plane with distance $z$ can be composed as a superposition of spherical waves $\mathrm{e}^{\mathrm{i} k r_{01}} / r_{01}$ weighted with the wave function in the originating plane at $z=0$ and a direction dependent term $\cos \theta$ accounting for the vectorial summation. The complex prefactor is a result of the exact derivation that can be found, e.g., in [55].

Let us again consider the special case of a paraxial wave. It states that a propagating beam mainly forms small angles with respect to the optical axis. This 


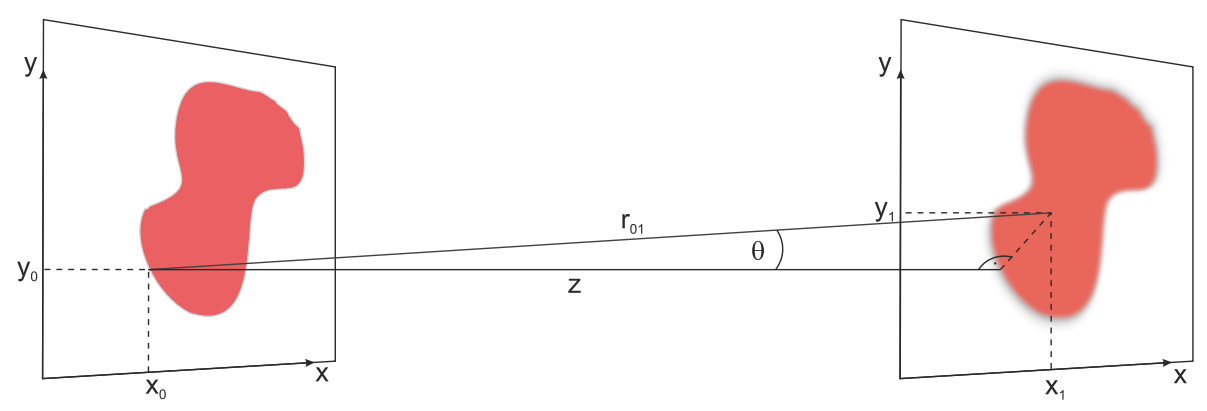

Figure 1.2: Illustration of the Huygens-Fresnel principle: The complex wavefunction at the point $\mathbf{r}_{\perp, 1}=\left(x_{1}, y_{1}\right)$ with axial position $z$ can be described as the superposition of spherical waves, originating in the plane $z=0$ and weighted with the initial wave function $\psi\left(\mathbf{r}_{\perp, 0}\right)$. The vector $\mathbf{r}_{01}$ forms the angle $\theta$ with the optical axis and its length $r_{01}$ is the distance, each superimposed wave has to travel.

means in particular that the maximum lateral deviations

$$
\left|\Delta \mathbf{r}_{\perp}\right|^{2}=\left|\mathbf{r}_{\perp, 1}-\mathbf{r}_{\perp, 0}\right|^{2}=\left(x_{1}-x_{0}\right)^{2}+\left(y_{1}-y_{0}\right)^{2}
$$

are small in comparison to the propagation distance $z$. Hence we can approximate $\cos \theta$ and the distance $r_{01}$ as follows

$$
\begin{aligned}
\cos \theta & =1+\mathcal{O}\left(\theta^{2}\right), \\
r_{01} & =z+\frac{\Delta \mathbf{r}_{\perp}^{2}}{2 z}+\mathcal{O}\left(\Delta \mathbf{r}_{\perp}{ }^{4}\right) .
\end{aligned}
$$

For the radial decay $1 / r_{01}$ we use the first-order approximation of $r_{01}$, whereas for $\mathrm{e}^{\mathrm{i} k r_{01}}$ we take the third-order approximation, as deviations have a larger influence in the exponential function. With these approximations, Eq. (1.25) reads

$$
\psi\left(\mathbf{r}_{\perp, 1}, z\right)=\frac{1}{\mathrm{i} \lambda} \frac{e^{\mathrm{i} k z}}{z} \int_{\mathbb{R}^{2}} \psi\left(\mathbf{r}_{\perp, 0}, z\right) \mathrm{e}^{\frac{\mathrm{i} k}{2 z}\left(\mathbf{r}_{\perp, 1}-\mathbf{r}_{\perp, 0}\right)^{2}} \mathrm{~d} \mathbf{r}_{\perp, 0}
$$

This equation is the real-space formulation ${ }^{4}$ of the Fresnel propagation operator $\mathcal{D}_{z}$, derived with the angular spectrum approach in Eq. (1.24), in which the propagation is now formulated as a convolution of the initial wave field with the

${ }^{4}$ Note that in [55] a different definition of the Fourier transform than in [133] was used (see appendix A.1), which has to be accounted for if the filter kernel in Fourier space is to be directly calculated by the Fourier transform of Eq. (1.31). 
convolution kernel

$$
H(x, y)=\mathrm{e}^{\frac{\mathrm{i} k}{2 z}\left(x^{2}+y^{2}\right)} .
$$

To distinguish some special cases, we expand the bracketed term in the exponent of Eq. (1.30), reorder the terms and summarize the prefactors to $C:=\frac{\mathrm{e}^{\mathrm{i} k z}}{\mathrm{i} \lambda z} \mathrm{e}^{\frac{\mathrm{i} k}{2 z} r_{\perp, 1}^{2}}$ yielding

$$
\psi\left(\mathbf{r}_{\perp, 1}, z\right)=C \int_{\mathbb{R}^{2}} \psi\left(\mathbf{r}_{\perp, 0}, 0\right) \mathrm{e}^{\frac{\mathrm{i} k}{2 z} r_{\perp, 0}^{2}} \mathrm{e}^{-\frac{\mathrm{i} k}{z}\left(\mathbf{r}_{\perp, 0} \cdot \mathbf{r}_{\perp, 1}\right)} \mathrm{d} \mathbf{r}_{\perp, 0}
$$

The above equation states that the propagated field can be written as the lateral Fourier transform of the term

$$
\psi\left(\mathbf{r}_{\perp, 0}, 0\right) \mathrm{e}^{\frac{\mathrm{i} k}{2 z} r_{\perp, 0}^{2}}
$$

In particular if the phase-factor $\mathrm{e}^{\frac{\mathrm{i} k}{2 z}} r_{\perp, 0}^{2}$ can be neglected, the propagated field is the Fourier transform of the initial wave field, known in the literature as Fraunhofer diffraction [133]. With $k=2 \pi / \lambda$, this justifies the definition of the so-called Fresnel number

$$
F:=\frac{a^{2}}{\lambda z}
$$

where $a$ describes the size of a typical lateral structure. Thus, the Fraunhofer regime will be reached as long as $F \ll 1$ holds for the largest structure present in an image. For $F \approx 1$ Fresnel diffraction will occur and the Fresnel number can be used to distinguish different imaging regimes as described in section 1.3.3. Note that in the formulation of Eq. (1.32) the lateral distances change during propagation if it is implemented via a discrete Fourier transform. Especially for the Fraunhofer regime this means that besides a geometric magnification the farfield does not change during propagation.

\subsubsection{Numerical implementation of propagation}

So far different equations to propagate a known solution $\psi(x, y, 0)$ to another plane on the optical axis $z$ yielding $\psi(x, y, z)$ have been presented. Let us again consider a solution to the paraxial wave equation (1.16) that can be propagated using for example Eq. (1.24) or Eq. (1.32). Both equations are mathematically equivalent but, however, can be used for different imaging regimes to model numerical propagation.

For a numerical implementation the fast Fourier transform (FFT) [142] can be used to calculate the analytical Fourier transforms occurring in Eq. (1.24) and (1.24). If one of the equations is implemented via the FFT, one has to consider 
proper sampling of the phase factor [177]. For the numerical solutions, wave fields are given on a discrete grid of pixels. With a given pixel size $p_{x}$ in real space and $\Delta k_{x}$ in reciprocal space the reciprocity relation

$$
p_{x} \Delta k_{x}=\frac{2 \pi}{N_{x}}
$$

holds. Here, $N_{x}$ denotes the number of pixels. This equation is analogously true for $p_{y}, \Delta k_{y}$ and $N_{y}$. The Fresnel propagator (1.24) contains two Fourier transforms and needs for the phase term to be sampled in Fourier space. Thus, Nyquist sampling requires [53]

$$
\frac{\lambda z}{N_{x} p_{x}^{2}} \leq 1
$$

In other words this equation states that for a given wavelength $\lambda$ and discretization $p_{x}$ and $N_{x}$, Eq. (1.24) is only valid up to a certain distance $z$. Hence, the propagator defined by Eq. (1.24) is called the "near-field Fresnel propagator". For Eq. (1.32) the phase factor has to be sampled in real space, which analogously leads to the condition

$$
\frac{\lambda z}{N_{x} p_{x}^{2}} \geq 1
$$

Thus Eq. (1.32) can be called the "far-field Fresnel propagator". Note that in both cases the Fresnel number with a lateral size of one pixel occurs

$$
F_{x}^{1}=\frac{p_{x}^{2}}{\lambda z}
$$

It is remarkable that, not only for the sampling but also for the propagation and phase-retrieval approaches, the exact values of $\lambda, z$ and $p_{x}$ do not matter under paraxial conditions. Instead, it is always the combination of these three in a form of the Fresnel number that affects the physical properties of a wave field.

All experiments relevant for this thesis are performed in a way that the nearfield Fresnel propagator is a good description of the underlying physical concepts, so that we will concentrate on it in the following. To ensure Nyquist sampling for larger distances than that defined by Eq. (1.36), it is possible to increase the number of pixels $N_{x}$. The enlargement can be performed by replicating the border values of a discrete representation, which effectively increases the sampling in Fourier space and also avoids non-physical edge effects. 


\section{$1.2 \mathrm{X}$-ray interactions with matter}

So far we have derived mathematical tools to propagate a known wave field over a distance $z$ in vacuum, which can be used as a good approximation for air. However, to reveal properties of biomedical samples, interactions between x-rays and matter need to be considered. This chapter will briefly deal with the wave equations in the presence of matter, leading to the projection approximation, which allows describing the interaction between x-rays and weak objects in a simplified way. Within this approximation all interactions are described by a complex exit wave that can be propagated in free space with the tools derived before.

\subsubsection{Wave equations in the presence of matter}

In the most general case the Maxwell equations can be written as [79]

$$
\begin{aligned}
\nabla \cdot \mathbf{D}(\mathbf{r}, t) & =\rho(\mathbf{r}, t), \\
\nabla \cdot \mathbf{B}(\mathbf{r}, t) & =0, \\
\nabla \times \mathbf{E}(\mathbf{r}, t)+\partial_{t} \mathbf{B}(\mathbf{r}, t) & =0, \\
\nabla \times \mathbf{H}(\mathbf{r}, t)-\partial_{t} \mathbf{D}(\mathbf{r}, t) & =\mathbf{j}(\mathbf{r}, t) .
\end{aligned}
$$

Here, additionally to the terms known from chapter 1.1, the electric displacement $\mathbf{D}(\mathbf{r}, t)=\epsilon \mathbf{E}(\mathbf{r}, t)$, the magnetic field $\mathbf{H}(\mathbf{r}, t)=\mathbf{B}(\mathbf{r}, t) / \mu$, the charge density $\rho(\mathbf{r}, t)$ and the current density $\mathbf{j}(\mathbf{r}, t)$ are introduced. In general $\epsilon$ and $\mu$ could be tensors of second order, but we restrict considerations to linear isotropic materials in which $\epsilon$ and $\mu$ become scalar quantities. Most biologically relevant samples are, moreover, non-magnetic which allows the assumption $\mu=\mu_{0}$. Additionally, we will only consider static materials, i.e., $\epsilon(\mathbf{r}, t)=\epsilon(\mathbf{r})$ and we assume the absence of charge or current densities and that polarization effects can be neglected. With these assumptions, a scalar wave equation can be derived with a similar strategy as in section 1.1.1, yielding

$$
\left(\epsilon(\mathbf{r}) \mu_{0} \partial_{t}^{2}-\nabla^{2}\right) \Psi(\mathbf{r}, t)=0 .
$$

With the definition of the refractive index

$$
n(\mathbf{r})=\sqrt{\epsilon(\mathbf{r}) / \epsilon_{0}}
$$


and a time-separation ansatz as in section 1.1.1, the inhomogeneous Helmholtz equation can be derived [133]

$$
\left(\nabla^{2}+k^{2} n(\mathbf{r})^{2}\right) \psi(\mathbf{r})=0
$$

\subsubsection{The refractive index}

For x-rays the refractive index is very close to unity and therefore it is often written as $[3]$

$$
n(\mathbf{r})=1-\delta(\mathbf{r})+\mathrm{i} \beta(\mathbf{r}),
$$

with the decrement $\delta(\mathbf{r})$ and the extinction coefficient $\beta(\mathbf{r})$. To gain an understanding for typical values for $\delta(\mathbf{r})$ and $\beta(\mathbf{r})$, we consider a generic biological material that can be described with the chemical formula $\mathrm{H}_{50} \mathrm{C}_{30} \mathrm{~N}_{9} \mathrm{O}_{10} \mathrm{~S}_{1}$ and a mass density of $1.35 \mathrm{~g} / \mathrm{cm}^{3}$ [78]. For a typical energy used in this thesis $(E=13.8 \mathrm{keV})$ the values are [71]

$$
\begin{aligned}
& \delta \approx 1.57 \cdot 10^{-6}, \\
& \beta \approx 1.91 \cdot 10^{-9},
\end{aligned}
$$

so $\delta$ is three orders of magnitude larger than $\beta$. The energy $E$ of electromagnetic waves can be linked to the quantities introduced before [3]

$$
E[\mathrm{keV}]=\frac{h c}{\lambda}=\frac{12.398}{\lambda[\AA]},
$$

with the Planck constant $h \simeq 6.626 \cdot 10^{-34} \mathrm{Js}$, the wavelength $\lambda$ and the vacuum wave velocity $c$.

The interactions between x-rays and matter can be mainly attributed to bound electrons of a material. Away from so-called absorption edges, which are caused by resonance effects, the decrement $\delta(\mathbf{r})$ can be directly linked to the electron density $\rho_{e}(\mathbf{r})$ by $[3]$

$$
\delta(\mathbf{r}) \simeq \frac{2 \pi r_{e}}{k^{2}} \rho_{e}(\mathbf{r}),
$$

with the Thomson scattering length $r_{e} \simeq 2.818 \cdot 10^{-15} \mathrm{~m}$. As can be seen in Fig. 1.3, no absorption edges are present in the energy range between 7 and $20 \mathrm{keV}$, hence Eq. (1.50) will give a good approximation. For low-Z elements other than hydrogen, one can also find an approximate relation between $\delta(\mathbf{r})$ and the mass 

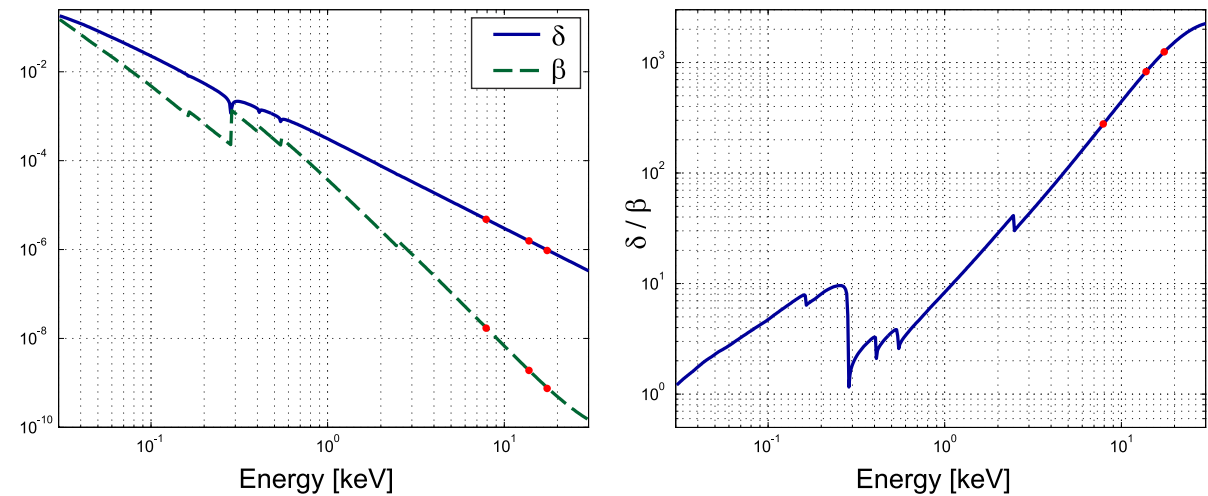

Figure 1.3: The refractive index (left) and the ratio $\delta / \beta$ (right) for a model protein with chemical formula $H_{50} C_{30} N_{9} O_{10} S_{1}[78]$ and mass density $1.35 \mathrm{~g} / \mathrm{cm}^{3}$. Three typical energies used in this thesis at $7.9 \mathrm{keV}, 13.8 \mathrm{keV}$ and $17.5 \mathrm{keV}$ are indicated with red dots. Values are obtained from [71].

density $\rho_{m}(\mathbf{r})$, i.e., [53]

$$
\delta(\mathbf{r}) \simeq \frac{\pi r_{e}}{k^{2} u} \rho_{m}(\mathbf{r})
$$

with the atomic mass unit $u \simeq 1.661 \cdot 10^{-27} \mathrm{~kg}$. Note that although hydrogen is present in the generic biological material, its mass percentage is very low. Hence, Eq. (1.51) will still be a good approximation in this case and the reconstructed phase can be mapped to an effective mass density [53]. For the imaginary part $\beta$ of the refractive index, a similar relationship cannot be found as easily. Consequently, the reconstructed values can be used as a measure for quantitative mass distributions only if the interaction via $\delta(\mathbf{r})$ is exploited [183]. However, for single material elements and away from absorption edges, $\beta \propto Z^{4} E^{-4}$ applies [3].

\subsubsection{Projection approximation}

The interaction between x-rays and matter can be expressed as absorption (i.e. the intensity behind the sample will be less than before) or as diffraction (i.e. radiation will change its propagation direction). There are different mechanisms on the atomic level like photoelectric absorption, (in)elastic scattering, x-ray fluorescence or Auger scattering, which can lead to a complicated behavior [3]. In the following, we consider the case of a weakly interacting, thin specimen that is placed in a paraxial beam. Starting with the inhomogeneous Helmholtz equation (1.45) and using the same separation ansatz and derivation as in the free-space case, one 
arrives at the inhomogeneous paraxial wave equation [133]

$$
\left(\nabla_{\perp}^{2}+2 \mathrm{i} k \partial_{z}+k^{2}\left(n(\mathbf{r})^{2}-1\right)\right) \psi^{\prime}(\mathbf{r})=0 .
$$

As $\delta(\mathbf{r})$ and $\beta(\mathbf{r})$ in the refractive index are generally much smaller than unity, we omit quadratic terms in $n^{2}(\mathbf{r})$, thus it is

$$
n^{2}(\mathbf{r})=1-2 \delta+2 \mathrm{i} \beta
$$

The projection approximation manifests itself in the assumption that scattering effects inside the specimen can be neglected, i.e., phase shift and absorption are completely determined by considering interactions with infinitesimal thin samples. For Eq. (1.52) this means that the effect of the lateral Laplacian $\nabla_{\perp}^{2}$ will be much smaller than that of the axial derivative $\partial_{z}$. Inside the sample the phase shift and absorption will be cumulated but will have no effect on the propagation of the wave. Behind the sample the disturbed wave propagates in free-space again, as illustrated in Fig. 1.4. This approximation corresponds to the classical limiting case of geometrical optics [133]. A criterion for the validity is e.g., given by [7]

$$
\sqrt{\frac{\lambda d}{2}}<\Delta r_{\perp}
$$

in which $\Delta \mathbf{r}_{\perp}$ corresponds to the smallest structure present. As biological specimens vary on all length scales, this will be typically the smallest resolution element of the imaging system. An equivalent formulation of Eq. (1.54) is given by

$$
d<\frac{2 \Delta r_{\perp}^{2}}{\lambda},
$$

thus defining an upper limit for the sample thickness. Assuming $\lambda=1 \AA=$ $0.1 \mathrm{~nm}$ and $\Delta r_{\perp}=1 \mu \mathrm{m}$, the maximum thickness according to Eq. (1.55) will be $d=20 \mathrm{~mm}$. This criterion is not strict and prefactors vary in the literature. To avoid artifacts due to thicker specimens, multi-slice approaches have been used successfully [167].

After applying the discussed approximation, Eq. (1.52) will become the very simple differential equation

$$
\partial_{z} \psi^{\prime}(\mathbf{r})=-\mathrm{i} k \Delta n(\mathbf{r}) \psi^{\prime}(\mathbf{r}),
$$

in which $\Delta n=1-n=\delta-\mathrm{i} \beta$ is introduced. Integrating the above equation for a 


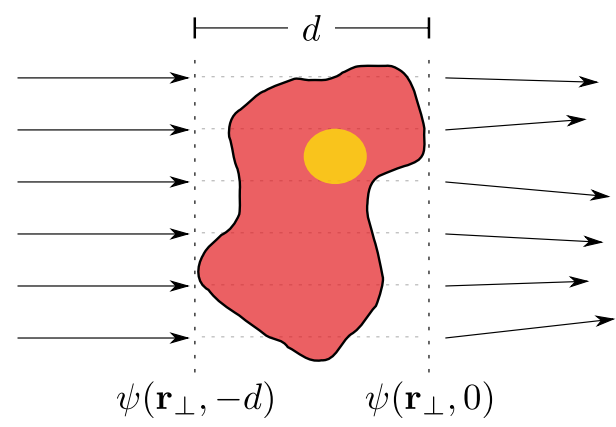

Figure 1.4: Illustration of the projection approximation: An incoming wave field $\psi\left(\mathbf{r}_{\perp},-d\right)$ will be changed by the specimen, such that absorption and phase shift will be cumulated in an exit plane $\psi\left(\mathbf{r}_{\perp}, 0\right)$ without accounting for internal scattering. Thus, for a pure phase object the intensity directly behind the object will be the same as directly before the object and will transform into measurable changes after a free-space propagation.

given illumination $\psi^{\prime}\left(\mathbf{r}_{\perp},-d\right)=\psi_{0}^{\prime}$ yields [133]

$$
\psi^{\prime}\left(\mathbf{r}_{\perp}, 0\right)=\psi_{0}^{\prime} \cdot \mathbf{e}^{-\mathbf{i} k \int_{-d}^{0} \Delta n(\mathbf{r}) \mathbf{d} z} .
$$

We introduce $\bar{\delta}\left(\mathbf{r}_{\perp}\right)=\int_{-d}^{0} \delta(\mathbf{r}) \mathrm{d} z$ and $\bar{\beta}\left(\mathbf{r}_{\perp}\right)=\int_{-d}^{0} \beta(\mathbf{r}) \mathrm{d} z$, which describe the specimen by an effective refractive index in the exit plane. Substituting Eq. (1.57) in the ansatz of a paraxial wave gives an expression for the so-called exit wave

$$
\psi\left(\mathbf{r}_{\perp}, 0\right)=\psi_{0} \cdot \mathrm{e}^{\mathrm{i} k d} \cdot \mathrm{e}^{-\mathrm{i} k \bar{\delta}\left(\mathbf{r}_{\perp}\right)} \cdot \mathrm{e}^{-k \bar{\beta}\left(\mathbf{r}_{\perp}\right)}=: \mathrm{e}^{\mathrm{i} k d} \cdot P\left(\mathbf{r}_{\perp}\right) \cdot O\left(\mathbf{r}_{\perp}\right)
$$

This result is typically written as a product of the probe function

$$
P\left(\mathbf{r}_{\perp}\right):=\psi^{\prime}\left(\mathbf{r}_{\perp},-d\right)=\psi_{0},
$$

and the object transmission function

$$
O\left(\mathbf{r}_{\perp}\right):=\mathrm{e}^{-\mathrm{i} k \bar{\delta}\left(\mathbf{r}_{\perp}\right)} \cdot \mathrm{e}^{-k \bar{\beta}\left(\mathbf{r}_{\perp}\right)} .
$$


We further define the phase $\phi(\mathbf{r})$ and the absorption $\mu(\mathbf{r})$ of the object

$$
\begin{aligned}
& \phi\left(\mathbf{r}_{\perp}\right)=-k \int_{-d}^{0} \delta(\mathbf{r}) \mathrm{d} z \\
& \mu\left(\mathbf{r}_{\perp}\right)=2 k \int_{-d}^{0} \beta(\mathbf{r}) \mathrm{d} z
\end{aligned}
$$

The influence of the specimen can be described by Eq. (1.60). In an imaging experiment, the wave field directly behind the sample can be reconstructed by suitable phase-retrieval algorithms. Further, if the probe $P\left(\mathbf{r}_{\perp}\right)$ is also reconstructed or known a priori, one can directly obtain the projected refractive index using Eq. (1.60). With the assumption of a normalized plane wave illumination $\mathrm{e}^{\mathrm{i} k z}$, the exit wave directly corresponds to the object transmission function and thus the phase and amplitude of the exit wave yield $\phi\left(\mathbf{r}_{\perp}\right)$ and $\mu\left(\mathbf{r}_{\perp}\right)$. In combination with tomography, described in chapter 3 , the projected quantities can be used to reconstruct the spatial distribution of $\delta(\mathbf{r})$ and $\beta(\mathbf{r})$. We remind the reader that $\mu(\mathbf{r})$ is not to be confused with the linear attenuation coefficient

$$
\mu_{L B}=2 k \beta
$$

often used in classical tomography as a result of the Lambert-Beer law. It differs from $\mu(\mathbf{r})$ here defined by the missing dependence on the thickness along the optical $\operatorname{axis} z$.

\subsection{From wave fields to images}

So far we have derived tools to describe the free-space propagation of wave fields and the interaction of x-rays with matter. Let us again consider the situation sketched in Fig. 1.4: We have an illuminating plane wave, indicated by parallel arrows, which interacts with the sample and thereby changes its phase and amplitude distribution in the exit plane. For a pure phase object, the intensity of the wave, indicated by the line density, does not change and, measured directly behind the object at $z=0$, it will still look like the illuminating plane wave. However, the phase distribution of the wave also has changed, which leads to spatially-dependent distribution of propagation directions. After a finite propagation distance $z$, the wave will interfere with itself and thereby change the intensity. This intensity distribution can be measured. Hence, we will be able to see structures of the object, although they did not absorb any radiation. The following chapter will treat this 
effect in a mathematical way, allowing the quantitative description and providing the basis for phase-retrieval algorithms in chapter 2 .

\subsubsection{Contrast transfer function}

We want to derive an expression for the intensity $I(\mathbf{r})=|\psi(\mathbf{r})|^{2}$ behind an object. Let the illumination be a normalized plane wave $P\left(\mathbf{r}_{\perp}\right)=\mathrm{e}^{\mathrm{i} k \cdot 0}=1$. Hence, the exit wave is determined by the object transmission function (1.60) and a constant phase factor, where the latter will have no influence on the intensity. Considering the case of a weak object, i.e., $\phi(\mathbf{r}) \ll 1$ and $\mu(\mathbf{r}) \ll 1$, Eq. (1.60) can be linearized, yielding

$$
O\left(\mathbf{r}_{\perp}\right)=1-\mu\left(\mathbf{r}_{\perp}\right) / 2+i \phi\left(\mathbf{r}_{\perp}\right) .
$$

To calculate the propagated wave field in a distance $z$ to the exit plane, we use the Fresnel diffraction operator (1.24)

$$
\begin{aligned}
\psi\left(\mathbf{r}_{\perp}, z\right) & =\mathcal{D}_{z}\left(\psi\left(\mathbf{r}_{\perp}, 0\right)\right) \\
& =\mathcal{D}_{z}\left(\mathrm{e}^{\mathrm{i} k d} \cdot O\left(\mathbf{r}_{\perp}\right)\right) \\
& =\mathrm{e}^{\mathrm{i} k d} \mathcal{F}_{\perp}^{-1}\left[\mathrm{e}^{-\mathrm{i} \chi} \mathcal{F}_{\perp}\left(1-\mu\left(\mathbf{r}_{\perp}\right) / 2+\mathrm{i} \phi\left(\mathbf{r}_{\perp}\right)\right)\right]
\end{aligned}
$$

Here, we introduced the abbreviation

$$
\chi=\frac{z}{2 k}\left(k_{x}^{2}+k_{y}^{2}\right)=\pi \lambda z\left(\nu_{x}^{2}+\nu_{y}^{2}\right)
$$

where we also note the expression in dependence on the spatial frequencies $\nu_{x / y}=$ $k_{x / y} / 2 \pi$, often used to define the Fourier transform without prefactors [55]. If pure phase terms are neglected and the relation $\mathrm{e}^{-\mathrm{i} \chi}=\cos \chi-\mathrm{i} \sin \chi$ is utilized, Eq. (1.65) can be written as

$$
\psi\left(\mathbf{r}_{\perp}, z\right)=1+\mathcal{F}_{\perp}^{-1}\left[(\cos \chi-\mathrm{i} \sin \chi)\left(\mathrm{i} \tilde{\phi}\left(\mathbf{k}_{\perp}\right)-\tilde{\mu}\left(\mathbf{k}_{\perp}\right) / 2\right)\right]
$$

where $\tilde{\phi}\left(\mathbf{k}_{\perp}\right)$ and $\tilde{\mu}\left(\mathbf{k}_{\perp}\right)$ denote the lateral Fourier transforms of $\phi\left(\mathbf{r}_{\perp}\right)$ and $\mu\left(\mathbf{r}_{\perp}\right)$. A multiplication in Fourier space can be expressed as a convolution in real space, so an equivalent formulation of this equation is

$$
\begin{aligned}
\psi\left(\mathbf{r}_{\perp}, z\right)= & 1+\mathcal{F}_{\perp}^{-1}[\mathrm{i} \tilde{\phi} \cos \chi+\tilde{\phi} \sin \chi-\tilde{\mu} / 2 \cos \chi+\mathrm{i} \tilde{\mu} / 2 \sin \chi] \\
= & 1+\mathrm{i} \phi * \mathcal{F}_{\perp}^{-1}(\cos \chi)+\phi * \mathcal{F}_{\perp}^{-1}(\sin \chi) \\
& -\mu / 2 * \mathcal{F}_{\perp}^{-1}(\cos \chi)+\mathrm{i} \mu / 2 * \mathcal{F}_{\perp}^{-1}(\sin \chi)
\end{aligned}
$$




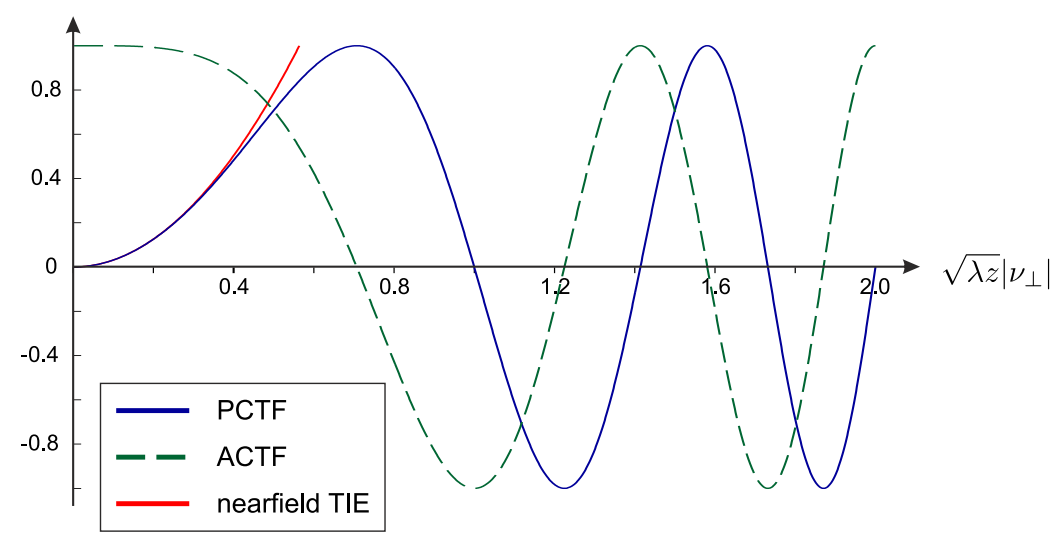

Figure 1.5: Contrast transfer functions in dependence on a reduced spatial frequency. The low-frequency part is well approximated by quadratic increase of the near-field TIE. Note the zero crossings, meaning that for these spatial frequencies no information about phase or amplitude is transferred.

where $*$ denotes the convolution. With this equation, we are able to calculate the intensity by

$$
\begin{aligned}
I\left(\mathbf{r}_{\perp}, z\right) & =\left|\psi\left(\mathbf{r}_{\perp}, z\right)\right|^{2} \\
& \approx 1+2 \phi * \mathcal{F}_{\perp}^{-1}(\sin \chi)-\mu * \mathcal{F}_{\perp}^{-1}(\cos \chi)
\end{aligned}
$$

Fourier transformation of Eq. (1.69) yields the intensity in reciprocal space

$$
\tilde{I}\left(\mathbf{k}_{\perp}, z\right)=2 \pi \delta_{D}\left(\mathbf{k}_{\perp}\right)+2 \tilde{\phi}\left(\mathbf{k}_{\perp}\right) \sin \chi-\tilde{\mu}\left(\mathbf{k}_{\perp}\right) \cos \chi
$$

where $\delta_{D}$ is the Dirac delta distribution. An equivalent formulation, often found in the literature, is obtained by considering $\boldsymbol{\nu}_{\perp}=\left(\nu_{x}, \nu_{y}\right)^{T}$ instead of $\mathbf{k}_{\perp}=\boldsymbol{\nu}_{\perp} \cdot 2 \pi$

$$
\tilde{I}\left(\boldsymbol{\nu}_{\perp}, z\right)=\delta_{D}\left(\boldsymbol{\nu}_{\perp}\right)+2 \tilde{\phi}\left(\boldsymbol{\nu}_{\perp}\right) \sin \chi-\tilde{\mu}\left(\boldsymbol{\nu}_{\perp}\right) \cos \chi
$$

which is known as the contrast transfer function (CTF) [140, 59]. In the latter case, $\chi$ has to depend on $\boldsymbol{\nu}_{\perp}$ as described in Eq. (1.66).

Equation (1.71) can be interpreted as follows: For vanishing propagation distance, i.e., $\chi=0$, the Fourier transform of the intensity corresponds to the directly transmitted radiation represented by the delta distribution and the unfiltered absorption $\tilde{\mu}$. In particular, for a pure phase object with $\mu=0$ this means that the intensity 
in the exit plane will be just the plane-wave illumination. Phase and absorption of the object act as additive perturbations, filtered with $\sin \chi$ and $\cos \chi$ for $\phi$ and $\mu$, respectively. Figure 1.5 illustrates these filter kernels in the Fourier domain for a reduced spatial frequency $\sqrt{\lambda z}\left|\boldsymbol{\nu}_{\perp}\right|$ that are termed phase contrast-transfer function (PCTF) and amplitude contrast-transfer function (ACTF). For increasing propagation distances, the phase map $\phi$ will influence the intensity distribution more and the overall contrast transfer will become stronger. Due to the oscillatory type of the CTFs, some spatial frequencies will not contribute to the intensity, as $\sin \chi$ or $\cos \chi$ can vanish. These findings were experimentally validated in [153]. It also has to be mentioned that the CTF (1.71) can be derived in a different way, starting with a representation of the propagation as a convolution rather than a multiplication in Fourier space as used here. With this alternative derivation it was shown that for homogenous objects the assumption of a weak object can be relaxed to a weak absorption and slowly varying phase [172].

Next, we want to briefly consider the special case of a pure phase object, i.e., $\mu=0$, and small propagation distances, i.e., $\sin \chi \approx \chi$. Equation (1.70) then reads

$$
\tilde{I}\left(\mathbf{k}_{\perp}, z\right) \simeq 2 \pi \delta_{D}\left(\mathbf{k}_{\perp}\right)+2 \tilde{\phi}\left(\mathbf{k}_{\perp}\right) \chi
$$

Substituting the definition of $\chi$ given by Eq. (1.66) and performing the Fourier back transform yields

$$
I\left(\mathbf{r}_{\perp}, z\right) \simeq 1+\frac{z}{k} \nabla_{\perp}^{2} \phi\left(\mathbf{r}_{\perp}\right)
$$

Before we discuss the meanings of this equation, we will consider a different derivation in the next section.

\subsubsection{Transport of intensity equation}

An alternative differential equation to describe the behavior of electromagnetic waves in free space can be derived from the paraxial wave equation in vacuum (1.16) with the ansatz

$$
\psi^{\prime}(\mathbf{r})=\sqrt{I(\mathbf{r})} \mathrm{e}^{\mathrm{i} \phi(\mathbf{r})},
$$

where a complex solution to Eq. (1.16) is described by the measurable intensity $I(\mathbf{r})=|\psi(\mathbf{r})|^{2}$ and the phase distribution $\phi(\mathbf{r})$, both being real-valued fields. If an object is placed at $z \in[-d, 0]$ as before, the phase $\phi\left(\mathbf{r}_{\perp}, 0\right)$ will correspond to the object phase defined in Eq. (1.61), but in general $\phi(\mathbf{r})$ introduced here, additionally depends on the axial position $z$. After inserting the ansatz (1.74) in the paraxial wave equation (1.16) and performing some simplifications, the imaginary part can 
be identified as the so-called transport of intensity equation (TIE) [64, 166]

$$
\nabla_{\perp} \cdot\left(I(\mathbf{r}) \nabla_{\perp} \phi(\mathbf{r})\right)=-k \frac{\partial I(\mathbf{r})}{\partial z}
$$

This continuity equation states that for known intensity $I(\mathbf{r})$ and phase $\phi(\mathbf{r})$ the evolution of a wave along the optical axis can be calculated for a small step $\partial z$. It also can be used for several phase-retrieval algorithms as explained in more detail in chapter 2. For a pure phase object, the intensity directly behind the object corresponds to the illumination that we again assume to be a plane wave with intensity $I_{0}$, i.e., $I\left(\mathbf{r}_{\perp}, 0\right)=I_{0}=$ const. If we further consider a small propagation distance $\Delta z$, the differential quotient in Eq (1.75) can be approximated by the forward difference quotient

$$
\nabla_{\perp}^{2} \phi\left(\mathbf{r}_{\perp}, 0\right) \simeq-k \frac{I\left(\mathbf{r}_{\perp}, \Delta z\right)-I_{0}}{I_{0} \Delta z}
$$

This equation can be solved for $I\left(\mathbf{r}_{\perp}, z\right)$, yielding the same expression ${ }^{5}$ as derived from the CTF before

$$
\frac{I\left(\mathbf{r}_{\perp}, \Delta z\right)}{I_{0}} \simeq 1+\frac{\Delta z}{k} \nabla_{\perp}^{2} \phi\left(\mathbf{r}_{\perp}, 0\right) .
$$

Regarding the left-hand side of Eq. (1.75), i.e.

$$
\nabla_{\perp} \cdot\left(I(\mathbf{r}) \nabla_{\perp} \phi\left(\mathbf{r}_{\perp}, 0\right)\right)=I(\mathbf{r}) \nabla_{\perp}^{2} \phi\left(\mathbf{r}_{\perp}, 0\right)+\underbrace{\nabla_{\perp} I(\mathbf{r}) \cdot \nabla_{\perp} \phi\left(\mathbf{r}_{\perp}, 0\right)}_{!}
$$

the second summand has to disappear in order to obtain the structure of Eq. $(1.73)$ or $(1.77)$. For a pure phase object this is generally true. However, even if $\nabla_{\perp} I(\mathbf{r})$ is not small enough (e.g. due to a finite absorption) the above derivation still holds, if the product of $\nabla_{\perp} I(\mathbf{r})$ and $\nabla_{\perp} \phi(\mathbf{r})$ is small enough. This will for example be the case if a slowly varying phase is considered.

Equation (1.77), which we will denote the nearfield TIE, states that the intensity distribution of a pure phase object for small propagation distances corresponds to the Laplacian of the phase distribution. In a physical interpretation this means that edges of an image will be enhanced. We will denote this very important special case as the direct-contrast regime. Importantly, as the dependence on

5 In the derivation of the CTF we assumed the illumination to be a normalized plane wave with $I_{0}=1$. 
$\lambda$ in Eq. (1.77) is just a scaling factor, the equation also holds for a broader range of photon energies and partially coherent illumination $[132,185]$, which makes it very important for the use at laboratory sources.

\subsubsection{Imaging regimes}

The effect of free-space propagation can to some extent be understood with the CTF. However, a full understanding of how an image will appear is hard to obtain from the simplified formulas. To illustrate the effect of free-space propagation, Fig. 1.6 shows simulated intensity distributions for several propagation distances. The object used for the simulation consists of parts that only absorb the radiation and induce no phase shift (the A's), of objects that only change the phase and do not absorb any radiation (the $\mathrm{P}$ 's and the eye) and of mixed parts that do both (the M's and the circle). As the paraxial propagation solely depends on the Fresnel number $F=a^{2} / \lambda z$, it can be used as a measure for the kind of diffraction that occurs. Here we define a typical feature to have a size of ten pixels. We will note the number of pixels, used to define the Fresnel number, with a superscript at the Fresnel number

$$
F^{10}=\frac{(10 \cdot p)^{2}}{\lambda z}=100 \cdot F^{1}
$$

For the simulation a numerical implementation of Eq. (1.24) is used. The images have a size of $N_{x}=N_{y}=606$ quadratic Pixels $\left(p=p_{x}=p_{y}\right)$ and the Fresnel numbers are chosen to be in the range of $F^{10} \in[0.002,10]$. For a pixel size of $p=0.1 \mu \mathrm{m}$ and a wavelength of $\lambda=1 \AA$ this corresponds to propagation distances between $z=1 \mathrm{~mm}$ and $z=5 \mathrm{~m}$.

For $F \gg 1$, hence very small propagation distances, and $\sin \chi \approx 0$, no diffraction effects can be observed and the image will only contain unfiltered information about absorbing structures since $\cos \chi \approx 1$. This regime is called contact regime or the regime of geometric optics, being well described by the classical ray picture widely used, e.g., for absorption imaging in classical radiology. As the propagation distance increases, Fresnel diffraction will start to occur but the image still shows resemblance to the original image. For $F^{10} \approx 10$ the before-mentioned directcontrast regime is present, in which phase shifting structures become visible (cf. Fig. 1.6(b)). Here, the image formation is well described by the nearfield TIE (1.77), thus the curvature of the phase distribution dominates the image. As edges exhibit strongest curvature, diffraction is reduced to edge enhancement and 

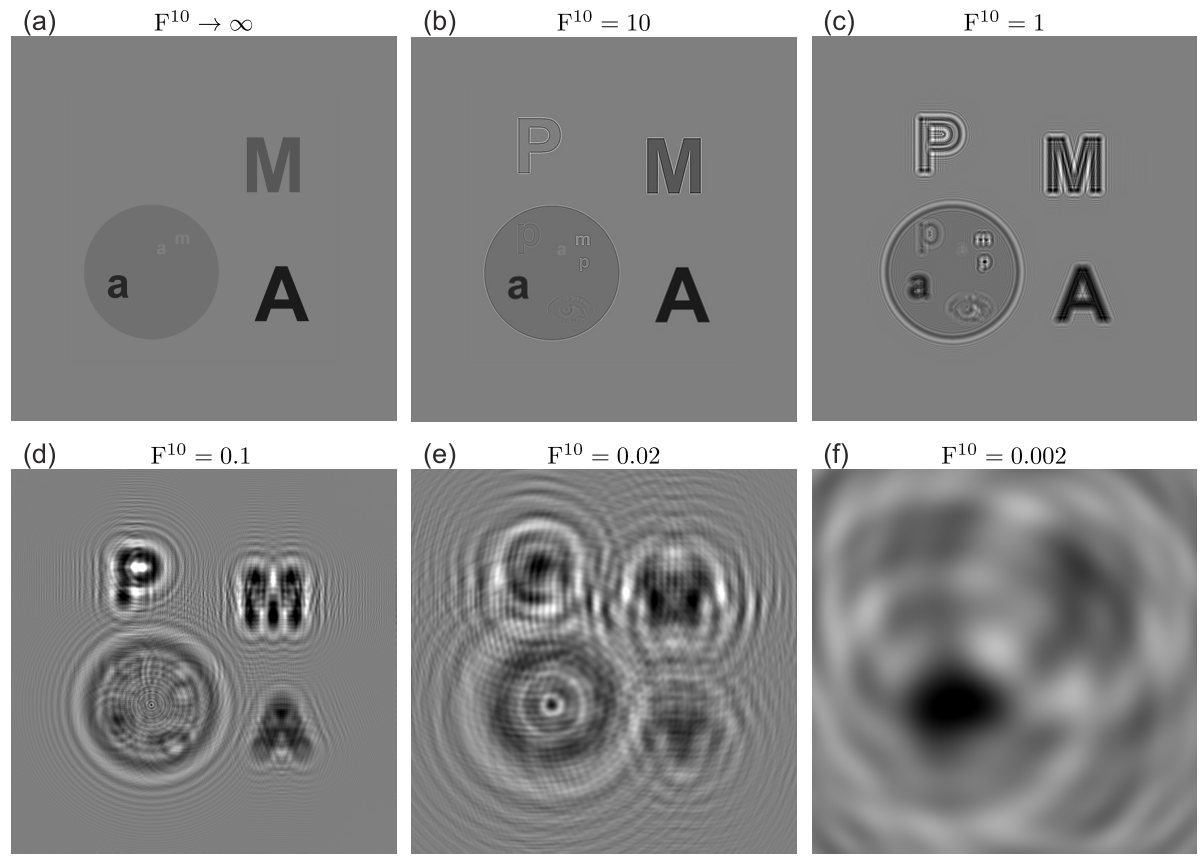

Figure 1.6: Simulated intensity distributions for different Fresnel numbers $F^{10}$ showing the effect of free-space propagation under coherent illumination. The object consists of purely absorbing objects (A), mixed objects ( $M$ and the circle) and pure phase objects ( $P$ and the eye) with different strength. The minimal transmission is chosen to be 0.76 and the maximum phase shift to $0.55 \mathrm{rad}$. The gray scale for all images ranges from 0.5 (black) to 1.5 (white).

the original image can still be recognized quite well. ${ }^{6}$ However, little information about low spatial frequencies is yet contained in the image, as the PCTF increases only slowly. Especially structures embedded in a surrounding (e.g. the eye in the simulation or biological tissues embedded in water) are barely visible and to optimize contrast transfer, smaller Fresnel numbers are beneficial. For $F^{10} \lesssim 1$ the measurable intensity distribution loses resemblance to the original object and multiple oscillatory variations, so-called fringes, can be observed. In this (near) holographic regime, low-frequency structures such as the large letters, can still be recognized without further treatment of the image. This can be explained, by considering the Fresnel number for a larger structure that will still indicate the

${ }^{6}$ Note that "white fringes", i.e., regions where the intensity is larger than in the illumination can be used to proof the presence of phase contrast, as they are effects of constructive interference. 
direct-contrast regime. For even smaller $F^{10}$, one speaks of the image becoming more and more "holographic", in correspondence to the original idea of holography introduced by Gabor already in 1948 [51]. This region is also-called the deep holographic regime, in which the contrast transfer for all spatial frequencies is high. However, the direct interpretation of such an image is difficult (cf. Fig. 1.6(e)). By the use of phase-retrieval algorithms, the original structure can be obtained from a measured hologram, as described in detail in chapter 2. Imaging techniques using a coherent illumination, which allows the observation of propagation effects described here, are referred to as propagation-based phase-contrast imaging, in-line holography or propagation imaging. After its original proposal for electron beams [51], applications with x-ray radiation were first demonstrated at synchrotron storage rings at the end of the last century [36, 123, 125].

For $F \ll 1$ the Fraunhofer regime will be reached, being well described by the Fourier transform. Note that the Fresnel number for the largest structure should be much smaller than one. In the example given in Fig. 1.6(f) the Fresnel number for the whole image is $F^{600}=7.2$, thus the Fraunhofer regime is not reached yet. Imaging techniques operating in this regime are termed coherent diffractive imaging (CDI) and are more demanding in terms of spatial coherence, as every point in the imaging plane interferes with every other point. They were first introduced as a generalization of x-ray crystallography [109] and, iterative phaseretrieval algorithms are commonly used to obtain an impression of the original structure [46]. One particular method, using a scanning approach with coherent illumination is known as Ptychography [147, 148]. Here scan points are recorded so that a partial overlap to other points is present, which is used as a constraint to reconstruct the wave field in the object exit-plane.

\subsubsection{Fresnel scaling theorem}

In many cases $\mathrm{x}$-ray imaging is performed in a divergent beam geometry that can be obtained, e.g., by illumination of an object with a spherical wave. Its analytical treatment is quite complicated, but as we will show in this section, the introduction of an effective parallel beam geometry simplifies the cone-beam case. This will allow treating propagation experiments with cone-beam illumination as a parallel beam experiment, as long as a geometric transform is used.

Let us consider a spherical wave, with its origin on the optical axis at a distance $z_{1}$ before an object, the exit plane of which is chosen to be at $z=0$ (cf. Fig. 1.7). If we assume the distance between sample and origin to be large enough, we can 

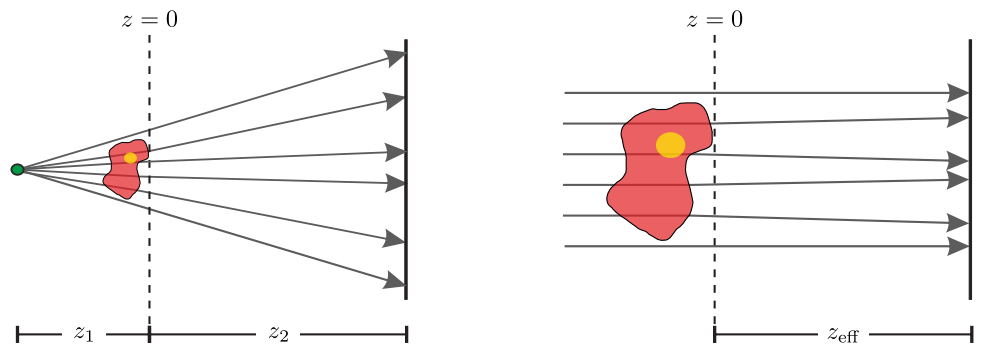

Figure 1.7: Illustration of the Fresnel scaling theorem: A point source at a distance $z=-z_{1}$ illuminates an object at $z=0$, creating a divergent beam that will magnify the image of the object on a detector at distance $z=z_{2}$. The same situation can be described in an effective parallel beam geometry, in which a wave, sampled with effectively smaller pixels, propagates an effective distance $z_{\text {eff. }}$

describe the illumination in paraxial approximation by Eq. (1.17) and with the projection approximation, the wave function in the exit plane reads

$$
\psi\left(\mathbf{r}_{\perp}, 0\right)=\frac{1}{z_{1}} \cdot \mathrm{e}^{\frac{\mathrm{i} k}{2 z_{1}} \mathbf{r}_{\perp}^{2}} \cdot O\left(\mathbf{r}_{\perp}\right)
$$

where $O\left(\mathbf{r}_{\perp}\right)$ denotes the object transmission function. The propagated field at distance $z=z_{2}$ can then be described, e.g., by the Huygens-Fresnel principle given by Eq. (1.32). Substituting the ansatz (1.80) into this integral yields

$$
\psi\left(\mathbf{r}_{\perp}, z_{2}\right)=\frac{C\left(z_{2}\right)}{z_{1}} \int_{\mathbb{R}^{2}} O\left(\mathbf{r}_{\perp}\right) \mathrm{e}^{\frac{\mathrm{i} k}{2} r_{\perp, 0}^{2}\left(\frac{1}{z_{1}}+\frac{1}{z_{2}}\right)} \mathrm{e}^{-\frac{\mathrm{i} k}{z_{2}} \mathbf{r}_{\perp, 0} \cdot \mathbf{r}_{\perp}} \mathrm{d} \mathbf{r}_{\perp, 0},
$$

where $C\left(z_{2}\right)=\frac{\mathrm{e}^{\mathrm{i} k z_{2}}}{\mathrm{i} \lambda z_{2}} \mathrm{e}^{\frac{\mathrm{i} k}{2 z_{2}} r_{\perp}^{2}}$ is defined as for Eq. (1.32). Equation (1.81) motivates the definition of an effective propagation distance

$$
\frac{1}{z_{\mathrm{eff}}}:=\frac{1}{z_{1}}+\frac{1}{z_{2}}=\frac{M}{z_{2}},
$$

and the geometric magnification

$$
M=\frac{z_{1}+z_{2}}{z_{1}}
$$


Hence, it is $z_{2}=z_{\text {eff }} \cdot M$ and Eq. (1.81) reads

$$
\psi\left(\mathbf{r}_{\perp}, z_{2}\right)=\frac{C\left(z_{2}\right)}{z_{1}} \int_{\mathbb{R}^{2}} O\left(\mathbf{r}_{\perp}\right) \mathrm{e}^{\frac{\mathrm{i} k}{2 z_{\mathrm{eff}}} r_{\perp, 0}^{2}} \mathrm{e}^{-\frac{\mathrm{i} k}{z_{\mathrm{eff}}} \frac{\mathbf{r}_{\perp, 0}}{M} \cdot \mathbf{r}_{\perp}} \mathrm{d} \mathbf{r}_{\perp, 0}
$$

Next, we consider the object under plane wave illumination, where we normalize the illumination to be $\left|P\left(\mathbf{r}_{\perp}\right)\right|=1 / z_{1}$. Thus, the illumination has the same intensity as the spherical wave in the object plane. The wave field after propagation over the distance $z_{\text {eff }}$ is again given by Eq. (1.32)

$$
\psi^{(\mathrm{P})}\left(\mathbf{r}_{\perp}, z_{2}\right)=\frac{C\left(z_{\mathrm{eff}}\right)}{z_{1}} \int_{\mathbb{R}^{2}} O\left(\mathbf{r}_{\perp}\right) \mathrm{e}^{\frac{\mathrm{i} k}{2 z_{\mathrm{eff}}} r_{\perp, 0}^{2}} \mathrm{e}^{-\frac{\mathrm{i} k}{z_{\mathrm{eff}}} \mathbf{r}_{\perp, 0} \cdot \mathbf{r}_{\perp}} \mathrm{d} \mathbf{r}_{\perp, 0}
$$

To compare the intensity distribution $I\left(\mathbf{r}_{\perp}, z_{2}\right)=\left|\psi\left(\mathbf{r}_{\perp}, z_{2}\right)\right|^{2}$, we note that

$$
\left|C\left(z_{2}\right)\right|=\left|C\left(z_{\mathrm{eff}} \cdot M\right)\right|=1 / M\left|C\left(z_{\mathrm{eff}}\right)\right| .
$$

Thus, we find that

$$
I\left(\mathbf{r}_{\perp}, z_{2}\right)=\frac{1}{M^{2}} I^{(\mathrm{P})}\left(\frac{\mathbf{r}_{\perp}}{M}, z_{2}\right)
$$

known as the Fresnel scaling theorem [133]. Hence, the propagation of an exit wave, created by illumination with a parabolic wave can be written as a planewave propagation with effective distance $z_{e f f}$, where the lateral distances in the object plane will be demagnified by the geometric magnification $M$. Note that the $1 / M^{2}$ scaling of the intensities in Eq. (1.87) accounts for energy conservation upon propagation [133].

Let us briefly regard some experimentally relevant parameters and their implications to the imaging regime: Assume a detector with quadratic pixels of $p=5 \mu \mathrm{m}$ size that is placed at $z_{02}=z_{1}+z_{2}=5 \mathrm{~m}$ behind an effective secondary source. A sample is located at $z_{1}=50 \mathrm{~mm}$, leading to a geometric magnification of $M=100$ and thus an effective pixel size of $p_{\text {eff }}=50 \mathrm{~nm}$ and an effective propagation distance $z_{\text {eff }}=49.5 \mathrm{~mm}$. The resulting Fresnel number for a wavelength of $\lambda=1 \AA$ is

$$
F^{10}=\frac{\left(10 \cdot p_{\text {eff }}\right)^{2}}{\lambda z_{\text {eff }}}=\frac{100 \cdot p^{2}}{\lambda z_{2} \cdot M}=0.202 .
$$

For $z_{1} \ll z_{2}$ and a fixed detector distance $z_{02}$, the distance $z_{2}=z_{02}-z_{1}$ is nearly constant, and thus the Fresnel number scales with the geometric magnification. Upon reducing $z_{1}$, images will be in the near holographic regime and for decreasing $z_{1}$ the transition to the deep holographic regime will occur.

A part of the experiments is performed at synchrotron storage rings, where a 
secondary source is created by focusing x-rays. Here, in principle one could place objects not only far away from the focus but also in the focus. For the assumption of a Gaussian beam, it was shown that the maximum magnification is limited and that a scaling law similar to the Fresnel scaling theorem can be found [182]. In a perfect focal plane, the phase distribution is a constant. Thus, no magnification will occur and the propagation over a distance of $5 \mathrm{~m}$ will lead to a measurement in the Fraunhofer regime.

\subsection{Coherence}

So far we have assumed perfectly coherent beams. One way to think of a fully coherent beam is to assume a monochromatic plane wave. A real beam deviates from this assumption in two ways: It can be temporally incoherent, i.e., the beam will not be monochromatic or it can be spatially incoherent, i.e., its propagation directions can deviate from the assumed direction [3]. While the temporal coherence of a wave can be tuned by using monochromators, e.g., Bragg reflections in a single crystal, the spatial coherence can be tuned by the experiment geometry. To better understand the concept of spatial coherence, an analogy is given by the following gedankenexperiment: Imagine two people Alice and Bob, separated by some distance $z$. Alice has two flashlights in her hands with a fixed distance to each other that shine in the direction of Bob. For small $z$, Bob is close to Alice and he sees the light of both flashlights separated with different propagation directions. If Bob is far away from Alice and thus $z$ is larger, at some point he will not be able to distinguish both lights and the light seems to come from one point, with only a single propagation direction. In the first case the spatial coherence is very low as the observer can see the different propagation directions. In the second case, the spatial coherence of the light has increased just by increasing the distance $z$ between interaction point (where a sample would be placed) and source. The spatial coherence of an x-ray beam can be increased similarly by using, e.g., a small source size and/or large distance to the source. X-ray waveguides, which are of particular relevance for the present work, are also able to increase the spatial coherence by mode filtering [127], i.e., only coherent modes survive the propagation in a wave guiding channel.

For this work, we need to keep in mind that partial incoherence can limit the maximum resolution in an experiment. However, the imaging regime has a considerable influence. Especially for propagation-based phase-contrast, a fully coherent illumination is not necessary [140]. For an image becoming more holographic, a higher 
degree of coherence is required to enable observation of fine interference fringes, responsible for high resolution information. In particular, for a given temporal coherence, described by the wavelength spread $\Delta \lambda$, effects of partial coherence can be neglected if $[35,153]$

$$
\Delta \chi=\pi z \nu^{2} \Delta \lambda \ll \pi,
$$

for all spatial frequencies $\nu$. Equation (1.89) can be rearranged to yield an equivalent expression that depends on the Fresnel number $F$ and on the relative bandwidth $\Delta \lambda / \lambda$

$$
\frac{\Delta \lambda}{\lambda} \frac{\lambda z}{4 a^{2}}=\frac{\Delta \lambda}{\lambda} \frac{1}{4 F} \ll 1,
$$

with the typical feature size $a$ that in most cases will be given by the smallest resolvable resolution element, e.g., the effective pixel size.

Note that for CDI, performed in the Fraunhofer regime, typically a fully coherent beam is necessary. However, for ptychography it has been shown that due to the large redundancy of the measurement, additional information about the illuminating probe can be obtained [168], which also can include multiple modes that usually would be averaged to a single incoherent mode [171]. For a more mathematical treatment of coherence effects, we refer the reader to the concept of the mutual coherence function and complex degree of coherence, which give a measure of the correlation of individual realizations of a wave field [53, 133, 194].

\subsection{Dose and resolution}

The energy of x-rays is so high that ions and free electrons are created during absorption of x-rays by the photoelectric effect. These can lead to severe damage in all kind of matter. Especially in biological material, bond breaking and secondary effects induced by the ionizing radiation can destroy a sample $[32,117]$. Radiation damage will be stronger, if more energy is deposited in the material. To quantify the effects of ionizing radiation, the dose $D$ is introduced, defined as the absorbed energy per unit mass, with the unit gray: $1 \mathrm{~Gy}=1 \mathrm{~J} / \mathrm{kg}$. For a given photon fluence $F_{0}$, i.e., the time-integrated number of photons per unit area, LambertBeer's law states how much photons are transmitted in an object with linear attenuation coefficient $\mu_{L B}=2 k \beta$ up to a thickness $t$

$$
F(t)=F_{0} \mathrm{e}^{-\mu_{L B} t}
$$


For convenience, we will omit the subscript at $\mu_{L B}$ in the following. Hence, at the surface of the object, i.e., $t=0$, the number of photons absorbed is given by the derivative $-\left.\partial_{t} F(t)\right|_{t=0}=\mu F_{0}$, which is an upper bound of the absorption. With this expression the dose for photons of energy $h \nu$ can be written as [78]

$$
D=\frac{\mu F_{0} h \nu}{\rho_{m}},
$$

where $\rho_{m}$ is the mass density of the material. Note that this surface dose is also an upper estimate for the dose, and especially for thick objects the real dose can be lower.

If resolution is considered, one has to distinguish if the resolution is a function of an apparatus, e.g., limited by vibrations, or a function of the specimen [169]. Especially for the latter the dose will be an important factor. For a sample with a weak contrast, one needs a higher dose to obtain high resolution information, or noise will dominate the image. For the case of imaging in the Fraunhofer regime, the following relation for the required dose for imaging with a resolution of $d$ is $[78]$

$$
D_{\text {req. }}=\frac{\mu h \nu}{\rho_{m}} \frac{5^{2}}{r_{e}^{2} \lambda^{2}|\rho|^{2} d^{4}},
$$

where $\rho=k(\beta+\mathrm{i} \delta) / r_{e} \lambda$ is the complex electron density, $r_{e}$ is the classical electron radius and for the derivation a minimum signal to noise ratio (SNR) of 5 is assumed. Importantly, the above equation states that the required dose scales with the fourth power of the requested resolution. Hence, to obtain an image with a resolution improved by a factor of 10, a 10000 times higher dose is necessary. As radiation damage will limit the maximum tolerable dose of a biological object, the maximum achievable resolution for cryo-fixated samples is estimated to be at around $10 \mathrm{~nm}$ [78], unless the dose is applied in a time shorter than the time radiation damage needs to occur.

To determine the resolution of an image, different methods are used for this thesis. First of all, the measurements of known test structures can be used, to determine the resolution of the current experimental realization. Here, the resolution can be limited e.g., by the detector, partial incoherence or vibrations in the setup. The test structures can for example be objects with periodic structures of different sizes. Typically, a Siemens star or lines-and-spaces are used. If a structure of certain size can be observed with a good SNR, we will define its physical dimensions as the resolution of the imaging system. As the test structures in most cases are made of stable materials (e.g., nanostructured Tantal), radiation damage 
will not limit such a measurement.

For relevant biological samples, dose will be an issue and one way to estimate the resolution will be an analysis of the reconstructed image. The easiest way is to directly measure the size of relevant small features in the reconstruction or to measure the steepness of sharp edges. However, finding suitable features is not always possible, especially for images of biological specimens. Additionally, the resolution can depend on the direction. The Fourier-shell-correlation (FSC) is an established measure of resolution, known from electron microscopy [68]. It is defined for a 3D reconstruction, but can also be applied in a similar way for $2 \mathrm{D}$ images. To calculate the FSC, a reconstruction $X$ is split up into two independently reconstructed datasets $X_{1}$ and $X_{2}$, e.g., by taking every second angle of a tomographic scan. Subsequently, a normalized correlation of both datasets is calculated by [68]

$$
\operatorname{FSC}(|\boldsymbol{\xi}|)=\frac{<\tilde{X}_{1} \cdot \tilde{X}_{2}^{*}>}{\sqrt{<\left|\tilde{X}_{1}\right|^{2}>\cdot<\left|\tilde{X}_{2}\right|^{2}>}},
$$

where $\langle\cdot\rangle$ denotes the 3D angular average. Thus, the result will only depend on the distance $|\boldsymbol{\xi}|$ to the origin, where $\boldsymbol{\xi}$ is the coordinate vector in the Fourier space of the 3D volume. The resolution can then be estimated by the intersection of the FSC with a threshold curve. Different threshold curves are discussed in detail in [176]. For this work the so-called $1 / 2$ bit threshold curve, given by

$$
T_{1 / 2 \mathrm{bit}}(|\boldsymbol{\xi}|)=\frac{0.2071+1.9102 \cdot(n(|\boldsymbol{\xi}|))^{-\frac{1}{2}}}{1.2071+0.9102 \cdot(n(|\boldsymbol{\xi}|))^{-\frac{1}{2}}}
$$

will be used. Here, $n(|\boldsymbol{\xi}|)$ denotes the number of voxels in a shell with radius $|\boldsymbol{\xi}|$. The intersection of the FSC with this curve indicates the level, which is sufficient for interpretation of the data [176]. 



\section{Phase-retrieval approaches}

In the previous chapter we have derived analytical tools to describe the image formation in an x-ray imaging experiment. Classical x-ray imaging as introduced by Röntgen is based on measuring the absorption of a sample [150]. As the electric fields oscillate too fast, a direct measurement of the phase distribution $\phi\left(\mathbf{r}_{\perp}\right)$ is not possible. However, as $\delta$ is up to three orders of magnitude larger than $\beta$, the use of phase effects would enable superior imaging of weakly absorbing objects. In particular, the use of coherent illumination in combination with a free-space propagation enables the observation of interference effects that are caused by the phase shifts. Under plane wave illumination, the measurable intensity distribution is given by

$$
I\left(\mathbf{r}_{\perp}, z\right)=\left|\mathcal{D}_{z}\left(O\left(\mathbf{r}_{\perp}\right)\right)\right|^{2}
$$

with the object transmission function $O\left(\mathbf{r}_{\perp}\right)$ defined in Eq. (1.60) and the Fresnel propagator $\mathcal{D}_{z}$ from Eq. (1.24). Using the Fresnel scaling theorem, a cone-beam geometry obtained upon illumination with divergent spherical waves can be treated similarly in an effective geometry. As we will show in this chapter, phase retrieval with certain additional assumptions or multiple measurements enables the quantitative reconstruction of $O\left(\mathbf{r}_{\perp}\right)$ from the intensity distribution $I\left(\mathbf{r}_{\perp}, z\right)$.

In the direct-contrast regime edges will be enhanced, which allows direct observation of the structures. However, quantitative values that are necessary for computed tomography can only be obtained with suitable phase-retrieval algorithms. Especially in the holographic regime, consequently multiple interference fringes hinder the recognizability of the original structures, phase retrieval is an essential step to obtain an impression of the structure. The development and implementation of phase-retrieval algorithms constitute a large part of this work and in this chapter we will provide an overview of the different available methods. 


\subsection{Direct-contrast regime}

\subsubsection{Pure phase objects}

We consider again the simplified TIE (equation (1.76)) which was derived assuming no absorption and short propagation distances, i.e. large Fresnel numbers

$$
\nabla_{\perp}^{2} \phi\left(\mathbf{r}_{\perp}\right)=-\frac{k}{\Delta z}\left(\frac{I\left(\mathbf{r}_{\perp}, \Delta z\right)}{I_{0}}-1\right) .
$$

The right-hand side of the above equation can directly be measured. Thus, phase retrieval can be achieved by inverting the lateral Laplacian $\nabla_{\perp}^{2}$. To this end, we consider the derivative of a function $f(x)$ that can be written in Fourier space as:

$$
\begin{aligned}
\partial_{x} f(x) & =\frac{1}{\sqrt{2 \pi}} \int \partial_{x}\left(\tilde{f}(k) e^{\mathrm{i} k x}\right) d k \\
& =\frac{1}{\sqrt{2 \pi}} \int(\mathrm{i} k) \tilde{f}(k) e^{\mathrm{i} k x} d k
\end{aligned}
$$

Hence, the derivative operator can be formulated as a multiplication in Fourier space:

$$
\partial_{x}(\cdot)=\mathcal{F}^{-1}((\mathrm{i} k) \mathcal{F}(\cdot)),
$$

which can be easily generalized to include multiple derivative operations $m, n, p \in$ $\mathbb{N}$ in both lateral dimensions [133]:

$$
\left[\partial_{x}^{m}+\partial_{y}^{n}\right]^{p}(\cdot)=\mathcal{F}^{-1}\left(\left[\left(i k_{x}\right)^{m}+\left(i k_{y}\right)^{n}\right]^{p} \mathcal{F}(\cdot)\right)
$$

If one further allows $m, n$ and $p$ to be negative numbers, Eq. (2.5) can be used as a definition of the so-called pseudo differential operators [133]. In particular for $m=n=2$ and $p=-1$, the inverse lateral Laplacian can be defined:

$$
\nabla_{\perp}^{-2}=\mathcal{F}_{\perp}^{-1}\left(\frac{-1}{k_{x}^{2}+k_{y}^{2}} \mathcal{F}_{\perp}\right) .
$$

Equation (2.2) can thus be inverted using the above equation:

$$
\phi\left(\mathbf{r}_{\perp}\right)=2 \pi F^{1} \mathcal{F}_{\perp}^{-1}\left(\frac{\mathcal{F}_{\perp}\left(I / I_{0}-1\right)}{\left|\mathbf{k}_{\perp}^{\mathbf{0}}\right|^{2}}\right),
$$

where the unitless reciprocal coordinates $k_{x}^{0}=k_{x} \cdot p_{x}$ and $k_{y}^{0}=k_{y} \cdot p_{y}$ with the pixel sizes $p_{x}=p_{y}$ have been defined. Thus, the prefactor can be written in 
dependence on the Fresnel number for one pixel $F^{1}$. Note that in this equation a singularity at $\mathbf{k}_{\perp}^{0}=\mathbf{0}$ is present and low spatial frequencies only have little contrast transfer. As experimental phase-contrast images will contain noise and the pure-phase assumption may not be completely valid due to residual absorption, a regularization parameter $\alpha$ is introduced, to limit the amplification of low spatial frequencies:

$$
\phi\left(\mathbf{r}_{\perp}\right)=2 \pi F^{1} \mathcal{F}_{\perp}^{-1}\left(\frac{\mathcal{F}_{\perp}\left(I / I_{0}-1\right)}{\left|\mathbf{k}_{\perp}^{\mathbf{0}}\right|^{2}+\alpha}\right) .
$$

Hence, the image has to be filtered in Fourier space to reconstruct a quantitative phase-distribution. For too small regularization parameters $\alpha$, low spatial frequencies will be strongly amplified, resulting in a blurred image. For too large $\alpha$, the filter will be mainly a multiplication with $1 / \alpha$ and the frequency dependence will be negligible so that the reconstructed image will still show edge enhancement. The correct $\alpha$ can be chosen based on visual inspection of the reconstruction so that edge-enhancement effects in the image will be corrected for without blurring the image too much. With the use of the FFT, Eq (2.8) can be implemented as a fast algorithm for phase retrieval that is known as modified Bronnikov algorithm (MBA) [57]. Although it was originally proposed as a combined algorithm to perform phase retrieval and tomographic reconstruction simultaneously, in most cases it is more convenient to first reconstruct the phase distributions independently and to subsequently perform the tomographic reconstruction.

Figure 2.1 shows three simulated phase-contrast images to which the MBA was applied. For the simulation it was assumed that the object consists of a single material. Thus, phase $\phi\left(\mathbf{r}_{\perp}\right)$ and absorption $\mu\left(\mathbf{r}_{\perp}\right)$ are proportional to each other, with a ratio $\kappa=\delta / \beta=1000$ for the simulation. This situation accounts for a nearly transparent soft tissue object, whose maximum phase shift was chosen to be $\phi_{\max }=0.55 \mathrm{rad}$, hence the minimal transmission due to absorption was $I_{\min } / I_{0}=99.95 \%$. For the reconstruction, a regularization parameter $\alpha=4 \pi F^{1} \beta / \delta$ is chosen, as will be motivated in the next section. The intensity distributions are simulated with Poisson noise, corresponding to a total fluence of $5 \cdot 10^{4}$ photons/pixel. ${ }^{1}$ Note that the residual absorption acts as a regularization, i.e., the reconstruction of a simulated pure phase object would be more sensitive to the noise. For smaller Fresnel numbers, the contrast transfer increases. Hence, the reconstruction contains less noise which can be seen e.g., in Fig. 2.1(e). However, as becomes clear when considering the power spectral density (PSD) of the recon-

\footnotetext{
1 In practice, intensity values are scaled with the number of photons per pixel, such that a value of 1 corresponds to $5 \cdot 10^{4}$ photons, a value of 0.1 to $5 \cdot 10^{3}$ and so on.
} 


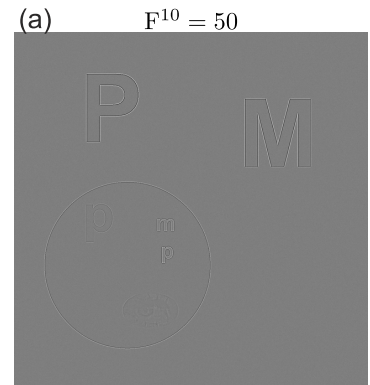

(d)

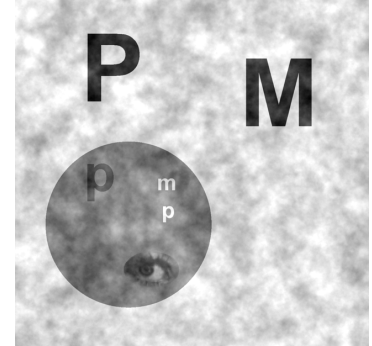

(g)

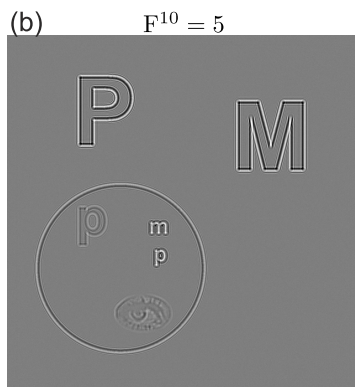

(e)

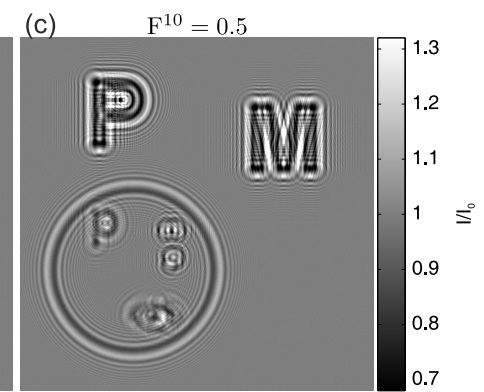

(f)
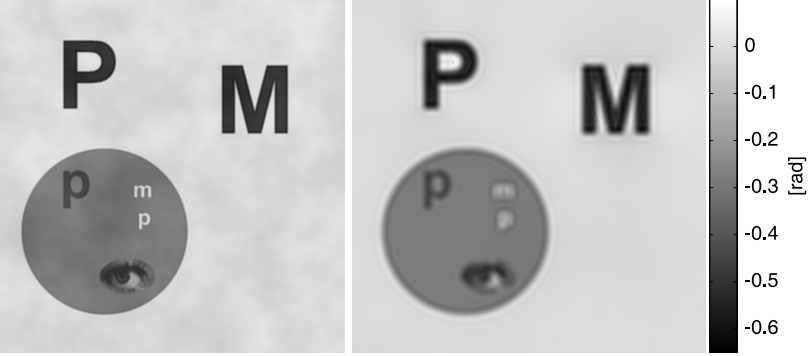

(i)

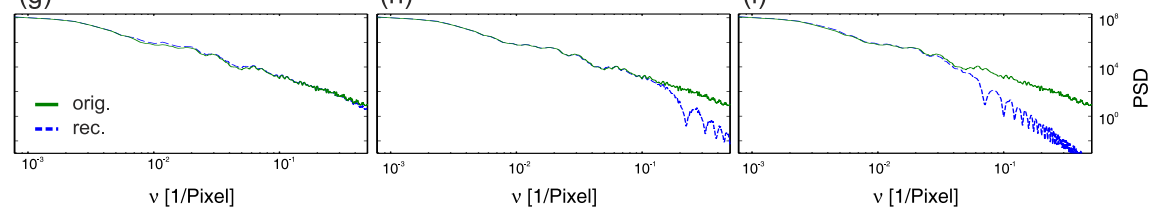

Figure 2.1: Application of MBA to simulated data of a weakly absorbing, homogeneous object: $(a, b, c)$ show simulated, noisy intensity distributions $I\left(\mathbf{r}_{\perp}\right)$ for different Fresnel numbers for which the phase retrieval was performed, to yield $(d, e, f)$ the reconstructed phase distribution $\phi\left(\mathbf{r}_{\perp}\right)$. $(g, h, f)$ The azimuthally averaged PSDs of the reconstruction and of the original object show a stronger deviation at high spatial frequencies for smaller Fresnel numbers with significant minima at frequencies corresponding to the zeros in the CTF.

struction (Fig. 2.1(g) to (i)) compared to the original PSD, the approximation of small propagation distances $\Delta z$ (more correctly large Fresnel numbers) is not valid anymore for the high spatial frequencies at smaller Fresnel numbers. Note that zeros in the PCTF are observable in the PSD of the reconstruction for $F^{10}=5$ and $F^{10}=0.5$. For the resulting image impression, these zeros express themselves as a blurring of the reconstruction.

The Fresnel number in Eq. (2.8) just acts as a global scaling factor. Thus, for the case of limited temporal coherence, i.e., a large bandwidth $\Delta \lambda / \lambda$, different wavelengths will contribute similarly and a sharp reconstruction for an effective 
mean wavelength $\lambda_{\text {eff }}$ can be obtained, whose absolute phase-shift values may be systematically biased.

\subsubsection{Single material objects}

For the derivation of the MBA it was assumed that we have a pure-phase object, which introduced a singularity in the reconstruction formula raising the need for regularization. Another method to reconstruct phase-contrast images in the directcontrast regime was proposed by Paganin et al. [131]. For their algorithm, it is assumed that an object consists of a single material, so that phase and absorption (equations (1.61) and (1.62)) are proportional to each other. In the literature the latter effect is often called phase-attenuation duality [28] and we will also refer to those objects as homogeneous objects. If one defines the projected thickness of an object $T\left(\mathbf{r}_{\perp}\right)$, it is

$$
\begin{aligned}
& \phi\left(\mathbf{r}_{\perp}\right)=-k \int_{-d}^{0} \delta(\mathbf{r}) \mathrm{d} z=-k \delta T\left(\mathbf{r}_{\perp}\right), \\
& \mu\left(\mathbf{r}_{\perp}\right)=2 k \int_{-d}^{0} \beta(\mathbf{r}) \mathrm{d} z=2 k \beta T\left(\mathbf{r}_{\perp}\right),
\end{aligned}
$$

where $\delta$ and $\beta$ are the material constants that do not change within the object. Using the above equation and the projection approximation (Eq. (1.58)), the intensity in the object exit-plane can be written as

$$
\begin{aligned}
I\left(\mathbf{r}_{\perp}, 0\right) & =\left|\psi\left(\mathbf{r}_{\perp}, 0\right)\right| \\
& =I_{0} \mathrm{e}^{-\mu_{L B} T\left(\mathbf{r}_{\perp}\right)},
\end{aligned}
$$

where a plane wave illumination has been assumed, and the definition of the linear absorption coefficient $\mu_{L B}=2 k \beta$ known from Lambert-Beer's law was used. These expressions for $\phi\left(\mathbf{r}_{\perp}, 0\right)$ and $I\left(\mathbf{r}_{\perp}, 0\right)$ can be substituted in the TIE (Eq. (1.75)), yielding

$$
I_{0} \nabla_{\perp} \cdot\left(\mathrm{e}^{-\mu T\left(\mathbf{r}_{\perp}\right)} \nabla_{\perp}\left(\delta T\left(\mathbf{r}_{\perp}\right)\right)\right)=\frac{\partial I(\mathbf{r})}{\partial z} .
$$

If small propagation distances are assumed again, the differential quotient in this equation can be approximated as before, and after simplifications, Eq. (2.12) can be written as

$$
\left(1-\frac{\delta \Delta z}{\mu} \nabla_{\perp}^{2}\right) \mathrm{e}^{-\mu T\left(\mathbf{r}_{\perp}\right)}=\frac{I\left(\mathbf{r}_{\perp}, \Delta z\right)}{I_{0}} .
$$


This differential equation can be solved using the pseudo differential operators. After applying the logarithm, the result will give an expression for the projected thickness $T\left(\mathbf{r}_{\perp}\right)$ that can be substituted into (2.9) to calculate the phase via [131]

$$
\phi\left(\mathbf{r}_{\perp}, 0\right)=\frac{\delta}{2 \beta} \cdot \ln \mathcal{F}_{\perp}^{-1}\left(\frac{\mathcal{F}_{\perp}\left(I\left(\mathbf{r}_{\perp}, \Delta z\right) / I_{0}\right)}{\left(1+\frac{\delta}{2 k \beta} \Delta z \mathbf{k}_{\perp}^{2}\right)}\right) .
$$

This equation again does not depend on the actual values of $\lambda, z$ or the pixel size $p$, but on the Fresnel number $F^{1}$ and only the ratio $\kappa=\delta / \beta$ matters instead of $\delta$ and $\beta$ alone

$$
\phi\left(r_{\perp}, 0\right)=\frac{\kappa}{2} \cdot \ln \mathcal{F}_{\perp}^{-1}\left(\frac{\mathcal{F}_{\perp}\left(I\left(\mathbf{r}_{\perp}, \Delta z\right) / I_{0}\right)}{\left(1+\frac{\kappa}{4 \pi F^{1}}\left|\mathbf{k}^{\mathbf{0}}\right|^{2}\right)}\right) .
$$

In the following we will refer to the reconstruction algorithm defined by this equation as the single material object reconstruction (SMO) or Paganin reconstruction. Note that for $\alpha=4 \pi F^{1} \kappa^{-1}$ the filter applied in Fourier space of Eq. (2.8) is the same as in Eq. (2.15). Thus, if a reconstruction with the MBA is performed, the presence of a single-material is implicitly assumed. It has been shown that the Paganin reconstruction is superior for strongly absorbing objects [24], but for weak objects both, the SMO and MBA, are very similar. Note that the single-material assumption can be violated, as long as the ratio $\kappa$ remains (approximately) constant, e.g., for a material with similar stoichiometry but different densities. In practice, if the assumption to the object is violated, blurring effects or remaining edge enhancement will be visible, depending on the actual choice of the regularization parameter $\alpha$ or equivalently the ratio $\kappa=\delta / \beta$. For this reason, the parameters will be chosen based on visual inspection of the images, so that no significant blurring occurs and edge enhancement is reduced as much as possible. For the simulated object in Fig. 2.1, which consists of a single material, no significant differences between SMO and MBA can be observed.

\subsubsection{Bronnikov aided correction}

If edge enhancement is removed with one of the algorithms presented so far and the single material assumption is violated, significant blurring effects can be observed in a reconstructed phase map. One method to obtain sharper reconstructions can be derived by considering the simplified TIE (Eq. (1.77)) once more that was also 
used to derive the MBA

$$
I\left(\mathbf{r}_{\perp}, \Delta z\right)=I_{0}\left(1+\frac{\Delta z}{k} \nabla_{\perp}^{2} \phi\left(\mathbf{r}_{\perp}, 0\right)\right) .
$$

In the derivation of the above equation a plane wave illumination with intensity $I_{0}$ and no absorption of the object is assumed. However, due to the regularization introduced for the MBA, it is implicitly assumed that the object absorbs the radiation. For a weakly and almost homogeneously absorbing object, the uniform illumination $I_{0}$ can be replaced by the absorption image $I_{a}\left(\mathbf{r}_{\perp}\right):=\mathrm{e}^{-\mu\left(\mathbf{r}_{\perp}\right)}[136]$. Thus, Eq. (2.16) can be solved for $I_{a}$ yielding

$$
I_{a}\left(\mathbf{r}_{\perp}\right)=\frac{I\left(\mathbf{r}_{\perp}, \Delta z\right)}{1-\frac{\Delta z}{k} \nabla_{\perp}^{2} \phi\left(\mathbf{r}_{\perp}\right)}
$$

The MBA algorithm delivers an approximation $\phi^{\dagger}\left(\mathbf{r}_{\perp}\right)$ to the real phase distribution $\phi\left(\mathbf{r}_{\perp}\right)$ that can be used to calculate a hypothetical pure phase-contrast image $C\left(\mathbf{r}_{\perp}\right)$

$$
C\left(\mathbf{r}_{\perp}\right)=1-\gamma \nabla^{2} \phi^{\dagger}\left(\mathbf{r}_{\perp}\right),
$$

where the prefactor $\Delta z / k$ is replaced by a parameter $\gamma$. This replacement is done, as the regularization parameter $\alpha$ is chosen based on visual inspection, which can be compensated by the right choice of $\gamma$. The pure phase-contrast image $C\left(\mathbf{r}_{\perp}\right)$ can then be used to correct the measured intensity $I\left(\mathbf{r}_{\perp}, \Delta z\right)$

$$
I_{a}\left(\mathbf{r}_{\perp}\right)=\frac{I\left(\mathbf{r}_{\perp}, \Delta z\right)}{1-\gamma \nabla_{\perp}^{2} \phi^{\dagger}\left(\mathbf{r}_{\perp}\right)},
$$

known as the so-called Bronnikov aided correction (BAC) that was first introduced by de Witte et al. [187]. In practice, the reconstruction works as follows: An approximate phase distribution $\phi^{\dagger}$ is reconstructed by choosing the correct $\alpha$, being so small that edge-enhancement is removed and the image is not blurred. Subsequently, a correction image $C$ is calculated by choosing $\gamma$ so large that edgeenhancement is reduced but not inverted. As implicitly a phase-attenuation duality is assumed, the reconstructed $I_{a}\left(\mathbf{r}_{\perp}\right)$ can be used to calculate an effective phase distribution by

$$
\phi\left(\mathbf{r}_{\perp}\right)=\log \left(I_{a}\right) \cdot \kappa / 2
$$

with the ratio $\kappa=\delta / \beta$.

The derivation of the $\mathrm{BAC}$ is quite empirical and for the reconstruction of a sim- 


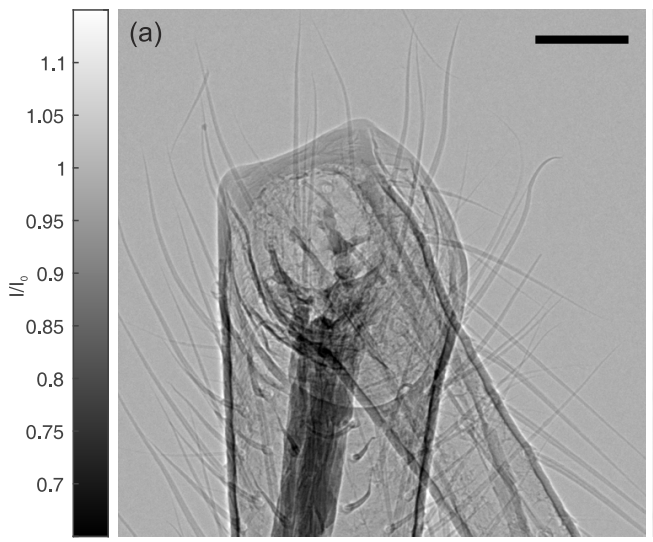

(b)

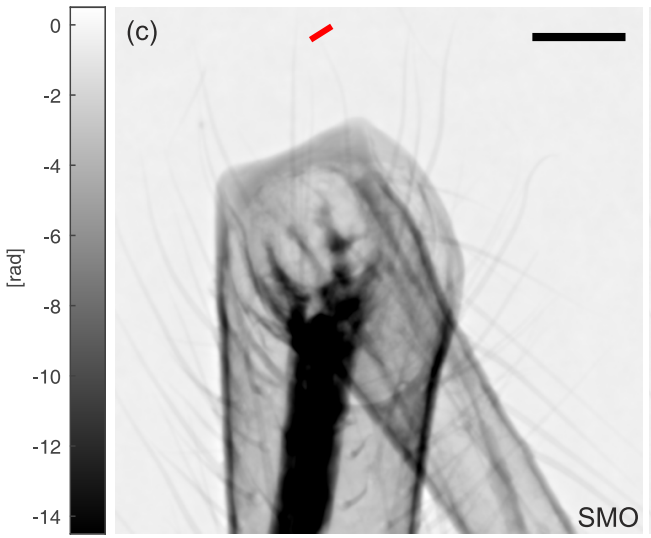

(d)

MBA

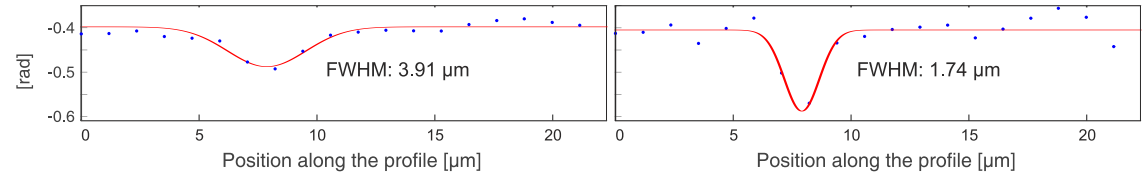

Figure 2.2: Application of nearfield phase-retrieval algorithms to experimental data: (a) An empty-beam corrected phase-contrast image $I / I_{0}$ of a hairy spider leg, recorded so that the Fresnel number is $F^{10} \simeq 51$. (b) shows the MBA reconstruction for $\alpha=4.5 \cdot 10^{-3}$. (c) shows the SMO phase reconstruction with $\kappa=4 \pi F^{1} / \alpha \simeq 142$ together with a profile of the reconstructed phase values plotted along the red line through a single hair. In (d) the BAC reconstruction with $\gamma=0.15 / F^{1}$ is shown, where the absorption $I_{a}$ values have been used to calculate an effective phase distribution $\phi$ with Eq. (2.20) and the same profile as in (c) is plotted below. Note that for (b) the colorbar from (c) and (d) shows the correct gray values. All scale bars have a length of $100 \mu \mathrm{m}$. 
ulated single-material object, no benefit compared to MBA or SMO is observable (data not shown). However, for experimental data the resolution in the reconstructions is increased [187]. Figure 2.2 shows a measured phase-contrast image of a hairy spider leg with different phase-retrieval algorithms for the direct-contrast regime applied. Although the measurement shows very fine structures, the phase maps retrieved with MBA and SMO appear blurred. This blurring could of course be reduced by choosing a larger regularization parameter $\alpha$. However, for larger $\alpha$ the edge enhancement effects are not fully compensated, so that quantitative values would be biased. Note that between MBA and SMO, a small difference in the absolute values can be observed, although structural details are the same. The BAC reconstruction in contrast shows a much crisper image. The effect can be quantified, e.g., by evaluating the width of a single hair. Figure 2.2(c) and (d) additionally show two profiles of the reconstructed values along a line that crosses a hair together with a Gaussian fit. While in the SMO and also in the MBA reconstruction, a full width at half maximum (FWHM) of about $3.9 \mu \mathrm{m}$ can be measured, the BAC reconstruction yields a FWHM of just $1.7 \mu \mathrm{m}$ and thus the resolution is estimated to be at least twice as good as compared to the other reconstructions.

\subsection{Holographic regime}

In the direct-contrast regime the main benefit of applying phase-retrieval algorithms is the obtainment of quantitative values, which are needed, e.g., for the application of tomography. However, for smaller Fresnel numbers, interference effects become stronger and multiple fringes can be observed in an image (cf. Fig. 1.6). In this imaging regime, phase retrieval is a necessary step to gain an understanding for the real-space structure of the specimen. In particular at high spatial-frequencies, responsible for fine details in an image, information about the original structure will be lost first.

\subsubsection{Holographic reconstruction}

The simplest idea of a holographic reconstruction goes back to Gabor and his proposal of holography with a coherent electron beam [51]. His idea was to record the intensity pattern on a photographic plate, develop it and to shine on the plate with an illumination that imitates the original one. Formally, after propagating 

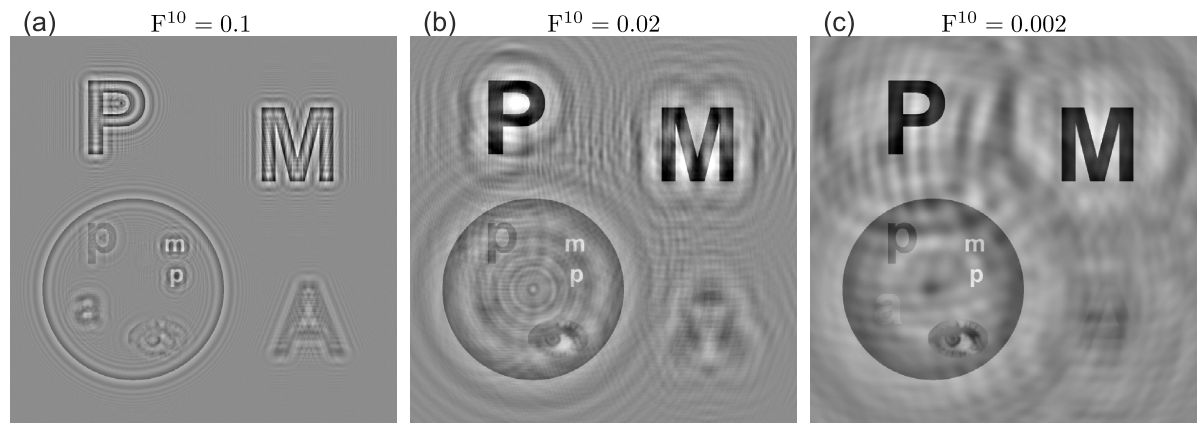

Figure 2.3: Holographic reconstruction for phase retrieval of an inhomogeneous object as in Fig. 1.6. (a), (b) and (c) show the phase of the wave field $\psi^{\prime}\left(\mathbf{r}_{\perp}, 0\right)=$ $\mathcal{D}_{-z}\left(I\left(\mathbf{r}_{\perp}, z\right)\right)$ obtained by numerical Fresnel back propagation of the simulated intensity distributions, shown in Fig. 1.6 for different Fresnel numbers. It can be clearly seen that for smaller Fresnel numbers, the reconstruct values closer to quantitative truth, as the overlaying twin image will be even more holographic. Note that the purely absorbing A's have only little influence if small Fresnel numbers are present. The gray values range from -0.5 (black) to +0.4 (white).

the distance $z$, the intensity can be written as

$$
I\left(\mathbf{r}_{\perp}, z\right)=\left|\mathcal{D}_{z}\left(\psi\left(\mathbf{r}_{\perp}, 0\right)\right)\right|^{2}
$$

for an arbitrary exit wave $\psi\left(\mathbf{r}_{\perp}, 0\right)$. We assume the wave function to consist of a reference wave $\psi_{0}$ and scattered wave $\psi_{s}$

$$
\psi\left(\mathbf{r}_{\perp}, 0\right)=\psi_{0}\left(\mathbf{r}_{\perp}, 0\right)+\psi_{s}\left(\mathbf{r}_{\perp}, 0\right)
$$

This situation is given for example by a weakly scattering object in the projection approximation, where $O\left(\mathbf{r}_{\perp}\right) \simeq 1-\mathrm{i} k \delta\left(\mathbf{r}_{\perp}\right)-k \beta\left(\mathbf{r}_{\perp}\right)$. In the following, we will regard paraxial wave fields $\psi^{\prime}$ with the fast oscillating term $\mathrm{e}^{\mathrm{i} k z}$ separated. For a normalized plane wave illumination $\psi_{0}^{\prime}\left(\mathbf{r}_{\perp}, 0\right)=1$, the propagated version will still be unity, so that with this abbreviation, Eq. (2.21) reads

$$
\begin{aligned}
I\left(\mathbf{r}_{\perp}, z\right) & =\left|\psi_{0}^{\prime}\left(\mathbf{r}_{\perp}, z\right)+\psi_{s}^{\prime}\left(\mathbf{r}_{\perp}, z\right)\right|^{2} \\
& =1+\psi_{s}^{\prime}\left(\mathbf{r}_{\perp}, z\right)+\psi_{s}^{\prime *}\left(\mathbf{r}_{\perp}, z\right)+\left|\psi_{s}^{\prime}\left(\mathbf{r}_{\perp}, z\right)\right|^{2} .
\end{aligned}
$$

As the scattered wave is assumed to be much weaker than the reference wave $\psi_{0}^{\prime}$, the quadratic term in Eq. (2.23) can be neglected. To obtain the original object exit wave $\psi_{s}^{\prime}\left(\mathbf{r}_{\perp}, 0\right)$, the measured hologram has to be back propagated, either by 
physically illuminating a holographic plate as in [51] or by numerical propagation of a measured digital image. Formally, this propagation step can be written as

$$
\begin{aligned}
\mathcal{D}_{-z}\left(I\left(\mathbf{r}_{\perp}, z\right)\right) & \approx \mathcal{D}_{-z}\left(1+\psi_{s}^{\prime}\left(\mathbf{r}_{\perp}, z\right)+\psi_{s}^{\prime *}\left(\mathbf{r}_{\perp}, z\right)\right) \\
& =1+\psi_{s}^{\prime}\left(\mathbf{r}_{\perp}, 0\right)+\mathcal{D}_{-z}\left(\psi_{s}^{\prime *}\left(\mathbf{r}_{\perp}, z\right)\right) .
\end{aligned}
$$

Hence, the back propagated intensity will directly contain the scattered wave field $\psi_{s}^{\prime}\left(\mathbf{r}_{\perp}, 0\right)$, overlaid by the so-called twin image $\mathcal{D}_{-z}\left(\psi_{s}^{\prime}\left(\mathbf{r}_{\perp}, z\right)\right)$. It can be shown that the back propagation of the complex conjugate of the function corresponds to a virtual image, recorded at twice the propagation distance $z$ [133]. Thus, the twin image will disturb the reconstruction, as illustrated in Fig. 2.3. For large Fresnel numbers the twin-image is a hologram that still shows much similarity to the real object, where for smaller Fresnel numbers the twin image will be more holographic so that the disturbance by the twin image will have less influence. While high frequencies like edges in the images are reconstructed quite well for all Fresnel numbers, especially the low spatial frequencies are strongly disturbed at large Fresnel numbers and the improvement at smaller $F^{10}$ can be best seen at the circle or the large letters in Fig. 2.3(c).

\subsubsection{CTF-based phase-retrieval}

The holographic reconstruction can give a first impression of the recorded structure. However, quantitative information is lost and the twin-image problem introduces strong artifacts. A widely used technique for weakly absorbing and weakly scattering objects is based on the CTF (Eq. (1.71)). First, we once again regard the special case of a pure phase object with $\mu\left(\mathbf{r}_{\perp}\right)=0$. The CTF in this case reads

$$
\tilde{I}_{\text {approx. }}\left(\boldsymbol{\nu}_{\perp}, z\right)=\delta_{D}\left(\boldsymbol{\nu}_{\perp}\right)+2 \tilde{\phi}\left(\boldsymbol{\nu}_{\perp}\right) \sin \chi .
$$

This equation predicts the intensity for a given Fresnel number and phase distribution $\tilde{\phi}$. We are looking for a phase distribution that minimizes the error between an experimentally measured image $\tilde{I}_{\exp }$ and the intensity given by Eq. (2.25). Hence, to find a suitable phase distribution $\phi\left(\boldsymbol{\nu}_{\perp}\right)$ we can define an error metric, which has to be minimized. To allow for measurements of the intensity distribution for different Fresnel numbers, we define the following cost function for 
the measurement at $N$ different propagation distances [192]

$$
\begin{aligned}
S_{c} & =\frac{1}{N} \sum_{m=1}^{N} \int_{\mathbb{R}^{2}}\left|\tilde{I}_{\exp }\left(\boldsymbol{\nu}_{\perp}, z_{m}\right)-\tilde{I}_{\text {approx. }}\left(\boldsymbol{\nu}_{\perp}, z_{m}\right)\right|^{2} \mathrm{~d} \boldsymbol{\nu}_{\perp} \\
& =\frac{1}{N} \sum_{m=1}^{N} \int_{\mathbb{R}^{2}}\left|\tilde{I}_{\exp }\left(\boldsymbol{\nu}_{\perp}, z_{m}\right)-\delta_{D}\left(\boldsymbol{\nu}_{\perp}\right)+2 \tilde{\phi}\left(\boldsymbol{\nu}_{\perp}\right) \sin \chi_{m}\right|^{2} \mathrm{~d} \boldsymbol{\nu}_{\perp}
\end{aligned}
$$

with the argument of the sine function that can be written so that it only depends on the Fresnel number

$$
\chi_{m}=\pi \lambda z\left(\nu_{x}^{2}+\nu_{y}^{2}\right)=\pi\left|\boldsymbol{\nu}^{0}\right|^{2} / F_{m}^{1} .
$$

The cost function (2.26) will be minimal for $\partial S_{c} / \partial \tilde{\phi}=0$, which yields an equation that can be solved for $\tilde{\phi}$

$$
\tilde{\phi}\left(\boldsymbol{\nu}_{\perp}\right)=\frac{\sum_{m} \tilde{I}_{\mathrm{exp}}\left(\boldsymbol{\nu}_{\perp}, z_{m}\right) \cdot \sin \chi_{m}}{\sum_{m} 2 \sin ^{2} \chi_{m}} .
$$

The denominator in the above equation always disappears for $\boldsymbol{\nu}_{\perp}=\mathbf{0}$ and, depending on the Fresnel numbers $F_{m}^{1}$, can disappear for other spatial frequencies, too. Thus, as for the direct-contrast regime a regularization parameter $\alpha\left(\boldsymbol{\nu}_{\perp}\right)$ is introduced, so that the phase distribution in real space is given by

$$
\phi\left(\mathbf{r}_{\perp}\right)=\mathcal{F}_{\perp}^{-1}\left(\frac{\sum_{m} \mathcal{F}_{\perp}\left(I_{\exp }\left(\mathbf{r}_{\perp}, z_{m}\right)-1\right) \cdot \sin \chi_{m}}{\sum_{m} 2 \sin ^{2} \chi_{m}+\alpha\left(\boldsymbol{\nu}_{\perp}\right)}\right) .
$$

In the holographic regime, $\alpha\left(\boldsymbol{\nu}_{\perp}\right)$ is chosen in order to regularize low and high spatial frequencies differently. This is motivated by the fact that low spatial frequencies have to be regularized mainly due to lack of contrast transfer and residual absorption, whereas high spatial frequencies mainly need to be regularized due to zeros in the PCTF that will have less influence if multiple intensity measurements are carried out. In practice, $\alpha$ is built to reach a low limit $\alpha_{1}$ and a high limit $\alpha_{2}$

$$
\alpha\left(\boldsymbol{\nu}_{\perp}\right)=\alpha_{1} \cdot f\left(\left|\boldsymbol{\nu}_{\perp}\right|\right)+\alpha_{2} \cdot\left(1-f\left(\left|\boldsymbol{\nu}_{\perp}\right|\right)\right)
$$

with the transition between them being realized by an error function [35]

$$
f\left(\left|\boldsymbol{\nu}_{\perp}\right|\right)=\frac{1}{2}\left(1-\operatorname{erf}\left(\frac{\boldsymbol{\nu}_{\perp}^{2}-\nu_{c u t}}{\sigma_{c u t}}\right)\right)
$$




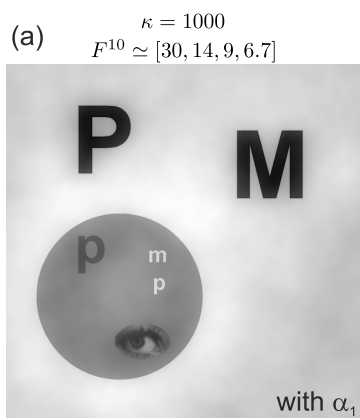

(b) $\begin{gathered}\kappa=1000 \\ F^{10} \simeq[0.26,0.2,0.17,0.14]\end{gathered}$

$\kappa=10$
(c) $F^{10} \simeq[0.26,0.2,0.17,0.14]$
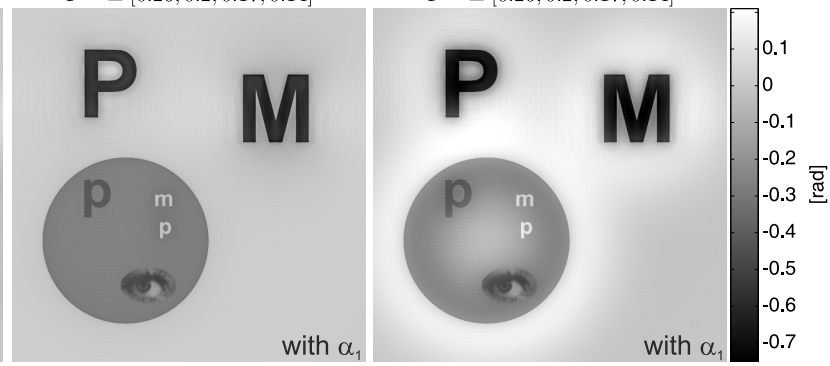

(d)

(e)

(f)

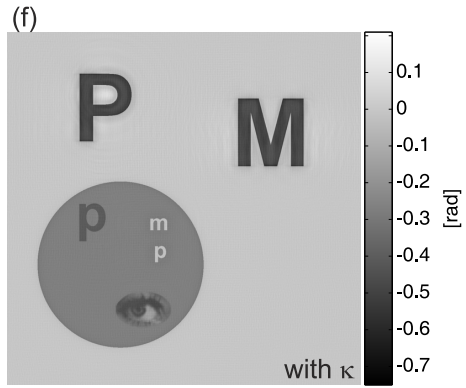

with $\kappa$
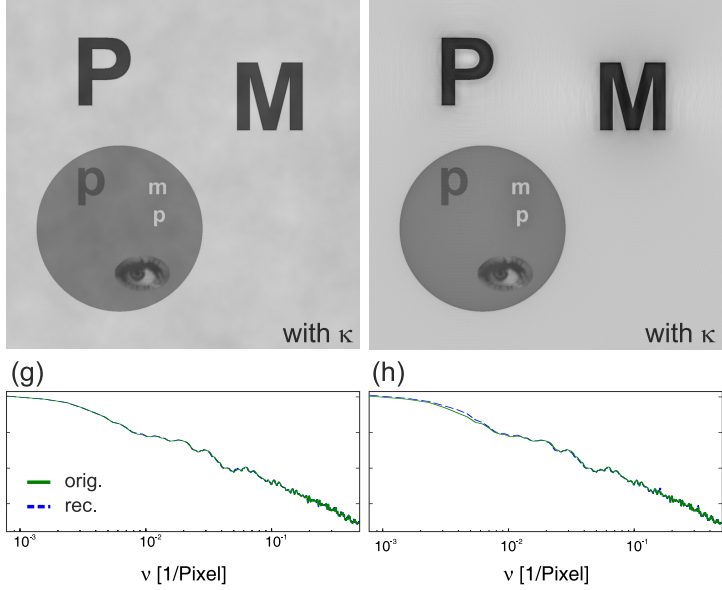

(h)

(i)

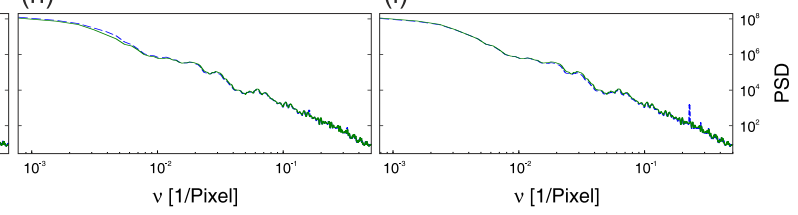

Figure 2.4: CTF-based phase-retrieval for homogeneous objects with $\kappa=1000$ and $\kappa=10$ and for different sets of Fresnel numbers, listed on top. (a,b,c) show the reconstructed phase distributions obtained with Eq. (2.29). Regularization parameters $\alpha_{1}$ were chosen based on minimizing the difference to the real phase distribution. (d,e,f) show the reconstructed phase distributions obtained with Eq. (2.33) where the known $\kappa$ ratio was used for the reconstruction. (g,h,i) show the azimuthally averaged PSDs for the reconstructions obtained in $(d, e, f)$ in comparison to the original PSDs. Note that no deviations due to zeros in the CTF can be observed.

where the point of inflection $\nu_{c u t}$ is at the first maximum of the $\mathrm{CTF}$ and $\sigma_{\text {cut }}$ determines how fast the transition will be. If not stated differently, we will choose $\sigma_{c u t}=0.01$ periods per pixel for CTF-based phase-retrievals in this thesis.

The assumption of a pure-phase object can be relaxed, if again a homogeneous object, consisting of a single material, is regarded. For a given ratio $\kappa=\delta / \beta$ the absorption $\tilde{\mu}\left(\boldsymbol{\nu}_{\perp}\right)$ can be written in dependence on the phase and the CTF thus 
reads

$$
\begin{aligned}
\tilde{I}_{\text {approx. }}\left(\boldsymbol{\nu}_{\perp}, z_{m}\right) & =\delta_{D}\left(\boldsymbol{\nu}_{\perp}\right)+2 \tilde{\phi}\left(\boldsymbol{\nu}_{\perp}\right) \sin \chi_{m}+\frac{2}{\kappa} \tilde{\phi}\left(\boldsymbol{\nu}_{\perp}\right) \cos \chi_{m} \\
& =\delta_{D}\left(\boldsymbol{\nu}_{\perp}\right)+2 \tilde{\phi}\left(\boldsymbol{\nu}_{\perp}\right)\left(\sin \chi_{m}+\frac{1}{\kappa} \cos \chi_{m}\right) .
\end{aligned}
$$

The next steps will be exactly the same as in the pure-phase case presented above. After defining a cost function and minimizing it with respect to $\tilde{\phi}$, the resulting reconstruction formula for the phase distribution in real space reads [172]

$$
\phi\left(\mathbf{r}_{\perp}\right)=\mathcal{F}_{\perp}^{-1}\left(\frac{\sum_{m} \mathcal{F}_{\perp}\left(I_{\exp }\left(\mathbf{r}_{\perp}, z_{m}\right)-1\right) \cdot\left(\sin \chi_{m}+\frac{1}{\kappa} \cos \chi_{m}\right)}{\sum_{m} 2\left(\sin \chi_{m}+\frac{1}{\kappa} \cos \chi_{m}\right)^{2}+\alpha\left(\boldsymbol{\nu}_{\perp}\right)}\right) .
$$

Note that due to the $\cos \chi_{m}$ term, the singularity at $\boldsymbol{\nu}_{\perp}=\mathbf{0}$ is not present anymore. Hence, for a practical implementation, the regularization parameter $\alpha_{1}$ is set to zero in case a $\kappa$ ratio is given, as $\kappa$ already serves as regularization for the low frequencies. There are also methods that do not assume a homogeneous object, which leads to two reconstruction formulas: one for the phase and one for the amplitudes [192]. However, in practice this kind of reconstruction is often not well constrained and the CTF reconstructions presented so far will perform better.

Figure 2.4 shows the application of Eq. (2.29) and (2.33) to simulated data for homogeneous objects that only have little absorption $(\kappa=1000)$ and for up to $5 \%$ absorption $(\kappa=10)$. Poisson noise corresponding to $5 \cdot 10^{4}$ photons per pixel is added as before. For each reconstruction, four different intensity distributions were simulated at optimized propagation distances that can be calculated via [192]

$$
z_{\text {opt. }}[\mathrm{mm}]=\frac{1}{\lambda[\AA]}\left(\left(c_{2} \cdot p^{2}+c_{1} \cdot p+c_{0}\right) \cdot j-d_{1} p+d_{0}\right) \quad j \in \mathbb{N} .
$$

Here, $p$ has to be the pixel size in micrometers and the constants $c_{i}$ and $d_{i}$ are determined numerically to: $c_{2}=38.4, c_{1}=0.4, c_{0}=-0.12, d_{1}=9.84$ and $d_{0}=4.67$. For the simulation in Fig. 2.4 two different Fresnel number regimes are covered by using Eq. (2.34) for $\Delta x=1 \mu \mathrm{m}$ and $j \in[1,2,3,4]$ to obtain intensities in the direct contrast regime and by $j \in[100,130,150,190]$ to be in the holographic regime. Note that any choice of $j$ leads to optimum propagation distances. If these distances are chosen, the high spatial frequencies will add up so that minima and maxima cancel each other out in an optimal way [192]. 
Hence, the regularization parameter $\alpha_{2}$ can be chosen as small as possible. For the reconstructions in Fig. $2.4 \alpha_{2}=10^{-16}$ is chosen. The regularization parameter for the low frequencies $\alpha_{1}$ was automatically chosen based on minimizing the deviation between reconstructed and original phase. Of course, this strategy can only be applied for simulated data. For experimental data, $\alpha_{1}$ will be chosen similar to the nearfield case based on visual inspection. As can be seen in Fig. 2.4, the reconstruction assuming a homogenous object, given by Eq. (2.33), is better suited for the phase retrieval, especially as the low frequencies are better constrained. However, both reconstructions show only minimal artifacts, especially when compared to the holographic reconstructions shown in Fig. 2.3. Note that if residual beam variations are present in the image, which may be caused by a temporally changing illumination, they will disturb the reconstruction. As these variations mainly occur at the low spatial frequencies, they can be suppressed by choosing a larger $\alpha_{1}$ [87]. The suppression will also affect the low frequencies of the reconstruction but the results are often of sufficient quality to obtain a first impression or to serve as initial estimate for other phase-retrieval approaches.

Although the reconstruction is only demonstrated for two sets of Fresnel numbers, the algorithm is not limited to a specific regime and the reconstruction works well as long as the oscillatory part of the CTF is well sampled. For very small Fresnel numbers, the FOV in real space has to be increased, e.g., by replicative padding, to ensure proper sampling of the CTF in Fourier space. Note that the CTF-based phase-retrieval also delivers good results in the direct contrast regime. So, as long as the temporal coherence is adequate, which is the case at synchrotron facilities, it will be beneficial to use the CTF reconstruction rather than MBA or SMO. 


\subsubsection{Holo-TIE}

The holographic reconstruction and the CTF-based phase-retrieval have a significant drawback, as they may be disturbed by the twin image and assume the object to be only weakly scattering and absorbing. If assumptions are fulfilled, especially the CTF can deliver good reconstructions and due to the incorporation of multiple distances, the twin-image problem can be solved quite well. Another holographic phase-retrieval method that was established during this work is based on solving the TIE in an arbitrary plane, e.g., the detector plane. The phase distribution and the measured intensity are used to build a wave-function in the detection plane, which can be propagated back to the object plane [88]. A main benefit of this technique is that no restrictive assumptions to the object are made and thus, in theory, arbitrary objects can be reconstructed.

\subsubsection{The ideal case}

To derive the aforementioned phase-retrieval method, we once again consider the TIE (Eq. (1.75))

$$
\nabla_{\perp} \cdot\left(I(\mathbf{r}) \nabla_{\perp} \phi(\mathbf{r})\right)=-k \frac{\partial I(\mathbf{r})}{\partial z} .
$$

We introduce an auxiliary function $\xi$ such that [166]

$$
I(\mathbf{r}) \nabla_{\perp} \phi(\mathbf{r}) \approx \nabla_{\perp} \xi(\mathbf{r})
$$

Hence, the TIE can be formally solved with the pseudo differential operators introduced in section 2.1.1

$$
\xi=-k \nabla_{\perp}^{-2}\left(\frac{\partial I(\mathbf{r})}{\partial z}\right) .
$$

Resubstituting the definition of Eq. (2.36) yields

$$
I(\mathbf{r}) \nabla_{\perp} \phi(\mathbf{r})=-k \nabla_{\perp}\left[\nabla_{\perp}^{-2}\left(\frac{\partial I(\mathbf{r})}{\partial z}\right)\right] .
$$

Dividing both sides by $I(\mathbf{r})$, applying a lateral gradient $\nabla_{\perp}$ and using the inverse Laplacian, a solution for $\phi$ is given by [132]

$$
\phi(\mathbf{r})=-k \nabla_{\perp}^{-2}\left(\nabla_{\perp}\left\{\frac{1}{I(\mathbf{r})} \nabla_{\perp}\left[\nabla_{\perp}^{-2}\left(\frac{\partial I(\mathbf{r})}{\partial z}\right)\right]\right\}\right) .
$$




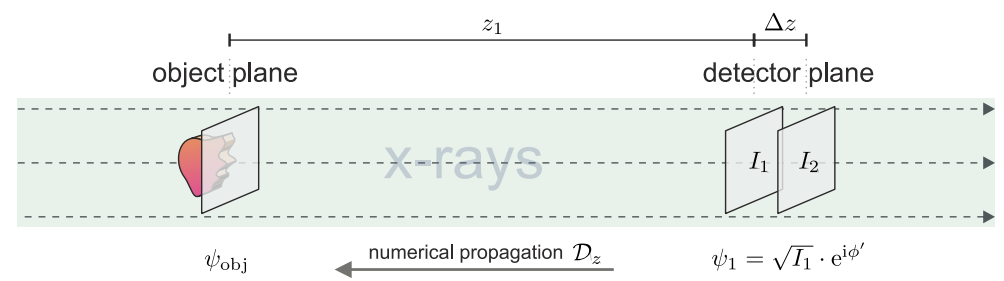

Figure 2.5: Scheme of the Holo-TIE reconstruction: Two intensity distributions $I_{1}$ and $I_{2}$, separated by a distance $\Delta z$ can be used to obtain a phase distribution $\phi^{\prime}$ by solving Eq. (2.39). With this phase and one of the measured intensities, a wave function $\psi_{1}$ is built that can be numerically propagated to the object plane, where phase and amplitude of the resulting complex wave-function correspond to the object's phase and absorption.

This equation states that a phase distribution $\phi\left(\mathbf{r}_{\perp}, z\right)$ at some distance $z$ can be obtained if the differential quotient $\partial I\left(\mathbf{r}_{\perp}, z\right) / \partial z$ and the intensity $I\left(\mathbf{r}_{\perp}, z\right)$ at this position are given.

The easiest way to approximate the differential quotient is to use the difference of two measured intensity distributions $\Delta I=I_{2}-I_{1}$, separated by a small distance $\Delta z=z_{2}-z_{1}$. This will yield an approximate phase distribution $\phi^{\prime}$ in the detector plane, which can be calculated using the Fourier implementation of the pseudo differential operators

$$
\phi^{\prime}\left(\mathbf{r}_{\perp}\right)=-\frac{k}{\Delta z} \mathcal{F}_{\perp}^{-1}\left[\frac{\boldsymbol{\nu}_{\perp}}{\left|\boldsymbol{\nu}_{\perp}\right|^{2}} \cdot \mathcal{F}_{\perp}\left\{\frac{1}{I_{1}\left(\mathbf{r}_{\perp}\right)} \mathcal{F}_{\perp}^{-1}\left(\frac{\mathcal{F}_{\perp}\left(\Delta I\left(\mathbf{r}_{\perp}\right)\right)}{\left|\boldsymbol{\nu}_{\perp}\right|^{2}} \boldsymbol{\nu}_{\perp}\right)\right\}\right]
$$

where the gradient operator and the divergence are written as multiplications in Fourier space. ${ }^{2}$ For noise free data, the zero frequency $\boldsymbol{\nu}_{\perp}=\mathbf{0}$ will be ignored. Using this approximate phase $\phi^{\prime}$ together with one of the intensities, the wave function can be approximated by

$$
\psi_{1}\left(\mathbf{r}_{\perp}, z_{1}\right)=\sqrt{I_{1}\left(\mathbf{r}_{\perp}\right)} \cdot \mathrm{e}^{\mathrm{i} \phi^{\prime}\left(\mathbf{r}_{\perp}\right)} .
$$

We assume that the phase is reconstructed in a detector plane with distance $z_{1}$ to the object plane. Thus, considering a plane wave illumination $P\left(\mathbf{r}_{\perp}\right)=1$ and using the Fresnel propagator (Eq. (1.24)) will yield the object transmission

\footnotetext{
${ }^{2}$ In practice, the term in curly brackets in Eq. (2.40) has to be treated as vector with $\mathrm{x}$ - and y- component. The subsequent multiplication in Fourier space is a scalar product that again yields a scalar quantity for $\phi^{\prime}$.
} 

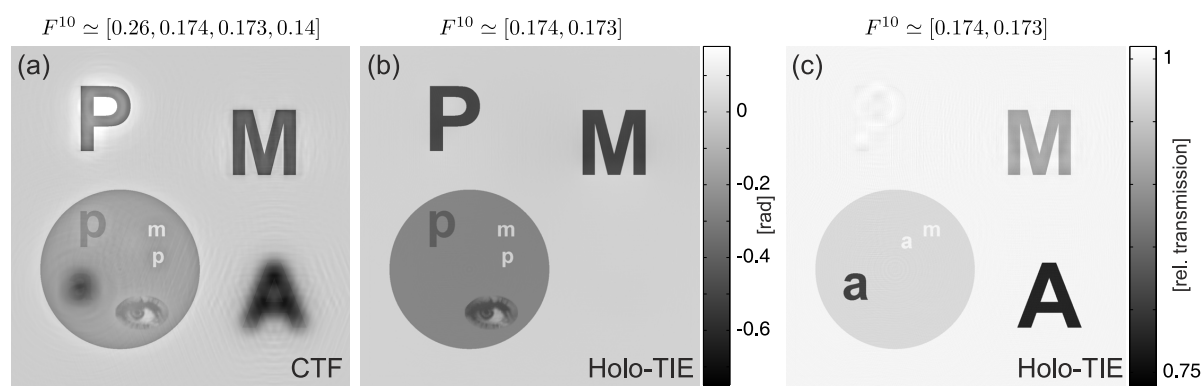

Figure 2.6: Phase retrieval for simulated, noise-free intensities of an inhomogeneous object. (a) phase distribution obtained using the CTF-based approach with the assumption of a homogeneous object. (b) Phase distribution obtained with the Holo-TIE approach, in which none of the absorbing structures is seen anymore. (c) Amplitude distribution obtained by the Holo-TIE approach, showing minimal artifacts at the position of pure-phase objects.

function. The whole reconstruction will be called Holo-TIE in the following [88] and can be formally written as

$$
O\left(\mathbf{r}_{\perp}\right)=\mathcal{D}_{-z_{1}}\left(\psi_{1}\left(\mathbf{r}_{\perp}, z_{1}\right)\right)
$$

Figure 2.5 shows a sketch of the geometric situation. This reconstruction is similar to the holographic reconstruction, with the difference that a second intensity measurement is used to reconstruct the phase distribution, which helps to eliminate the twin image problem. Importantly, no restrictive assumptions to the objects like weak absorption or phase shift are made. Like every other equation in the paraxial framework, Eq. (2.39) can be written in order to only depend on the Fresnel number. To this end, the pixel size $p$ will be separated from $\boldsymbol{\nu}_{\perp}$, so that the total prefactor reads

$$
k \frac{p^{2}}{\Delta z}=\frac{2 \pi}{\frac{\lambda z_{1}}{p^{2}}-\frac{\lambda z_{2}}{p^{2}}}=\frac{2 \pi}{\frac{1}{F_{x, 1}^{1}}-\frac{1}{F_{x, 2}^{1}}},
$$

which analogously holds for $F_{y}^{1}$.

Figure 2.6 shows the application of the Holo-TIE algorithm to simulated, noisefree data in comparison to the CTF reconstruction of an inhomogeneous object. The parameters of the object are the same as used for Fig. 1.6 and 2.3. For the CTF reconstruction, $\kappa=5$ is chosen, based on visual inspection of the mixed objects. For the high frequencies we choose $\alpha_{2}=10^{-16}$, as again four optimal dis- 
tances, obtained with $j \in[100,149,150,190]$, are used $(p=1 \mu \mathrm{m})$ to simulate the intensities. For the Holo-TIE reconstruction, only the middle two intensities with $j \in[149,150]$ are used to calculate $\Delta I=I_{2}-I_{1}$. Thus, if the difference quotient is calculated with these two intensities, the approximation to the differential quotient will be fulfilled well enough. To build the wave function $\psi_{2}=\sqrt{I_{2}} \cdot \mathrm{e}^{\mathrm{i} \phi^{\prime}}$, the second intensity is used and for back propagation, the Fresnel number $F^{10} \simeq 0.173$ is modified by a factor of 1.0016. This factor is chosen based on visual inspection such that no residual absorption effects are visible in the phase map, accounting for the fact that the detector phase distribution does not exactly yield the correct values for the plane of $I_{2}$. The CTF reconstruction (Fig. 2.6(a)) shows strong artifacts around the purely absorbing and the pure phase objects, whereas with the Holo-TIE reconstruction, shown in Fig. 2.6(b) and (c), amplitude and phase of the wave are obtained with hardly any artifacts.

\subsubsection{Treatment of noisy data}

The reconstruction in Fig. 2.6 was performed using simulated data without any noise. To fulfill the requirement that two measured intensities $I_{1}$ and $I_{2}$ approximate the differential quotient, their relative distance $\Delta z$ has to be small enough. Propagation over a small distance $\Delta z$ will, however, only give little contrast, as can be directly seen when regarding the limiting case for weak objects, given by the CTF (cf. section 1.3.1). For noisy data, ${ }^{3}$ this will set a limit to the reconstructability of the so far presented Holo-TIE approach, as low-frequency noise is enhanced too much (cf. Fig. 2.7(a)). If the difference $\Delta z$ is larger, the influence of noise will be reduced, but high-frequency artifacts disturb the reconstruction, as the approximation of the differential is not satisfied anymore (cf. Fig. 2.7(b)). One solution for this problem is to use a small $\Delta z$ and to regularize the low spatial frequencies in the inverse Laplacians, as done before in the MBA and SMO cases

$$
\frac{1}{\left|\boldsymbol{\nu}_{\perp}\right|^{2}} \rightarrow \frac{1}{\left|\boldsymbol{\nu}_{\perp}\right|^{2}+\alpha}
$$

or with a Tikhonov regularization, present in the CTF

$$
\frac{1}{\left|\boldsymbol{\nu}_{\perp}\right|^{2}} \rightarrow \frac{\left|\boldsymbol{\nu}_{\perp}\right|^{2}}{\left|\boldsymbol{\nu}_{\perp}\right|^{4}+\alpha^{2}}
$$

3 As before, simulated noise corresponding to $4 \cdot 10^{5}$ photons per pixel is added to simulated intensities used in this section. 
with some regularization parameter $\alpha$. However, contrary to the case of SMO or CTF, this regularization has no correspondence to a physical effect, such as the existence of a homogeneous object. Hence, introducing this regularization will introduce artifacts to the reconstruction; see for example Fig. 2.7(c).

There are several methods in the literature that tried to find better estimates to the differential quotient based on the measurement in more than two image planes $[163,178]$, which can be easily obtained, e.g., in an optical microscope by automatic focus variation. In the work of Waller et al. [178] different methods are compared. One approach, which the authors found to perform best, is based on a high-dimensional polynomial fitting of each pixel value over the different distances. By using for example a 20th order fit for 40 different defocus values, the first order component of the fit can be used to estimate the desired derivative $\partial I / \partial z$. How-
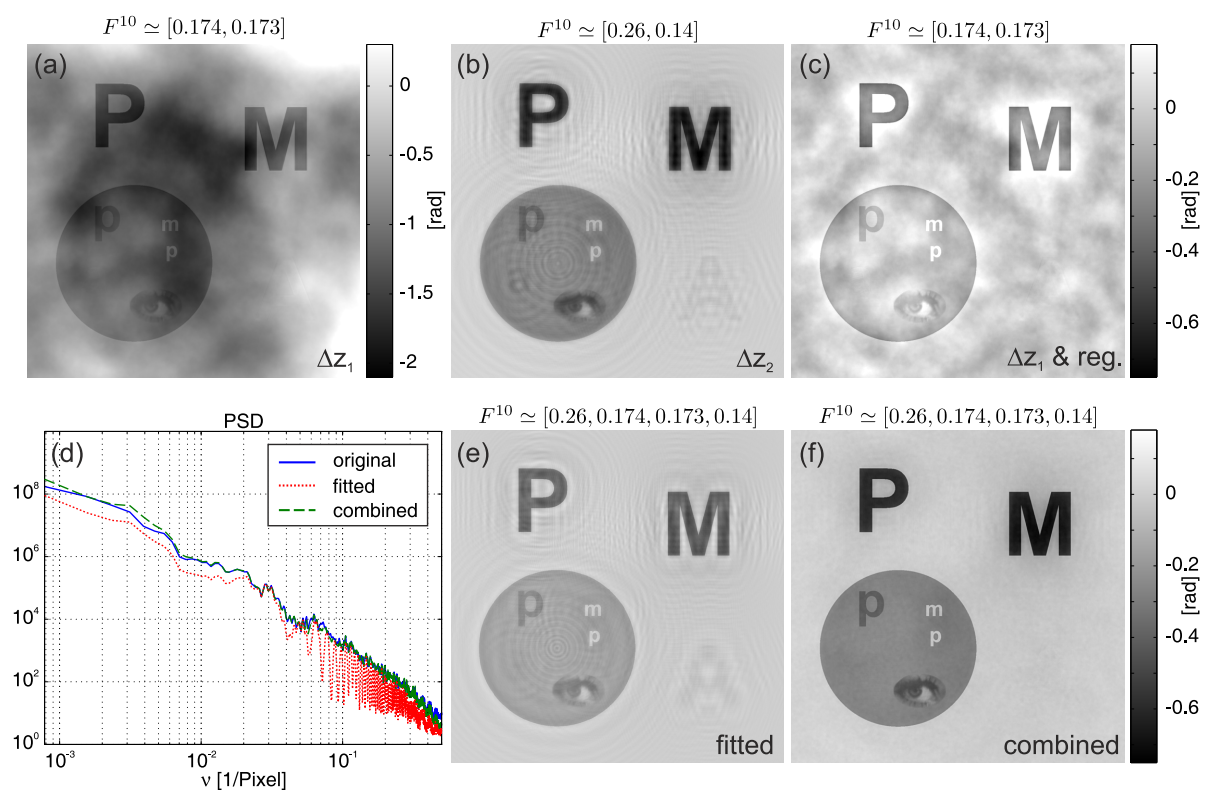

Figure 2.7: Holo-TIE phase-retrieval for simulated, noisy intensities of an inhomogeneous object. The upper row $(a, b, c)$ shows reconstructions using the two intensities, where in (a) the unregularized reconstruction of two close planes is used. (b) shows the reconstruction of two planes with a larger $\Delta z$ and (c) shows the reconstruction of the intensities used for (a) regularized with $\alpha=1 \cdot 10^{-3}$ according to Eq. (2.44). The lower row (e,f) shows reconstructions using intensities of four planes. (d) shows the azimuthally averaged PSDs for the original object and the reconstructions of (e) the first order fitting method and of (f) the Fourier-combined approach, described in the main text. 


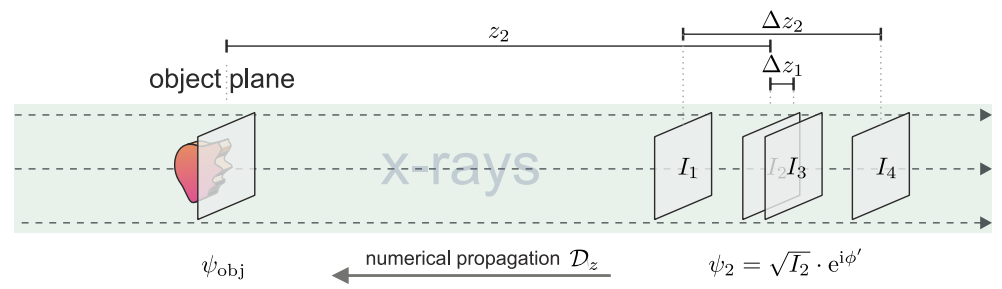

Figure 2.8: Scheme of an improved Holo-TIE reconstruction with more than two distances. Two sets of difference images, for small $\Delta z_{1}$ and for large $\Delta z_{2}$ are used. The first reconstruction will contain information about the high spatial frequencies of the detector-plane phase-distribution with spoiled low frequencies. The reconstruction for $\Delta z_{2}$ will be the other way around (see Fig. 2.7(a,b)). The idea for the improved reconstruction is to combine both to $\phi_{c o m b}^{\prime}$, to better estimate the phases in the detector plane.

ever, for x-ray measurements, the measurement at a different defocus distance means that the sample has to be physically moved to another position. This is a time consuming process and, additionally, imperfect motions or change of the sample due to drift or radiation damage have to be corrected for. Hence, a suitable reconstruction technique should allow using as little intensity measurements as possible. For a smaller number of intensity distributions, a first order fit, corresponding to the method presented in [163], turned out to yield the best results. A result of this first order fitting approach is shown in Fig. 2.7(e). For this reconstruction we used four noisy intensity distributions at Fresnel numbers that were also used in Fig. 2.6 for the CTF reconstruction. The fitting approach slightly reduces the errors at high spatial frequencies but also introduces low-frequency deviations as can be seen in the PSD in Fig. 2.7(d).

Another method that is of particular interest for holographic x-ray reconstructions can be motivated as follows: For very small propagation distances $\Delta z$, the high spatial frequencies will have better contrast than the lower ones . For larger propagation distances $\Delta z$ it is reversed. Thus, low spatial frequencies will have increased contrast but high frequencies are spoiled as the differential is not well approximated anymore (see Fig. 2.7(a,b)). Hence, the idea is to record two sets of intensities $\Delta I_{1 / 2}$ separated by $\Delta z_{1 / 2}$ as sketched in Fig. 2.8. For both sets of intensities, the phase distribution in the detector plane $\phi_{1 / 2}^{\prime}\left(\mathbf{r}_{\perp}\right)$ is obtained individually and a new phase map $\phi_{\text {comb. }}^{\prime}$ is obtained by a combination in 


\section{Fourier space}

$$
\phi_{\text {comb. }}^{\prime}\left(\boldsymbol{\nu}_{\perp}\right)=\phi_{2}^{\prime}\left(\boldsymbol{\nu}_{\perp}\right) \cdot f\left(\left|\boldsymbol{\nu}_{\perp}\right|\right)+\phi_{1}^{\prime}\left(\boldsymbol{\nu}_{\perp}\right) \cdot\left(1-f\left(\left|\boldsymbol{\nu}_{\perp}\right|\right)\right)
$$

with $f\left(\left|\boldsymbol{\nu}_{\perp}\right|\right)$ as defined in Eq. (2.31). Hence, high spatial frequencies will be fully taken from $\phi_{1}^{\prime}$ and the low frequencies from $\phi_{2}^{\prime}$ with a smooth transition at some cutoff parameter $\nu_{\text {cut }}$ and standard deviation of the transition $\sigma_{c u t}$. For the reconstruction shown in Fig. 2.7(f), we choose $\nu_{c u t}=\sigma_{c u t}=0.05$ periods $/$ pixel, based on visual inspection of the resulting phase map.

For the specific example shown in $2.7(\mathrm{f})$, the combined approach delivers the best reconstruction with minimal artifacts. However, the reconstruction quality may differ for different noise levels and choice of Fresnel numbers. The CTF-based phase-retrieval, on the other hand, has the advantage that arbitrary Fresnel numbers can be combined and good noise performance is achieved. For CTF reconstructions with the noise level used here (data not shown), no difference compared to the noise-free CTF reconstruction in Fig. 2.6 can be observed.

\subsubsection{Iterative methods}

So far we have dealt with deterministic phase-retrieval methods, i.e., a direct reconstruction formula is given. This is enabled due to the incorporation of additional constraints or by the numerical approximation of a differential quotient. As for both cases examples exist, where the algorithms fail to deliver good reconstructions, iterative methods can be used, in which an initial guess is slowly pushed to the correct solution. A major disadvantage of iterative methods is the computation speed. Depending on the kind of algorithm, even on high-end computers the reconstruction may take some minutes or more. Hence, it is a time-consuming process, especially if parameters involved in the reconstruction have to be tuned. However, if high quality reconstructions are needed and if any artifacts due to violated assumptions have to be suppressed, iterative methods are the methods of choice.

\subsubsection{Projection methods}

One approach to obtain phase information from a set of measured intensity values is the use of iterative projection algorithms. The generic idea of any iterative projection algorithm is sketched in Fig. 2.9 and works as follows: An initial guess $\psi_{0}\left(\mathbf{r}_{\perp}, 0\right)$ is propagated to the detector plane using the Fresnel propagator 
$\mathcal{D}_{z}$, yielding $\Psi_{0}\left(\mathbf{r}_{\perp}, z\right)$. We use the convention that large letters correspond to quantities in the detector plane and small letters correspond to phase, amplitude or the wave function in the object plane. Due to a measurement of the intensity $I\left(\mathbf{r}_{\perp}\right)$, the amplitudes in the detector plane are known. Hence, the current guess $\Psi_{i}$ can be updated in such a way that it fulfills the measurement

$$
\Psi_{i}^{\prime}\left(\mathbf{r}_{\perp}\right)=\sqrt{I\left(\mathbf{r}_{\perp}\right)} \cdot \frac{\Psi_{i}\left(\mathbf{r}_{\perp}\right)}{\left|\Psi_{i}\left(\mathbf{r}_{\perp}\right)\right|}
$$

This update step will be denoted as modulus constraint. The updated wave function is propagated back to the object plane, yielding a modified wave function $\psi_{i}^{\prime}$. By applying some object plane constraint

$$
\psi_{i+1}\left(\mathbf{r}_{\perp}\right)=P_{S}\left(\psi_{i}^{\prime}\left(\mathbf{r}_{\perp}\right)\right)
$$

where $P_{S}$ formally denotes a projection to this constraint, the next guess for the iteration is obtained. Typical constraints that can be employed in the object plane are, e.g., to assume a compact support of the object or to employ further knowledge about phase and amplitude. For the latter it is common to assume a homogeneous object, a purely phase shifting or a purely absorbing object or just the positivity of phase and/or absorption. If we further define the modulus projection

$$
\psi_{i}^{\prime}\left(\mathbf{r}_{\perp}\right)=P_{M}\left(\psi_{i}\left(\mathbf{r}_{\perp}\right)\right):=\mathcal{D}_{-z}\left(\sqrt{I\left(\mathbf{r}_{\perp}\right)} \cdot \frac{\mathcal{D}_{z} \psi_{i}\left(\mathbf{r}_{\perp}\right)}{\left|\mathcal{D}_{z} \psi_{i}\left(\mathbf{r}_{\perp}\right)\right|}\right),
$$

the simplest form of an iterative algorithm can be written as alternately applying the projections $P_{M}$ and $P_{S}$ to an initial guess $\psi_{0}$

$$
\psi_{i}\left(\mathbf{r}_{\perp}\right)=\left(P_{S} P_{M}\right)^{i}\left(\psi_{0}\left(\mathbf{r}_{\perp}\right)\right)
$$

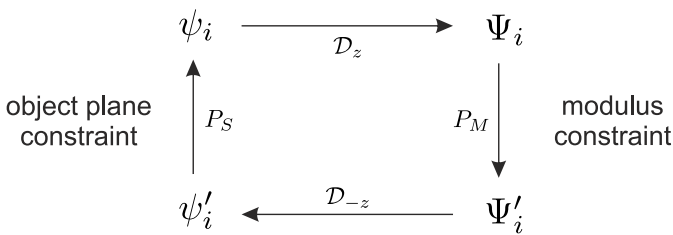

Figure 2.9: Scheme of an iterative phase-retrieval approach: A guess $\psi_{i}$ is propagated to the detector plane, where a modulus constraint is applied to yield $\Psi_{i}^{\prime}$. This updated wave function is back propagated to the object plane, where additional constraints can be applied, to yield a new guess $\psi_{i+1}$. 
known as the alternating projections algorithm (AP). Prominent examples of this algorithm are the error reduction (ER) or Gerchberg-Saxton (GS) algorithm [46]. The GS algorithm was originally proposed to reconstruct a function from the measurement of two intensities [52]. One special case of the GS algorithm is given for the assumption of pure phase objects [54]. As in this case the intensity in the object plane is known $\left(\left|\psi_{i}\left(\mathbf{r}_{\perp}\right)\right|=1\right)$, it can be enforced by a modulus constraint (Eq. (2.47)) in the object plane. In the ER algorithm, the object-plane constraint is given by enforcing a compact support of the object, i.e., for every point outside of a given support set $S$, phase and amplitude are set to zero

$$
P_{S}\left(\psi_{i}\left(\mathbf{r}_{\perp}\right)\right)= \begin{cases}\psi_{i}\left(\mathbf{r}_{\perp}\right) & \mathbf{r}_{\perp} \in S \\ 0 & \mathbf{r}_{\perp} \notin S\end{cases}
$$

The ER algorithm has the drawback that convergence is only achieved locally, so the initial guess has to be rather close to the real solution. Another very common algorithm that circumvents this limitation is the hybrid input output (HIO) algorithm [46], where the projections are not strictly enforced but a combination of the current input $\psi_{i}$ and the output $\psi_{i}^{\prime}$ is used. To this end, a relaxation parameter $\beta$ is introduced and one iteration step for the HIO implementation used in this thesis reads

$$
\psi_{i+1}=\psi_{i}-\beta \cdot P_{S} P_{M} \psi_{i}
$$

A modified HIO (mHIO) algorithm, adapted to holographic x-ray imaging, was introduced in [54], in which the update step is performed separately for phase and amplitudes. Additionally, it is enforced that the object will be a pure phase object and purely negative phase shifts are enforced. The number of iterations or data dependent measures can be used as a stopping criterion for iterative algorithms. One such criterion is the discrepancy principle used in [54]. For this purpose the residual $d$, describing the misfit of the current guess $\Psi_{i}$ in the detector plane and the measured data, is defined by

$$
d=\left.\int_{\mathbb{R}^{2}}|| \Psi_{i}\left(\mathbf{r}_{\perp}\right)\right|^{2}-\left.I\left(\mathbf{r}_{\perp}\right)\right|^{2} \mathrm{~d} \mathbf{r}_{\perp}
$$

and a noise level $D$ has to be given, such that if $d<D$ the algorithm stops. Additional stabilization of the reconstruction is achieved by using soft projections. To this end, not just the measured data is used in the modulus constraint, but a combination of the current guess in the detector plane $\Psi_{i}$ and the measured 
intensity $I$, given by

$$
I^{\dagger}\left(\mathbf{r}_{\perp}\right)=\left(1-\frac{D}{d}\right) I\left(\mathbf{r}_{\perp}\right)+\frac{D}{d}\left|\Psi_{i}\left(\mathbf{r}_{\perp}\right)\right|^{2} .
$$

If the noise level $D$ is too small, the reconstruction error $d$ will diverge. If $D$ is chosen as small as possible without noting such a divergence, good reconstructions can be obtained where zeros in the $\mathrm{CTF}$ are compensated even from a single distance measurement $[14,53]$.

The research on iterative phase-retrieval algorithms is a whole field in applied mathematics and several other algorithms exist such as Douglas-Rachford or relaxed averaged alternating reflections (RAAR) [19, 73, 102]. Another very famous iterative algorithm is ptychography $[147,170]$, where a shifting illumination in the object plane is used to obtain additional constraining information that even enables to reconstruct a non-perfect illumination [157]. Recently, ptychography was generalized to the near field [165] and for this case, also shifts along the optical axis can be used for phase retrieval [146].

The case of intensity distributions for different Fresnel numbers will be of particular interest, if comparisons to CTF-based phase-retrievals with multiple distances are desired. One of the first proposals for using multiple intensity distributions for phase retrieval was given by Allen and Oxley [2]. There are several ways to implement a multi-distance phase-retrieval. For this thesis, a very simple approach is used that is similar to the AP algorithm. Let $P_{M, i}$ be the modulus projection of the $i$-th intensity measurement with $i=1 \ldots N$. We define the algorithm, by alternately applying an AP step for every measurement plane. Thus, the update can be formulated as

$$
\psi_{i+1}=P_{S} P_{M, N}\left(\cdots\left(P_{S} P_{M, 2}\left(P_{S} P_{M, 1} \psi_{i}\right)\right)\right) .
$$

With this kind of multi-distance phase-retrieval it is easily possible to incorporate additional object plane constraints, like positivity, homogeneity or a compact support. For all iterative algorithms, the initial guess may play a crucial role if just a few numbers of iterations are carried out. One technique is to use a solution obtained with deterministic methods such as the MBA as initial guess for an iterative algorithm [63]. With these so-called hybrid approaches, already a few iterations can strongly improve the reconstruction quality [88]. Figure 2.10 shows the application of such a hybrid approach to experimental data of a Siemens-star test-pattern. Compared to the result obtained after five iterations with a uni- 

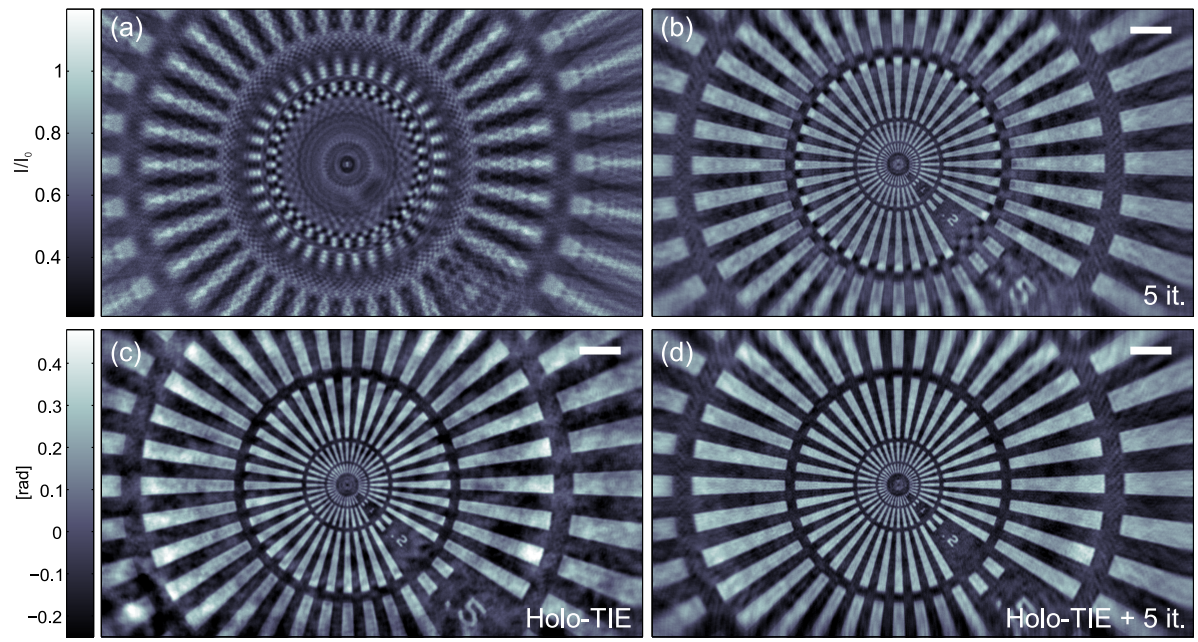

Figure 2.10: Application of a hybrid phase-retrieval approach to experimental data from a test pattern (Siemens star). (a) shows a post-processed intensity distribution obtained with an x-ray waveguide illumination for a Fresnel number of $F^{10}=0.02$. (b) shows the reconstructed phase after five iterations of an AP algorithm with homogeneity constraint, where $\psi_{0}\left(\mathbf{r}_{\perp}\right)=1$ was used as initial guess. (c) shows the regularized Holo-TIE reconstruction with two distances in which cloudy noise can be observed. (d) shows the reconstructed phase of the same algorithm used in (b) but with the Holo-TIE reconstruction as initial guess $\psi_{0}$. All scale bars denote $2 \mu \mathrm{m}$.

form initial guess (Fig. 2.10(b)), using the Holo-TIE reconstruction (Fig. 2.10(c), detailed parameters in [88]) as initial guess leads to a much better reconstructions after 5 iterations (see Fig. 2.10(d)), as the reconstructed structure now is closer to the real uniform structure of the Siemens star. Note that for the specific case of Fig. 2.10, the iterative reconstruction diverges after applying substantially more iterations, as the background still contains small residual artifacts from the imperfect illumination.

\subsubsection{Iterative reprojection phase-retrieval}

For the reconstruction of three-dimensional (3D) objects, one possible constraint can be the consistency of the object, i.e., using the fact that x-ray projections recorded at slightly different angles are not independent of each other. We refer to the next chapter, where the mathematical background of tomography is outlined and notations used in this section $\left(\mathcal{R}_{\alpha}, \mathcal{R}^{\#}, \ldots\right)$ are defined. In particular for 
compactly supported objects, Fourier slices (see Fig. 3.3) will have a finite thickness so that they overlap in the low-frequency range. As in propagation imaging high frequencies are better transferred, information from neighboring slices can be used to stabilize the low-frequency part in phase retrieval [151]. Instead of treating phase retrieval and tomography separately, a combined approach based on the multiplicative algebraic reconstruction technique (mART, see section 3.1.4) is used to automatically employ this consistency constraint.

For the following explanation we assume to have a tomographic measurement of phase-contrast images $I_{\alpha}\left(\mathbf{r}_{\perp}\right)$, i.e., intensity distribution at some propagation distance $z$ measured for many different rotation angles $\alpha$ of the object. Starting with a current guess $\psi_{\alpha, i}$ to the object wave function, the modulus constraint $P_{M}$ can be applied to yield an updated $\psi_{\alpha, i}^{\prime}$, as for the classical iterative phase-retrieval presented in the previous section. The positive phase $\phi_{\alpha, i}^{\prime}=-\arg \left(\psi_{\alpha, i}^{\prime}\right)$ and absorption $\mu_{\alpha, i}^{\prime}=-\log \left|\psi_{\alpha, i}^{\prime}\right|$ of the updated projections can be used to perform an mART-like update step (see Eq. (3.21)) by calculating update factors

$$
c_{\alpha}=\frac{\phi_{\alpha, i}^{\prime}}{\phi_{\alpha, i}}=\frac{\arg \left(P_{M} \psi_{\alpha, i}\right)}{\arg \left(\psi_{\alpha, i}\right)},
$$

that have to be applied to the $3 \mathrm{D}$ volume $f_{i}(\mathbf{r})$ using a partial back projection step $\mathcal{R}_{\alpha}^{\#}$. The updated 3D volume is then reprojected to yield new projections $\psi_{i+1}$. This method would correspond to the classical ART scheme, where the order of how projections are updated has a big influence on convergence speed. For example, taking projections separated by nearly $90^{\circ}$ will change the $3 \mathrm{D}$ volume much faster than taking neighboring projections. Another way of performing the reconstruction, is to first calculate all update factors $c_{\alpha}$ and to subsequently do the full back projection $\mathcal{R}^{\#}$ to obtain 3D update factors that can be applied to yield a new guess for the volume $f_{i}(\mathbf{r})$. This reconstruction corresponds to averaged ART and thus will be less prone to noise but also the convergence speed will be slower. It is known that averaged ART methods converge to the least-squares solution for the case of inconsistent data [30]. Hence, this kind of reconstruction is especially useful in an iterative phase-retrieval algorithm, as the current iterate may still have inconsistent 3D information. If a sequential updating is used as in classical ART, the algorithm may easily diverge. Additionally, a special kind of positivity constraint is enforced for the present implementation by back projecting the modulus of the update factors defined by Eq. (2.56). Thus, the total update 
step can be formally written as

$$
\arg \left(\psi_{\alpha, i+1}\right)=-\mathcal{R}_{\alpha}\left(\arg \left(f_{i}(\mathbf{r})\right) \cdot \mathcal{R}^{\#}\left|\frac{\arg \left(P_{M} \psi_{\alpha, i}\right)}{\arg \left(\psi_{\alpha, i}\right)}\right|\right),
$$

which analogously holds for the absorption $\mu_{\alpha, i}$. The algorithm defined by Eq. (2.57) will be termed iterative reprojection phase-retrieval (IRP) [151]. In most cases, one is interested in the reconstructed 3D volume $f(\mathbf{r})$ rather than in the projected exit wave $\psi_{\alpha}$, which in the limit or many iterations is determined by Eq. (2.57), too.

\subsubsection{Iterative Newton methods}

The phase problem in holographic x-ray imaging can be regarded as solving the nonlinear problem, given by

$$
F(\psi)=\left|\mathcal{D}_{z}(\psi)\right|^{2}=g
$$

Thus, the operator $F$ describes the image formation by free-space propagation and taking the modulus squared. If suitable a priori information is given, it could be implemented in $F$ as well. Generally the problem is ill-posed and measurements can be described by

$$
g^{\delta}=g+\delta
$$

where $\delta$ describes some random noise. Hence, the real value $g$ exists but cannot be accessed. One technique to solve nonlinear problems is the Newton method, which is demonstrated for an illustrative 1D case in Fig. 2.11. For a given initial guess $x_{0}$, a linearized version of the problem is constructed using the function $f$ and its first derivative $f^{\prime}$. If the linear problem is solved, a new guess can be obtained that is closer to the real solution $x^{\dagger}$. For problems that are close to being linear, this method will show very good convergence results. A linearized version of the Fresnel propagation is the CTF and reconstructions based on it often yield results close to the real solution, but spoiled with artifacts if the assumptions are not fully met. Hence, using Newton methods provides a promising approach that will enable reconstructions better than the CTF without enforcing further constraints [103].

For a given operator $F: D \rightarrow Y$, with $D \subset X$ being the domain on which $F$ is Frechét differentiable and $X, Y$ being Hilbert spaces, a reconstruction method can be defined by the following minimization problem, known as the iterative 


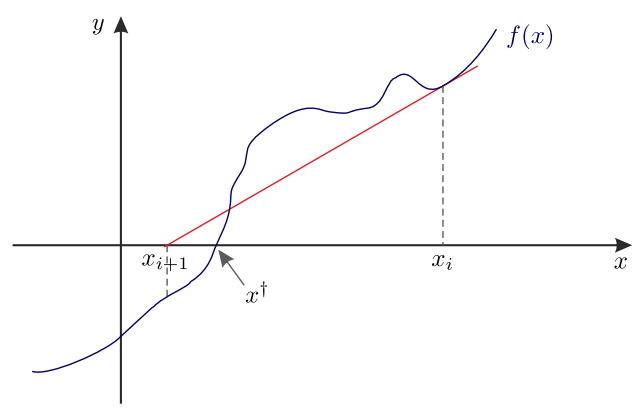

Figure 2.11: Idea of a Newton method to solve nonlinear problems $f(x)=y$ : At some guess $x_{i}$ the nonlinear problem is linearized (red line) and the linear problem is solved instead to yield a new guess $x_{i+1}$ being closer to the real solution. For suitable starting points, this approach will converge to the real solution $x^{\dagger}$.

Gauss-Newton method (IRGN) $[9,74]$

$$
\min _{h_{i} \in X}\left(\left\|F\left(\psi_{i}\right)+F^{\prime}\left(\psi_{i}\right) h_{i}-g\right\|+\alpha_{i} R\left(h_{i}\right)\right) .
$$

Here, $F^{\prime}$ is the Frechét derivative of $F, R\left(h_{i}\right)$ is some penalty term, $\alpha_{i}$ a regularization parameter and $\|\cdot\|$ denotes a norm in $X$ or $Y$. In each Newton step, a convex minimization step has to be performed, which can be done with a conjugate gradient (CG) approach [47]. The new guess is calculated by

$$
\psi_{i+1}=\psi_{i}+h_{i}
$$

The penalty term is often chosen to $R\left(h_{i}\right)=\left\|\psi_{i}+h_{i}-\psi_{0}\right\|$, which minimizes the difference to the initial guess $\psi_{0}$ and therefore has an additional regularizing effect [74]. Consider a single measurement for a given Fresnel number, which is described by $F_{j}(\psi)=g_{j}$. To incorporate multiple measurements for different Fresnel numbers, a new operator $F_{\text {total }}=\left(F_{1}, \ldots, F_{j}, \ldots, F_{N}\right)$ can be defined as the tuple of all single operators. Here, $N$ denotes the number of imaging planes. The new problem, which has to be solved is thus given by [103]

$$
F_{\text {total }}(\psi)=g_{\text {total }}=\left(g_{1}, \ldots, g_{j}, \ldots, g_{N}\right)
$$

The operator defined by this equation is component-wise differentiable, so that the total Frechét derivative $F_{\text {total }}^{\prime}$ is the tuple of the single derivatives $F_{j}^{\prime}$. Additional projection constraints $P_{S}$ (e.g. support projection) can be implemented 
by constructing a new operator $F_{\text {total }}=P_{S} F$, with $F_{\text {total }}^{\prime}=P_{S} F^{\prime}$, as projections are linear operators. To further include a (nonlinear) positivity constraint, the following expression is added as additional regularization to the expression in brackets in Eq. (2.60) [103]:

$$
\gamma\left\|\min \left(\psi_{i}, 0\right)+\Theta\left(-\psi_{i}\right) \cdot h_{i}\right\|^{2} \quad \Theta\left(-\psi_{i}\right)(x)= \begin{cases}1 & \text { for } \psi_{i}(x)<0 \\ 0 & \text { else }\end{cases}
$$

This term only has an influence for negative values of $\psi_{i}$ and penalizes them. Therefore, the algorithm will prefer positive solutions. The parameter $\gamma$ can be used to counterbalance this penalty term to other regularizations.

In this work, a stopping rule based on the residual reduction $\Delta d=d_{i}-d_{i-1}$ with $d_{i}$ as defined in Eq. (2.53) is chosen. Additional constraints can be incorporated by choosing a special norm, e.g., $\|\cdot\|^{1}$ to enforce total variation minimization or by changing the penalty term $R\left(h_{i}\right)$, e.g., such that the difference to another wave function $\psi^{\dagger}$, which could be for example a time frame before the current reconstruction, is minimized. Both approaches are very successfully applied, e.g., for magnetic resonance imaging [22], where real time imaging is enabled by reconstructing only a few Fourier slices [173].

Figure 2.12 shows the application of three different iterative, multi-distance phaseretrieval algorithms (AP, mHIO and IRGN) to simulated data. Four noisy intensity distributions for Fresnel numbers as in Fig. 2.7(e) and (f) were used for each algorithm. The reconstructed amplitude and phase, obtained with the mHIO reconstruction, are shown in Fig. 2.12 (b) and (e). Convergence of the algorithm is ensured by using soft projections (Eq. (2.54)) with $D=10^{-3}$ and it was stopped after 1000 iterations, as for this iteration number the residual $d$ was still larger than $D$. A limiting support was manually determined, shown as a dotted line, and the relaxation parameters were $\beta=0.8$ for both amplitudes and phases (see Eq. (2.52)). Additionally, positivity of $\delta\left(\mathbf{r}_{\perp}\right)$ and $\beta\left(\mathbf{r}_{\perp}\right)$ is enforced by only allowing a maximum amplitude of 1 and a maximum phase shift $\phi$ of 0 . The whole reconstruction takes about 5 minutes on a state of the art personal computer. All iterative algorithms presented here are implemented in Matlab and to increase the convergence speed, the graphics processing unit (GPU) is used for calculations, which speeds up the algorithms by a factor of about 10. For the reconstruction with the AP algorithm, shown in Fig. 2.7(a) and (d), hard projections are used and it stops again after 1000 iterations, which are reached in about 2.5 minutes on the same computer. No support constraint was enforced but the positivity of 

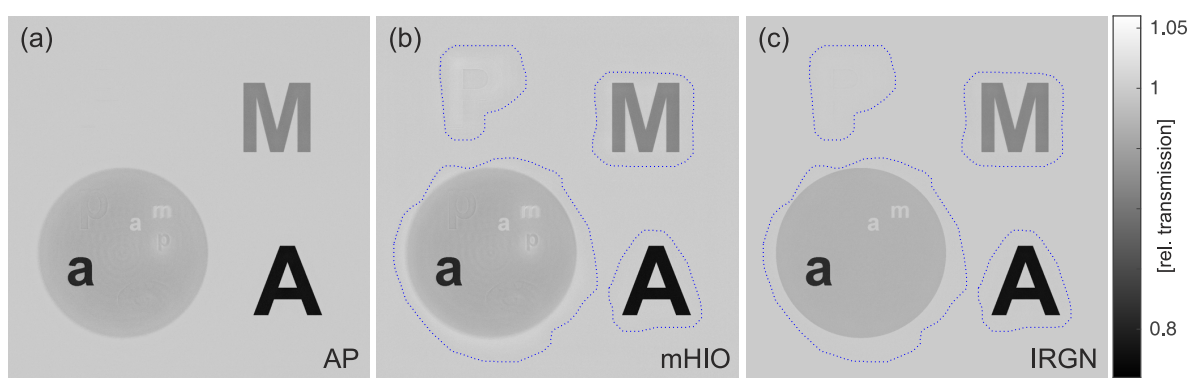

(d)

(e)

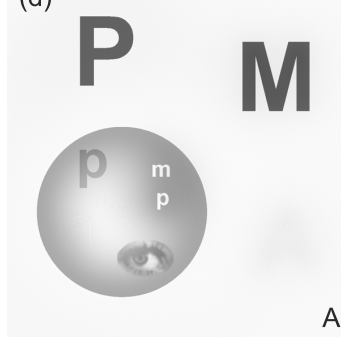

AP

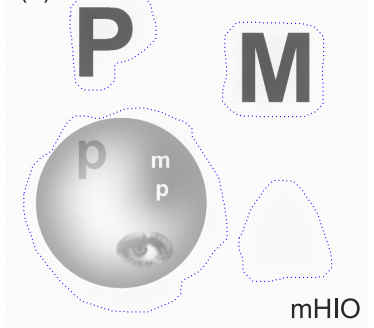

(f)

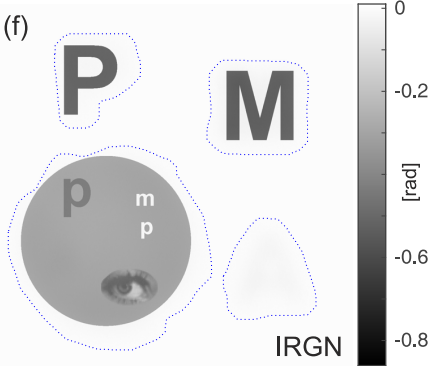

Figure 2.12: Application of iterative, multi-distance phase-retrieval for reconstructing an inhomogeneous object for the four Fresnel numbers also used in Fig. 2.7(e,f). (a,b,c) show the reconstructed amplitude and (d,e,f) the reconstructed phase distribution obtained with (a,d) an AP algorithm with positivity constraint after 1000 iterations, (b,e) an $\mathrm{mHIO}$ algorithm with positivity and support constraint after 1000 iterations and with (c,f) the IRGN method with positivity constraint, where for the latter a residual reduction of $10^{-4}$ was chosen as stopping criterion. For the $\mathrm{mHIO}$ reconstruction, the support was manually chosen and is depicted as dotted lines.

$\delta\left(\mathbf{r}_{\perp}\right)$ and $\beta\left(\mathbf{r}_{\perp}\right)$ was ensured as for the mHIO reconstruction. The IRGN method, results by which are shown in 2.7 (c) and (f), was implemented by the group of Thorsten Hohage of the Institute for Numerical and Applied Mathematics with special efforts from Simon Maretzke. The algorithm is stopped after a residual reduction of $\Delta d=10^{-4}$, which is reached after 26 Newton iterations with a total of 2239 CG iterations. The total GPU-based reconstruction takes about 13 minutes on the same computer as used before. The regularization parameter $\alpha$ is estimated from the data and iteratively decreased. Support and positivity constraints are employed as described earlier. The weighting factor for the positivity is chosen to $\gamma=0.3 \alpha_{0}$, where $\alpha_{0}$ is the data-dependent first choice of the regularization parameter $\alpha$. Further details about the actual implementation can be found in [103]. 
For single-distance phase-retrieval, the use of support information in the mHIO algorithm can significantly improve the reconstruction, especially in the case of very noisy data [16]. However, although the additional knowledge about the support is present, no significant improvement compared to the AP reconstruction can be observed in the multi-distance phase-retrieval shown in Fig. 2.12. This may be explained by the fact that due to the multiple distances, sufficient information is present to ensure convergence to the right solution in these similar reconstruction methods. Note that both algorithms failed to reconstruct the very low frequencies that experienced only little contrast transfer. This effect can be reduced by increasing the number of iterations, but even after 100000 iterations, the AP reconstruction shows low-frequency artifacts. By contrast, the reconstruction obtained with the IRGN method shows a nearly perfect match, in which no difference to the real object is observable anymore. If the Newton method is carried out without support information, a slight low frequency variation remains visible but the reconstruction of the large circle shows less artifacts than those achieved with the projection methods. 


\section{Computed tomography}

In the framework of the projection approximation, presented in chapter 1, x-ray images will contain information about the projected refractive index, namely about the absorption $\mu\left(\mathbf{r}_{\perp}\right)$ and with suitable phase retrieval, also about the phase $\phi\left(\mathbf{r}_{\perp}\right)$. An x-ray image will show information about internal structures that can stem from different positions along the optical axis in a 3D object. Computed tomography $(\mathrm{CT})$ is an imaging technique that allows the reconstruction of the internal 3D distribution of the underlying constants by measuring projections under different rotational angles $\alpha$. Regarding the absorption and phase shift of an object, as given by Eq. (1.61) and $(1.62)^{1}$

$$
\begin{aligned}
& \bar{\delta}(y, z)=-\frac{\phi(y, z)}{k}=\int_{-d}^{0} \delta(\mathbf{r}) \mathrm{d} x, \\
& \bar{\beta}(y, z)=\frac{\mu(y, z)}{2 k}=\int_{-d}^{0} \beta(\mathbf{r}) \mathrm{d} x,
\end{aligned}
$$

the problem reduces to the reconstruction of a function, given its integrated values along a line. The following sections addresses the mathematical framework that enables such a reconstruction, followed by an assessment of typical artifacts that may occur.

\subsection{Mathematical background}

\subsubsection{Radon transform}

The fundamental mathematics of reconstructing a function, when its integral values are given, was derived by Johann Radon in 1917 [143]. Consider the geometric situation sketched in Fig. 3.1: We have a function $f(x, y)$, representing an object by mapping each point $\mathbf{r}_{\perp} \in \mathbb{R}^{2}$ to a value $g \in \mathbb{R}$ (e.g., $\delta$ or $\beta$ of the refractive

\footnotetext{
${ }^{1}$ Note that the choice of $x, y$ differs from the convention used in chapter 1 and 2. Now, $z$ denotes the vertical axis, which is oriented parallel to the rotation axis. In the following, we will choose the $x$-axis to be the optical axis for propagation.
} 


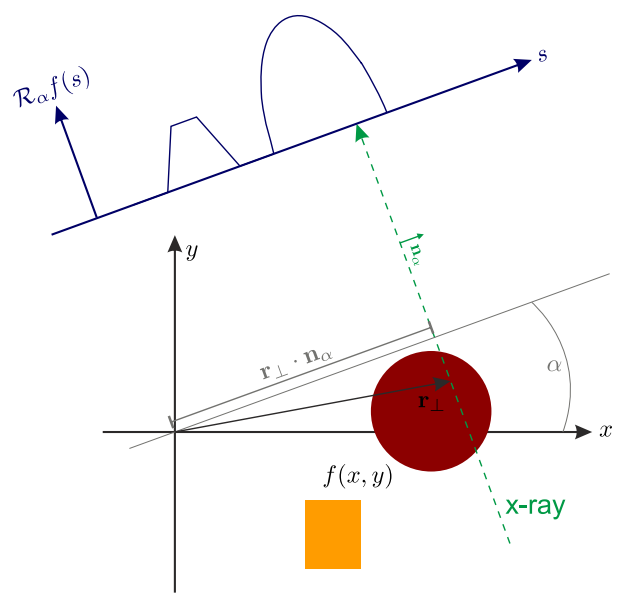

Figure 3.1: Illustration of the Radon transform. A $2 D$ object described by a function $f(x, y)$ is illuminated by parallel x-rays (dashed green line) forming an angle $\alpha$ with the $y$-axis. After interaction with the object, the projected values form a one-dimensional profile $\mathcal{R}_{\alpha} f(s)$ in dependence on the position $s$ along a line, perpendicular to the projection direction.

index). In the example given in Fig. 3.1 the object is chosen to consist of regions of constant values, forming a sphere with $f(x, y)=1$ and a rectangle with $f(x, y)=0.8$, indicated by the different colors. Upon illumination with x-rays under an angle $\alpha$ (the dashed green arrow), the wave weakly interacts with each point $(x, y)$ and is attenuated and/or the phase shift is changed. These values will accumulate over the whole distance, so that behind the object a projection of all the values can be measured. For a given angle $\alpha$ this will be termed the (2D) Radon transform $\mathcal{R}_{\alpha} f$. Mathematically, it can be expressed as [29]

$$
\mathcal{R}_{\alpha} f(s):=\int_{\mathbb{R}^{2}} f\left(\mathbf{r}_{\perp}\right) \delta\left(\mathbf{r}_{\perp} \cdot \mathbf{n}_{\alpha}-s\right) \mathrm{d} \mathbf{r}_{\perp},
$$

where $\mathbf{r}_{\perp}=(x, y)^{T}$ defines the coordinate vector in the image plane (perpendicular to the rotation axis), $s$ is the coordinate of the projected profile, $\delta\left(s-s_{0}\right)$ denotes the one-dimensional Dirac delta distribution and $\mathbf{n}_{\alpha}=(\cos \alpha, \sin \alpha)^{T}$ is the normal vector, perpendicular to the projection direction. The scalar product $\mathbf{r}_{\perp} \cdot \mathbf{n}_{\alpha}$ yields the projection of the coordinate vector $\mathbf{r}_{\perp}$ to a line, forming the angle $\alpha$ with the $x$-axis (solid, gray line in Fig. 3.1). Thus, the argument of the delta-distribution vanishes for every point forming a straight line of angle $\alpha$ with the $y$-axis with distance $s$ to the origin. This means that only points on the dashed green line will 
contribute to $\mathcal{R}_{\alpha} f(s)$, so the $2 \mathrm{D}$ integral in Eq. (3.3) is in fact a one-dimensional integral over this line. An equivalent formulation of Eq. (3.3) is often found in the literature [84], which can be derived by inserting the definitions of $\mathbf{r}_{\perp}$ and $\mathbf{n}_{\alpha}$ in Eq. (3.3), calculating the scalar product, performing a $2 \mathrm{D}$ coordinate transform and using the relation $\sin ^{2} \alpha+\cos ^{2} \alpha=1$. After simplifications, this leads to

$$
\mathcal{R}_{\alpha} f(s):=\int_{\mathbb{R}} f(s \cos \alpha-y \sin \alpha, s \sin \alpha+y \cos \alpha) \mathrm{d} y .
$$

Note that with the definition of the Radon transform given by Eq. (3.3) the generalization of the Radon transform to higher dimensions $n$ is easily possible [116]. For example, in the case of the $3 \mathrm{D}$ radon transform as a function $\mathcal{R} f$ : $\mathbb{R}^{3} \rightarrow \mathbb{R}$, in which the integral is calculated over a plane instead of a line, the vector $\mathbf{n}_{\alpha}$ will become the normal vector of this plane.

If we consider the Radon transform of a single point located at some distance to the origin, the $2 \mathrm{D}$ plot of all projected values as a function of the angle $\alpha$ will be a sine curve. As an image can be regarded as a superposition of single points, this kind of data representation is called sinogram (see Fig. 3.2(c)). The following sections will deal with reconstruction methods, to obtain $f(x, y)$ for a

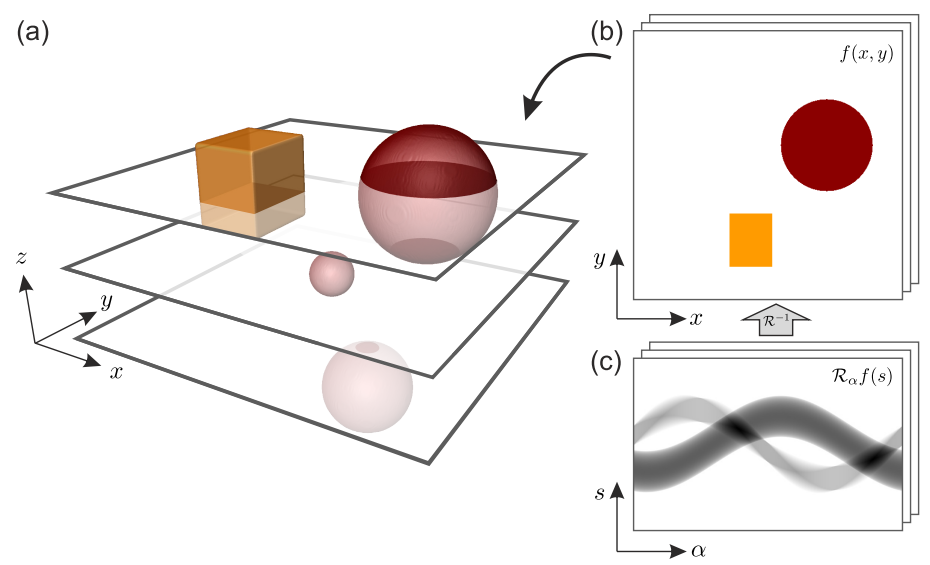

Figure 3.2: From $2 D$ slices to a $3 D$ object: In a tomographic scan, each vertical position in a projection image $(z=$ const.) forms (c) a sinogram, i.e., the projected profiles $\mathcal{R}_{\alpha} f(s)$ plotted as a function of the projection angle $\alpha$. These can be inverted to obtain (b) a reconstructed slice $f(x, y)$ that subsequently can be virtually stacked to yield (a) a $3 D$ volume. 
given sinogram $p(\alpha, s):=p_{\alpha}(s):=\mathcal{R}_{\alpha} f(s)$ that can be formally written as

$$
f(x, y)=\mathcal{R}^{-1}(p(\alpha, s)),
$$

with the inverse Radon transform $\mathcal{R}^{-1}$. In this formalism, a 2D image $f(x, y)$ can be obtained from a set of 1D projections at different angles $\alpha$. To obtain a 3D reconstruction of an object, many images $f(x, y)$ can be stacked in the $z$ direction, yielding a 3D dataset $f(x, y, z)$, as illustrated in Fig. 3.2.

\subsubsection{Fourier slice theorem}

Let us consider the $n$-dimensional Radon transform $\mathcal{R} f(s)$, of a function $f(\mathbf{r})$ with $\mathbf{r} \in \mathbb{R}^{n}$ and $\mathbf{n}$ being the normal vector of a hyperplane in $\mathbb{R}^{n-1}$. The Fourier transform ${ }^{2}$ of such a projection with respect to $s$ reads

$$
\begin{aligned}
\mathcal{F}_{s}(\mathcal{R} f)(\rho) & =\int_{\mathbb{R}}\left(\mathrm{e}^{-2 \pi \mathrm{i} \rho s} \int_{\mathbb{R}^{n}} f(\mathbf{r}) \delta(\mathbf{r} \cdot \mathbf{n}-s) \mathrm{d} \mathbf{r}\right) \mathrm{d} s \\
& =\int_{\mathbb{R}^{n}} \mathrm{e}^{-2 \pi \mathrm{i} \mathbf{r} \cdot \mathbf{n}} f(\mathbf{r}) \mathrm{d} \mathbf{r} .
\end{aligned}
$$

Here, the order of integration has been changed and the integral over $s$ was carried out, eliminating the delta distribution. If we now define $\boldsymbol{\nu}:=\rho \mathbf{n}$ as the coordinate vector in reciprocal space, Eq. (3.6) is proportional to the Fourier transform of the function $f(\mathbf{r})$ with respect to the coordinate vector $\mathbf{r}$

$$
\mathcal{F}_{s}(\mathcal{R} f)(\rho)=\int_{\mathbb{R}^{n}} \mathrm{e}^{-2 \pi \mathrm{i} \boldsymbol{\nu} \cdot \mathbf{r}} f(\mathbf{r}) \mathrm{d} \mathbf{r}=\mathcal{F}_{\mathbf{r}}(f)(\boldsymbol{\nu}) .
$$

This equation is known as the Fourier slice theorem, which proves that the Radon transform is invertible for every dimension $n$ [116]. It states that the one-dimensional Fourier transform of a projection $\mathcal{R} f(s)$ yields the values of the $n$-dimensional Fourier transform of the original function $f(\mathbf{r})$ along a slice through the origin defined by $\boldsymbol{\nu}=\rho \mathbf{n}$. Figure 3.3 illustrates this situation for the 2D case, for which the Fourier slice theorem reads

$$
\tilde{p}_{\alpha}(\rho)=\mathcal{F}_{s}\left(\mathcal{R}_{\alpha} f\right)(\rho)=\tilde{f}(\rho \cos \alpha, r \sin \alpha),
$$

where the definition of $\mathbf{n}_{\alpha}$ and the usual notation for Fourier transformed quantities were used. Hence, it follows that if all angles are measured, the Fourier space can

$\overline{2}$ We use the Fourier transform as defined in [29] (Eq. (A.4)), so that prefactors of $\sqrt{2 \pi}$ vanish. 


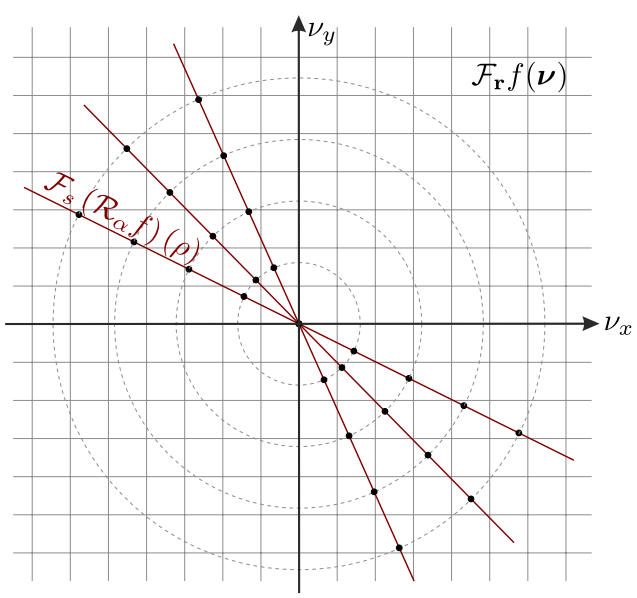

Figure 3.3: Illustration of the Fourier slice theorem for $2 D$ case $(n=2)$ : The $1 D$ Fourier transform of a projection $\mathcal{R}_{\alpha} f(s)$ corresponds to a slice (red line) in the $2 D$ Fourier transform of the object $f(\boldsymbol{\nu})$. If the function is given at discrete points, indicated by the black dots, a sophisticated interpolation in Fourier space is necessary to avoid artifacts in the tomographic reconstruction.

be completely determined and the initial function $f(\mathbf{r})$ can be reconstructed by applying an inverse Fourier transform. This reconstruction method is known as the direct Fourier method and is widely used, e.g., in magnetic resonance imaging (MRI) [22]. However, as becomes clear when the function $f(\mathbf{r})$ is given at discrete points and a finite number of angles is used, the Fourier space will not be equally sampled, as can be seen in Fig. 3.3. In particular, sample points in Fourier space will not be on a rectangular grid. Hence, if the FFT is going to be used, interpolation methods are necessary that can introduce artifacts in real space. Moreover, as the point density near the origin will be higher than for larger spatial frequencies, high resolution information in the image can be underrepresented. As a consequence one has to weight the projections in the right way, which will lead to the filtered back projection presented in the next section.

\subsubsection{Filtered back projection}

Due to the drawbacks of the direct Fourier inversion present for the case of discrete data, another method is widely used for computed tomography [29]. To derive this 
approach, we write the function $f\left(\mathbf{r}_{\perp}\right)$ as the inverse Fourier transform of $\tilde{f}$

$$
f\left(\mathbf{r}_{\perp}\right)=\int_{\mathbb{R}^{2}} \tilde{f}(\boldsymbol{\nu}) \mathrm{e}^{2 \pi \mathrm{i} \boldsymbol{\nu} \cdot \mathbf{r}_{\perp}} \mathrm{d} \boldsymbol{\nu} .
$$

Using the transform to polar coordinates $\left(\nu_{x}, \nu_{y}\right)^{T}=(\rho \cos \alpha, \rho \sin \alpha)^{T}$, with the Jacobian $\left|\partial\left(\nu_{x}, \nu_{y}\right) / \partial(\rho, a)\right|=\rho$, Eq. (3.9) reads

$$
f\left(\mathbf{r}_{\perp}\right)=\int_{0}^{2 \pi} \int_{0}^{\infty} \tilde{f}(\rho \cos \alpha, \rho \sin \alpha) \mathrm{e}^{2 \pi \mathrm{i} \rho \mathbf{r}_{\perp} \cdot \mathbf{n}_{\alpha}} \cdot \rho \mathrm{d} \rho \mathrm{d} \alpha,
$$

where $(x \cos \alpha+y \sin \alpha)=\mathbf{r}_{\perp} \cdot \mathbf{n}_{\alpha}$ has been applied to simplify the exponent. If we use a symmetry relation of the Fourier transform, the integral from 0 to $2 \pi$ can be split into two and simplified to yield [29]

$$
f\left(\mathbf{r}_{\perp}\right)=\int_{0}^{\pi} \int_{-\infty}^{\infty} \tilde{f}(\rho \cos \alpha, \rho \sin \alpha) \mathrm{e}^{2 \pi \mathrm{i} \rho \mathbf{r}_{\perp} \cdot \mathbf{n}_{\alpha}} \cdot|\rho| \mathrm{d} \rho \mathrm{d} \alpha .
$$

Next, the Fourier slice theorem can be applied to replace the function $\tilde{f}$ by its projections $\tilde{p}$

$$
f\left(\mathbf{r}_{\perp}\right)=\int_{0}^{\pi} \int_{-\infty}^{\infty} \tilde{p}_{\alpha}(\rho) \mathrm{e}^{2 \pi \mathrm{i} \rho \mathbf{r}_{\perp} \cdot \mathbf{n}_{\alpha}} \cdot|\rho| \mathrm{d} \rho \mathrm{d} \alpha
$$

Before we discuss the meaning of Eq. (3.12), let us first look at some definitions: For a given $1 \mathrm{D}$ projection $p_{\alpha}(s)$ we define the partial back projection $\mathcal{R}_{\alpha}^{\#}$ as

$$
\left(\mathcal{R}_{\alpha}^{\#} g\right)(\mathbf{r})=p_{a}\left(\mathbf{r} \cdot \mathbf{n}_{\alpha}\right)=p_{\alpha}(x \cos \alpha+y \sin \alpha)
$$

Hence, each point $\mathbf{r} \in \mathbb{R}^{2}$ is mapped to the value $p(s)$, where $s$ is determined via the projection of the coordinate vector $\mathbf{r}$ in the direction of the normal vector $\mathbf{n}_{\alpha}$ by the scalar product. In other words, the values of $p(s)$ are smeared out to the $2 \mathrm{D}$ space, such that each point $\mathbf{r}$ obtains the value, given in the projection at coordinate $s$, where the point $\mathbf{r}$ would be projected to during a Radon transform. We also define the (full) back projection $\mathcal{R}^{\#}$ of a projection $p_{\alpha}(s)$ via

$$
\left(\mathcal{R}^{\#} g\right)(\mathbf{r}):=\int_{0}^{\pi} p_{\alpha}\left(\mathbf{r} \cdot \mathbf{n}_{\alpha}\right) \mathrm{d} \alpha
$$


which performs the partial back projection for every angle $\alpha$. We further define a filtered projection $p_{\alpha}^{F}$ by applying a filter to the projections $p_{\alpha}$ in Fourier space

$$
p_{\alpha}^{F}(s):=\mathcal{F}^{-1}\left(\tilde{h}(\rho) \tilde{p}_{\alpha}(\rho)\right)=\int_{-\infty}^{\infty} \mathrm{e}^{2 \pi \mathrm{i} \rho s} \tilde{h}(\rho) \tilde{p}_{\alpha}(\rho) \mathrm{d} \rho
$$

with a filter kernel $\tilde{h}(\rho)$. Examination of Eq. (3.12) reveals that it can be rewritten using filtered projections with the kernel $\tilde{h}(\rho)=|\rho|$. Hence, it reads

$$
f(\mathbf{r})=\int_{0}^{\pi} p_{\alpha}^{F}\left(\mathbf{r} \cdot \mathbf{n}_{\alpha}\right) \mathrm{d} \alpha
$$

which is the back projection of the filtered projections, motivating the name filtered back projection. The reconstruction can also be formally written as

$$
f(\mathbf{r})=\mathcal{R}^{\#}\left(p_{\alpha}^{F}(s)\right)=\mathcal{R}^{\#}\left(\left(\mathcal{R}_{\alpha} f\right) * h\right)(\mathbf{r}),
$$

where $h(s)=\mathcal{F}^{-1}(\tilde{h}(q))$ and * denotes the convolution. The filter $\tilde{h}(q)=|q|$, known as the ramp or Ram-Lak filter [144], acts as a high pass to the projections. To suppress noise enhancement at high spatial frequencies the filter can be combined with a window function (e.g., Shepp-Logan [159], Hamming, Blackman or others [29]), leading to a different functional behavior at high spatial frequencies. However, for projections obtained from phase-retrieval algorithms, high-frequency noise is typically not as pronounced as in the classical case for absorption data, such the standard ramp filter is sufficient in most cases. For this work, the implementation of the filtered back-projection in Matlab is used.

\subsubsection{Algebraic reconstruction technique}

In the previous sections, tomographic reconstructions were derived for the analytical case. Now we want to consider the case where the function $f(\mathbf{r})$ and its projections $p_{\alpha}(s)$ are given on a discrete grid, e.g., as an image represented by pixels. As the Radon transform defines a linear operator, it can be represented by a system matrix $R$. Let $\alpha_{i}$ be the discretization of the angle $\alpha$ with $i \in\left\{1,2, \ldots, N_{\alpha}\right\}$. A discretization of an image $f(\mathbf{r})$ with $m=N_{x} \times N_{y}$ pixels is given by $\mathbf{f} \in \mathbb{R}^{m}$. Similarly the discretization of $p_{\alpha_{i}}(s)$ with $n$ sampling points can be written as $\mathbf{p}_{i} \in \mathbb{R}^{n}$. Thus the system matrix $R_{i}$ for projection under an angle $\alpha_{i}$ will be an $m \times n$ matrix and the Radon transform can be formally written as a linear system 


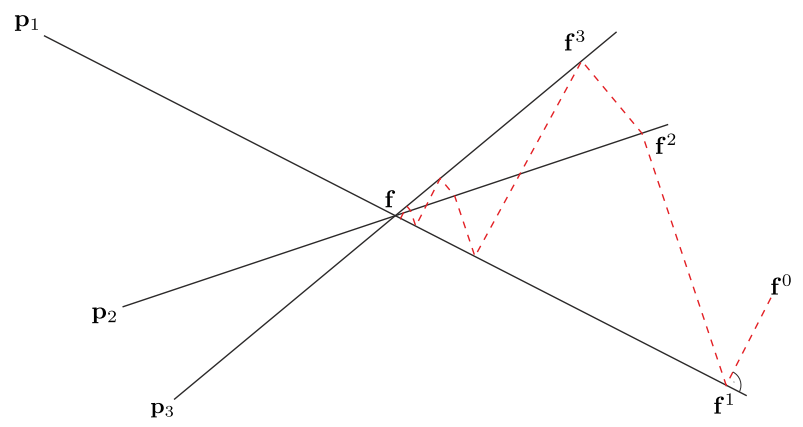

Figure 3.4: Illustration of the Kaczmarz method in $\mathbb{R}^{2}$. The measurements $\mathbf{p}_{i}$ are given as three lines that have the desired solution $\mathbf{f}$ as a common intersection. Starting with an arbitrary solution $\mathbf{f}_{0}$ and projecting it to the measurement $\mathbf{p}_{1}$ brings the initial guess to $\mathbf{f}^{1}$, which is closer to the real solution $\mathbf{f}$. By applying successive orthogonal projections $\mathbf{p}_{i}$, the method will converge to the solution f.

of equations [116]

$$
\mathbf{p}^{i}=R^{i} \mathbf{f}
$$

defined for each angle $\alpha_{i}$. For $\mathbf{p}=\left(\mathbf{p}^{1}, \ldots, \mathbf{p}^{N_{\alpha}}\right)$ and $R=\left(R^{1}, \ldots, R^{N_{\alpha}}\right)$ this can be written as a single operation

$$
\mathbf{p}=R \mathbf{f}
$$

In principle this system of equations could be directly solved. However, for typical dimensions of an image with $N_{x}=N_{y}=1000$ pixels, $n=1000$ points in the projection and $N_{\alpha}=1000$ angles, the system matrix $R$ has $10^{12}$ entries, hence a direct inversion is not easily possible. Instead, iterative methods can be used to solve the equations, as proposed by Kaczmarz [83]. In practice the methods work by alternately applying orthogonal projections $P_{i}^{3}$ onto the affine subspaces defined by the projection data $\mathbf{p}^{i}$ [116]. In other words, each measurement $\mathbf{p}^{i}$ is consecutively enforced by projecting the current guess to this measurement, similar to iterative phase-retrieval. If the measurements $\mathbf{p}^{i}$ have a consistent solution, it can be shown that this method will converge to a solution $\mathbf{f}$ of Eq. (3.18) [116].

Figure 3.4 illustrates the algorithm for $\mathbf{f} \in \mathbb{R}^{2}$ and $\mathbf{p}_{i} \in \mathbb{R}$. Under certain assumptions, the current guess $\mathbf{f}^{k}$ will converge to the solution $\mathbf{f}$ after successively applying orthogonal projections to an initial guess $\mathbf{f}^{0}[116]$. For $\mathbf{r}_{j}=\left(R_{j 1}, R_{j 2}, \ldots, R_{j m}\right)^{T}$

${ }^{3}$ Note that the projection $P_{i}$ is meant in a mathematical way and not to be confused with the measured projection data $\mathbf{p}^{i}$. 
being the transposed row $j$ of the matrix $R$, one iteration step can be written as $[84]$

$$
\mathbf{f}^{k+1}=\mathbf{f}^{k}+\frac{\left(p_{j}-\mathbf{r}_{j} \cdot \mathbf{f}^{k}\right)}{\left|\mathbf{r}_{j}\right|^{2}} \mathbf{r}_{j} .
$$

According to the above equation, points along a projection line are corrected by the normalized difference of the reprojected value of a current guess, given by $\mathbf{r}_{j} \cdot \mathbf{f}^{k}$, and the measured value of the projection $p_{j}$ along this line. The application of the Kaczmarz method to solve a tomographic problem, known as the (additive) algebraic reconstruction technique (ART), was first suggested by Gordon et al. [56]. In the same paper they also suggested a multiplicative algebraic reconstruction technique (mART), in which the iteration step instead is multiplicative

$$
\mathbf{f}^{k+1}=\mathbf{f}^{k} \frac{p_{j}}{\mathbf{r}_{j} \cdot \mathbf{f}^{k}}
$$

Here, the current guess $\mathbf{f}^{k+1}$ is updated via a multiplication with the quotient of the measured projection $p_{j}$ and the reprojection of the previous guess $\mathbf{r}_{j} \cdot \mathbf{f}^{k}$. Several other variants exist that differ in the calculation of the next step, e.g., by using averaged projections [5]. It has been shown that averaged projections, i.e., first calculating update values for every projection $\mathbf{p}^{i}$, averaging them and applying the averaged update to yield the next guess $\mathbf{f}^{k+1}$, lead to the least-squares solution in the case of inconsistent data [30]. This property of the algorithm is especially beneficial for measurements that may be noisy or spoiled by artifacts due to the phase-retrieval step.

\subsubsection{Cone-beam reconstruction}

So far we have dealt with tomographic reconstructions for a parallel beam geometry. However, nearly all experiments performed in this work were carried out with divergent x-ray beams that enable a tunable magnification $M$. If the divergence angle is very small, e.g., for experiments performed at a synchrotron facility, where cone angles of focused beams are typically below $0.5^{\circ}$ or $9 \mathrm{mrad}$, the parallel beam approximation will hold quite well and reconstructions show hardly any artifacts due to cone-beam effects. For larger cone angles, the wrong approximation can lead to strong artifacts, as described in section 3.2.5.

Cone-beam artifacts can be avoided, if reconstruction algorithms are adapted to the different geometry. There are many 3D reconstruction algorithms that can account for complicated scanning geometries like spiral CT or arbitrary source- 
detector trajectories [29]. A widely used algorithm for the case of a planar detector and a point source, creating a divergent beam, was derived by Feldkamp, Davis and Kress (FDK), in which an approximate solution to the problem is given by a modified filtered back-projection [45]. In their algorithm, projections are weighted and filtered according to their relative location to the central slice of the illuminating cone and subsequently backprojected by cone-beam projection. It turns out that artifacts appear for structures that are far away from the cone center due to the approximate character of the algorithm [29]. Thus, for relatively small cone angles, the use of the FDK algorithm will improve the quality of the reconstruction. While due to symmetry reasons in the parallel beam case projections only need to be given for an angular range of $180^{\circ}$, for cone-beam tomography a larger angular range is needed to ensure that all voxels ${ }^{4}$ are seen from each side. It could be shown that a minimum range of $180^{\circ}$ plus the cone angle is sufficient, if the sinogram is filtered with suitable weights, known as Parker weights [135]. For this work, FDK reconstructions are performed using commercially available software by Andrei Bronnikov [27] and the freely available astra-toolbox [134].

Note that for ART, the algorithms presented in the previous section can be used for the reconstruction, as long as the projection and back projection operators defined by the system matrices $R^{i}$ are implemented such that they account for the cone-beam geometry.

\subsection{Artifacts in x-ray tomography}

As a tomographic image is the result of a reconstruction algorithm, imperfections during the recording process may lead to significant artifacts in the tomographic slices. This section will deal with some of the typical artifacts that can occur and with methods that can be used to avoid these artifacts or to remove them.

\subsubsection{Ring artifacts and ring-removal}

If in the tomographic projections some pixels are systematically spoiled, ring artifacts will occur that disturb the image impression. The wrong value of the pixel leads to a straight line in the sinogram, which is interpreted as a feature that always has the same distance to the center of rotation and is present under each angle, thus the reconstruction algorithm will produce a ring. Figure 3.5 shows an example with simulated data. For the simulation, a phantom consisting of spheres

\footnotetext{
4 A voxel is a made-up word for a volume cell, similar to a pixel being a picture cell.
} 

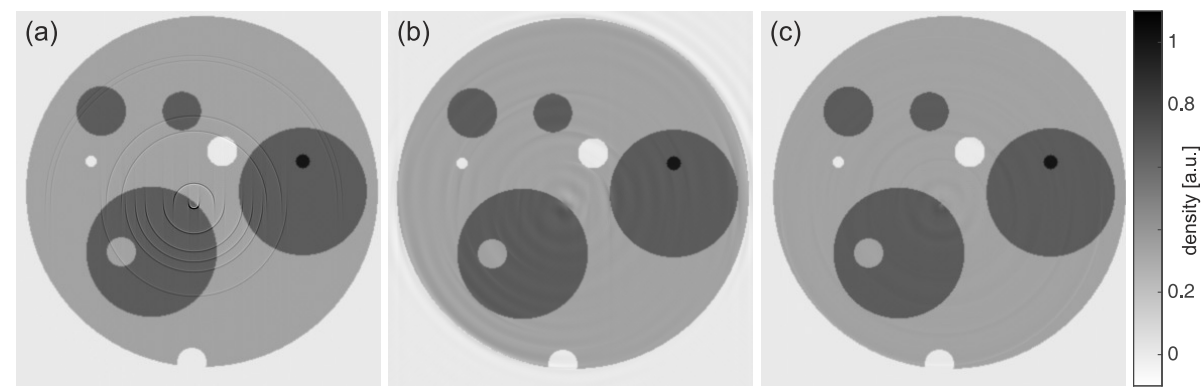

Figure 3.5: Ring artifacts in tomography. (a) shows a reconstructed slice without the application of any ring correction algorithm. (b) shows the same slice after applying a wavelet based filtering approach, where Daubechies 40 tap wavelets were used and the first three components are filtered with $\sigma=2.5$. (c) shows the same slice after the Fourier ring removal, where the sum over the angles is smoothed and the difference between smoothed and unsmoothed sinogram is subtracted.

with different sizes and density values between 0 and 1 is assumed. Projections are generated with the radon function implemented in Matlab and dead pixels are introduced by changing the values of single lines in the sinogram. In this case, a constant additive term, corresponding to two percent of the measured values is added for a total of 11 lines. The projections are simulated for an angular range of $\alpha \in[0,180]^{\circ}$ with $\Delta \alpha=0.25^{\circ}$ steps. Hence, if the spoiled data is reconstructed without any further correction, half rings will be introduced, as shown in Fig. $3.5(\mathrm{a})$.

Defect pixels that lead to ring artifacts may be caused by different physical effects. First of all, a pixel can be physically broken and thus will always provide wrong signals. In this case, best practice would be to mask the pixel and replace its value, e.g., by using an interpolated value of neighboring pixels. Even if no pixel is dead, there still may be ring artifacts in the reconstruction. In the image processing chain for phase-contrast tomography, an empty-beam correction and a dark-field correction is always performed (see section 4.4.2). If temporally unstable illuminations are used or calibration files are disturbed, e.g., by hot pixels due to high energy photons or detector noise, every projection will have spoiled values at this pixel since all projections are divided by the empty image. An easy approach to avoid such artifacts is to filter the empty image. For this thesis, the approach to remove outliers is as follows: A median-filtered image is used as a reference and the standard deviation $\sigma_{D}$ of the difference to the filtered image is calculated. 
If a certain pixel deviates more than a given threshold $t$, e.g., $t=2 \sigma_{D}$, it will be replaced by the median filtered value. To reduce the influence of detector noise, additionally multiple empty- and dark-images are recorded that can be used to obtain averaged correction files.

If despite all corrections ring artifacts are still present in a tomographic reconstruction, further algorithms acting on the sinogram or the reconstructed slice can be used to remove rings [29]. A widely used technique is based on wavelet filtering to remove straight lines in the sinogram [115]. To this end, the sinogram is decomposed in wavelet components and the first components in one image direction are filtered in Fourier space with a Gaussian function [115]

$$
g\left(\nu_{x}, \nu_{y}\right)=1-\mathrm{e}^{-\frac{\nu_{y}^{2}}{2 \sigma^{2}}}
$$

The value of $\sigma$ determines the width of the filter and has to be larger, if thicker lines have to be removed. This wavelet filtering approach can also be used to remove artificial lines in an image. The algorithm is applied to the simulated, spoiled data and the result is shown in Fig. 3.5(b). Another very simple and fast ring-removal algorithm works as follows: We summarize the sinogram over all angles to obtain a profile that depends on the coordinate perpendicular to the rotation axis. The profile is smoothed by removal of outliers. For this purpose, it is low-pass filtered in Fourier space and the difference between the smoothed and the unsmoothed version can be used to identify outliers, which are subsequently subtracted from the sinogram. This approach also is applied to the simulated data and the resulting slice is shown in Fig. 3.5(c). For the simulation used here, the latter approach performs better than the wavelet approach since rings are simulated as single pixel artifacts, producing strong spikes in the summed sinogram. If rings are broader, e.g., due to the used phase-retrieval algorithm, the wavelet based approach may deliver better results. However, it evidently would be beneficial to remove features leading to ring artifacts first and then perform the phase retrieval to avoid the enhancement of artifacts.

\subsubsection{Beam hardening}

If a polychromatic illumination with a large bandwidth $\Delta \lambda / \lambda$ is used to image a thick specimen, it may happen that low energies are fully absorbed, while the higher energies are still transmitted. This will lead to deviations from LambertBeer's law and will cause artifacts in the reconstruction, known as beam hardening. 
Especially strongly absorbing materials like metals or thick bones will show pronounced artifacts. If under some angles, x-rays are blocked by a strongly absorbing part, this leads to streak artifacts in the direction, where the absorption is highest. One way to overcome beam hardening is to reduce the bandwidth $\Delta \lambda / \lambda$, e.g., by applying absorption filters that transmit only higher energies. At third generation synchrotron facilities, where the radiation has a relatively small bandwidth due to the type of x-ray generation in undulators, beam-hardening artifacts are rarely observed. Additionally, weakly absorbing specimens are of interest for phasecontrast tomography and hence, beam-hardening will play a minor role in most cases. Note that artifacts similar to beam-hardening artifacts can also be observed with monochromatic radiation, if highly absorbing specimens are investigated and the measurement is limited by noise.

\subsubsection{Interior tomography}

If small features inside a large object are of interest, very large dimensions are necessary if the whole object has to be reconstructed. Let us consider for example the case of a whole mouse with a diameter of about $3 \mathrm{~cm}$. If sub-cellular structures in the sub-micrometer range are of interest and an isotropic voxel size of $30 \mathrm{~nm}$ is chosen, this means that a volume of $10^{6} \times 10^{6} \times 10^{6}=10^{18}$ voxels has to be reconstructed, which is not possible in the near future due to limitations of hardware and software. Instead, interior or local tomography can be performed, where not the whole object is imaged, but just a small region of interest (ROI) is present in the projections. It can be mathematically shown that for the $2 \mathrm{D}$ Radon transform, local projections are not sufficient to quantitatively reconstruct a local part of the object [116]. However, especially when the x-ray attenuation in the whole object is weak enough to obtain a suitable transmission signal, interior tomography can still be performed. In this section artifacts that can occur in interior tomography and approaches to correct them are introduced.

Figure 3.6 shows a simulation of a large phantom, consisting of $4096 \times 4096$ pixels, where the phantom used in Fig. 3.5 is placed into. For this object, 7200 projections with about 5800 pixels perpendicular to the rotation axis are simulated. Figure 3.6(a) shows the reconstruction of the full object. To simulate interior tomography, the projections are cropped symmetrically to obtain a sinogram that has only 600 pixels perpendicular to the rotation axis. If this data is used for tomographic reconstruction, the slices will be overlaid by spurious low spatialfrequency variations, as shown in Fig. 3.6(b). While many sophisticated methods 
(a)

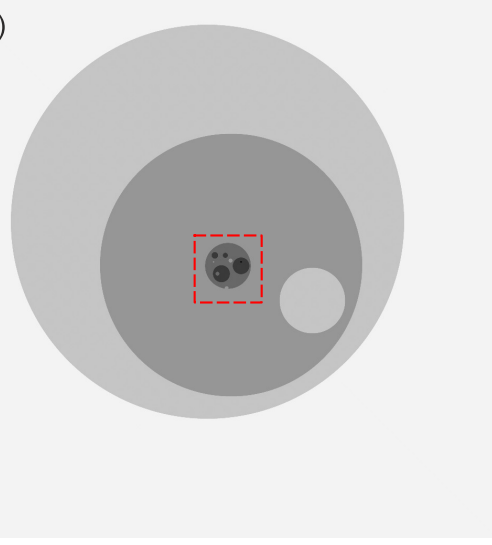

(c)

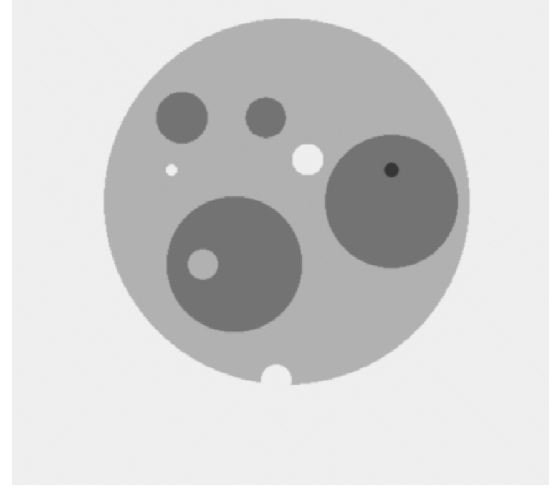

\section{(b)}

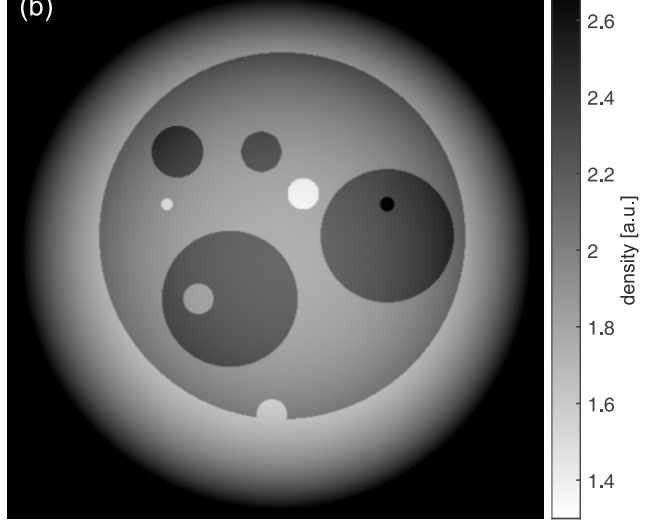

(d)

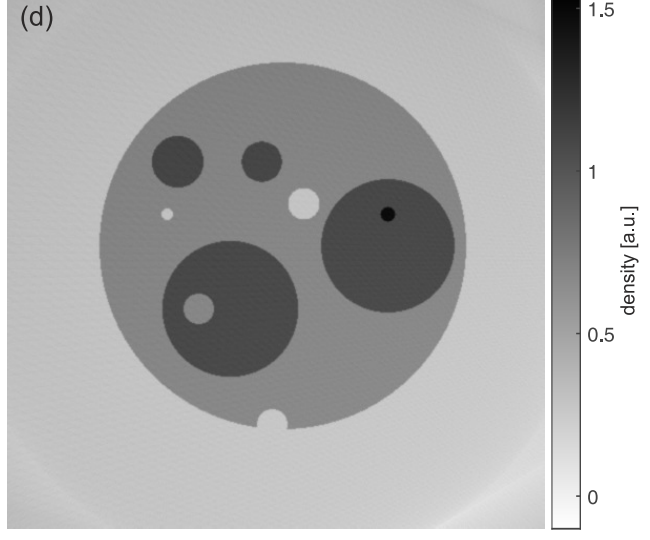

Figure 3.6: Artifacts occurring in interior tomography: (a) shows the full phantom used for the simulation, where the dashed line marks the region of interest (ROI) that is shown as a magnification in (c). (b) shows the reconstruction of a ROI dataset that contained only 600 of the 5800 pixels perpendicular to the rotation axis without further corrections, resulting in a strong distortion of low spatial frequencies. (d) shows the reconstruction of the same ROI, but in each projection boundary values were replicated to enlarge the measurement. Additionally, only 360 instead of 7200 projections were used, which leads to a slight high-frequency non-uniformity in regions of constant density. The padding approach reduces the low-frequency artifacts, but may also change the quantitative values of the reconstruction. The color axis for (a) ranges from -0.9 (white) to 1.3 (black). 
for interior tomography exist $[60,189,191]$, a very simple approach is to smoothly extend projections [94]. The projections used for Fig. 3.6(b) are padded to have a length of about 1200 pixels by replicating their boundary values. If the padded projections are used for the tomographic reconstruction, the low-frequency artifacts can be suppressed (see Fig. 3.6(d)). However, compared to the zoom into the original reconstruction shown in Fig. 3.6(c), slight deviations in the absolute values can be observed. In the case shown here, these deviations can be reduced even more by further enlarging the projections. As the padding factor influences the reconstructed values, quantitative information about the absolute values cannot be expected from interior tomography. However, relative density changes still approximate the true object quite well [60].

\subsubsection{Sampling artifacts}

Note that for the reconstruction shown in Fig. 3.6(d), only 360 angles are used to obtain the central $512 \times 512$ pixels, which has no significant influence on the lowfrequency structure of the image. As structures outside the FOV are also contained in the projections, additional high-frequency artifacts may be introduced, if only a few projections are used. For a traditional tomographic scan, Nyquist sampling of the reconstruction is achieved, if the number of projection $N_{\alpha}$ is determined by $[29]$

$$
N_{\alpha} \geq N_{y} \pi / 2
$$

where $N_{y}$ is the number of pixels perpendicular to the rotation axis that is necessary to display the object. However, for interior tomography it has been shown that the number of projections $N_{\alpha}$ has to be chosen based on the dimensions of the whole object to ensure proper sampling [94]. Hence, for the reconstruction in Fig. 3.6(d) the (already very small) artifacts would further decrease if about 6000 projections are used. One way to still allow the use of fewer projections is to record the data in a continuous mode, where the rotational motion is not stopped to capture an image. In this fly-scanning mode, structures that are farther from the rotation center are smeared out, which will reduce artifacts due to limited angles. However, undersampling artifacts will not be limiting the reconstruction quality in most cases for experiments in this thesis. 


\subsubsection{Geometry artifacts}

In most cases it is assumed that the rotation axis, which we defined to be the $z$-axis, is located in the vertical center-line of the image. If experimental data is recorded and the physical rotation axis does not lie exactly in the center line, this leads to artifacts in the reconstruction as shown in Fig. 3.7. Depending on the angular range, the appearance of the artifacts may vary. For a range of $360^{\circ}$, edges will be blurred and dots will transform into concentric circles. In the simulation in Fig. 3.7 an angular range of $180^{\circ}$ is used and detector offsets of -1 , +5 and -10 pixels are assumed for Fig. 3.7(a), (b) and (c), respectively. Due to the wrong position of the rotation axis, the spheres are not closed anymore and half-moon like structures appear, which are evidently stronger for larger shifts. Experimental data can be corrected by moving all values of the projections, e.g. using the circshift function implemented in Matlab. To minimize artifacts, several offsets can be tried out. For the best value, circular structures should show no streak artifacts and the contrast, i.e., the average difference between maximum and minimum value is maximized. If the rotation axis has a different roll angle than the detector (see Fig. 3.9(b)) the position of the physical rotation axis will be different for every vertical position along the $z$-axis. Hence, finding the rotation axis for several $z$-positions can help to calculate a residual roll angle, which can be used to correct the projection images by applying numerical rotation algorithms.

For a parallel beam reconstruction, only the position of the rotation axis has to be shifted to match with the vertical center line. By contrast, for the cone-
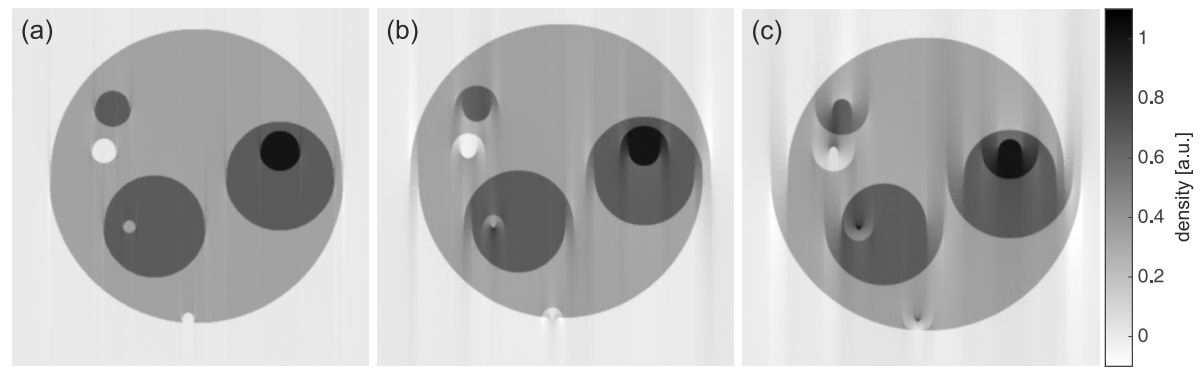

Figure 3.7: Artifacts due to a wrong position of the rotation axis: Simulated projections over a range of $180^{\circ}$ were shifted such that the center of the rotation axis is not in the center of the image anymore. (a) shows a reconstruction where all projections are shifted by -1 pixel. In (b) the projections are shifted by +5 pixels and for (c) all projections are shifted by -10 pixels. 

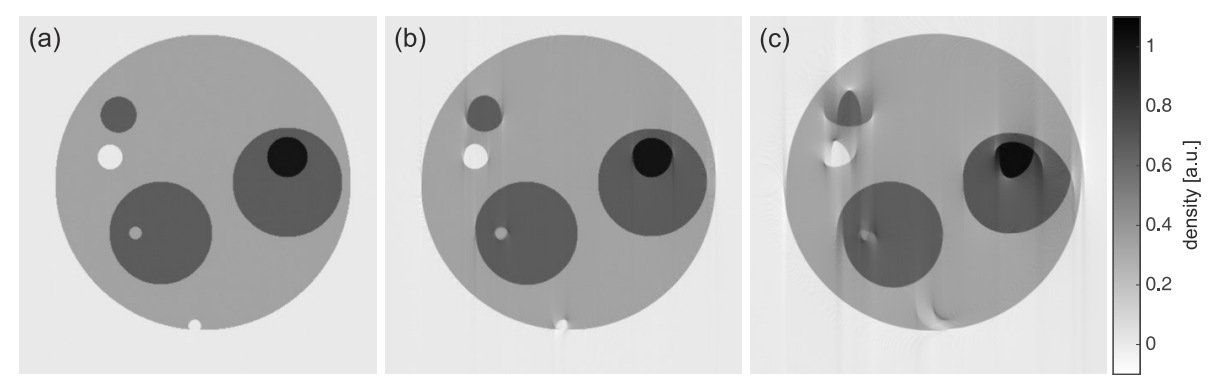

Figure 3.8: Artifacts due to treating cone-beam as parallel beam geometry: The geometry was simulated as described in the main text and projections are simulated for different fields of view that can be described by the cone angle. (a) shows a parallel beam reconstruction of cone-beam projections obtained with a cone angle of about $0.49^{\circ}$, where no artifacts are visible. (b) shows the reconstruction with $4.9^{\circ}$ cone angle, where artifacts start to appear. In (c) this angle is $24.1^{\circ}$ leading to heavy distortions. Note that the artifacts are similar to those shown in Fig. 3.7, however, the direction of the open half circles is different in the same slice.

beam case more details about the geometry are necessary to achieve artifact-free reconstructions. Necessary parameters are the source to object distance $x_{01}$, the object to detector distance $x_{12}$ and the detector pixel size $p=p_{x}=p_{y}$. By using the two distances $x_{1}$ and $x_{2}$, an effective voxel size $\Delta v=p / M$ can be calculated, which is the detector pixel size $p$ divided by the geometric magnification $M=\left(x_{01}+x_{12}\right) / x_{01}$. Additionally, the detector has to be centered in both the horizontal and the vertical direction. However, the centering in vertical direction has not such a high influence on the reconstruction quality as the centering in horizontal direction, where the rotation axis is moved.

Figure 3.8 shows a simulation of a different type of typical cone-beam artifacts, for which projections obtained in cone-beam geometry are reconstructed under the assumption of a parallel beam. For the simulation that was implemented using the astra toolbox [134], $x_{01}=x_{12}=15 \mathrm{~cm}$ is chosen so the magnification is $M=2$. Different pixel sizes $p$ are assumed for the same object, leading to different fields of view and thus to different cone angles. For Fig. 3.8(a), the pixel size is $p=5 \mu \mathrm{m}$ resulting in a cone angle of about $0.49^{\circ}$, where the parallelbeam reconstruction shows no visible artifacts. A larger cone angle of about $4.9^{\circ}$ is obtained with $p=50 \mu \mathrm{m}$. In the corresponding reconstruction in Fig. 3.8(b), vertical streak artifacts can be observed, similar to a shifted rotation axis (cf. Fig. 3.7). However, these artifacts cannot be compensated by shifting the values, as 
their direction and strength differs depending on their position in the object. In Fig. 3.8(c) the pixel size is $p=250 \mu \mathrm{m}$, leading to an angle of about $24.1^{\circ}$ and thus heavy artifacts are introduced in the object. To avoid cone-beam artifacts, it is crucial to know the distances $x_{01}$ and $x_{02}$ and to use cone-beam reconstruction as the FDK algorithm presented before.

\subsubsection{Motion artifacts}

The worst case for tomographic imaging is a change within the sample during the measurement. For strong alterations of the sample, e.g., the creation of air bubbles in a liquid environment, it will be almost impossible to reconstruct the underlying object without artifacts. If only little movement of some features occurs during the measurement, artifacts similar to a shifted rotation axis (cf. Fig. 3.7) can be present in the reconstruction. Motion can also lead to doubling or blurring of structures [23]. As the total dose necessary for a noise-free image can be distributed over the whole tomographic scan, known as dose-fractionation in the literature [108], the easiest way to avoid motion artifacts, is to speed up the measurement. This can be achieved, e.g., by choosing smaller exposure times, such that the single projections show more noise, which will be averaged out in the reconstruction. However, a practical limit is rapidly reached as the mechanics are not arbitrarily fast and a certain dose is necessary for alignment and to recognize fine structures. A better treatment of motion and its correction in tomographic measurements is currently a hot topic in tomographic research. Recently an approach to detect and correct a global affine motion was presented in [121].

\subsection{Alignment procedures and algorithms}

In order to avoid any kind of artifacts in a tomographic reconstruction, the alignment of the rotation axis with respect to the detector is a crucial step. In particular, the rotation axis needs to be centered in the FOV of the detector, and pitch and roll angle $(\theta$ and $\phi)$ between detector and rotation axis have to be corrected. While the latter can be corrected numerically by rotating all the images, the pitch angle $\theta$ needs to be corrected before the measurement is carried out. If the detector is placed far away from the source and only small angles $\theta$ have to be corrected, a vertical movement of the detector can be equivalent to a tilt of the rotation stage. A horizontal movement of the detector would analogously change the zero position of the tomographic rotation. 

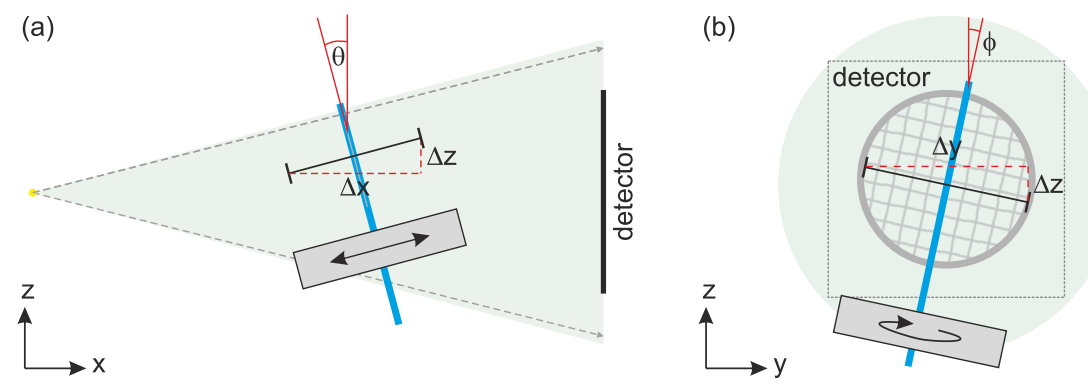

Figure 3.9: Procedures to align the pitch and roll angle of the rotation axis: (a) To align the pitch angle $\theta$, a translation directly above the rotation axis is used to move the sample in $x$-direction over a distance $\Delta x$. For a non-vanishing pitch angle $\theta \neq 0$ the measured image will show a vertical movement $\Delta z$ that can be used to calculate the angle $\theta$. (b) The alignment of the roll angle $\phi$ can be done by a rotation around the tomographic axis. If the roll angle is not the same as for the detector, features with a distance $\Delta y / 2$ to the rotation axis will show a vertical movement $\Delta z$ that can be used to calculate the angle and to subsequently either rotate the detector, the sample stage or the measured images.

In the following we will list the typical alignment steps for a tomographic measurement in a divergent beam. To do so, a flat test structure is needed, to avoid different magnifications of the same object. For this thesis, a structure consisting of different grids and brass swarf glued to a polypropylene foil is used for the alignment. The geometric situation is sketched in Fig. 3.9. We assume that the rotation axis can be moved in $y$-direction and that above the rotation axis two perpendicular motors are located to enable a motion perpendicular to the tomographic rotation axis.

For the alignment, the following steps have to be carried out:

- Finding and centering the rotation axis:

- Two images are recorded at $0^{\circ}$ and at $180^{\circ}$ by rotating the tomographic axis.

- The same feature has to be identified in both projections, yielding coordinates $\left(y_{1}, z_{1}\right)$ at $\alpha=0^{\circ}$ and $\left(y_{2}, z_{2}\right)$ at $\alpha=180^{\circ}$.

- The physical rotation axis is located at $\left(y_{1}+y_{2}\right) / 2$ and can be changed with a positioner below the rotation axis such that it coincides with the vertical center-line of the image.

- The procedure can be repeated for several features for accuracy. 
- Centering the test structure in the rotation axis:

- Move the test structure into the center of rotation, i.e., the center of the image, for a rotational angle of $\alpha=0^{\circ}$.

- Do the same for $\alpha=90^{\circ}$.

- If two motors perpendicular to each other are present, any other angle can be used to center the sample, too. However, angles close to $\alpha=90^{\circ}$ will be most accurate.

- Aligning the pitch angle $\theta$ in a divergent beam:

- Move the object along the optical axis over a distance $\Delta x=x_{2}-x_{1}$ using a motor above the rotation stage

- Record two images at $x_{2}$ and $x_{1}$.

- If features in the images change their height by $\Delta z$ (besides magnification effects), the pitch angle $\theta$ is given by (see Fig. 3.9(a))

$$
\theta=\arctan \left(\frac{\Delta z}{\Delta x}\right)
$$

- The two images will have a different magnification, so observation of height changes may be difficult. However, for one point on the optical axis, no change in magnification can be observed in the measured images, which can be seen best with regular grids as a test structure.

- Finally the stage is tilted, so that the beam-center point is centered in the detector, which can be checked by repeating the measurement.

- Aligning the roll angle $\phi$ :

- Again two images at $0^{\circ}$ and $180^{\circ}$ are recorded.

- The coordinates of a feature of interest are used to calculate $\Delta z=z_{2}-z_{1}$ and $\Delta y=y_{2}-y_{1}$

- The roll angle can thus be calculated via (see Fig. 3.9(b))

$$
\phi=\arctan \left(\frac{\Delta z}{\Delta y}\right) .
$$

- The procedure is repeated for several points for accuracy.

The alignment can also be automatized to some extent. Especially the first part to find and center the rotation axis can be performed automatically. To this end the image at $180^{\circ}$ is flipped along the $z$-axis and registered to the image at $0^{\circ}$ by 
using an FFT-based correlation algorithm presented in [62]. If the effective pixel size is known, the shift can be used to calculate the distance, by which the rotation axis needs to be moved. For the alignment of the pitch angle, an algorithm was written that measures both images at $x_{1}$ and $x_{2}$. Subsequently, the quotient of both images is displayed, where the user has to click at the point of the "beam center", where the contrast vanishes. The coordinates of this point, together with the knowledge about the geometry can then be used to correct either the pitch angle or the vertical position of the detector automatically. 



\section{Experimental realization}

The generic setup for phase-contrast tomography measurements, as sketched in Fig. 1.1, consists of three main elements: An x-ray source that produces (partially) coherent x-ray radiation, a motorized sample stage that has to position a specimen in the beam and that enables rotational movement for tomographic measurements and last the detector, which after some free-space propagation measures the intensity distribution of the x-rays that can be used for phase retrieval and subsequent tomographic reconstruction. This chapter deals with the practical aspects and gives an overview about the different experimental setups that were used and improved during this work.

\subsection{X-ray generation}

Since the first discovery of x-rays in the 19th century by Röntgen, great advances in the generation of x-rays could be realized. Two basic principles are mainly responsible for x-ray formation: The interactions of free electrons with those bound to an atom and the accelerated motion of a charge density [3]. If a charged particle with high energy approaches a solid metal, it will be able to excite or ionize the atoms. Electrons of another energetic shell can refill the gap and during the transition, they will emit the energy difference as electromagnetic radiation. If this happens to core electrons the energy difference will be in the $\mathrm{keV}$ range, hence $\mathrm{x}$-rays are generated. This kind of radiation is known as characteristic radiation. Charged particles that hit a metal anode will also experience electric forces that will change their propagation direction and thus they are accelerated. As an accelerated charge also yields electromagnetic radiation, so-called bremsstrahlung will be created. The energetic spectrum of the bremsstrahlung goes up to the maximum kinetic energy of the electrons. These two principles are used in $\mathrm{x}$ ray sources, where in an evacuated tube, electrons, emitted by a cathode, are accelerated to multi-keV regions and upon impact on an anode, characteristic $\mathrm{x}$ rays and bremsstrahlung are produced. An important measure of the quality of 
an $\mathrm{x}$-ray source is the brilliance [3]

$$
\text { Brilliance }=\frac{\text { photons } / \text { second }}{(\mathrm{mrad})^{2}\left(\mathrm{~mm}^{2} \text { source area }\right)(0.1 \% \text { bandwidth })} .
$$

A higher brilliance allows using more coherent illumination for an experiment. Due to the large polychromaticity in laboratory sources, one usually states the brightness, which is the brilliance without the normalization by the bandwidth.

In order to obtain partially coherent x-rays, a small source size is necessary that can be achieved by focusing the electrons with magnetic lenses. However, more than $99 \%$ of the total energy will only heat the anode, which in the end will lead to melting or evaporation of the anode [129]. Recently, a novel kind of x-ray source was introduced by a spin-of of the KTH Royal Institute of Technology in Sweden named Excillum, in which liquid metal is used to create x-rays [128]. To this end, a special metal alloy Galinstan, consisting of gallium, indium and tin, which is liquid at room temperature, is injected with high pressure into vacuum. Thus, melting the anode is no problem anymore and even if the metal will be evaporated it has no big influence as long as the metal debris does not hit the cathode. With currently available liquid-metal jet sources, the brightness is up to 10 times higher compared to classical x-ray tubes. A new prototype source will offer an increased brightness, which is more than 50 times higher than in classical tubes in the near future [98].

A completely different way to create x-ray radiation is the use of large-facility accelerators [3]. Here, electrons are accelerated to energies in the range of some $\mathrm{GeV}$ and hence, they will move with nearly the speed of light. If they traverse magnetic fields, they will encounter a force that leads to an accelerated motion. Thus, electromagnetic radiation is emitted. By relativistic effects, the energy range of the radiation will correspond to that of x-rays. A bending magnet, usually used to keep the electrons on a closed circle, emits radiation in a broad energy range. Contrary, so-called insertion devices like wigglers or undulators, where periodic, alternating magnetic fields are used to deflect the electrons, produce much more brilliant radiation with a lower bandwidth [3]. For experiments relevant for this thesis, $\mathrm{x}$-rays are always generated in undulators. 


\subsection{X-ray detectors}

A very important part of a phase-contrast experiment is the x-ray detector. Several kinds exist today that all have different advantages and drawbacks. In this section, we will give a short overview of available devices. A major criterion that influences the choice of a detector is the point-spread function (PSF). This function tells how a single point will be spread and thus it is a measure for the achievable resolution. In addition to the PSF, further functions describing the resolution are introduced: The line-spread function (LSF) and the edge-spread function (ESF). The LSF can be obtained, if an infinitesimally thin line is convoluted with the PSF. The ESF is the anti-derivative of the LSF, which usually is described by an error function

$$
f(x)=c \cdot \operatorname{erfc}\left(\frac{\mu-x}{\sqrt{2} \cdot \sigma}\right)+d .
$$

Here, $c$ is some arbitrary scaling parameter, $d$ some offset, $\mu$ is the position of the edge and $\sigma$ the standard deviation of the underlying Gaussian LSF. A quantity that is typically stated to quantify the resolution is the full width at half maximum (FWHM) of the PSF or LSF, which can be obtained by

$$
\mathrm{FWHM}=2 \cdot \sqrt{2 \cdot \ln (2)} \cdot \sigma
$$

\subsubsection{Direct detectors}

In a direct detector, the x-ray radiation interacts with some material (e.g. silicon, gallium arsenide or cadmium telluride) mainly due to the photoelectric effect. This leads to the creation of free electrons, which are measured by the electronics. A widely used detector type is a charge coupled device (CCD). In a CCD the charge is created in a pixel and sequentially transferred over other pixels by the electronics to an amplifier and readout module. As hard x-rays have energies in the range of some $\mathrm{keV}$, many electrons are created upon impact of an x-ray photon. A CCD has a limited full-well capacity so the dynamic range of an x-ray CCD is very limited. Typically less than 100 hard x-ray photons per pixel can be measured until a pixel is saturated. Another disadvantage is that, if many pixels are needed, e.g., a matrix consisting of 1300 by 1300 pixels, a typical readout time is in the range of 1 to 2 seconds [1]. Hence, averaging to increase the number of photons per pixel is a time consuming process and this type of detector is thus mostly used for soft x-rays, where a single photon creates less electrons.

A better detection principle for x-ray radiation is realized in photon-counting 
detectors $[26,100,141]$. Here, every pixel has additional electronics that is able to discriminate and count the charge pulses. This enables a noise-free readout and the dynamic range is only limited by the speed of the electronics. Additionally, the discriminator allows measuring only photons above a certain energy. For example the Pilatus detector (Dectris, Baden, Switzerland) allows a to count $10^{6}$ photons $/ \mathrm{s}$ while keeping the sensitivity for single photons. However, this comes at the price of relatively large pixels with a size of $p=172 \mu \mathrm{m}$ and inter-module gaps. Another photon counting detector was developed by the medipix consortium [100]. For the currently available systems called timepix the pixels are built in a similar way, but the design is more compact and a pixel size of $p=55 \mu \mathrm{m}$ is achieved. Due to the detection principle, photon counting detectors have a very good PSF of about 1 pixel. For very high flux densities, they may not be fast enough. If two photons hit the detector with a very short time delay, they may be interpreted as a single photon. Integrating detectors offer a good compromise between a photon counting detector and a CCD, where measurements at high flux are possible and single photon sensitivity can be maintained [72].

If a single charge pulse is analyzed, the number of electrons generated is proportional to the energy of the radiation. Together with a multi-channel analyzer, this principle can be used to build energy sensitive detectors. In contrast to other direct detectors, these detectors in most cases are point detectors and no spatial resolution is achieved. However, if calibrated against known reference energies, they enable the measurement of x-ray spectra and thus the photon energy spectrum can be analyzed or elemental information from a sample can be obtained. With a new detector type called Maia, 96 parallelized spectroscopic measurements are possible at once, enabling real time spectroscopic imaging [152].

\subsubsection{Indirect detectors}

The most common detection principle for hard x-rays is based on converting the $\mathrm{x}$ rays to visible light using a scintillating material. If x-ray photons hit a scintillator, electrons are created and by secondary effects these can stimulate the emission of visible light. The created light can be captured by a classical CCD or CMOS camera. While in a CCD camera, the generated charge is transferred pixel-bypixel and thus the readout is very slow, in a CMOS camera every pixel has its own amplifier and signals can be transferred much faster. The resulting reduced active area can be compensated, e.g., by using micro lenses. Recently a so-called scientific CMOS (sCMOS) chip has been presented that combines low noise readout, a small 
(a)

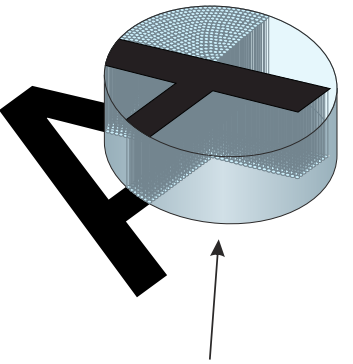

(b)

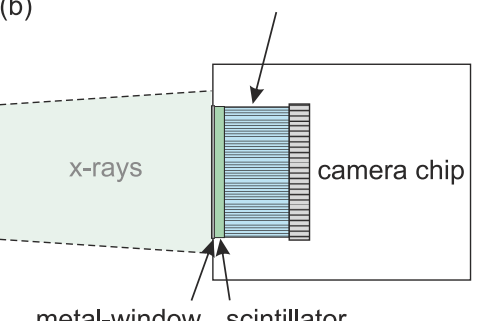

(c)

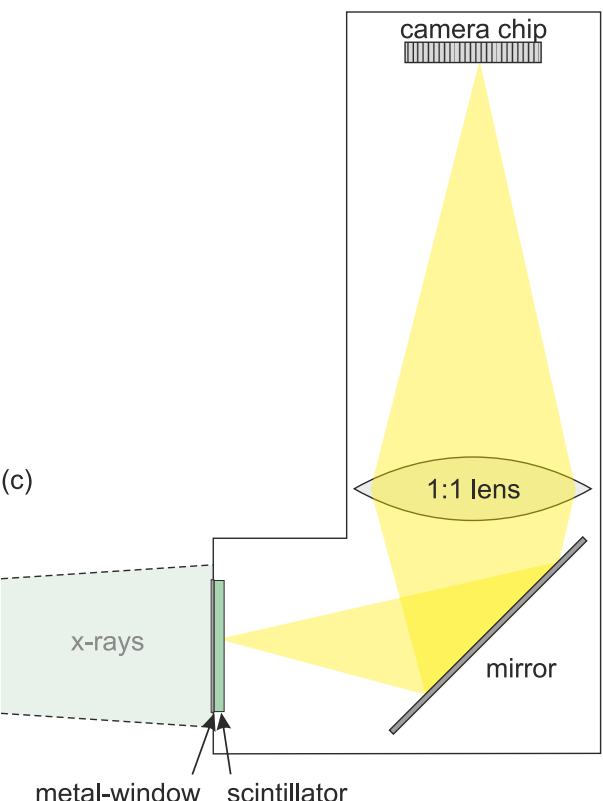

Figure 4.1: Two principles of indirect detectors. (a) shows the basic principle of a fiber optic plate (FOP) that transmits light from one plane to another with a high numerical aperture. Image based on [66]. (b) shows the detection principle using a FOP bonded to a scintillator and the camera chip, to enable an efficient 1:1 mapping. (c) shows the principle of a lens coupled detector, where the light, created in a scintillator and reflected on a mirror, is focused to a camera chip by a 1:1 lens. In both cases a thin metal window (aluminum or beryllium) is used to transmit $\mathrm{x}$-rays and block the visible light.

pixel size of $p=6.5 \mu \mathrm{m}$, relatively high quantum efficiency of up to $70 \%$ and fast frame rates up to $100 \mathrm{~Hz}$ and a high resolution of $2048 \times 2048$ or more pixels $[48,67]$. The main challenge in an indirect detector is the light transfer from the scintillator to the camera chip.

A rather easy and flexible approach is to use a lens-coupled detector. To this end, the generated light is reflected at a mirror and a lens focuses the light to the camera as shown in Fig. 4.1(c). This has the advantage that the lens and the camera electronics can be shielded to protect them from the x-ray radiation. Alternatively, there are variants that use microscope objectives with high numerical aperture, which have to be placed close to the scintillator. However, without the protection by the mirror, the lens system gets brown after some exposure to the $\mathrm{x}$-rays, which reduces the transmission drastically. Hence, for this detection prin- 

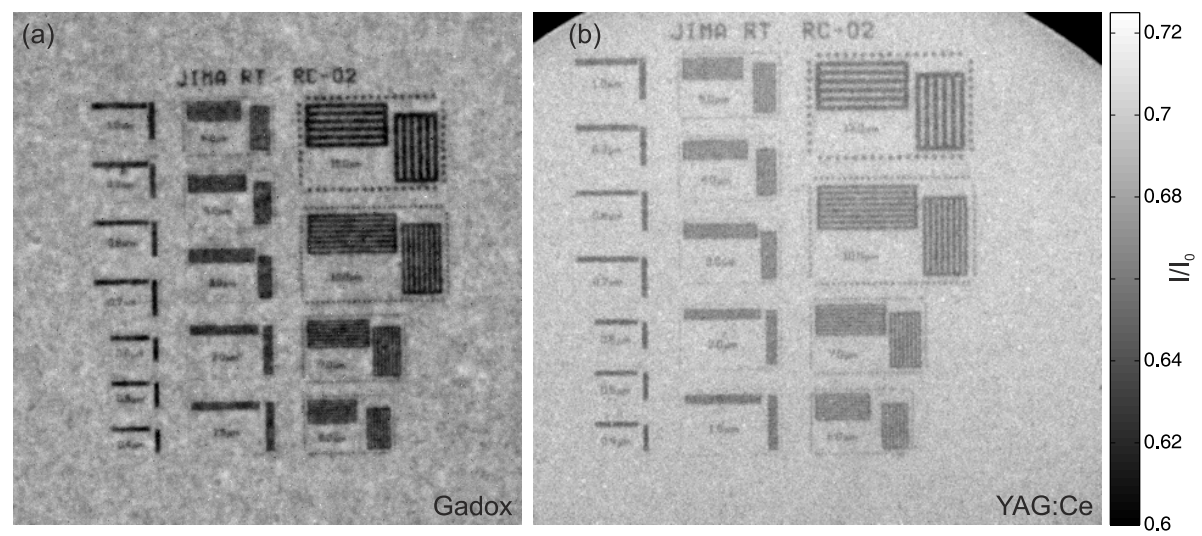

Figure 4.2: Comparison between (a) an amorphous gadox scintillator and (b) a single crystal YAG:Ce scintillator, both coupled with a FOP to an sCMOS camera and illuminated with the same energy spectrum. Both images are dark field and empty-beam corrected and outliers due to high energy photons are removed. The image in (b) was measured at a higher geometric magnification and rescaled to have a similar pixel size as the image in (a), as the YAG scintillator was too thick to achieve a high resolution.

ciple, the rather expensive microscope objectives are used as consumables. The resolution only depends on the optical system behind the scintillator and, by using single-crystal scintillators, very good PSFs below $1 \mu \mathrm{m}$ are possible [42]. The lens-coupling approach allows the flexible changing of scintillators or lenses. However, much of the generated light is lost as the numerical aperture of the imaging system is rather low, at least for low magnifications. A higher numerical aperture can be achieved for larger magnifications, but since the FOV is also reduced, this approach is not very efficient if the illuminating beam is larger than the area seen by the detector.

A more efficient approach is the use of a fiber-coupled detector, as sketched in Fig. 4.1(b). Here the light is transferred via a fiber-optic plate (FOP), whose working principle is depicted in Fig. 4.1 (a). A FOP consists of many small waveguiding channels, i.e., channels with a core material surrounded by a cladding, where the light is guided in the core by total reflection at the core-cladding transition. If a camera chip is equipped with a FOP, a scintillator can be deposited on it. This principle is widely used to produce so-called flat-panel detectors relevant for medical imaging [161]. However, these detectors typically have large pixels of $p=50 \mu \mathrm{m}$ or more. Very recently, this approach has been used to equip 


\begin{tabular}{llllll}
\hline name & detection principle & manufacturer & pixel matrix & pixel size & fps \\
\hline Pilatus & photon counting & Dectris & $487 \times 619$ & $172 \mu \mathrm{m}$ & 500 \\
DFP & CMOS flat panel & Dexela & $1944 \times 1536$ & $75 \mu \mathrm{m}$ & 26 \\
Timepix & photon counting & XIE & $768 \times 512$ & $55 \mu \mathrm{m}$ & 5 \\
LCX & direct illum. CCD & Princeton & $1340 \times 1300$ & $20 \mu \mathrm{m}$ & 0.55 \\
Pirra & sCMOS, gadox & Photonic Science & $2048 \times 2048$ & $6.5 \mu \mathrm{m}$ & 18 \\
Zyla & sCMOS, blank FOP & Andor & $2560 \times 2160$ & $6.5 \mu \mathrm{m}$ & 100 \\
Pandora & sCMOS, gadox & Photonic Science & $1920 \times 1080$ & $6.5 \mu \mathrm{m}$ & 18 \\
Frelon & lens coupled CCD & Optique Peter & $2048 \times 2048$ & $14^{*} \mu \mathrm{m}$ & $\sim 10$ \\
PCO & lens coupled CCD & Optique Peter & $2048 \times 2048$ & $7.4^{*} \mu \mathrm{m}$ & $14^{* *}$ \\
Xsight & lens coupled CCD & Rigaku & $3326 \times 2504$ & $0.54 \mu \mathrm{m}$ & 0.3 \\
MCA & spectroscopic & Phywe & 1 & n.a. & n.a. \\
\hline
\end{tabular}

Table 4.1: Comparison of different detectors - * The camera has the stated pixel size, but depending on the optical magnification (from 1.25-fold to 20-fold), a smaller effective pixel size in the range of $(0.37 \ldots 11.2) \mu \mathrm{m}$ is achievable. ** The pco.1400 camera is able to capture 14 frames/second (fps) into an internal memory. For continuous operation about 1 frame/second can be achieved.

sCMOS cameras with a scintillator [174]. To achieve high resolutions and get rid of afterglow, scintillators consisting of gadolinium oxysulfate $\left(\mathrm{Gd}_{2} \mathrm{O}_{2} \mathrm{~S}\right.$, gadox $)$ are used that are available as grains of different size, e.g., between 1 and 2 micrometers. Such a scintillator can be directly deposited on a FOP by volatilizing an emulsion of the grains. However, with the high resolution that the sCMOS camera is capable off, the grainy structure of the scintillator will lead to a non-uniform response of the detector, even after flat-field correction (see Fig. 4.2(a)). To circumvent these limitations, single crystal scintillators can be used instead. These show a much better uniformity after empty-beam correction as can be seen in Fig. 4.2(b). For this comparison, a $50 \mu \mathrm{m}$ thick single-crystal yttrium-aluminum-garnet $\left(\mathrm{Y}_{3} \mathrm{Al}_{5} \mathrm{O}_{12}\right.$, YAG) scintillator doped with cerium (Ce) was coupled with immersion oil to an sCMOS camera (Zyla HF5.5, Andor Technologies, United Kingdom) [6]. As a reference, a closed x-ray detector (Photonic Science, Robertsbridge, United Kingdom), where a $15 \mu \mathrm{m}$ thick gadox screen is coupled with a FOP to a similar sCMOS chip is used. Both measurements are performed at the JuLiA instrument described before under same x-ray source conditions. A test pattern (Jima TR RC-02, Japan Inspection Instruments Manufacturers Association, Japan) is positioned at $x_{01}=167 \mathrm{~mm}$ behind the source. Raw, empty and dark images are averaged results of $10 \times 1 \mathrm{~s}$ exposure and the detector was placed at $x_{02}=231 \mathrm{~mm}$ for (a) and at $x_{02}=392.8 \mathrm{~mm}$ in (b). The resulting difference in pixel size is compensated by downsampling the image in (b). 
The image obtained with the gadox scintillator (Fig. 4.2(a)) shows grainy structures in empty regions, which are caused by the scintillator type. In contrast, the single-crystal scintillator delivers a much more uniform image. Additionally, the transmission in (b) is slightly higher, which can be explained by a better absorption of the scintillator at higher photon energies. Several scintillators were tested during this work and in collaboration with Hamamatsu Photonics, an optimized x-ray sCMOS camera, equipped with a $20 \mu \mathrm{m}$ thin lutetium aluminum garnet (LuAG) scintillator that is doped with cerium will be produced in the near future. In Tab. 4.1 some parameters of different $\mathrm{x}$-ray detectors are compared. The most relevant detectors for this work are the fiber-optically coupled sCMOS cameras "Pirra" and "Pandora", the lens-coupled "Frelon" camera and the photon counting "Timepix" detector.

\subsection{Laboratory setup JuLiA}

A laboratory instrument called "Just a liquid anode" (JuLiA) for phase-contrast tomography is built using a state-of-the-art liquid-metal jet source (JXS D2, Excillum, Stockholm, Sweden). After a propagation-based phase-contrast tomography setup was built with the first commercial prototype of a liquid-metal jet source, described in detail in [13], a successor with a new version of the metal-jet source was built during this work. Figure 4.3 shows a photograph of the setup, with the $\mathrm{x}$-ray source, the motorized sample tower and an sCMOS detector with gadox scintillator. The setup is built on two parallel aluminum profiles to allow a high flexibility in positioning of all components. For very long propagation distances, an evacuated flight tube can be inserted, to reduce absorption of the x-rays in air. A fully motorized sample tower enables the precise positioning of a rotation axis in all three dimensions. Above the rotation, two small positioners are available, to allow centering of the object or a region of interest (ROI). The setup is built such that the tomographic axis corresponds to the $z$-axis. To enable a finer alignment of the pitch angle (see Fig. 3.9), the detector can be moved in $y$ - and $z$-direction. All movements of the motors are controlled with a diffractometer control-software called spec (Certified Software, Cambridge, USA) that allows the easy implementation of motor controllers and point detectors. Furthermore with an integrated macro language, it is easy to extend the capabilities and all available detectors are controlled via specific macros. Hence, complete experiments can be run as a batch script from spec.

While the basic principle of the x-ray source is still the same as in [13], many fea- 
tures like automatic spot-size calibration or spot position have been implemented in the new version of the source. Electrons can be accelerated with a voltage up to $U=160 \mathrm{kV}$ and are focused by magnetic lenses to a spot with a size down to $4 \mu \mathrm{m}$ on the liquid-metal jet, which has a diameter of about $180 \mu \mathrm{m}$. Depending on the position of the electron beam on the metal jet, the effective x-ray source size can be different, as illustrated in Fig. 4.4. In fact, the electron spot size in $x$-direction only has a significant influence, if the electron beam is positioned off the center, as in Fig. 4.4(b). To allow increased electron-beam powers for x-ray

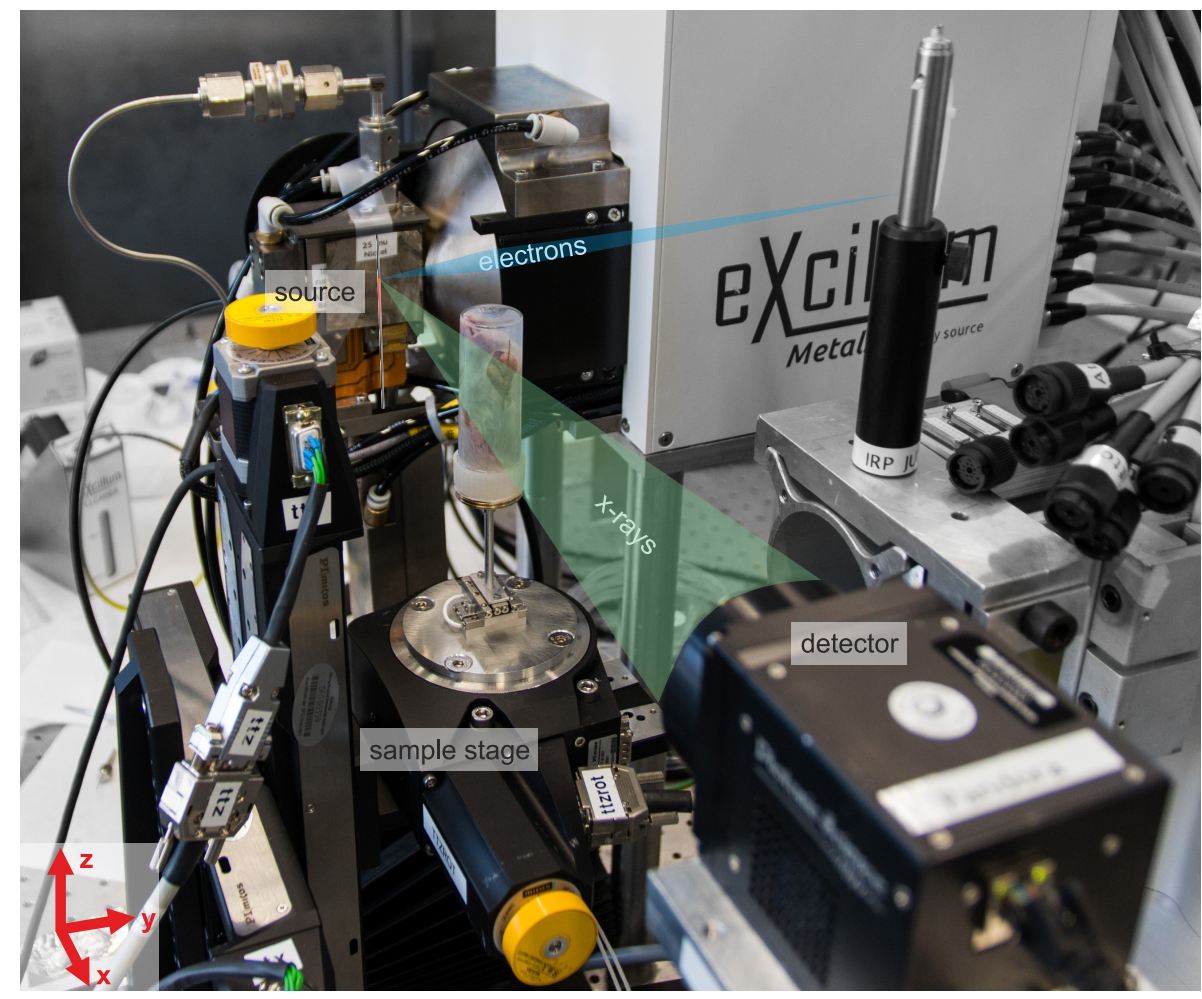

Figure 4.3: Photograph of the experimental setup at JuLiA: Electrons (blue) are accelerated with $70 \mathrm{kV}$ and focused to a spot down to $4 \mu \mathrm{m}$ on a liquid-metal jet where $\mathrm{x}$-rays (green) are generated. The x-rays can be filtered by absorption foils and will pass the sample tower that is able to move the rotation axis in $\mathrm{x}$-, $y$ - and z-direction with motorized linear stages. Above the rotation, two small positioners to center the sample in $x$ - and $y$-direction are available. A detector can be positioned with linear tables in $y$ - and $z$-direction and the detector tower can be manually moved in $\mathrm{x}$-direction on an aluminum profile. 
(a)

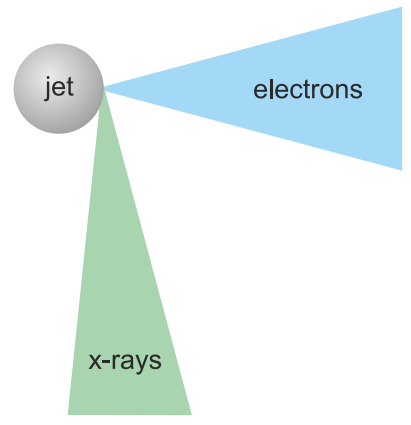

(b)

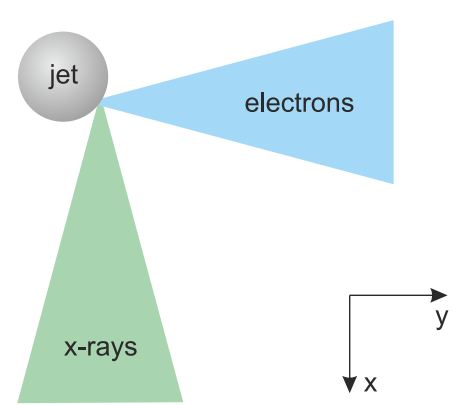

Figure 4.4: $X$-ray generation on a liquid-metal jet. (a) The electron beam hits the center of the metal jet so that the $x$-ray source size in $y$-direction, which is limited by the penetration depth in the metal, is smallest. (b) The electron beam is positioned off the center to reduce self-absorption, resulting in a larger spot size in $y$-direction.

generation, an asymmetric electron spot-size is used, where the size in $x$-direction is typically four times larger than the size in $z$-direction. As the emitted x-rays form an angle of $90^{\circ}$ with the electron beam, the effective x-ray spot size behind the exit window mainly depends on the electron-beam size in $z$-direction and the penetration depth in the liquid-metal jet limits the size in $y$-direction. Typical operation parameters of the source are an electron spot of $16 \times 4 \mathrm{\mu m}^{2}(\mathrm{~h} \times \mathrm{v})$ with an electron-beam power of $40 \mathrm{~W}$. The electron spot is positioned $25 \mu \mathrm{m}$ off the jet center to reduce self-absorption in the metal and to yield a relative symmetric x-ray spot. With these settings, measurements of a test pattern at $E_{\max }=70 \mathrm{kVp}$ show that $2 \mu \mathrm{m}$ vertical lines and spaces (limited by the source size in $y$-direction) and $3 \mu \mathrm{m}$ horizontal lines and spaces (limited by the source size in $z$-direction) can be resolved. Thus, the effective x-ray spot size is estimated to be around $4 \times 6 \mathrm{\mu m}^{2}$ $(\mathrm{h} \times \mathrm{v})$.

The metal used for the source is an alloy consisting of about $70 \%$ gallium, $20 \%$ indium and $10 \%$ tin (Galinstan), with its main x-ray energies at $9.25 \mathrm{keV}$ ( $\mathrm{Ga} \mathrm{K}-\alpha$ ) and $24.2 \mathrm{keV}$ (In $\mathrm{K}-\alpha$ ), resulting in a typical energy spectrum shown in Fig 4.5(a). For measurements of thick specimens, the energy of the gallium line may be too low and absorbing foils can be used to block this main energy in order to use the indium line. As every metal foil reduces the total flux, the absorber should be as thin as possible while removing the unwanted energies. Nickel has an absorption edge at $8.3 \mathrm{keV}$. Thus, it is well suited to block the gallium $\mathrm{K}-\alpha$ line. However, as the bremsspektrum slightly below $8.3 \mathrm{keV}$ also has a large part of the spectrum, 

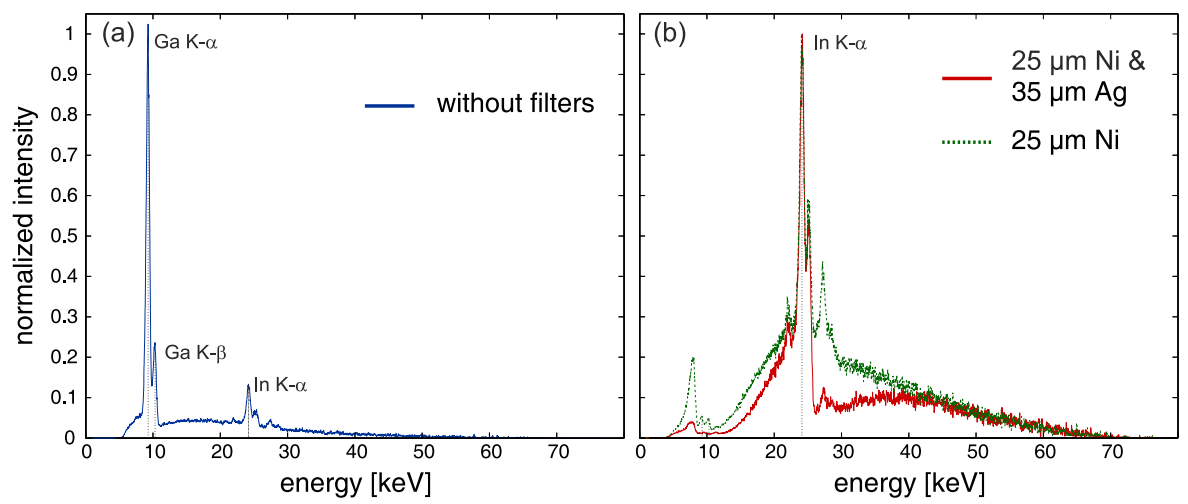

Figure 4.5: Influence of absorption filters to the energy spectrum. (a) shows the pure spectrum of the liquid-metal jet source JXS D2 with an indium rich Galinstan alloy obtained at $70 \mathrm{kVp}$ at a distance of $x_{02}=25 \mathrm{~cm}$ to the source. In order to block the low energies, absorbing foils can be inserted. (b) shows the spectra measured with the same detector at the same position where a 25 $\mu \mathrm{m}$ thick nickel foil has been inserted (green dashed line) and in presence of two foils: $25 \mu \mathrm{m}$ nickel and $35 \mu \mathrm{m}$ silver (red line). All spectra are corrected for transmission of the $0.38 \mathrm{~mm}$ thick silicon chip and normalized to their peak intensities to enable the comparison.

a low energy peak at around $8 \mathrm{keV}$ can still be present. Figure 4.5(b) shows the spectrum measured at a typical sample position of $x=25 \mathrm{~cm}$, where first a 25 $\mu \mathrm{m}$ nickel foil blocks low energies and second, the $25 \mu \mathrm{m}$ nickel foil together with a $35 \mu \mathrm{m}$ silver foil absorb them. Obviously, the nickel foil alone is not enough to block the low energies sufficiently and a more monochromatic spectrum is achieved using both foils. Current developments of the metal-jet source are aiming at using different alloys that have higher indium content [98]. However, these alloys are not liquid at room-temperature anymore and they need to be preheated to operate the source, which poses new challenges to the technical implementation.

\subsection{Using synchrotron-radiation}

\subsubsection{KB based setups at ID22 \& ID16a}

In an ellipse the distance from one focus to the other is always the same, if the path involves a reflection at the ellipse boundary. Thus, if an elliptical mirror is built such that one focus lies in the x-ray source spot and the other some short 


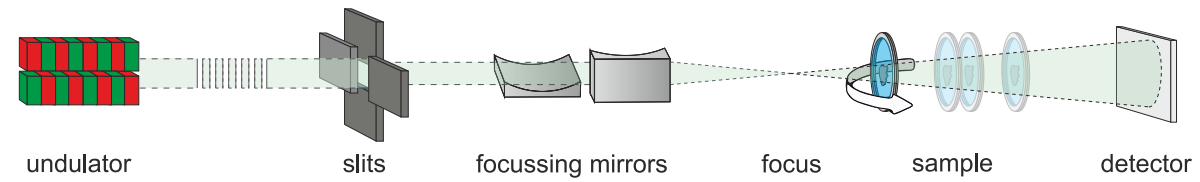

Figure 4.6: Sketch of a KB based setup like those at ID16a and ID22: X-rays are generated in an undulator. After a certain distance, a part of the beam is selected by a pair of slits and focused to a sub-100 $\mathrm{nm}$ spot that determines the resolution for the imaging experiments. A motorized sample tower allows positioning of specimens in the defocused beam and a rotation stage can be used for tomographic measurements.

distance behind the mirror, every photon reflected at this mirror will constructively interfere in the focus behind the mirror. This principle is used to achieve focusing in one direction. A second mirror with a different focal length can be placed before or after the first mirror to enable $2 \mathrm{D}$ focusing. If mirrors are built in such a way, one speaks of Kirkpatrick-Baez (KB) mirrors.

Synchrotron radiation created in an undulator offers a very low divergence (about 10 prad) and a relatively small source size down to some micrometer at new best facilities. Hence, if absorption in air can be avoided, e.g., by using an evacuated flight tube, large propagation distances are enabled to increase the spatial coherence. The generic idea of a KB-based beamline-setup for propagation-based phase-contrast is sketched in Fig. 4.6. At some distance behind the undulator a pair of beam-defining slits is placed, followed by KB-mirrors to focus the radiation to a small spot. The focusing is achieved with KB-mirrors that are coated with a multi-layer structure and bent with 4 actuators to an elliptical form [106]. A secondary source is created $27 \mathrm{~m}$ behind the undulator by using high heat-load slits. After another $36 \mathrm{~m}$ the KB mirrors are located, which allow a minimal focus size below $(60 \mathrm{~nm})^{2}[12]$. The size of the focus limits the resolution achievable in imaging experiments. A fully motorized sample tower enables the alignment of a rotation axis in the beam. After some propagation distance the radiation is detected by a high-resolution detector, which is ideally placed such that the primary beam fills out the whole field of view.

For experiments relevant for this thesis, the beamlines ID16a and ID22 at the European synchrotron radiation facility (ESRF) were used for phase-contrast imaging with a divergent KB-beam. At both beamlines, a lens-coupled x-ray detector is chosen, where a thin single crystal scintillator is viewed by an optical microscope and the image is captured with a fast readout and low noise (Frelon) camera 


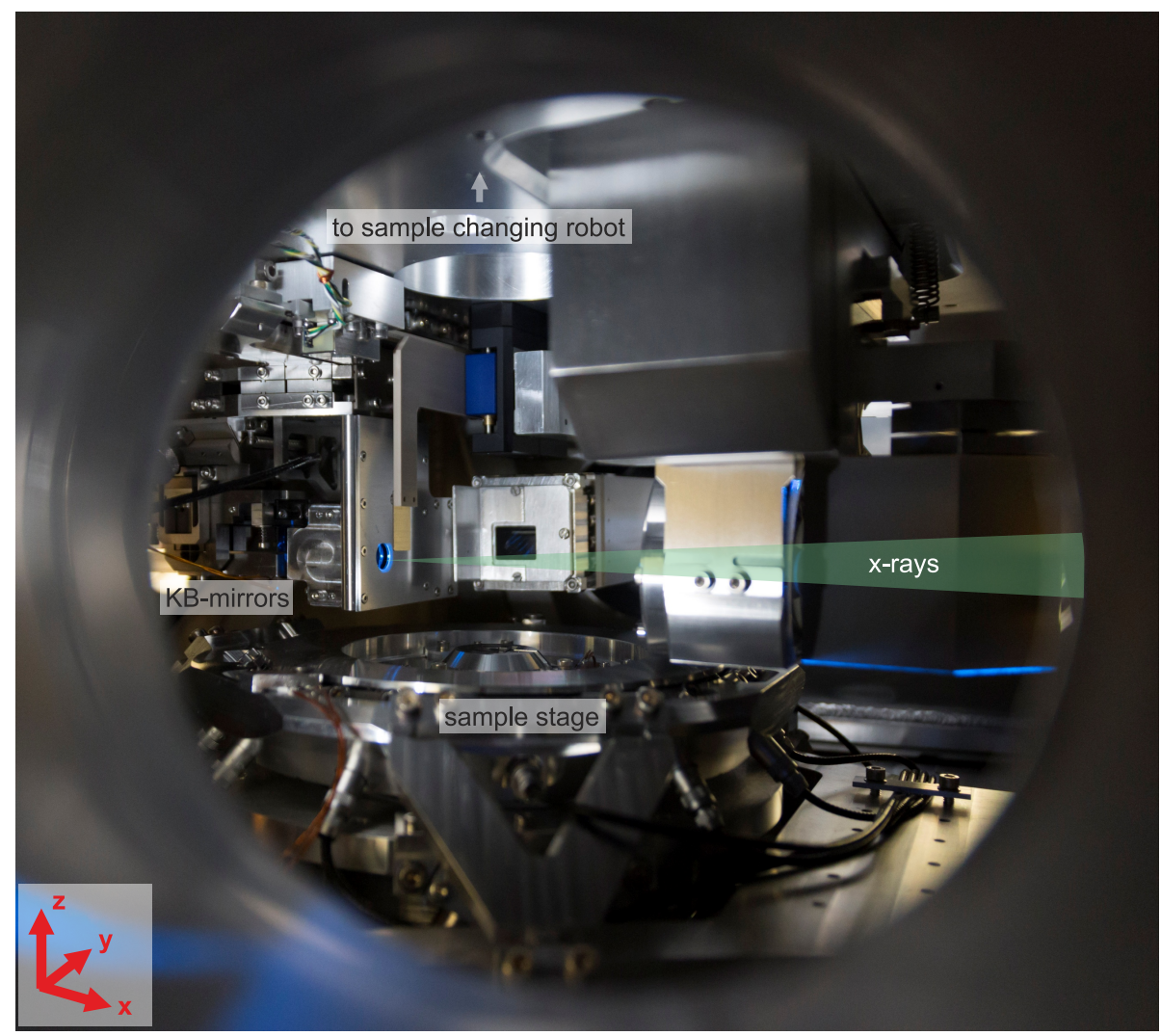

Figure 4.7: Photograph of the sample chamber at the ID16a beamline. The $\mathrm{x}$-ray beam (coming from left) is focused by a pair of KB mirrors to a nanoscale spot. A robot delivers samples from above that are clamped in the rotation stage. The rotation stage can be aligned using a hexapod and translated along the defocused beam. The picture was taken through a vacuum view port, during a sample change. Photograph courtesy of Johannes Hagemann.

[95]. For the ID22 beamline, the chosen optics lead to a detector pixel size of $p=0.756 \mu \mathrm{m}$. The detector was placed at a distance to the focus of $x_{02}=526 \mathrm{~mm}$ where it was fully illuminated by the focused x-ray beam. The sample stage consists of an air-bearing rotation stage, whose pitch and roll angle can be tilted and that can be aligned at different positions in the defocused x-ray beam. Above the rotation, a small motor can be moved in, which is able to push the sample in $y$ direction, to allow the centering of small objects or regions of interest. The energy of the radiation is set to $E=17.5 \mathrm{keV}$, where the bandwidth of $\Delta \lambda / \lambda=0.015$ is determined by the multilayer mirrors. 
At the ID16a, the KB mirrors are located at about $185 \mathrm{~m}$ behind the undulator and by using a fixed-curvature, multi-layer KB-mirror, focus sizes in the range of 10 to $20 \mathrm{~nm}$ in both directions are expected in the future $[44,96,106]$. The energy is fixed at $E=17.0 \mathrm{keV}$ with a bandwidth similar to those at the ID22 beamline. All components are in a vacuum chamber that in the future will allow the handling of cryogenic samples. A photograph of the setup is shown in Fig. 4.7. Samples are delivered by a sample changing robot that transfers samples from a vacuum gate to the ultra-high-vacuum chamber. The robot pushes a standard sample pin in a hole in the center of the rotation. The rotation axis can be aligned using a parallel kinematic robot (hexapod) underneath the rotational positioner and can be moved along the optical axis, to allow measurements in the defocused x-ray beam. The lens-coupled detector is positioned outside the vacuum chamber at a distance to the focus of $x_{02}=337.26 \mathrm{~mm}$. The choice of optics in the detector results in a pixel size of $p=0.845 \mu \mathrm{m}$.

\subsubsection{The empty-beam problem}

Figure 4.8(a) shows a typical empty image $I_{0}$, obtained at the ID22 beamline. High-frequency artifacts are present in the illumination as a result of imperfections and figure errors of the focusing KB-mirrors. A polystyrene sphere of $10 \mu \mathrm{m}$ diameter was placed at $z_{01}=8.8 \mathrm{~mm}$ behind the focus, resulting in an effective pixel size of $\Delta x_{\mathrm{eff}}=12.6 \mathrm{~nm}$. By free-space propagation, a holographic image of the sphere is formed, from which the measured raw data $I_{\text {raw }}$ is shown in Fig. 4.8(b). Obviously the structure of the empty beam dominates the image. The
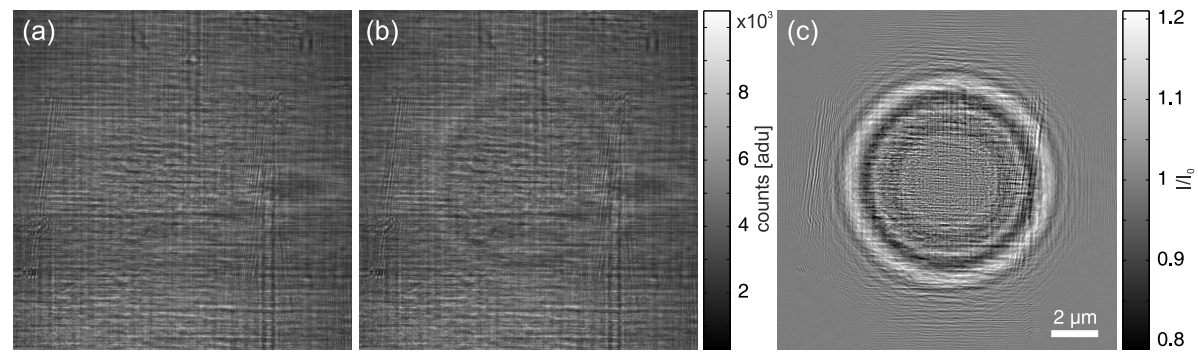

Figure 4.8: The empty-beam problem: (a) shows a part of a typical emptyimage of the primary KB-beam. Many high-frequency artifacts are present due to imperfections of the mirror surfaces. (b) shows a raw hologram of a $10 \mu \mathrm{m}$ polystyrene sphere, obtained with the illumination shown in (a). (c) Is the result after dark-field and empty-beam correction. Figure based on [65]. 
conventional approach to obtain the hologram of the sample is to perform an empty-beam division. As the camera may have a fixed noise pattern, also a darkfield correction is applied. The correction can thus be written as

$$
I_{\text {corr }}=\frac{I_{\text {raw }}-I_{\text {dark }}}{I_{0}-I_{\text {dark }}}
$$

where $I_{\text {dark }}$ denotes the dark-field of the detector. This correction is applied to the measured images and the resulting normalized hologram is shown in Fig. 4.8(c). It can be seen that in regions where no object was present the division yields a uniform field. However, the hologram of the sample is heavily disturbed by the illumination at regions where the sphere was present. To understand the formation of these artifacts, we consider the measured image, which can be written as

$$
I_{\text {raw }}\left(\mathbf{r}_{\perp}\right)=\left|\mathcal{D}_{z}\left(O\left(\mathbf{r}_{\perp}\right) \cdot P\left(\mathbf{r}_{\perp}\right)\right)\right|^{2}
$$

with the object transmission function $O\left(\mathbf{r}_{\perp}\right)($ Eq. (1.60)) and the illumination $P\left(\mathbf{r}_{\perp}\right)$. The empty-beam measurement yields

$$
I_{0}\left(\mathbf{r}_{\perp}\right)=\left|\mathcal{D}_{z}\left(P\left(\mathbf{r}_{\perp}\right)\right)\right|^{2}
$$

For the following we omit writing down the $\mathbf{r}_{\perp}$ dependence. Assume we have a perfect detector without fixed pattern dark field, i.e., $I_{\text {dark }}=0$, and the emptybeam correction is performed

$$
I_{\text {corr }}=\frac{I_{\text {raw }}}{I_{0}}=\frac{\left|\mathcal{D}_{z}(O \cdot P)\right|^{2}}{\left|\mathcal{D}_{z}(P)\right|^{2}} .
$$

If the propagated product $\mathcal{D}_{z}(O \cdot P)$ can be decomposed in the propagated object and probe, i.e.

$$
\mathcal{D}_{z}(O \cdot P) \stackrel{!}{=} \mathcal{D}_{z}(O) \cdot \mathcal{D}_{z}(P)
$$

Eq. (4.7) reads

$$
I_{\text {corr }}=\frac{\left|\mathcal{D}_{z}(O \cdot P)\right|^{2}}{\left|\mathcal{D}_{z}(P)\right|^{2}}=\left|\mathcal{D}_{z}(O)\right|^{2} .
$$

Hence, the correction yields the modulus squared of the propagated object, as intended. However, the assumption defined by Eq. (4.8) is often not fulfilled, as demonstrated experimentally in Fig. 4.8. Instead, the complex wave field at the position of the sample has to be reconstructed for both the empty-beam and the beam with object inside. If both wave fields are known, they can be 
propagated back and the division can be performed in the object plane, where the $\mathcal{D}_{z}$ operator is equal to unity. In practice, it is quite difficult to reconstruct the pure empty-beam without artifacts, even if multiple distance measurements are used [65]. A different way of treating the empty-beam problem is to use a nearfield ptychography approach, where the object $O\left(\mathbf{r}_{\perp}\right)$ and the probe $P\left(\mathbf{r}_{\perp}\right)$ are reconstructed simultaneously using additional constraints, e.g., by using lateral and/or longitudinal shifts of the object [146, 165].

It is instructive to discuss the validity of Eq. (4.8). For the case of the illumination being a parabolic wave, we have already shown in section 1.3.4 that the propagated field can be written as a product of the propagated illumination and the propagated object, in other words, Eq. (4.8) holds true. Recently a mathematical validity criterion for the empty-beam correction has been derived that is presented in [76]. Especially high-frequency variations in the illumination will lead to large errors if small Fresnel numbers, e.g., due to large propagation distances, are present. However, if the illumination consists of mainly low frequencies, as it is the case for a perfect spherical or Gaussian illumination, Eq. (4.8) will be a good approximation. A way to reduce high-frequency variations in the illumination is to low-pass filter the illumination, e.g., by placing a small pinhole in the focal plane [14].

\subsubsection{X-ray waveguides}

The best low-pass filtering of the illumination is achieved for very small pinholes, e.g., with diameters below $100 \mathrm{~nm}$. However, an ideal pinhole to low-pass filter the illumination does not exist. Typical values for the flux densities in the focal plane are $10^{11}$ to $10^{12}$ photons per second. Hence, the pinhole should be thick enough, to have a transmission of about $10^{-10}$ in the blocking regions. To achieve such a transmission for a typical energy of $E=17.5 \mathrm{keV}$, about $15 \mathrm{~mm}$ of silicon or a $100 \mu \mathrm{m}$ thick platinum foil would be necessary [71]. For a diameter of less than $100 \mathrm{~nm}$, a wave propagating through such a cavity will be reflected at the edges several times and in fact the pinhole would be a waveguide. To allow the total reflection of x-rays, the cladding material has to have a smaller refractive index than the guiding core. This is contrary to the case of visible light and is caused by the fact that the refractive index for x-rays is smaller than one.

The use of x-ray waveguides to filter the radiation has many advantages, i.e., the radiation can be focused to spots below $10 \mathrm{~nm}$ [92] and spatial coherence is increased [127]. Figure 4.9 shows two realization principles of $2 \mathrm{D}$ x-ray waveguides. The first, shown in (a) and (b), is based on using two crossed one-dimensional 
(a)

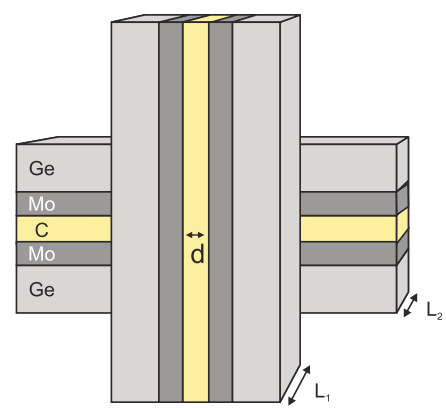

(c)

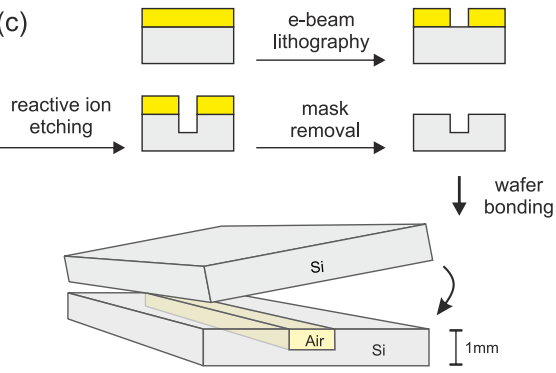

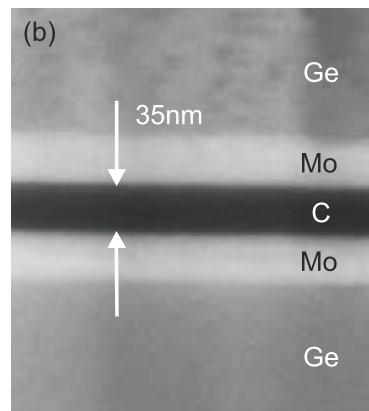

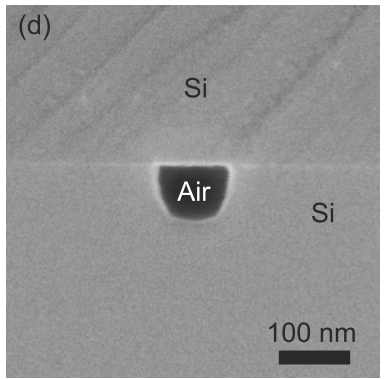

Figure 4.9: $X$-ray waveguides used to optimize the illumination for propagationbased phase-contrast imaging. (a) shows a sketch of a crossed multilayer waveguide, with the carbon guiding layer $(C)$ and additional molybdenum cladding (Mo). (b) shows a scanning electron micrograph of the entrance of a multilayer waveguide. (c) shows the fabrication scheme for bonded waveguides, where silicon is used as cladding and the wave will be guided in air and (d) shows an electron micrograph of the entrance of a bonded waveguide with about $90 \times 70$ $\mathrm{nm}^{2}$ channel dimensions. Figures based on [91, 120].

waveguides. A one dimensional waveguide can be produced by sputtering different materials on a substrate. For this thesis, waveguides with an optimized design of the refractive index profile are used [91]. To this end, a germanium wafer is used as a substrate, where a molybdenum cladding and a carbon guiding layer are sputtered onto, followed by a second cladding layer and a final germanium coating as sketched in Fig. 4.9(a). A second germanium wafer is bonded on top of the multilayers to increase the thickness, such that the primary beam can be blocked. With such a structure very large aspect ratios of the guiding channel are possible, e.g., a guiding layer with $d=9 \mathrm{~nm}$ thickness with a length of about $L_{1}=0.5 \mathrm{~mm}$ [92]. However, as the carbon layer will also absorb the radiation, the total transmission of this kind of waveguides is limited.

Figure 4.10 shows a typical KB far-field (a), obtained at the GINIX setup, which is 

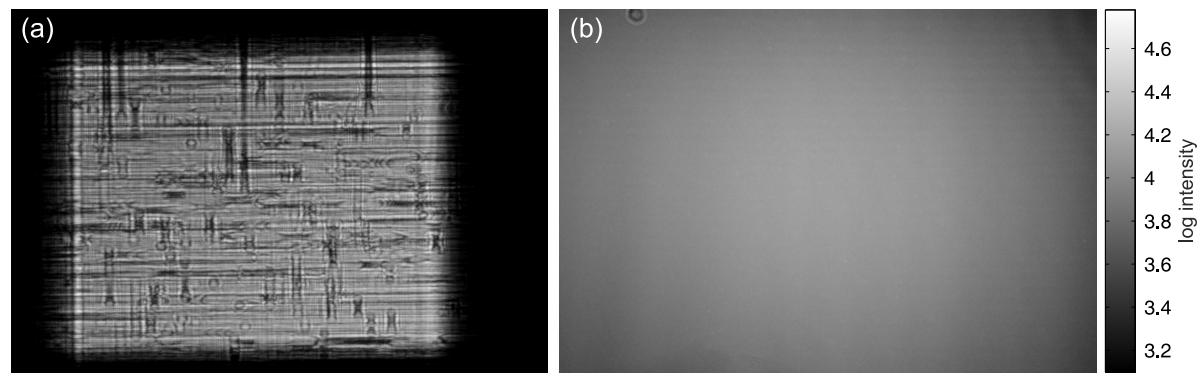

Figure 4.10: Comparison between $K B$ and waveguide illumination. (a) shows the logarithmic intensity of a KB-beam far-field obtained with the GINIX setup. Typical mirror-roughness artifacts and other aberrations can be identified. (b) shows a measurement of the same area where an x-ray waveguide was precisely aligned in the $K B$ focus, yielding a much smoother illumination. To enable comparability with (b), the intensity in (a) was experimentally scaled down by a factor of $3.7 \cdot 10^{3}$.

described in the next section, in comparison to a waveguide far-field (b), obtained with a crossed-multilayer waveguide with $d=59 \mathrm{~nm}$ guiding layer over a total length of $L_{1}+L_{2}=764 \mu \mathrm{m}$. It can be clearly seen that the typical high-frequency artifacts, which are produced by the already very low surface roughness ${ }^{1}$ of the mirrors, are suppressed very well. Additionally, the smaller source size can be seen as an increased divergence. Near-field reconstructions of far-fields obtained from this waveguide ${ }^{2}$ yield a source size of $16 \times 13 \mathrm{~nm}$ [88], confirming the finite difference simulations carried out for the optical design. However, due to the geometric acceptance and also due to the limited transmission of the waveguide, more than three orders of magnitude in intensity are lost. Typical flux densities behind a crossed multi-layer waveguide are in the range of $10^{8}$ photons per second for an energy of $13.6 \mathrm{keV}$.

Another approach to fabricate x-ray waveguides is based on air-filled guiding channels in silicon. This will allow higher transmission rates and the waveguides can be produced with arbitrary length. Figure 4.9 (c) shows a sketch of the fabrication principle, which is similar to chip fabrication in information technology. A silicon wafer is coated with a resist that will change upon electron impact. With an electron-beam lithography setup comprising an interferometric sample stage,

1 Typical roughness values of KB-mirrors are about $1 \mathrm{~nm}$ peak to valley over about $15 \mathrm{~cm}$ total length of the mirror.

2 Note that a far-field as shown in Fig. 4.10(b) is not suitable for a nearfield reconstruction, as many features of the illumination are not in the field of view. Instead, a far-field obtained with a Pilatus detector is used, see e.g., [14]. 
structures with large aspect ratios can be written. The mask is developed and etched in the silicon wafer. After mask removal, a second wafer can be bonded on top, to obtain a closed channel [120]. A scanning electron micrograph of a final x-ray waveguide is shown in Fig. 4.9(d). This waveguide has a size of $90 \times 70 \mathrm{~nm}^{2}$ which yields a source size of about $26 \mathrm{~nm}$ in both simulations and nearfield reconstructions [18]. Additionally, the transmission especially at low energies is more than one order of magnitude higher compared to the crossed multilayer waveguides. Typical flux values are in the order of $10^{9}$ photons per second at $7.9 \mathrm{keV}$. This approach also enables the production of more sophisticated designs, e.g., using a tapered geometry [33]. Here, the entrance will be larger to allow the collection of more photons and the width will gradually decrease during propagation in the channel. Using this approach, up to $10^{10}$ photons per second could recently be measured at the GINIX setup.

\subsubsection{Waveguide based setup GINIX}

The Göttingen instrument for nanoscale imaging with x-rays (GINIX) is an experimental setup that can be installed at the P10 beamline of the Deutsche Eletrektronen Synchrotron (DESY) in Hamburg. The initial instrumentation and commissioning was realized as a part of the doctoral thesis of Sebastian Kalbfleisch in 2012 [85] and is dedicated to several applications requiring a nano-focused x-ray beam [154]. A major part of the present work was the improvement of the setup to enable phase-contrast tomography using x-ray waveguide optics at nanoscale resolution. The generic setup, sketched in Fig. 4.11, is very similar to the KB-based setups present at ID22 and ID16a.

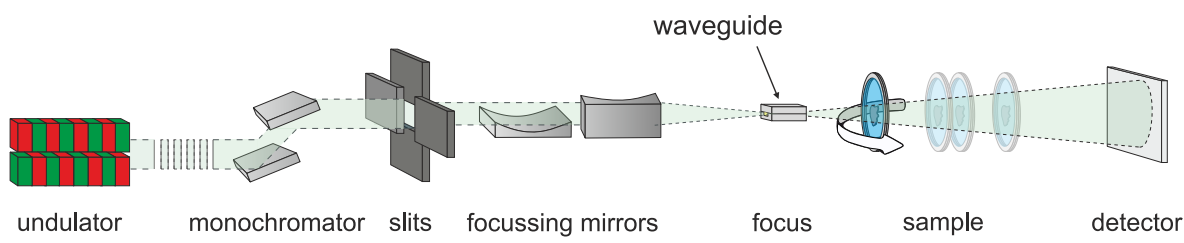

Figure 4.11: Sketch of a waveguide based setup at GINIX. X-rays are created by accelerating electrons in an undulator. After some propagation distance, the $\mathrm{x}$-rays are monochromatized by Bragg reflection at two silicon crystals. After about 90 meters, the x-rays are focused with KB-mirrors to a sub-micrometer spot in which an x-ray waveguide is precisely aligned. Behind the waveguide a sample can be positioned and rotated for tomographic experiments. 
With its huge circumference of $2304 \mathrm{~m}$, the storage ring PETRA III of DESY is currently the most brilliant synchrotron-radiation source world-wide [49]. The coherence beamline P10 has different instruments that can be switched, depending on the application. After the radiation is created in an undulator, it is monochromatized by a high heat-load double-crystal $\mathrm{Si}(111)$ monochromator that limits the bandwidth of the x-rays to about $\Delta \lambda / \lambda=10^{-4}$. A beam-position monitor is installed to correct motions of the monochromator that are caused by thermal effects. The main part of the GINIX instrument consists of an optical table that houses a vacuum chamber with a pair of KB-mirrors installed, followed by several motorized positioners for additional optics and a motorized sample tower, which includes an air-bearing rotation. About $84 \mathrm{~m}$ behind the undulator, the beam size is defined by a pair of slits and at a distance of about 87 meters, the KB-mirrors are placed to focus the radiation to a spot size below $400 \mathrm{~nm} \times 200 \mathrm{~nm}$. The energy range, in which best performance of the mirrors is achieved, is between $E_{\min }=6 \mathrm{keV}$ and $E_{\max }=15 \mathrm{keV}$.

Figure 4.12 shows a photograph of the latest configuration of the experimental setup. Behind the KB-box very small positioners (SLC-1720, SmarAct, Germany) are present to allow alignment of an aperture. This $1 \mathrm{~mm} \times 1 \mathrm{~mm}$ pinhole is used to only transmit the double-reflected beam that will yield the focus about 20 $\mathrm{cm}$ behind the last mirror. A parallel kinematic robot in a hexapod configuration (SmarPod 110.45, SmarAct, Germany) serves as a positioner for the x-ray waveguides. The SmarPod allows moving the waveguide in all six degrees of freedom with $200 \mathrm{~nm}$ repeatability for the translations and $10 \mu \mathrm{rad}$ for rotations, each for the full travel range [162]. For smaller travel ranges the accuracy is even higher. This allows precise alignment of the waveguide in the KB-focus. The SmarPod is hold via kinematic bases (KB1X1/M, Thorlabs, Germany) to allow easy removal of the whole positioner with better than $30 \mu \mathrm{rad}$ angular and $30 \mu \mathrm{m}$ lateral repeatability. The top plate of the hexapod is also equipped with a kinematic base, where the waveguides are placed onto. Figure 4.13 shows a close up of the sample region, where a bonded waveguide can be seen. The high repeatability of the kinematic bases allows the preparation of mounted waveguides prior to the experiment to facilitate alignment during the beamtime.

Behind the waveguide, a sample is positioned on a motorized sample tower that is able to move an air bearing rotation in all three directions. A flat, self-built positioner allows the alignment of pitch and roll angle $(\theta$ and $\phi)$ of the rotation stage. Above the rotation, $\mathrm{x}$ - and $\mathrm{y}$ - movements are enabled with a specially designed SmarAct positioner that has a travel range of about $40 \mathrm{~mm}$ with sub- 


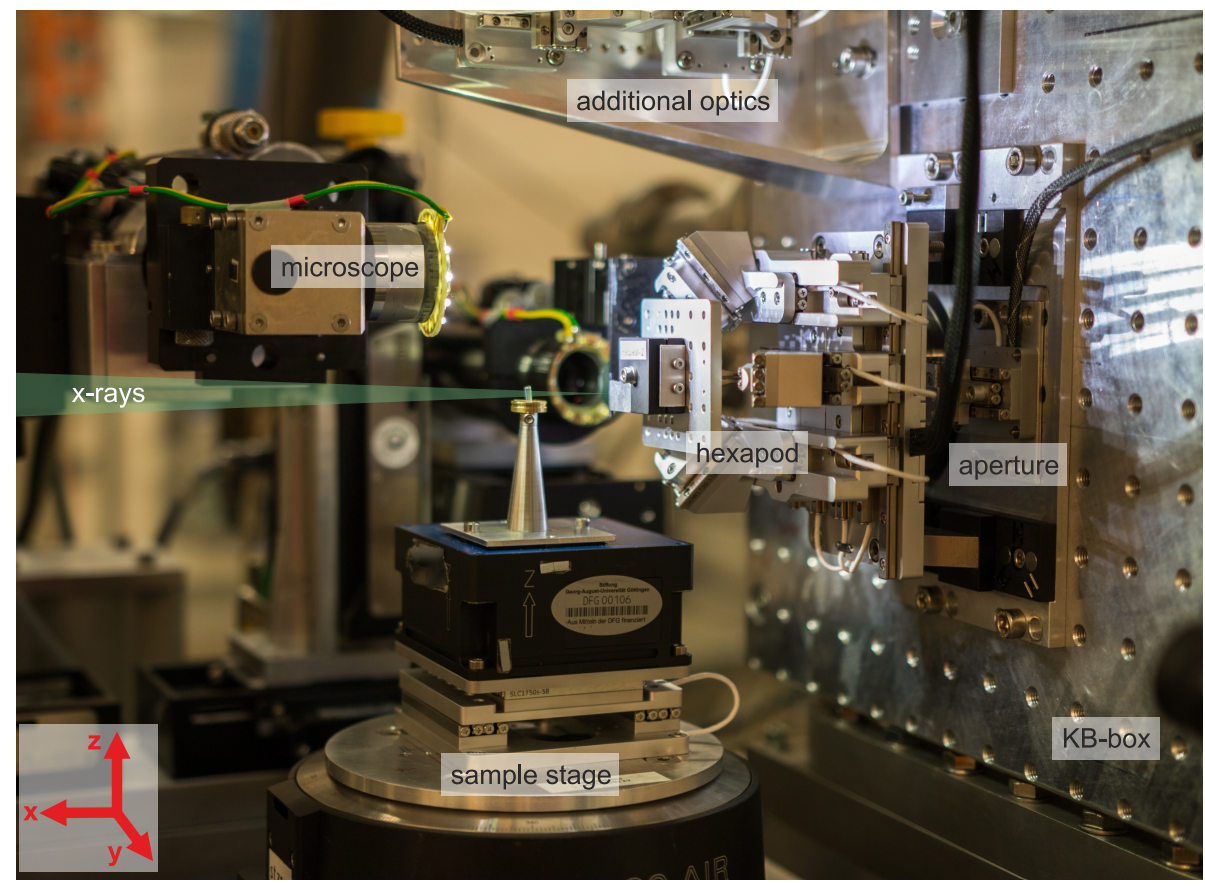

Figure 4.12: The GINIX end station at the P10 beamline. X-rays (coming from right) are prefocused by KB-mirrors that are located in the evacuated KB-box. $A$ motorized aperture allows select the reflected beam and to block the direct beam. A hexapod is used to align a second optic, here a bonded waveguide, in all degrees of freedom in the focus of the KB-beam. Behind the waveguide, the motorized sample tower with rotations and translations positions a specimen that can be viewed by an on axis microscope. A holder for additional optics can be seen at the top that in the setup is tilted away by $90^{\circ}$.

nanometer scan accuracy. On top of this, a piezo cube (Physik Instrumente, Germany) can be used for fast-scanning experiments. A key point to achieve nanoscale 3D reconstructions are cables above the rotation axis. The friction between moving cables and other positioners may lead to significant disturbances of the rotation axis or the sample. To avoid any unwanted motions, all cables above the rotation are fit through an aperture in it.

The last part visible in Fig. 4.12 and 4.13 is an on-axis-view (OAV) microscope that has a drilled hole to allow the transmission of the direct beam. The microscope is equipped with a network camera (Manta GigE camera, Allied Vision, USA). Thus, real time microscopic observation of the sample is enabled during the $\mathrm{x}$ ray measurements. This facilitates alignment of small samples and enables the 


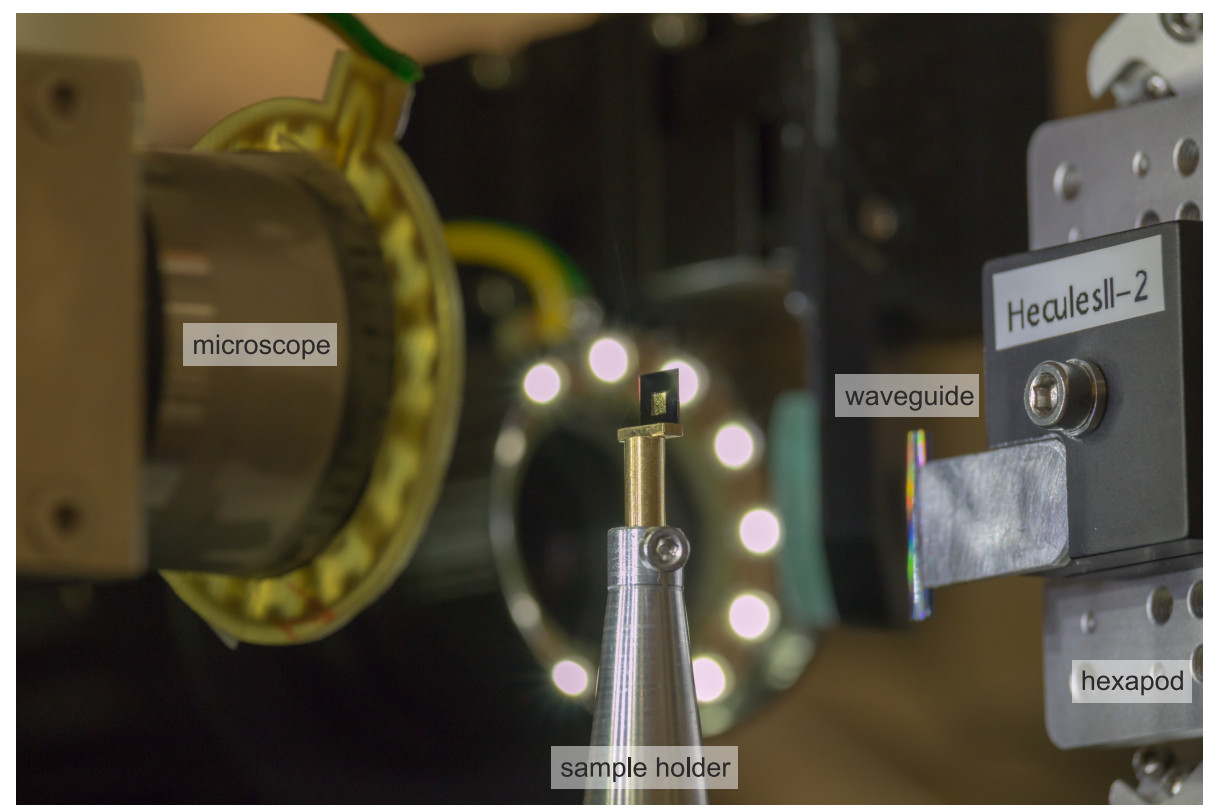

Figure 4.13: Close up photograph of a the GINIX end station showing (from right to left): a bonded waveguide mounted on the hexapod, the sample holder, holding a $\mathrm{Si}_{3} \mathrm{~N}_{4}$ window with dried cells on it and the on-axis microscope. Note that in this picture the waveguide was moved out of the $\mathrm{x}$-ray beam .

observation of radiation damage or movements of the samples.

As the x-ray waveguides provide a very small effective source size and an increased numerical aperture, a very high resolution is possible. Using an optimized experimental setup, where vibrations between the waveguide and the sample holder are minimized, we could recently demonstrate a resolution below $30 \mathrm{~nm}$ using the HoloTIE approach [88] and by using additional constraints in iterative phase-retrieval, ${ }^{3}$ a resolution of about $23 \mathrm{~nm}$ could be achieved on solid-state test-structures [16].

3 In this particular example, a compact support, vanishing absorption and negativity of the phase shift are used as constraints. 


\section{Tomographic imaging of the mouse lung}

To understand the fundamental basics of many diseases and to find suitable treatments, animal experiments are a very important tool. Especially small animals like mice or rats, are easy to breed and are well established. The conventional approach to obtain information about fine details on the cellular level is histology, where thin tissue slices are investigated under a microscope. By contrast, x-rays with their large penetration depth offer a great opportunity to obtain information of the inner organs without the need for slicing and staining. However, currently available CT systems have a limited resolution that is not high enough to resolve fine details or single cells as the contrast fades out for small structures. With the invention of highly brilliant x-ray sources, phase-contrast measurements are enabled that allow the increase of contrast at high resolution.

In this thesis, we will focus on the application of phase-contrast tomography techniques to lung imaging, which is an important tool for medical research. The

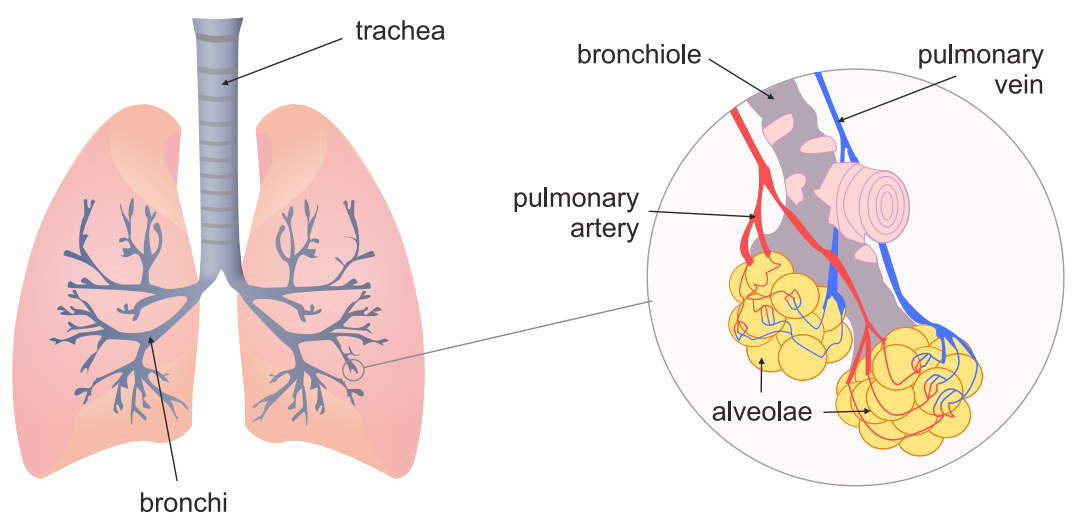

Figure 5.1: Sketch of the anatomy of the lung: Air that is breathed in travels through the trachea that branches into two main bronchi. The bronchi further branch off into smaller bronchioles until they finally end in the alveolae, where the oxygen exchange in arteries and veins takes place. Drawing based on [145]. 
lung is the most important organ for air breathing vertebrates as it enables the oxygen exchange between the respired air and the blood in the body. The general anatomy of the lung is sketched in Fig. 5.1. The air is transported through the trachea that branches off into many bronchi and bronchioles, which finally end in the alveolae. Thus, the cell surface is effectively increased by a large factor to enable the fast oxygen exchange with the blood in a capillary network. The inner surface of the bronchial lumen consists of goblet cells, which can produce mucus to eliminate foreign material from the lung, e.g., dust or pathogenic germs. Additionally, many cilia are present that serve to slowly transport the particles away to finally be coughed up. A major lung-disease is asthma, the cause and progression of which is still not fully understood to date [105]. About ten percent of children and five percent of adults in the western population suffer from asthma. The disease expresses itself as a chronic inflammation of the respiratory tract with a swollen mucosa that is more sensitive to stimuli. As a consequence, recurrent breathlessness and coughing may occur, which can be exacerbated under stress..

To gain a better understanding of the fundamental cellular processes during asthma, its intricate $3 \mathrm{D}$ structure can be accessed at very high resolutions using propagationbased phase-contrast tomography. This and the following chapters will deal with the application of zoom tomography, to observe structures in the lung on very different length scales, ranging from the full organ in whole mice down to single cells that can be detected in relatively large tissues. To this end, healthy and asthmatic mice are the subjects of investigation, which was enabled by collaborations with the group of Frauke Alves at the University Medical Center Göttingen (UMG) and the Max-Planck-Institute for Experimental Medicine Göttingen.

\subsection{State of the art in small-animal imaging}

In the last decade the number of publications using computed tomography for small-animal imaging has risen exponentially [155]. Modern preclinical scanners are equipped with microfocus sources that together with flat-panel detectors allow a resolution down to $50 \mu \mathrm{m}$. Thus, studies of small animals are enabled, e.g., to image primary lung cancers by using contrast agents [8]. However, if no contrast agent is used, the detection of tumors is barely possible in conventional setups and noise dominates the image [86]. The invention of phase-contrast imaging was a key point, to allow the detection of materials with only little difference in absorption like cancer in soft-tissue.

For relatively large objects, the use of the Talbot effect enables phase-contrast 
measurements of weakly absorbing samples using a grating interferometer [179]. Although it was first demonstrated at synchrotron sources, the approach could be translated to the laboratory by using an additional source grating [138]. In addition to the differential phase-contrast, which allows a better sensitivity to weakly absorbing parts, a dark-field contrast can be obtained similarly. It is based on the fact that structures smaller than the resolution element result in small angle scattering, the observation of which allows the direction dependent detection of small features $[137,81]$. However, all grating-based approaches have the drawback that precise matching and alignment of the gratings to each other are necessary. Furthermore, as two additional gratings have to be placed behind the sample, the imaging dose has to be increased. The maximum resolution in phase-contrast measurements is limited to the grating period and to achieve a resolution below $10 \mu \mathrm{m}$, grating-based imaging is not the method of choice [193]. Instead, the use of propagation-based phase-contrast allows the detection of much smaller features, as we will demonstrate in this and the following chapters.

\subsection{Methods}

\subsubsection{Sample preparation}

Whole mice are prepared following the protocol for ex vivo experiments described in [43]. To this end, female inbred mice (BALB/c mice, Harlan Laboratories, USA), are used as a model organism for lung imaging experiments. Healthy mice were sacrificed using an isoflurane overdose. To compensate the collapse of the lung, it is inflated with air using a constant pressure of $30 \mathrm{~cm}$ water column $(2.94 \mathrm{kPa})$. To do so, a series of smaller tubes is used, ending with a PE50 cannula that is fixed inside the trachea with a cotton thread. After inflation the trachea is tied up and the mice are embedded in 1\% low-melting agarose-gel in a $30 \mathrm{ml}$ tube (Fisher Scientific, USA). Before imaging, samples are kept for two hours at $20^{\circ} \mathrm{C}$ to avoid alterations in the following measurements. The preparation is carried out at the UMG in strict accordance with the guidelines for the care and use of laboratory animals of the local ethics office of the UMG and all efforts are made to minimize suffering. As no fixation procedures are used for this preparation, samples are brought to the institute for x-ray physics directly after their preparation, where experiments are immediately carried out to avoid deterioration of the samples. A photograph of a finished prepared sample, glued to a sample holder, can be seen in Fig. 5.2(b). 


\subsubsection{Experimental setup and measurements}

For the experimental realization, the laboratory instrument JuLiA, described in the previous chapter, is used. Figure 5.2 shows the general setup: Electrons are focused to a small spot on the liquid-metal jet. The exact spot sizes and electron powers differ depending on the geometry and are listed in Tab. 5.1. The jet is positioned $25 \mu \mathrm{m}$ off the center to reduce self-absorption in the metal. The tomographic rotation axis is positioned at a distance $x_{01}$ to the source spot and the active area of the detector has a position of $x_{02}=x_{01}+x_{12}$. The distance is calibrated with a copper alignment grid. To this end, the grid is scanned in $y$-direction with a positioner above the rotation axis. The resulting images can be correlated to map the pixel shift to the known physical shift and thus a value for the pixel size is obtained. This measurement is repeated for different source to object distances $x_{01}^{i}=x_{01}+\Delta x$ yielding the magnification $M^{i}=x_{02} / x_{01}^{i}$ for different axial shifts $\Delta x$ that are known from the motor movements. For the inverse magnification it is

$$
\frac{1}{M}=\frac{x_{01}^{i}}{x_{02}}=\frac{x_{01}}{x_{02}}+\frac{1}{x_{02}} \Delta x
$$

Thus, a linear regression of the inverse magnifications depending on the axial shifts $\Delta x$ can be performed to yield $x_{01}$ and $x_{02}$ with a high accuracy down to $20 \mu \mathrm{m}$. Before the distance calibration is performed, pitch and roll angle $(\theta$ and $\phi)$ of the tomographic axis are aligned according to the descriptions in section 3.3. The grid

(a)

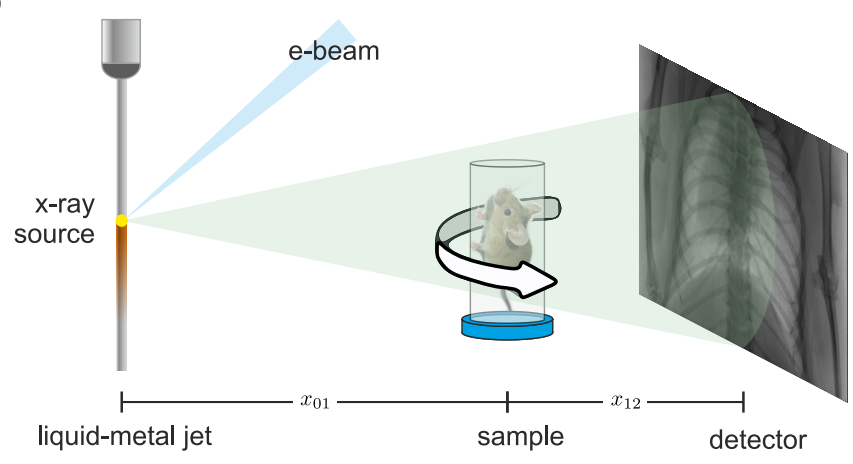

(b)

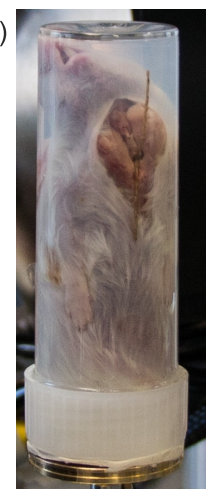

Figure 5.2: (a) Sketch of the experimental setup, showing the principle of the $x$ ray generation with a liquid-metal jet and the used geometry. (b) A photograph of a prepared mouse sample that is used for the experiments. 


\begin{tabular}{lllll}
\hline & large FOV & zoom-move & zoom-detector 1 & zoom-detector 2 \\
\hline$x_{01}$ & $0.24 \mathrm{~m}$ & $0.15 \mathrm{~m}$ & $0.15 \mathrm{~m}$ & $0.15 \mathrm{~m}$ \\
$x_{02}$ & $0.44 \mathrm{~m}$ & $1.53 \mathrm{~m}$ & $0.38 \mathrm{~m}$ & $0.37 \mathrm{~m}$ \\
pixel size $p$ & $55.0 \mu \mathrm{m}$ & $55.0 \mu \mathrm{m}$ & $13.1 \mu \mathrm{m}^{*}$ & $13.1 \mathrm{\mu m}^{*}$ \\
voxel size & $29.9 \mu \mathrm{m}$ & $5.38 \mu \mathrm{m}$ & $5.16 \mu \mathrm{m}$ & $5.26 \mu \mathrm{m}$ \\
$F^{10}$ & $1.2 \cdot 10^{4}$ & 159 & 55 & 57 \\
angular steps & 916 & 995 & 916 & 991 \\
angular range & $183^{\circ}$ & $360^{\circ}$ & $183^{\circ}$ & $360^{\circ}$ \\
exposure time & $0.8 \mathrm{~s}$ & $10 \mathrm{~s}$ & $10 \mathrm{~s}$ & $10 \mathrm{~s}$ \\
source size & $(40 \times 10) \mathrm{\mu m}^{2}$ & $(40 \times 10) \mu \mathrm{m}^{2}$ & $(18.2 \times 5.6) \mathrm{\mu m}^{2}$ & $(20 \times 5) \mu^{2}$ \\
x-ray power & $100 \mathrm{~W}$ & $100 \mathrm{~W}$ & $40 \mathrm{~W}$ & $40 \mathrm{~W}$ \\
total dose & $7.2 \mathrm{~Gy}$ & $246.0 \mathrm{~Gy}$ & $90.6 \mathrm{~Gy}$ & $26.4 \mathrm{~Gy}$ \\
filters used & $\mathrm{Ni}$ & $\mathrm{Ni}$ & $\mathrm{Ni}$ & $\mathrm{Ni}+\mathrm{Ag}$ \\
\hline
\end{tabular}

Table 5.1: Overview of experimental parameters: Exposure time denotes the time for each projection. Dose values are calculated for a model protein $\left(\rho_{m}=\right.$ $1.35 \mathrm{~g} / \mathrm{cm}^{2}$ ) with weighted spectral contributions. ${ }^{*}$ : The detector used for the zoom-detector setting was the Pirra with a pixel size of $6.54 \mu \mathrm{m}$, but images were resampled by a factor of 2 . Used filters are a $25 \mu \mathrm{m}$ thick nickel foil (Ni) and a $35 \mu \mathrm{m}$ thick silver foil (Ag).

is subsequently centered in the rotation axis, such that the calculated distances correspond to those of the rotation-axis center. Additionally, it is ensured that the $y$-movement is carried out perpendicular to the optical axis by zeroing the tomographic axis as described in section 3.3. All alignment procedures are done one day before the sample is received, to ensure a timely measurement. For the tomographic experiments, the samples are centered in the rotation axis and moved in $z$-direction, so that the lung covers the FOV. Before and after a tomographic scan, empty-beam images are recorded by moving the sample out of the beam in $y$-direction. Every raw projection is then dark-field and empty-beam corrected according to Eq. (4.7) to remove residual beam inhomogeneities and non-uniform detector flat-fields.

Tomographic measurements are performed in three different geometric realizations. The first one is chosen to achieve a medium resolution overview scan with about $30 \mu \mathrm{m}$ voxel size, denoted as "large FOV" in the following. A second scan with a higher resolution of about $5 \mu \mathrm{m}$ is performed, either by moving the detector further away, which increases the geometric magnification, denoted as "zoom-move", or by choosing a detector with a smaller pixel size, denoted as "zoom-detector". Table 5.1 shows experimental parameters for each configuration. The zoom-detector setting is further separated based on the used energy filter. 

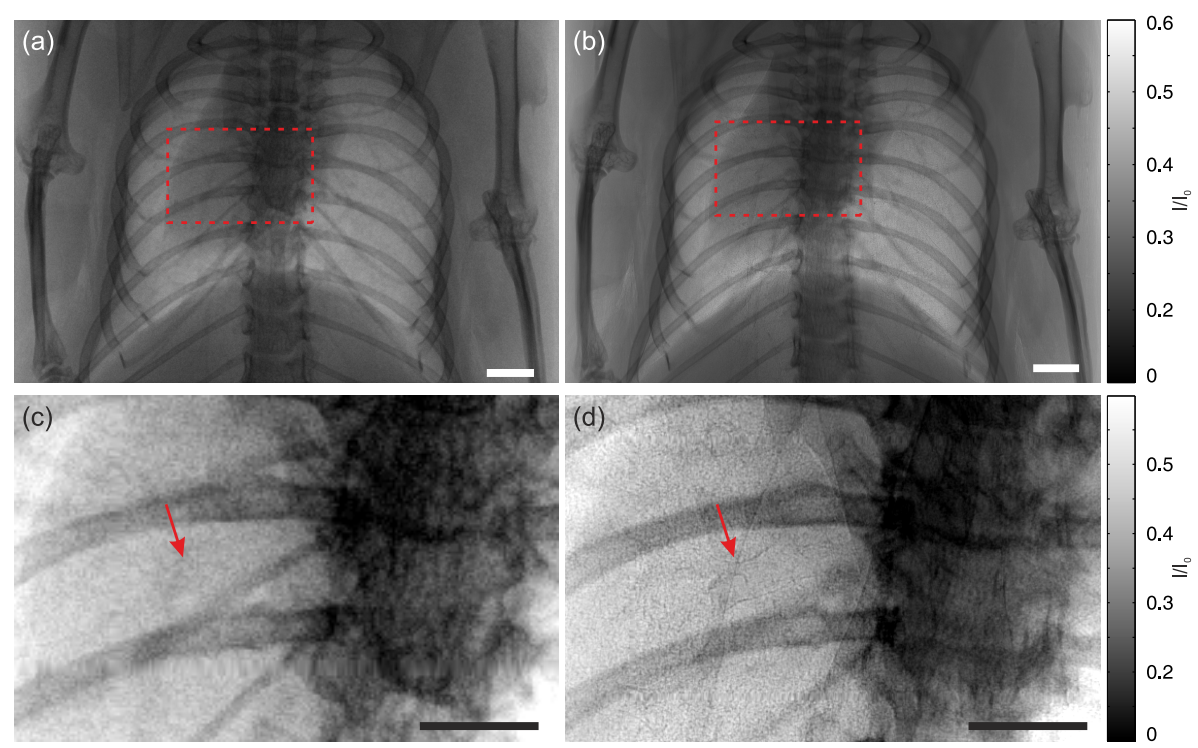

Figure 5.3: Measured absorption (left) and phase-contrast (right) projections of the same mouse: (a) shows a single measurement recorded in the large FOV setting. The marked region is shown as a magnification in (c). (b) shows a measurement of the same projection, but recorded in the zoom-move setting with a smaller pixel size. The sample is scanned laterally to obtain a similar FOV. The same region, marked by the dashed rectangle is magnified in (d), where phase contrast can be observed, in particular at the position of the red arrow. Scale bars represent $2 \mathrm{~mm}$.

The zoom-detector measurements are carried out with the "Pirra" camera (Photonic Science, United Kingdom), i.e., a fiber-coupled sCMOS camera with a 15 $\mu \mathrm{m}$ thick gadox scintillator deposited on top (see Tab. 4.1). As the PSF of the detector is larger than a single pixel, images are resampled such that $2 \times 2$ pixels form a new one, resulting in a detector pixel-size of $13.1 \mu \mathrm{m}$. For the large-FOV and zoom-move setting, the single-photon counting timepix detector is used (XIE, Freiburg, Germany). Besides the fact that the PSF of this detector is a single pixel, the measurements of the empty-beam image can be used to calculate precise dose-values. To this end, the total flux is determined by an empty-beam measurement and corrected by the silicon absorption and air transmission values. Together with the measured energy spectra (see Fig. 4.5) an energy-weighted dose can be calculated for each measurement by using Eq. (1.92). The necessary absorption coefficient is calculated for a model protein with the chemical formula $\mathrm{H}_{50} \mathrm{C}_{30} \mathrm{~N}_{9} \mathrm{O}_{10} \mathrm{~S}_{1}$ and a mass density $\rho_{m}=1.35 \mathrm{~g} / \mathrm{cm}^{3}$. The resulting values are 
listed in Tab. 5.1.

In a conventional micro-CT, the sample is moved very close to the source to achieve high resolution. By contrast, we choose a relatively large source to object distance of $x_{01}=150 \mathrm{~mm}$ as the liquid-metal jet source offers a flux density that is still high enough to allow measurements in a reasonable time. This choice increases the coherence at the sample and the detector has to be moved farther away to achieve a small pixel size. As a result, the effective propagation distance $x_{\text {eff }}=x_{12} / M$ is so large that free-space propagation leads to phase-contrast effects in the intensity distribution. In particular for high-resolution measurements, images will be in the direct contrast regime as indicated by the Fresnel numbers $F^{10}$. Figure 5.3 shows a comparison of an absorption measurement, carried out in the large FOV setting, and a high resolution measurement in the zoom-move setting. In the magnified and contrast adjusted views in the lower row, significant edge-enhancement effects can be observed in the high-resolution measurement (d), which are present mostly at the edges of air-filled bronchial tubes (see the red arrow).

\subsection{Results}

\subsubsection{Phase-contrast in mouse lungs with laboratory sources}

Cone-beam reconstruction software based on an implementation of the FDK algorithm (Ultra-Fast CBCT Reconstruction Software, Bronnikov Algorithms, The Netherlands) is used to obtain 3D reconstructions of the measured projections. To enable fast reconstructions, this software uses integer values for its calculations. To this end, projection values are scaled several times with unknown scale-factors that may change, depending on the dataset. In principle, quantitative values can be obtained from the data by using a different software or normalization procedures. However, as in interior tomography the quantitative values also may change (cf. section 3.2.3), we refrain from stating quantitative density values or Hounsfield units.

Figure 5.4 shows two resulting orthoslices that are oriented perpendicular to the rotation axis. Both measurements are obtained in the large-FOV setting, where in (b) a slightly smaller pixel size of $p=24.8 \mu \mathrm{m}$ was used by moving the detector to $x_{02}=532 \mathrm{~mm}$. As darker values correspond to a higher optical density, bones (black) and air (white) can easily be distinguished from water and soft-tissue. The slices show a cut through a central region of the lung, where cross sections of the ribs surround the lung. The most outer bones correspond to the forelegs. The 
artifacts at the forelegs in (b) are present because not every projection contained information about them due to the smaller FOV. Both reconstructions also show streak-artifacts caused by beam-hardening, which are particularly visible at the bones.

A small part, marked by dashed red rectangles in Fig. 5.4, indicates the regions of interest that are used for local tomography at higher resolution. For (a) the zoomed measurement is performed by moving the detector further away (zoommove), whereas for (b) the zoom is carried out by changing the detector and the distance $x_{01}$ (zoom-detector configuration). As already indicated in Fig. 5.3, the measurements at higher magnification will contain edge-enhancement due to phase-contrast effects. To obtain quantitative projection values, phase retrieval using the SMO algorithm is performed prior to the tomographic reconstruction according to Eq. (2.15). The regularizing parameter $\kappa=\delta / \beta$ is chosen to $\kappa=530$ by visual inspection. Notice that the use of the BAC algorithm did not result in a higher resolution here, as residual motion artifacts disturb high resolution

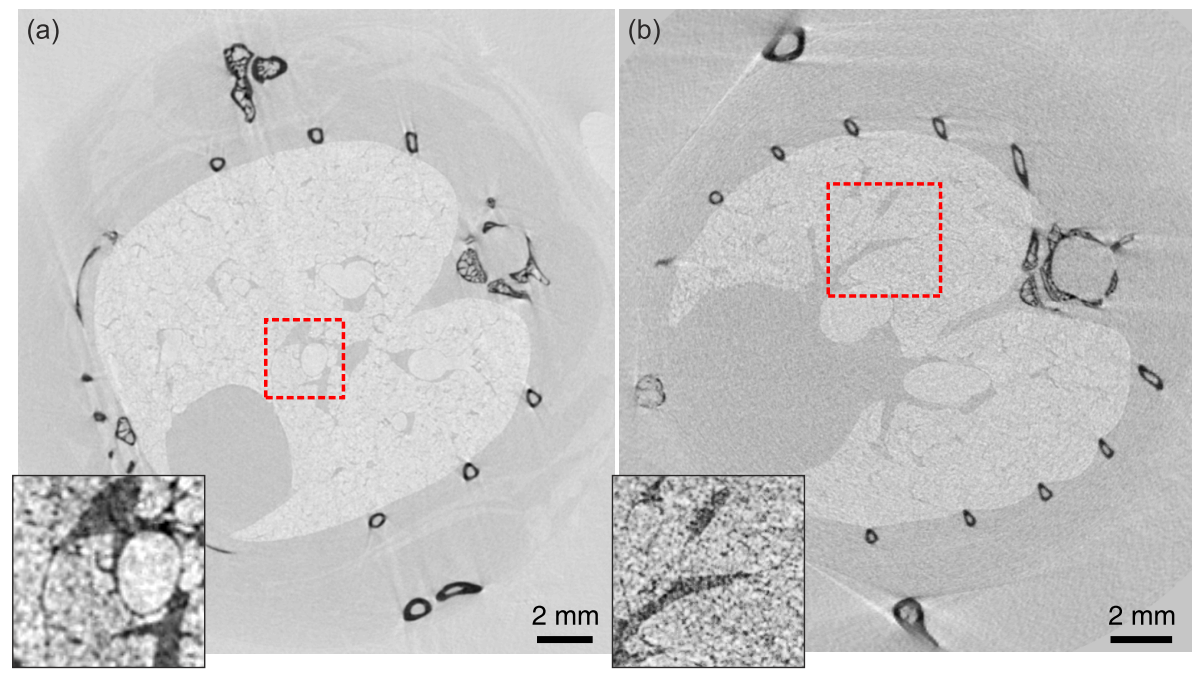

Figure 5.4: Orthoslices through the tomographic reconstruction of two different mice recorded in the large-FOV setting. In (b) a slightly smaller pixel size of $p=25 \mu \mathrm{m}$ is chosen by moving the detector closer to the sample. Darker values correspond to a higher optical density, so the lungs appear brighter than the surrounding soft tissue and water. The regions marked by the dashed rectangles are shown as magnifications in the insets. The same regions are used for measurements at higher resolutions in Fig. 5.5. 
information. Instead, spurious streak artifacts could be observed in the orthoslices if the BAC algorithm is utilized. After phase retrieval, additional ring-removal based on the Fourier-filter approach is performed as described in section 3.2.1.

Figure 5.5 shows the influence of phase retrieval on the reconstructed slices in the zoom-detector $(a, b)$ and the zoom-move setting $(c, d)$. The values along a profile of 6 pixels width through the red lines in (c) and (d) are plotted in (e) and (f) to provide information about the quantitativeness of the reconstructed values. While in the reconstructions without prior phase retrieval structures can be seen by eye, a difference between water and air is hardly visible in the profile in (e). The profile through the phase-retrieved values (f) shows a significant difference between water and air. Additionally, a third density value can be observed that is barely visible in (c). With a density between air and water, we attribute these structures to fat that surrounds a blood vessel. The quantitative values enable a density-based segmentation by thresholding the histogram or an automatic volume rendering, where for the latter every density value is mapped to a color and a transparency value.

All reconstructed slices in Fig 5.5 show slight low-frequency variations, which are caused by failures of the ring removal algorithm. Additionally, the slices in Fig. $5.5(\mathrm{a})$ and (b) seem to have a wrong rotation axis. This effect is caused by motion that occurs during the measurement. Especially high resolution information in the slices is altered by these motion artifacts. The reconstructions in Fig. 5.5(c) and (d) show less artifacts. However, if different slices along the optical axis are viewed, blurred and doubled structures can be observed (see Fig. 5.7) that are typical indicators for motion artifacts.

Figure 5.6(a) shows a 3D rendering of the large-FOV dataset, shown in Fig. 5.4(a). To this end, bones (gray) and air (half-transparent rose) are automatically labeled using an histogram-based segmentation, implemented in Avizo Fire 7 (Visualization Sciences Group, FEI Company, USA). Several large bronchi can be observed inside the half-transparent lung. The region where the heart is expected can be recognized in Fig. 5.4(a) as the round hole with a higher density (darker gray) surrounded by the air of the lung. This region is manually segmented and its rendering (red) can be seen behind the bones on the left side in the $3 \mathrm{D}$ rendering in Fig. 5.6(a). The region used for the high resolution measurement (Fig. 5.5(c) and $(d))$ is indicated by a dashed rectangle.

Figure 5.6(b) and (c) show the bones together with a 3D rendering of the highresolution zoom-move dataset from two different viewing angles. For the rendering phase-retrieved slices like those in Fig. 5.5(d) are used and the $3 \mathrm{D}$ volume is 
(a)

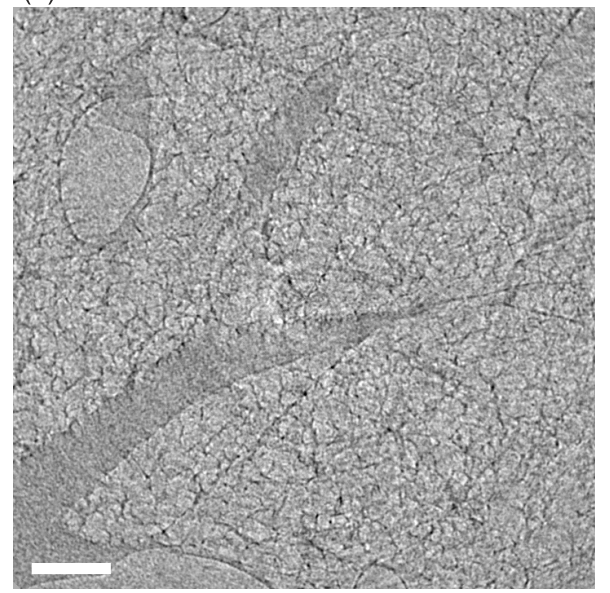

(c)

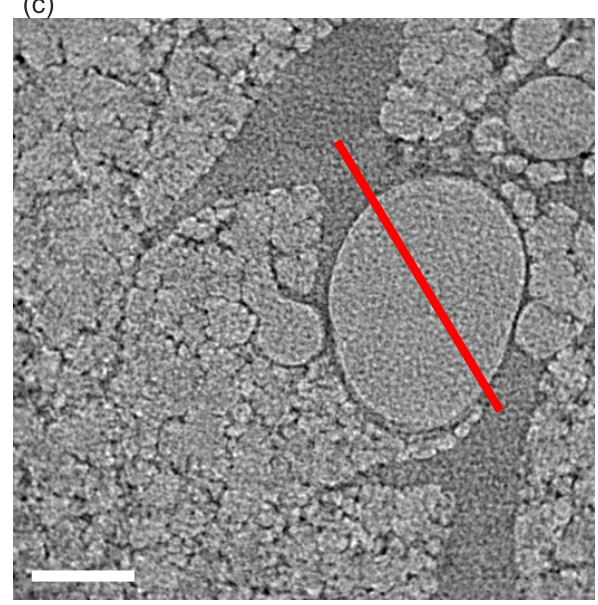

(e)

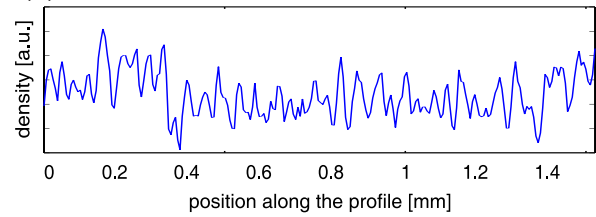

(b)

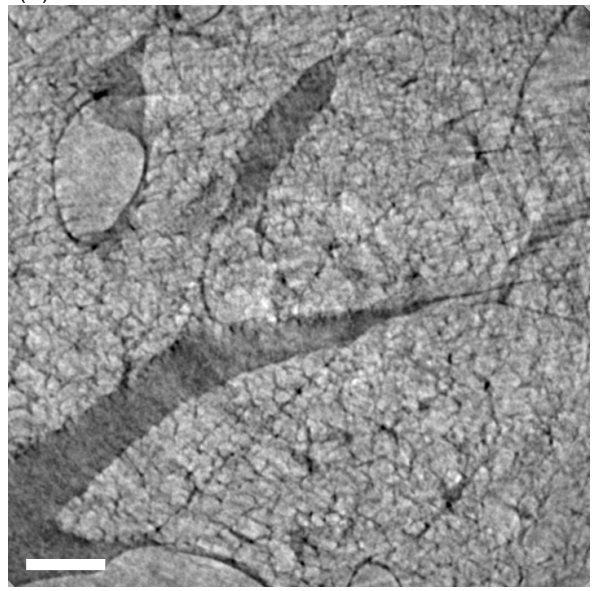

(d)

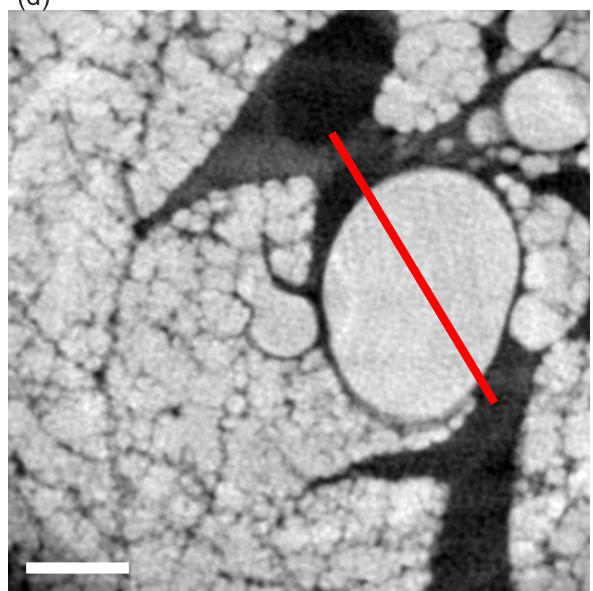

(f)

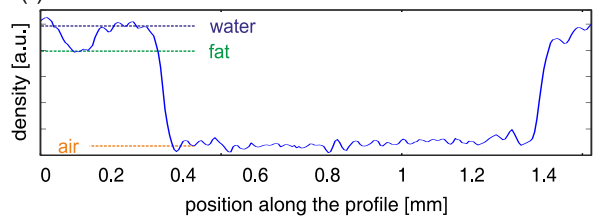

Figure 5.5: Reconstructed high-resolution orthoslices of the regions marked in Fig. 5.4, measured in the $(a, b)$ zoom-detector and $(c, d)$ zoom-move setting. (a) and (c) show reconstructed orthoslices, without any phase-retrieval algorithm applied. (b) and (d) show the same slices but with SMO phase-retrieval performed prior to the tomographic reconstruction. The reconstructed values along profiles of 6 pixels width (red lines in (c) and (d)) are shown in (e) and (f) for their respective reconstructions above. It is worth mentioning that motion artifacts heavily disturb the high resolution information in the zoom-detector setting in (a) and (b). All scale bars denote $500 \mu \mathrm{m}$. 
(a)
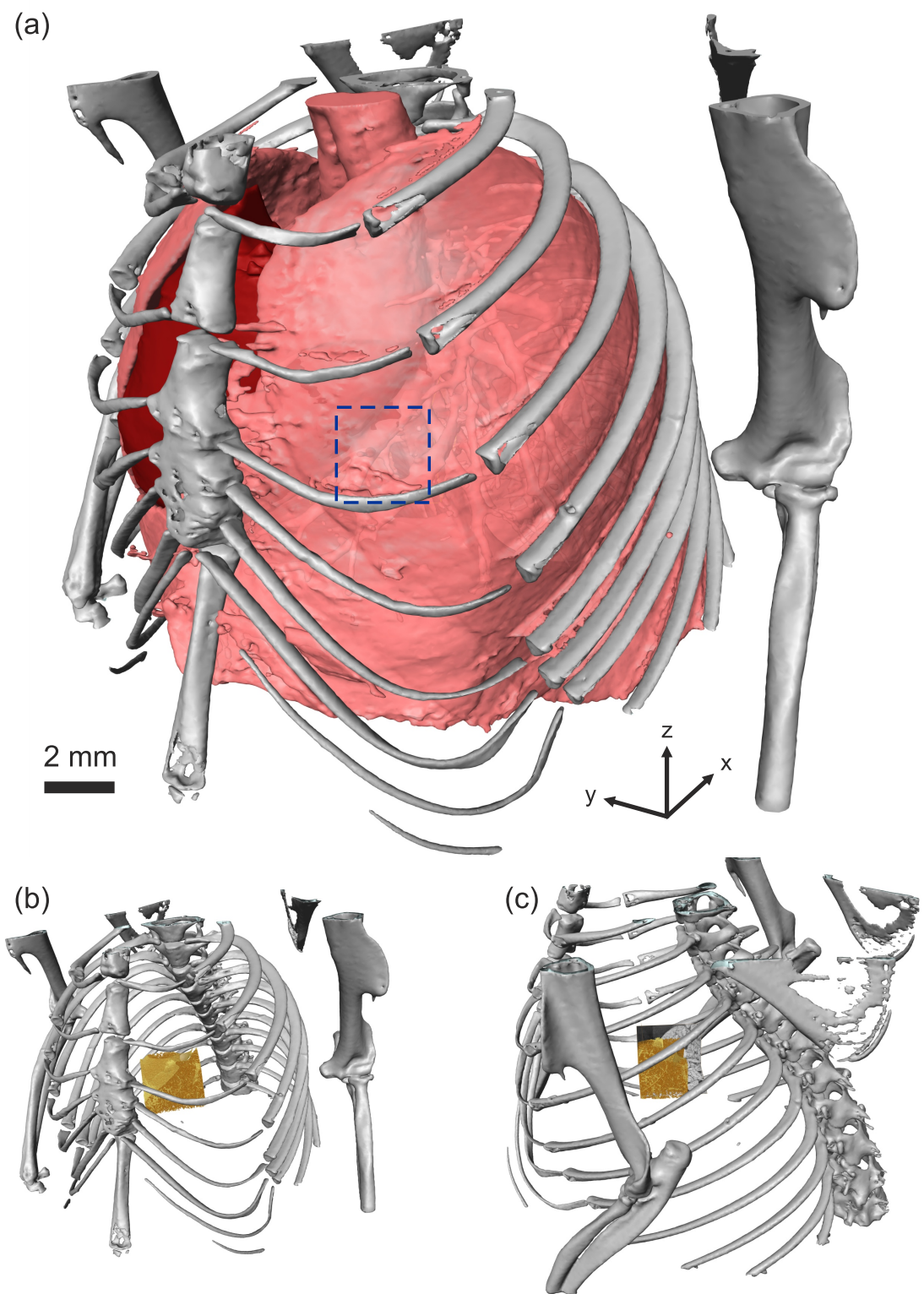

Figure 5.6: (a) 3D Rendering of a reconstructed large-FOV volume. Automatically segmented bones and air are shown in gray and rose, respectively. The region of the heart is manually segmented (red), which can be identified behind the bones on the left side. The dashed rectangle indicates the area measured at higher resolution. (b) and (c) show a rendering of the bones together with the rendering of Fig. 5.7, which was aligned with respect to the large-FOV dataset. 


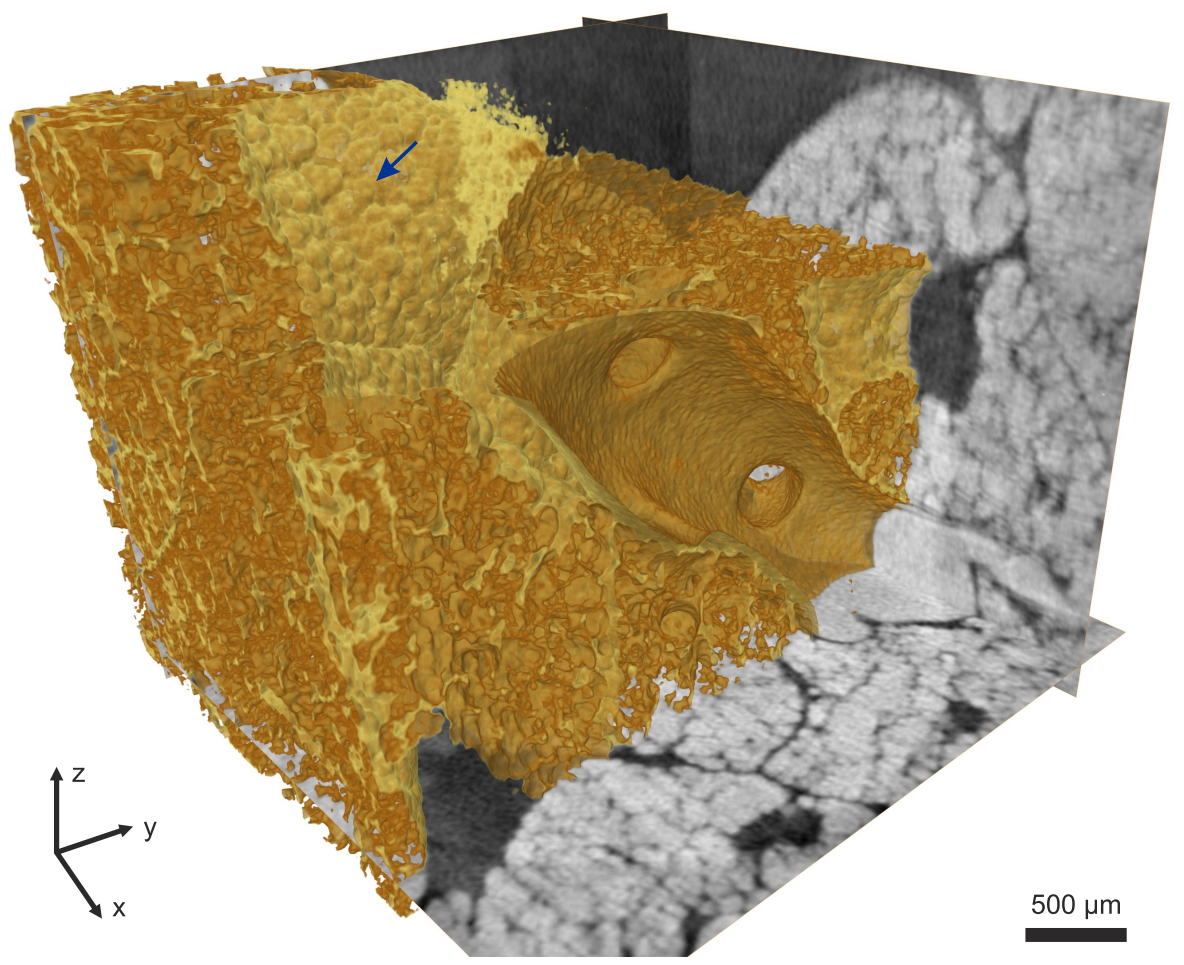

Figure 5.7: 3D Rendering of the phase-retrieved tomographic reconstruction in zoom-move setting (Fig. 5.5). Three orthoslices are shown together with an automatic volume rendering of a sub-volume that shows a cut through a bronchus with several junctions. Single alveoli can be resolved, especially at the position of the blue arrow.

aligned with respect to the larger dataset. Figure 5.7 shows a close up of this high resolution dataset, consisting of three orthoslices together with an automatic volume rendering. The latter is limited to a sub-volume, such that a cut through a bronchus can be seen that shows several junctions to smaller bronchi or bronchioles. The blue arrow marks the position of the blood vessel, visible in the orthoslice in Fig. 5.5(d), which enables the observation of the alveoli surface next to it. Notice that the rendering gives consistent information about the $3 \mathrm{D}$ structure although the slice in the xz-plane is blurred due to the before mentioned motion artifacts. 


\subsubsection{The influence of the energy spectrum}

As discussed in section 4.3, the main energy at the present liquid-metal jet source is the gallium K-alpha line with an energy of $E=9.25 \mathrm{keV}$. A $30 \mathrm{~mm}$ thick water-filled tube would absorb nearly all of the radiation at this low energy. For the imaging of whole mouse samples in $30 \mathrm{~mm}$ diameter tubes, this means that energies at $9.25 \mathrm{keV}$ will not contribute to the image, but be fully absorbed by the sample and would therefore unnecessarily increase the dose. For x-rays with the energy of the indium K-alpha line at about $E=24 \mathrm{keV}, 30 \mathrm{~mm}$ of water still transmit about $25 \%$ of the incoming radiation [71]. Hence, for all measurements shown so far, we tried to block the gallium K-alpha line using a $25 \mathrm{\mu m}$ nickel filter. However, as can be seen in Fig. 4.5(b), a relatively large part of the low energies still remains in the spectrum. For measurements with a smaller $x_{01}$ these will have an even stronger contribution, as air-absorption will be lower. If an additional silver filter is used, a further reduction of low energies can be achieved. The unfiltered photon-energy spectrum of a solid target molybdenum anode, with its main energy at about $E=17.5 \mathrm{keV}$ (Mo K-alpha), was also not sufficient to obtain a high transmission signal.

A third mouse is measured in a configuration with an additional $35 \mu \mathrm{m}$ silver filter. Once again, we first record an overview scan, then choose a region of interest and perform a second scan at this position with a higher resolution. Here, the zoom is enabled by changing the detector (zoom-detector 2), as the Pirra detector not only has smaller, but also more pixels. Images are resampled as before and phase retrieval using the BAC algorithm (Eq. (2.19)) is performed.

Figure 5.8 shows the results obtained in this configuration. An orthoslice of the overview scan that only shows absorption contrast can be seen in Fig. 5.8(a). The dashed rectangle marks the region used for the scan at higher resolution. The inset in Fig. 5.8(a) shows this region as it is obtained from the absorption contrast measurement. Figure 5.8(b) and (c) show orthoslices of the high resolution data, without and with phase retrieval, respectively. As the BAC algorithm (Eq. (2.19)) was employed here, a higher resolution compared to the SMO reconstruction (see section 2.1.3) is enabled. In contrast to the measurements presented in the previous section, the BAC algorithm did not result in any streak artifacts in the tomographic slices.

Compared to the results of the previous chapter, the dataset in Fig. 5.8 has a much higher quality. Beam hardening artifacts can no longer be observed at the bones in the overview scan (a) as was expected with the harder beam. Additionally, 


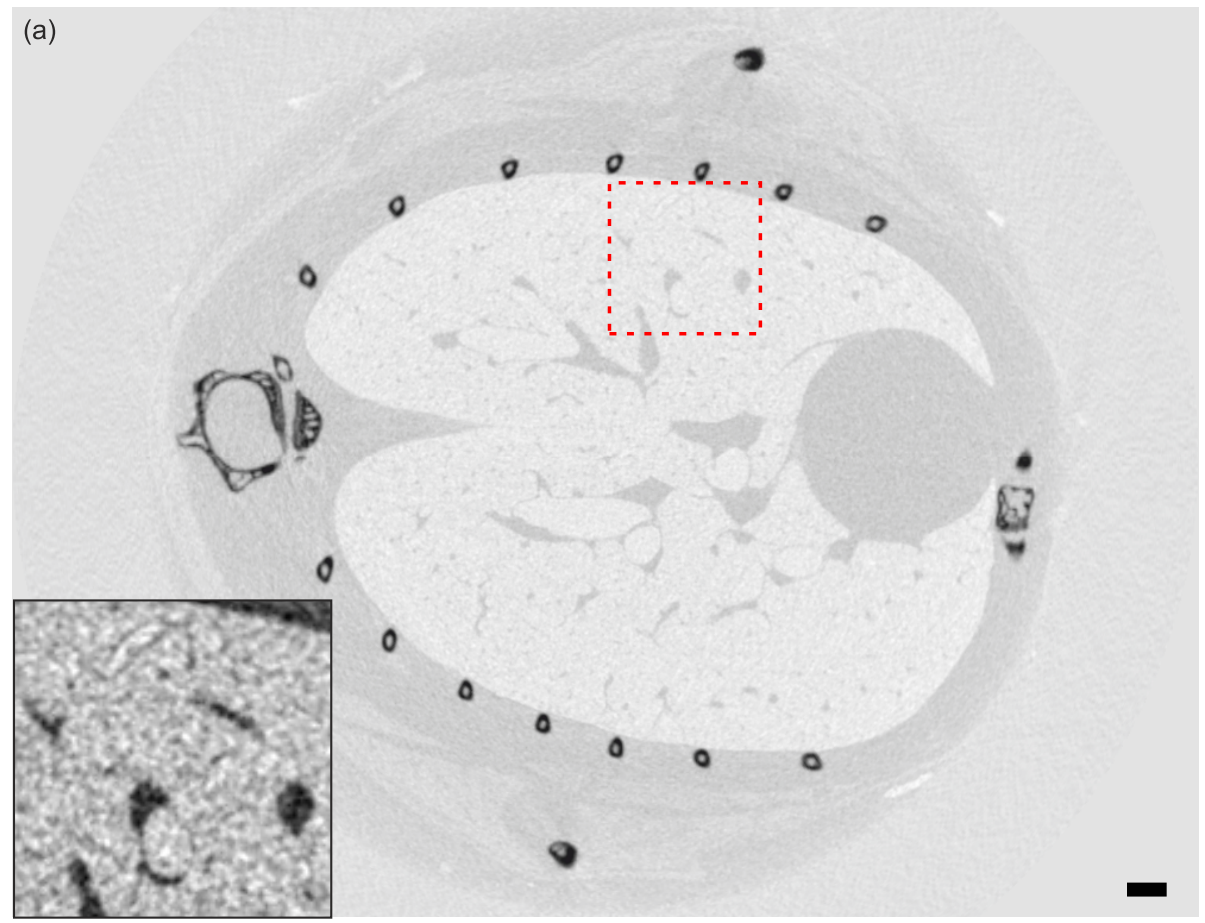

(b)

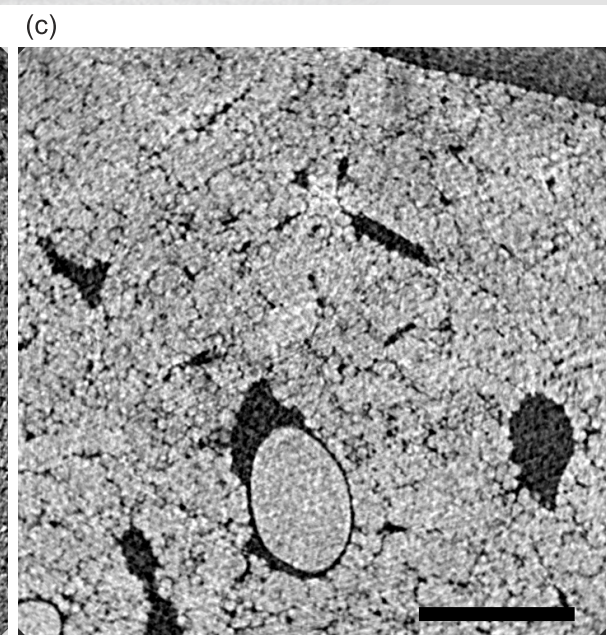

Figure 5.8: Reconstructed orthoslices using an additional silver filter to block low energies more efficiently. (a) shows the overview slice recorded in the largeFOV setting. Beam hardening artifacts at the bones can no longer be observed. The region marked by the dashed rectangle is shown in the inset and used for the high resolution measurement. (b) shows the reconstructed slice in zoomdetector 2 setting without phase retrieval, where for (c) BAC phase-retrieval was applied. In contrast to previous measurements, motion artifacts in the high resolution measurement are successfully avoided. Scale bars denote $1 \mathrm{~mm}$. 
fine structures can be observed in the high resolution scan and it does not show any motion artifacts, which may be one reason for better performance of the BAC algorithm. Due to the use of the silver filter, the dose for this measurement was significantly lower (see Tab. 5.1). Thus, less energy is deposited into the sample and it is less likely that motions are caused by the ionizing radiation. However, due to the lower dose, the signal to noise ratio is lower than in the reconstruction shown in $5.5(\mathrm{~d})$, but it is still high enough to observe fine structures like alveolae.

\subsection{Summary and outlook}

In summary, we have shown the application of propagation-based phase-contrast tomography to whole mice with laboratory sources. A key point to achieve phasecontrast measurements in a reasonable time frame was the use of a liquid-metal jet based x-ray source. However, the current version of the source is optimized for imaging at about $9.25 \mathrm{keV}$, which is too low for relatively thick specimen like a whole mouse. By using energy filters to block the lower energies, high-quality reconstructions could be obtained. The combination of a coarse overview scan with a high-resolution interior scan at a specific region-of-interest allowed observation of fine details of the lung with about $5 \mu \mathrm{m}$ resolution. Phase retrieval suitable for the direct contrast regime could be successfully applied to reconstruct values proportional to the electron density, which enabled the automatic segmentation and $3 \mathrm{D}$ rendering of anatomical structures inside the mouse. By this means, the precise location of regions of interest inside the large volume of the whole mouse becomes possible. Thus, propagation-based phase-contrast tomography offers a huge potential for biomedical research, as high resolution $3 \mathrm{D}$ information can be obtained. It may not only be used for a better understanding of processes involved in asthma, but could also advance knowledge of other diseases, in particular lung cancer, especially if combined with functional contrast agents as we will present later in chapter 7 .

To obtain reconstructed 3D volumes as shown in this chapter, a total exposure time of about 2.5 hours was necessary for the interior measurements. Current developments of new liquid-metal jet sources aim at increasing the high energy content of the x-ray spectrum by using different alloys that need to be preheated [98]. A new prototype source will also offer an additional increase in brilliance by a factor of about 8 to 10 [98].The combination of both source improvements 
will enable similar measurements as those shown here but with a significantly reduced exposure time in the range of a few minutes. Thus, propagation-based phase-contrast measurements on living mice could become possible in the near future. 


\section{Single-cell imaging}

The detailed knowledge of structures at the single-cell and at the sub-cellular level is important to understand fundamental basics relevant for biological and biomedical processes. Microscopy is a key technology and without it, modern science as we know it, would not be possible. The maximum resolution achievable with conventional microscopes is limited by Abbe's resolution criterion to

$$
d=\frac{1.22 \lambda}{2 \cdot N A}
$$

This equation states that two points can be just resolved, if their distance $d$ to each other is larger than about half the wavelength $\lambda$ divided by the numerical aperture $N A=n \cdot \sin \alpha$ with the refractive index $n$ and the half-opening angle $\alpha$. For light with $\lambda=400 \mathrm{~nm}$ and $N A=1$, best optical microscopes can achieve a resolution in the range of $200 \mathrm{~nm}$. In 2014 the Nobel Prize was awarded to Stefan Hell for the invention of optical nanoscopy, which allows fluorescent imaging using visible light at higher resolutions down to $20 \mathrm{~nm}$ [70]. However, if cells are going to be studied in their natural environment, i.e., inside a much larger organ, only surface-near cells can be reached. An alternative approach to increase the resolution is the use of near-field effects such as in scanning tunneling microscopy [21], which is also limited to effects very close to the surface.

X-rays have a high penetration depth and, depending on their energy, can easily pervade many centimeters of bone and soft tissue. As the wavelength of hard x-rays is in the range of about $\lambda=0.1 \mathrm{~nm}=1 \AA$ and below, their possible resolution power seems very high. However, lenses for hard x-rays with a high numerical aperture do not exist to date, so that the resolution is far from $1 \AA$. In this chapter we will apply propagation-based phase-contrast with hard x-rays for single cell imaging at nanoscale resolution. 


\subsection{High resolution imaging of macrophages}

Macrophages are white blood cells (leukocytes), i.e., they are part of the immune system of an organism. They are a special kind of phagocytes, protecting the organism by ingesting harmful pathogens and other foreign objects or material. The uptake of particles is called phagocytosis, for which the cells stretch out their pseudopodia to incorporate particles by endocytosis and store them in vesicles inside the cytoplasm. Phagocytes are important throughout the animal kingdom and are highly developed within vertebrates [40].

Currently, a widely used method to obtain nanoscale-resolution images of biological specimens is electron microscopy. The energy of electrons and thus their wavelength can be easily tuned by accelerating them in electric fields. Magnetic fields can act as lenses that give a high numerical aperture. Alternatively, phaseretrieval approaches like ptychography can be used to avoid aberrations introduced by magnetic lenses [118]. By this means, microscopes can be built that achieve atomic resolution $[38,175]$. The major drawback of electron microscopy is the strong interaction with the samples, which limits the maximum thickness in transmission electron microscopy (TEM) to about $300 \mathrm{~nm}$ or to the surface of structures of interest in scanning electron microscopy (SEM). For TEM, samples have to be cut into ultra-thin slices with only some nanometers thickness. Additionally, tedious sample preparation involving staining with highly toxic materials like osmium tetroxide and uranyl acetate are necessary.
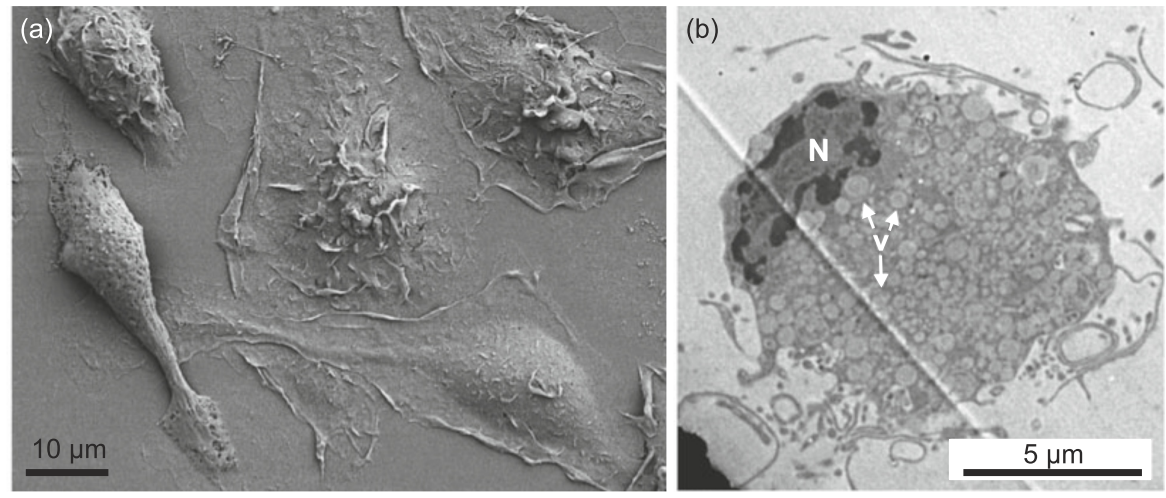

Figure 6.1: (a) SEM image of murine macrophages (b) TEM image of a ultrathin slice of a macrophage. The nucleus (N) can be observed and some vesicles (v) are marked by arrows. Pictures are taken from [149]. 
Figure 6.1 shows two electron microscopy images of specially prepared murine macrophages on a plastic substrate [149]. In the SEM image, shown in (a), several macrophages can be seen. In particular at the lower ones, the process of phagocytosis can be observed, where the pseudopodia (the "arms") are stretched to reach some particles. Figure 6.1(b) shows a TEM image of an ultra-thin slice of a macrophage. The nucleus $(\mathrm{N})$ and the cytoplasm can be seen, where the latter is full of vesicles (v), which have a higher electron transmission than the surrounding tissue and thus appear brighter.

$\mathrm{X}$-rays with their large penetration depth and small wavelength potentially allow a very high resolution. Especially for soft x-rays, the fabrication of diffractive lenses has strongly progressed in the last decades and current soft x-ray microscopes can achieve a spatial resolution below $15 \mathrm{~nm}$ [31]. Soft x-ray tomography was applied to image whole cells in very narrow capillaries at about $50 \mathrm{~nm}$ resolution [97]. Thus, the penetration depth is significantly increased in comparison to electron tomography, but still limited to some micrometers.

In this thesis, we use hard x-ray propagation-based phase-contrast as a lens-less near-field imaging approach. The penetration power is high enough to image samples with thicknesses in the range of some millimeters or even whole animals, as presented in the previous chapter, while theoretically the ability to resolve nanoscale structures inside the large surrounding tissue is maintained. This chapter will deal with the $2 \mathrm{D}$ and $3 \mathrm{D}$ imaging of single macrophage cells. However, as a long-range goal is in the observation of single cells inside a larger organ, we will not only focus on isolated single cells, but also try to obtain reconstructions of single cells, embedded in larger surroundings like flow chambers or resin blocks with several millimeters thickness.

\subsection{Methods}

\subsubsection{Sample preparation}

As a model system for alveolar macrophages, the mouse alveolar macrophage cell line MH-S (American Type Culture Collection, ATCC, USA) is used [107]. For cultivation, cells are maintained in RPMI medium (developed at the Roswell Park Memorial Institute) [58], supplemented with 10\% fetal calf serum (FCS), $0.05 \mathrm{mM}$ 2-mercaptoethanol and $2.06 \mathrm{mM}$ glutamine in a humidified atmosphere with $5 \%$ $\mathrm{CO}_{2}$ concentration at $37^{\circ} \mathrm{C}$. Depending on the experiment, the cells are cultivated in a Petri dish, on thin plastic membranes or in ibidi flow chambers (see Fig. 
6.3(a)), which can all be used as sample holders for the x-ray imaging experiments. In this thesis, four different preparation methods of single cells were employed in combination with different staining techniques that will be described in the following:

\section{Preparation of dried cells}

To provide a non-absorbing sample holder, thin membranes consisting of polyethylene naphthalate glued to a metal frame (MMI MembraneSlides, MMI, Germany) are used as a substrate during cell cultivation. 2 million cells suspended in $2.5 \mathrm{ml}$ medium are pipetted onto the membrane. After incubation for 4 hours, the cells are chemically fixed using $0.2 \%$ glutaraldehyde and $2 \%$ paraformaldehyde (PFA), washed two times with phosphate-buffered saline (PBS) and subsequently dried in air at room temperature. The preparation is carried out in the Max-PlanckInstitute for Experimental Medicine.

\section{Flow chambers to provide in situ conditions}

Ibidi flow chambers (see Fig. 6.3(a)) can be used as a liquid chamber for imaging experiments. They consist mainly of plastic, which has a rather high transmission for x-rays and they can be obtained with $100 \mu \mathrm{m}$ or $400 \mu \mathrm{m}$ channel height. For the flow chambers, change of the medium or the supply of chemicals is enabled via luer ports, where standard syringes can be plugged in. After incubation, cells are chemically fixed using formaldehyde and washed three-times using PBS. The flow chambers are sealed with plastic plugs and stored at $4^{\circ} \mathrm{C}$ until the experiment. A typical microscopic image of finally prepared cells in an ibidi flow-chamber can be seen in Fig. 6.3(c).

\section{Embedding cells in capillaries}

Both methods described so far are not very well suited for tomography, as they have a rather large frame that introduces a large missing wedge. To enable artifactfree 3D imaging, we tried to prepare cells inside capillaries. To this end, cells are cultivated as usual in any kind of container. After incubation, the cells are washed and centrifuged to obtain a cell pellet. The pellet is resuspended with $1 \%$ lowmelting agarose (NuSieve GTG agarose, FMC Bioproducts) in 4\% PFA in PBS that was kept in a water bath at $42^{\circ} \mathrm{C}$ to keep it liquid. A cut glass capillary (Hilgenberg, Germany) with $500 \mu \mathrm{m}$ diameter and $10 \mu \mathrm{m}$ wall thickness is then 
held into the cell suspension. By capillary forces the suspension is sucked into the capillary, which is subsequently sealed with wax on both sides. The closed capillaries are glued to a sample holder and stored at $4^{\circ} \mathrm{C}$ until the experiment.

\section{Embedding cells in resin}

As an alternative to capillaries, the cells can be embedded in resin as it is usually done for electron microscopy. This has the advantage that the sample is solid and can be tailored to different forms. For resin embedment, cells are cultivated in plastic Petri-dishes. After incubation, the cells are dehydrated by an ascending series of ethanol and washed three times with $100 \%$ ethanol, each for five minutes. A liquid resin called Epon (Serva, Germany) is prepared. It consists of glycidether 100, DDSA, MNA and DMP-30. The ethanol in the cells is then slowly substituted by epon in an ascending series, ending with $100 \%$ epon. Incubation times are 30 minutes each. The last step with $100 \%$ epon takes one hour and the liquid resin is held by little plastic tubes (BEEM capsules, Agar scientific, United Kingdom) to avoid melting. After the full substitution, the sample is baked for 24 hours at $60^{\circ} \mathrm{C}$. The Petri-dish with cells is cut out to obtain blocks with the capsules by milling away unnecessary resin (see Fig. 6.2(a)). Finally, the samples are cut into little disk-shaped pieces that are subsequently polished and cut with a

(a)

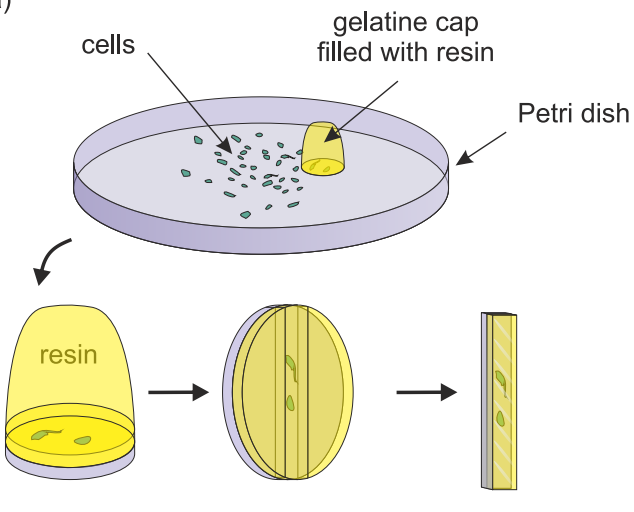

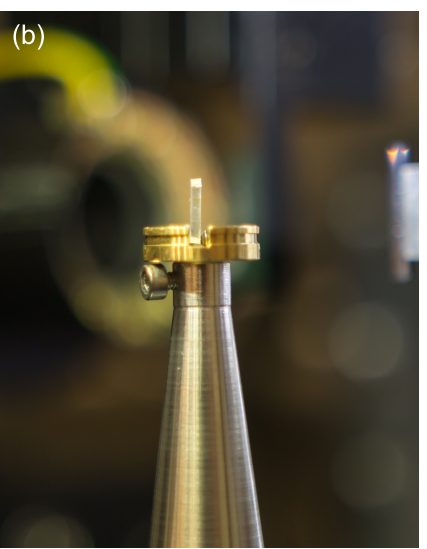

Figure 6.2: (a) sketch of the preparation procedure for resin embedded cells. Cells are embedded in resin and a small stick is cut out that is mounted in a sample holder. (b) shows a photograph of a finally prepared epon/plastic stick mounted in a sample holder. 
precision wafer-saw to reduce surface roughnesses in order to obtain little sticks of about $2 \mathrm{~mm}$ diameter as sketched in Fig. 6.2(a). During the last steps, it is important to keep the boundary surface of the Petri-dish, as cells are located at the intersection between plastic and epon. Figure 6.2(b) shows a photograph of a finished epon/plastic stick, mounted to a sample holder in the GINIX setup.

\subsubsection{Staining procedures}

As typical diameters of single macrophage cells are in the range of about 10 to $20 \mu \mathrm{m}$ and interaction constants of the cell materials are rather weak, the contrast of unstained cells may be very low. In particular if cells are embedded in surrounding material (water, resin or the lung), the contrast formation is based on the relative change of the refractive index

$$
\Delta n=n_{\text {cell }}-n_{\text {surrounding }},
$$

which may be even less. For a typical biological material $\left(\mathrm{H}_{50} \mathrm{C}_{30} \mathrm{~N}_{9} \mathrm{O}_{10} \mathrm{~S}_{1}, \rho=\right.$ $1.35 \mathrm{~g} / \mathrm{cm}^{3}$ ) embedded in water, the real part of the difference is $\Delta n \simeq 3.6 \cdot 10^{-7}$ compared to a difference to air of $n_{\text {cell }}-1 \simeq 1.6 \cdot 10^{-6}[71]$. Thus, it can be expected that also the contrast will be 50 times lower. One way to increase it again is to stain the cells with heavy metals, which have a stronger interaction with the x-rays. In this work, several staining procedures were tested that are briefly described in this section.

\section{Barium stain}

A contrast agent that is routinely used in medical imaging is barium sulfate $\left(\mathrm{BaSO}_{4}\right)$. Typically, patients have to drink the milk-like suspension of bariumsulfate particles if their digestive system is examined. It is very insoluble in water and thus poses no threat to human health. For staining of MH-S cells, 6 million cells in $3 \mathrm{ml}$ medium are coincubated with 1 to $10 \mu \mathrm{l}$ of commercially available barium sulfate (Micropaque CT , Guerbet, Belgium) for 4 hours. The ready-to-use contrast agent contains $7.5 \mathrm{~g} \mathrm{BaSO}_{4}$ per $150 \mathrm{ml}$. Figure 6.3(d) shows a micrograph of cells with a 20-fold magnification (Axio Observer, Zeiss, Germany) after incubation with $1 \mu \mathrm{l}$ contrast agent in comparison to unstained cells, shown in Fig. $6.3(\mathrm{c})$. 
(a)
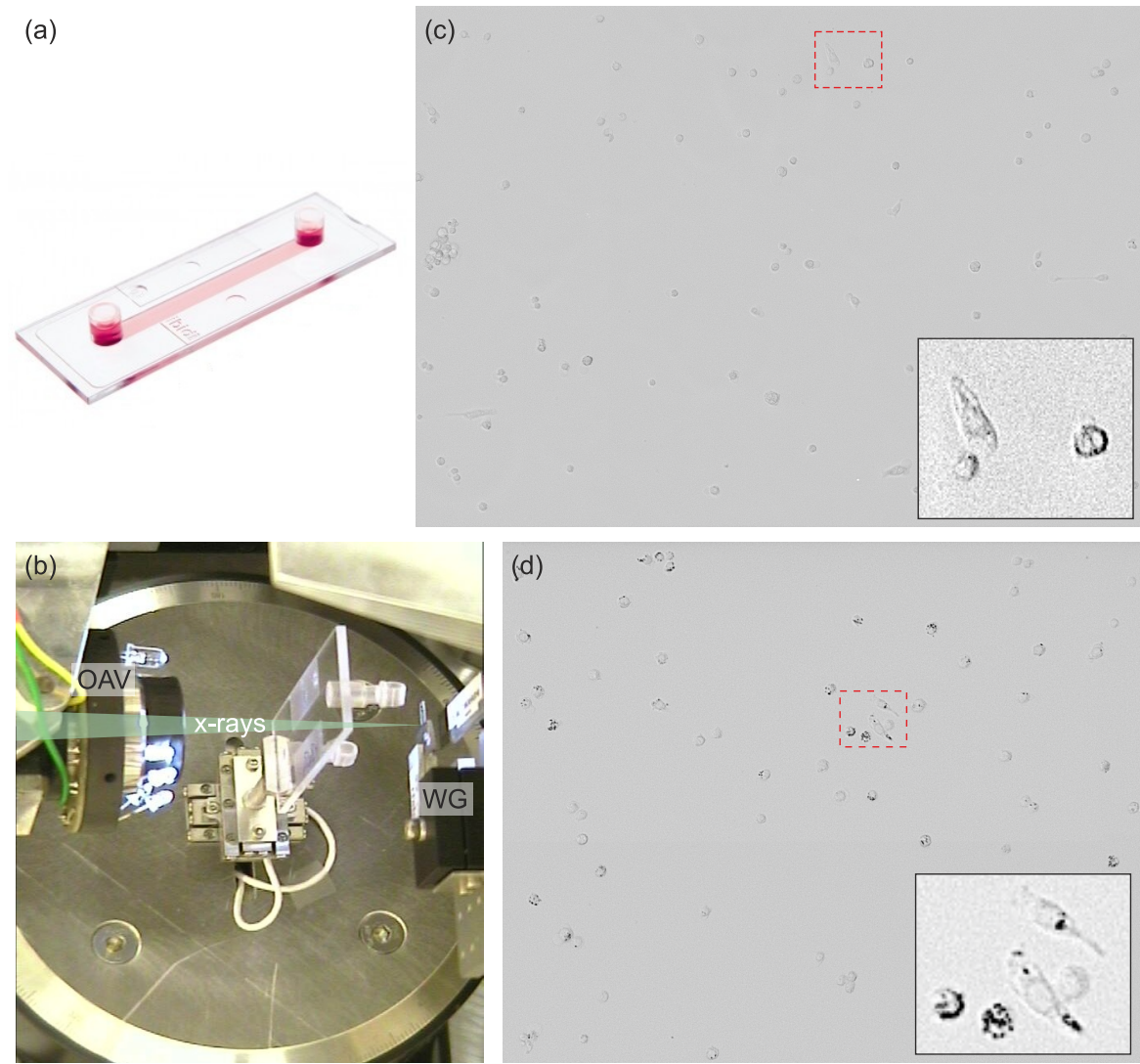

Figure 6.3: (a) ibidi flow chamber with a red colored liquid inside the channel, where cells are located. (b) photograph of an ibidi chamber mounted in the experimental setup at the GINIX setup, showing also a waveguide (WG) and the on-axis-view microscope $(O A V)$. (c) micrograph of cells in an ibidi flow-chamber without contrast agents. (d) micrograph of cells in an ibidi chamber, which were loaded with barium-sulfate particles. The insets in (c) and (d) show the regions marked by the dashed rectangle magnified and with adjusted contrast.

\section{Osmium stain}

The conventional staining procedure for electron microscopy involves staining lipids with osmium tetroxide $\left(\mathrm{OsO}_{4}\right)$. As $\mathrm{OsO}_{4}$ binds to lipids, it is highly toxic and only used for preparations where the final sample is fixed in a resin block. To this end, the cells are stained with $2 \% \mathrm{OsO}_{4}$ in PBS for 4 hours before the ascending dehydration series, while all other preparation steps remain unchanged. 


\section{Antibody stain with gold enhancement}

To obtain a specific staining of structures of interest inside a cell, antibodies that bind to specific receptors are used in fluorescence imaging with visible light. To obtain a significant contrast in x-ray imaging, secondary antibodies conjugated to nanogold particles are used and a subsequent gold enhancement is performed. To this end, cells are cultivated in an ibidi chamber or on a membrane slide, fixed with formaldehyde and washed with PBS. Subsequently, the cells are permeabilized using $0.2 \%$ Triton X-100 and $1 \%$ normal goat serum (NS) in PBS for 5 minutes on ice, followed by washing with $1 \%$ NS in PBS 3 times. The cells together with the primary antibody (rabbit anti-CD68) are incubated for $1 \mathrm{~h}$ at room temperature using a gyratory rocker, followed by another washing procedure with 1\% NS and $5 \%$ bovine serum albumin (BSA) in PBS. $10 \mu \mathrm{g} / \mathrm{ml}$ of the secondary antibodies (Alexa Fluor 594 fluoronanogold) are diluted in PBS with 1\% BSA and rotated on the rocker for one hour in the dark, followed by washing with pure PBS. For the gold-enhancement the cells are washed with tris-buffered saline (TBS) and distilled water followed by a special gold-enhancement kit (Gold Enhance EM Plus, Biotrends). Finally the cells are washed again with distilled water.

\subsubsection{Experimental parameters}

The cell imaging experiments are performed at the GINIX instrument at DESY and at the ID16a beamline of the ESRF. Table 6.1 gives an overview of the different experimental parameters used in the $2 \mathrm{D}$ imaging experiments, all carried out with a waveguide illumination at the GINIX instrument. Table 6.2 shows the most important experimental parameters for tomographic experiments carried out with a waveguide illumination as for the $2 \mathrm{D}$ case and in a $\mathrm{KB}$ setting for one experiment at the ID16a beamline. Samples for the experiments are prepared as described in the previous sections. In the table the different preparation types are denoted as: (i) dried - for the cells prepared on membrane slides that are chemically fixed and dried, (ii) epon - for the resin embedded cells, (iii) ibidi - for cells prepared in the ibidi flow-chambers under in situ conditions and (iv) capillary - for the cells prepared with agarose gel in a thin glass capillary. The dried and ibidi samples are only used for 2D imaging experiments, as the missing wedge in a rotational scan would be too large. The epon samples and the cells embedded in a capillary are also used for phase-contrast tomography measurements, in which the internal structure of the cells and the position of contrast agents are of interest.

All experiments in this chapter share the same principle geometry that is sketched 


\begin{tabular}{lllll}
\hline & Fig. 6.5 & Fig. 6.6 & Fig. 6.7(a,b) & Fig. 6.7(c,d) \\
\hline sample & dried & dried & epon & epon \\
eff. pixel size $[\mathrm{nm}]$ & 29.3 & 22.9 & $2 \times 38.5$ & $2 \times 37.8$ \\
min. $x_{01}[\mathrm{~mm}]$ & 23.0 & 18.0 & 30.2 & 29.6 \\
$x_{02}[\mathrm{~m}]$ & 5.13 & 5.13 & 5.12 & 5.12 \\
energy $[\mathrm{keV}]$ & 13.6 & 13.6 & 8.0 & 8.0 \\
$F^{10}$ & 0.042 & 0.033 & 0.032 & 0.031 \\
waveguide & $59 \mathrm{~nm}$ & $59 \mathrm{~nm}$ & taper & taper \\
number of distances & 1 & 4 & 1 & 2 \\
exposure time $[\mathrm{s}]$ & 10 & 10 & 0.2 & 0.1 \\
\hline
\end{tabular}

\begin{tabular}{lllll}
\hline & Fig. 6.7(e,f) & Fig. 6.8 & Fig. 6.9 & Fig. 6.10 \\
\hline sample & epon & ibidi & ibidi & ibidi \\
eff. pixel size $[\mathrm{mm}]$ & $2 \times 24.6$ & $2 \times 25.0$ & 31.8 & 51.0 \\
min. $x_{01}[\mathrm{~mm}]$ & 19.3 & 19.6 & 24.7 & 39.6 \\
$x_{02}[\mathrm{~m}]$ & 5.12 & 5.12 & 5.07 & 5.07 \\
energy $[\mathrm{keV}]$ & 8.0 & 8.0 & 13.8 & 13.8 \\
$F^{10}$ & 0.020 & 0.021 & 0.046 & 0.074 \\
waveguide & taper & taper & $59 \mathrm{~nm}$ & $59 \mathrm{~nm}$ \\
number of distances & 4 & 5 & 8 & $3 / 5^{*}$ \\
exposure time $[\mathrm{s}]$ & 0.2 & 2 & 10 & $10 / 1^{*}$ \\
\hline
\end{tabular}

Table 6.1: Overview of experimental parameters used for 2D imaging. Exposure times are stated per image. The waveguides were a crossed multilayer waveguide with $59 \mathrm{~nm}$ guiding core $(59 \mathrm{~nm})$ and a bonded air/silicon waveguide in tapered geometry (taper). For some images the effective pixel size is resampled by a factor of 2 to increase the SNR.*: The first number is valid for Fig. 6.10(a,b) and the second for $(c, d)$.

in Fig. 4.6 and 4.11. The x-rays are generated in an undulator and focused to a small spot, which acts as an effective secondary source. For the experiments at the ID16a beamline, the size of the focus (designed to reach 10 to $20 \mathrm{~nm}$ ) determines the maximum possible resolution. A lens-coupled detector equipped with a Frelon camera is used for the imaging, resulting in a detector pixel size of $p=0.84 \mu \mathrm{m}$. The total photon flux is in the order of $10^{12}$ photons per second. For the waveguide experiments the KB-focus is larger. However, due to the used waveguides a much smaller effective source size in the range of 13 to $23 \mathrm{~nm}$ is achieved [14]. Here, a fiber-coupled detector with a pixel size of $p=6.54$ is used. To still fill the whole FOV, the fiber-coupled detector is generally placed at a larger distance $x_{02}$, which also leads to smaller Fresnel numbers $F^{10}$. 


\begin{tabular}{lllll}
\hline & Fig. 6.13/6.14 & Fig. 6.15 to 6.18 & Fig. 6.19 & Fig. 6.20(a,b) \\
\hline sample & capillary & epon & epon & epon \\
eff. voxel size $[\mu \mathrm{m}]$ & $2 \times 25.4$ & 50.7 & $2 \times 38.5$ & $2 \times 24.6$ \\
min. $x_{01}[\mathrm{~mm}]$ & 19.7 & 20.2 & 30.2 & 19.3 \\
$x_{02}[\mathrm{~mm}]$ & 5.07 & 0.34 & 5.12 & 5.12 \\
energy $[\mathrm{keV}]$ & 13.8 & 17.0 & 8.0 & 8.0 \\
$F^{10}$ & 0.037 & 0.19 & 0.032 & 0.020 \\
waveguide & $59 \mathrm{~nm}$ & - & taper & taper \\
number of distances & 1 & 4 & 1 & 4 \\
number of projections & 360 & 1000 & 1020 & 2040 \\
angular range $\left[{ }^{\circ}\right]$ & 360 & 180 & 180 & 360 \\
exposure time $[\mathrm{s}]$ & 1 & 0.25 & 0.2 & 0.2 \\
\hline
\end{tabular}

Table 6.2: Overview of experimental parameters used for $3 D$ imaging. Waveguides are denoted as in Tab. 6.1 and exposure times are stated per image.

Two different waveguides are used: A crossed multilayer waveguide with $59 \mathrm{~nm}$ guiding core yielding up to $2 \cdot 10^{8}$ photons per second, denoted as $59 \mathrm{~nm}$ in Tab. 6.1 and 6.2 , and a bonded silicon waveguide with an empty, air-filled guiding channel in a tapered geometry that delivers up to $10^{10}$ photons per second, denoted as taper. While the crossed multilayer waveguide is well suited for higher energies, the bonded version has the advantage of an up to 50 times higher flux density even at lower energies. However, the far-field of the tapered waveguide showed a much lower stability in the experiments. Due to the taper angle, the position of the incident KB-beam has a larger influence than in a straight channel and slightly different illuminations of the waveguide may change the resulting far-field significantly.

\subsubsection{Waveguide-stripe removal}

In the crossed multilayer waveguide, the reduction of the spot size in the focus does not occur simultaneously for both directions perpendicular to the optical axis. Rather, the beam is first confined in one direction, while in the other direction the confinement occurs about $385 \mu \mathrm{m}$ farther, where the first $1 \mathrm{D}$ waveguide has its exit plane. This leads to effectively astigmatistic source, which causes slightly different magnifications and therefore different Fresnel numbers for the horizontal and vertical direction. Furthermore, regular stripe artifacts can be observed in the empty-beam images that may disturb the empty-beam correction and subsequent phase-retrieval algorithms if the waveguide is not perfectly stable. 

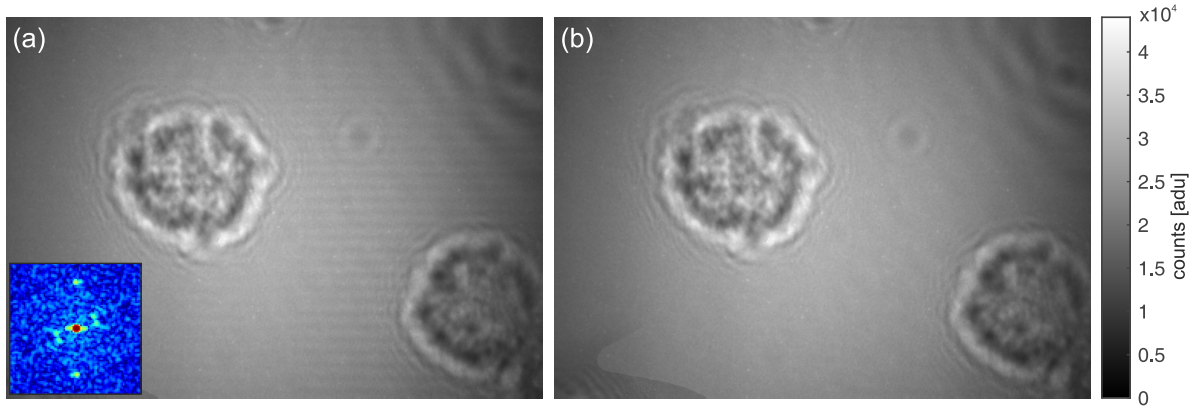

Figure 6.4: Removal of stripe artifacts that are caused by the crossed multilayer waveguide. (a) uncorrected raw image that shows two distinct peaks in vertical direction in the PSD, whose central part is shown as an inset. (b) corrected raw image, where the peaks have been replaced by average values with a similar radius to the central peak in the $P S D$. The structures at the edges stem from the flat-field of the detector that changed over time and was not calibrated correctly.

Figure 6.4(a) shows a typical raw image without empty-beam correction obtained with a crossed multilayer waveguide. The inset at the lower left corner shows the central part of the corresponding power spectral density (PSD). Next to the strong central peak, two symmetric peaks on the vertical axis can be observed, which correspond to a regular horizontal pattern in the real space image. Before further processing of the images, a mask is defined that marks these two peaks in the PSD. The images are then altered such that the mask is rotated by $10^{\circ}$ and the mean PSD values after rotation are used to replace masked pixels in Fourier space. After Fourier back-transform, the regular stripe pattern disturbing the image impression is fully removed and no significant artifacts due to this correction are introduced, as can be seen in in Fig. 6.4(b). The artifacts in the upper right and lower left corner are caused by a wrong flat-field of the camera. However, these artifacts are fully corrected by the empty-beam division, as they do not experience any free-space propagation.

\subsection{D Imaging of single macrophages}

\subsubsection{Dried macrophage cells}

Figure 6.5(a) shows an uncorrected phase-contrast image of a single, dried macrophage that was coincubated with barium sulfate during the preparation. The raw 
image is corrected by an empty-beam image. Additionally, outliers and a residual low-frequency variation are removed. For the latter, averaged profiles are calculated at the edges of the images that are used to linearly interpolate a correction image, by which the raw hologram is divided. The result is shown in Fig. 6.5(b), with a normalized uniform background. If the Fresnel number is regarded in the effective geometry

$$
F=\frac{p_{e f f}^{2}}{\lambda x_{e f f}}=\frac{p / M^{2}}{\lambda z_{12} / M}=\frac{p}{\lambda z_{12} M},
$$

it can be seen that a larger magnification $M$, leads to a smaller Fresnel number, if the change in $x_{12}=x_{02}-x_{01}$ is negligible, which is always true in the present experiments as $x_{02} \gg x_{01}$. Thus, if small structures are of interest and a large magnification is chosen, the Fresnel number is very small. Hence, images are in the holographic regime and fine structures cannot be observed without phase retrieval due to the presence of many interference fringes. In exchange, the contrast of the almost non-absorbing sample is strongly increased and even very small barium clusters located outside the cell can be clearly identified.

\subsubsection{Single-distance phase-retrieval}

To extract real-space information from the measured holograms, phase-retrieval algorithms have to be used. Three different single-distance approaches are compared using the normalized hologram shown in Fig. 6.5(b). For the first approach we use CTF-based phase-retrieval with the assumption of a single material object (Eq. (2.33), $m=1$ ), i.e., a constant ratio $\kappa=\delta / \beta$ is present over the whole image. The coupling ratio is chosen to $\kappa=150$ based on visual inspection and high frequencies are regularized by choosing $\alpha_{2}=0.05$ (see section 2.2.2). The resulting phase map is depicted in Fig. 6.5(c). As a second approach, a modified HIO algorithm (Eq. (2.52)) is employed, where a pure phase object, a compact support of the object and positivity of $\delta$ are assumed for the sample-plane projection $P_{s}$. The support for the HIO reconstruction is automatically determined by thresholding the phase map obtained with the CTF reconstruction, followed by image dilatation and erosion to create a closed mask. Figure 6.5(d) shows the resulting phase-map after 258 iterations, where soft projections (Eq. (2.54)) with $D=0.027$ are used and the algorithm is stopped using the discrepancy principle. The last approach is a simple holographic reconstruction as described in Eq. (2.24), where the measured intensities are back-propagated to the object plane. The resulting phase-distribution is shown in Fig. 6.5(e). To enable a better comparison of the different reconstructions, the azimuthally averaged power spectral densities for all 

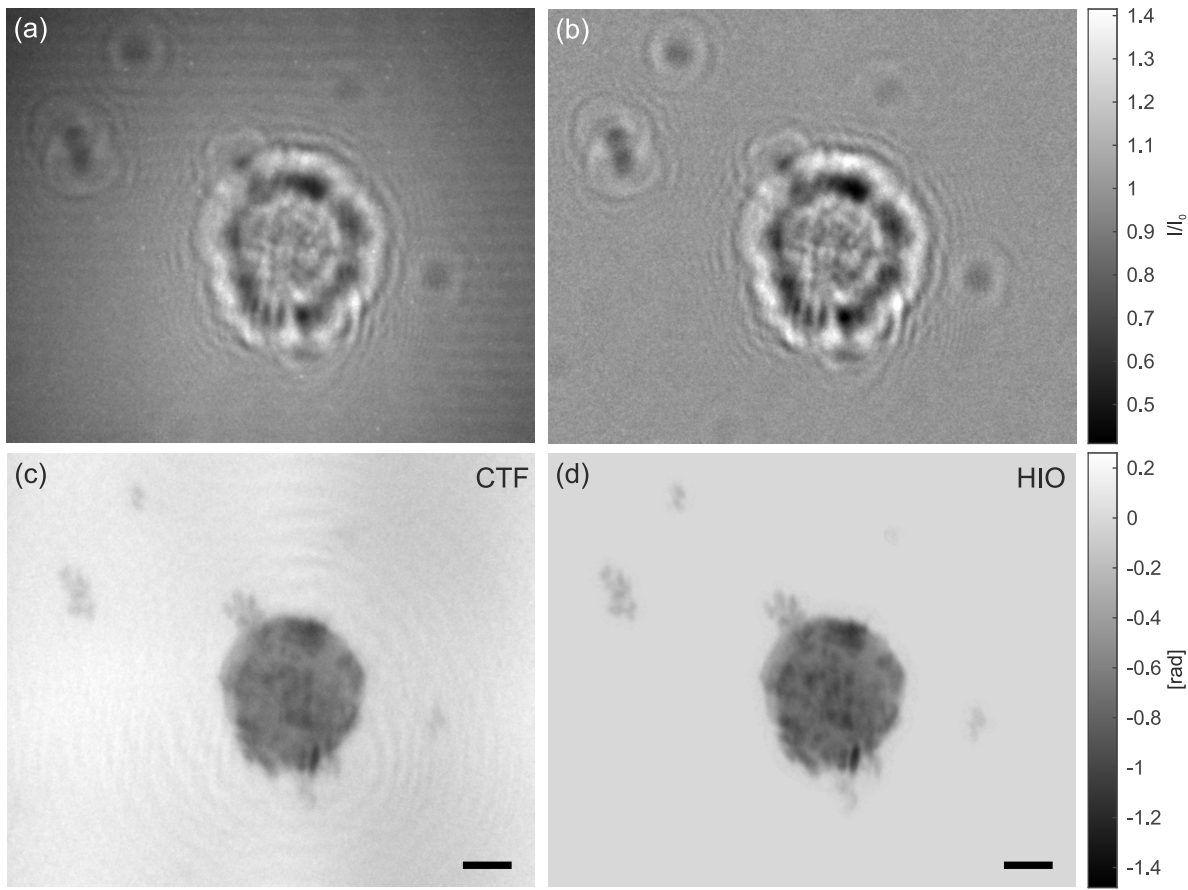

(e)
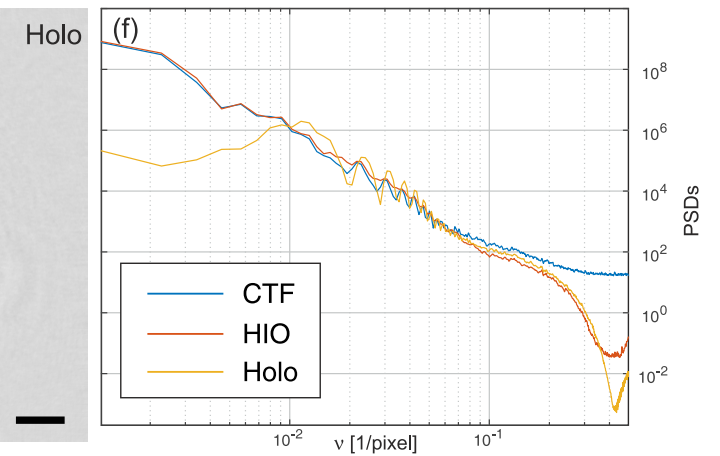

Figure 6.5: Single-distance phase-retrieval of a dried, barium-sulfate stained macrophage cell. (a) shows the raw image recorded with the Pandora detector. (b) shows the empty-beam corrected hologram where waveguide stripes, residual profiles and outliers are removed. (c) shows the phase map, retrieved with the CTF-based approach. (d) shows the phase map after iterative reconstruction with a modified HIO algorithm and (e) shows the holographically reconstructed phase. (f) shows the PSDs of the three reconstructions on a double logarithmic scale. All scale bars denote $5 \mu \mathrm{m}$. 
reconstructions are shown in Fig. 6.5(f).

The holographic reconstruction (Fig. 6.5 (e)), in which no additional constraints are used for the phase retrieval, shows fine structures of the image and single barium particles in the clusters outside the cell can be distinguished. No regularizing parameters have to be chosen and as the reconstruction takes less than one second on a personal computer, this method is perfectly suited as a real-time feedback during the measurements. However, the reconstructed image is disturbed by the twin-image as expected from the theory. This strongly affects the low frequencies, so that reliable quantitative values about the projected density cannot be obtained. The twin image manifests itself also in the PSD, where oscillations can be observed, corresponding to the zeros in the PCTF.

The CTF-based phase-retrieval (Fig. 6.5 (c)) as a deterministic approach also has the advantage that calculations for the reconstruction are very fast (typically below one second). Thus, the determination of necessary regularization parameters can be easily performed. This reconstruction corresponds much better to an expected density map of a single cell and the twin-image influence is suppressed quite well. The cell body of the macrophage can be observed next to several barium particles that are located in the cell's cytoplasm. However, as just a single distance was used, the influence of zeros in the PCTF can be still observed and weak fringes around the cell can be identified.

The best reconstruction is obtained with the HIO algorithm (Fig. 6.5 (d)), for which also the most a priori assumptions to the object are posed. The 258 singledistance HIO-iterations take about 3.5 minutes on the same computer as used before, which makes the right choice of reconstruction parameters a time-consuming process. The reconstruction can be accelerated to about 30 seconds by using a state-of-the-art GPU (Geforce GTX Titan, 6 GB memory), which is not always available. Note that in particular the low frequencies correspond very well to those of the CTF reconstruction, although the HIO algorithm was initialized with a random phase distribution with values between $\phi_{0} \in[-1.2 \ldots 0] \mathrm{rad}$. At the high spatial frequencies, the PSD of the HIO reconstruction decreases to smaller values, indicating a lower noise-level of the reconstruction, which is in good agreement with the visual impression of the image. The cross-over to the noise level transpires at around 0.3 periods/pixel, corresponding to a half-period resolution of $49 \mathrm{~nm}$. 
(a)

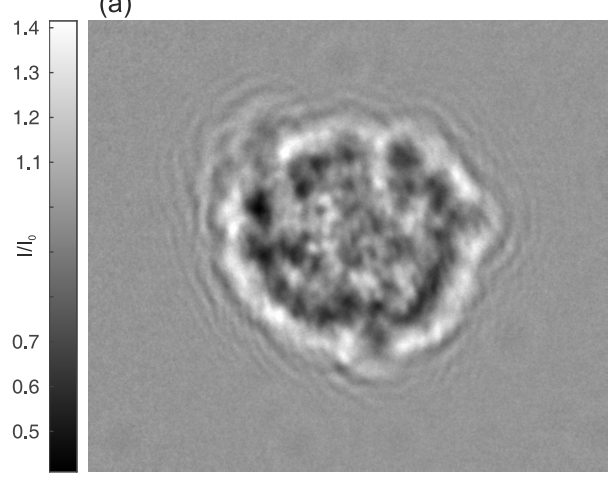

(c)

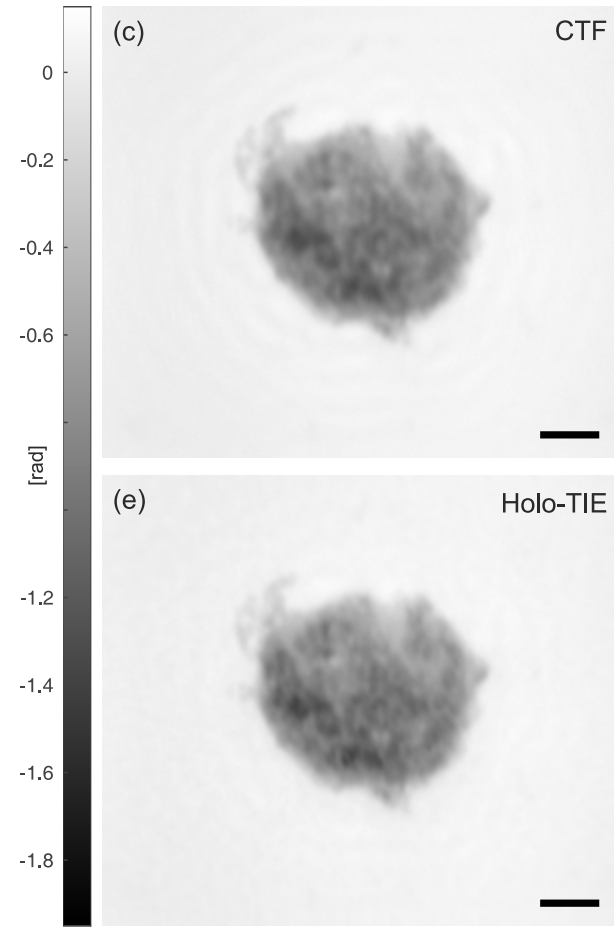

(b)

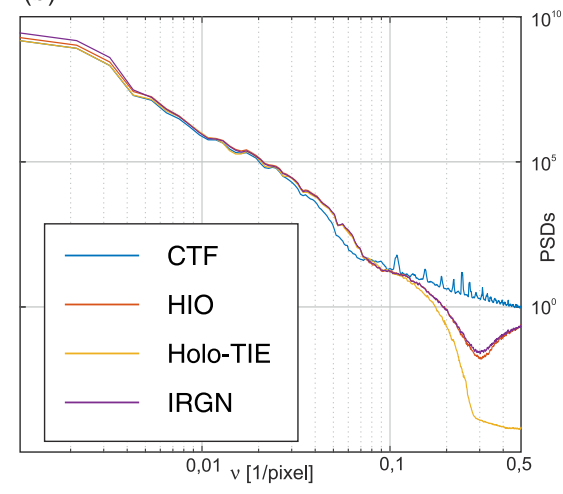

(d)

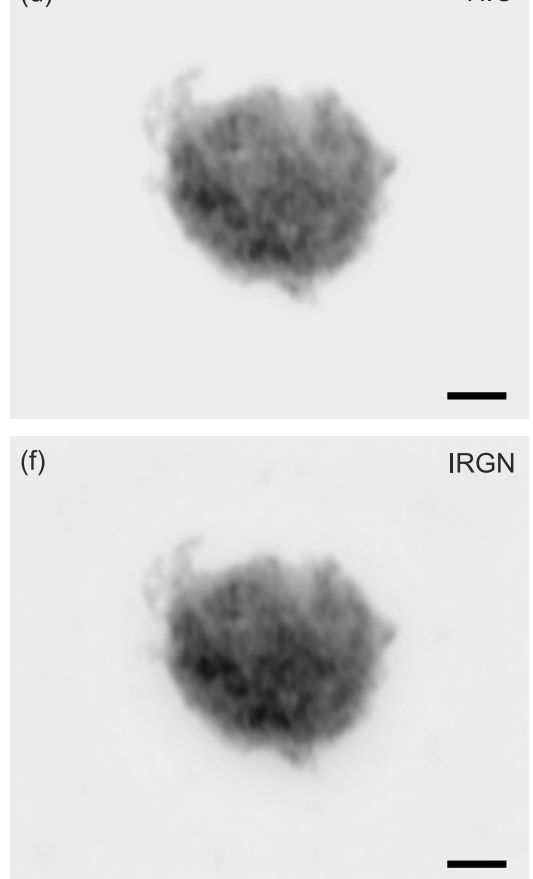

Figure 6.6: Multi-distance phase-retrieval using measurements at four different Fresnel numbers of a dried, barium-sulfate stained macrophage cell. (a) shows a hologram with all corrections applied as before. (b) shows the PSDs of the four reconstructions shown in (c) to (f), where (c) shows the phase map obtained with a CTF-based phase-retrieval, (d) shows the iterative modified HIO reconstruction using an automatically determined support, (e) shows the phase obtained using a combined Holo-TIE reconstruction (see main text) and (f) shows the result obtained with the IRGN approach. Information about a compact support was only applied for the HIO reconstruction. All scale bars denote $5 \mu \mathrm{m}$. 


\subsubsection{Multi-distance phase-retrieval}

Figure 6.6 shows the application of multi-distance phase-retrieval to an isolated, dried macrophage loaded with barium sulfate. The measured, raw intensitydistributions are corrected as for the single-distance case and one of the four resulting holograms is shown in Fig. 6.6(a). Images at different Fresnel numbers are recorded by changing the source-to-object distance $x_{01}$, while keeping the source-detector distance $x_{02}$ fixed. To enable phase retrieval with multiple distances, all projections have to be precisely aligned and rescaled to each other. To this end, the exact knowledge of the distances $x_{01}$ and $x_{02}$ is necessary, to calculate correct magnification factors. Images recorded with a lower magnification are magnified using bicubic interpolation, such that all images have the smallest pixel size. Subsequently, images are aligned to each other with an FFT-based correlation algorithm for fast sub-pixel image registration [62]. For the case shown in Fig. 6.6(a) no further treatment of the data is necessary to obtain the correct shift values. However, in many cases background variations cannot be well corrected and/or noise deteriorates the alignment. In these cases, the images used for the registration are low- and high-pass filtered prior to the registration. Additionally, in cases where the Fresnel numbers differ strongly, a single-distance CTF-based phase-retrieval can be applied first, to allow the registration of the coarsely reconstructed phase-maps. If the right shift values are determined, images are shifted with sub-pixel accuracy by multiplication with a phase factor in Fourier space. For the reconstruction in Fig. 6.6, distances between $x_{01, z} \in[18.0,19.5$, , 21.5, 22.5] $\mathrm{mm}$ are used, where for the Holo-TIE reconstruction the middle two distances are used. The astigmatism of the waveguide is accounted for by using a different defocus distance for the $y$-direction: $x_{01, y}=x_{01, z}+0.385 \mathrm{~mm}$.

In Fig. 6.6(c) to (f), the result of four different phase-retrieval approaches using the CTF-based approach (c), the modified HIO (d), a combined Holo-TIE approach (e) and the iterative newton approach (f) are shown. All reconstructions deliver similar results, with slight differences that can be best quantified by analyzing the PSDs, depicted in Fig. 6.6(b). In general, the cell in Fig. 6.6 has taken up more barium than that in Fig. 6.5, which is reflected in the larger modulus of mean phase-shift values. For the CTF reconstruction, $\kappa=50$ and $\alpha_{2}=10^{-5}$ have been chosen. The HIO runs with soft projections $(D=0.02)$ and is stopped after 500 iterations, as the error stagnates after a certain number of iterations. The 500 multi-distance iterations take about 300 seconds with a GPU implementation.

To compensate a strong influence of noise in the Holo-TIE approach, a low- 
frequency image is used to create a combined detector-plane phase as described by Eq. (2.46). However, instead of using a measurement with a larger Fresnel-number difference $\Delta F^{10}$ (see section 2.2.3.2), we used the phase-map $\phi_{\mathrm{CTF}}$, obtained with the CTF approach, to calculate a low-frequency image by

$$
\phi_{2}^{\prime}=\arg \left(\mathcal{D}_{x}\left(\mathrm{e}^{\mathrm{i} \phi_{\mathrm{CTF}}}\right)\right)
$$

where a pure phase object was assumed. This detector-plane phase-distribution is used to calculate the combined phase $\phi_{\text {comb. }}^{\prime}$ by employing Eq. (2.46) with a threshold of $\nu_{\text {cut }}=0.01$ periods $/$ pixel and a high-frequency phase-map $\phi_{1}^{\prime}$ obtained by solving the Holo-TIE equation (2.40). The distances used for the Holo-TIE reconstruction differ by $\Delta x=2 \mathrm{~mm}$, leading to a Fresnel number difference of $\Delta F^{10}=3.6 \cdot 10^{-3}$.

For the iterative Newton approach, a pure-phase assumption and positivity of $\delta$ is enforced, but no support information about the object is used. The algorithm is stopped after a residual reduction $\Delta d \leq 0.01$ is reached (see Eq. (2.53)), which leads to a total reconstruction time of about 500 seconds with a GPU implementation. The reconstruction times for the Holo-TIE and CTF approach are still below 1 second.

In general, the use of multiple distances will improve the phase retrieval. Upon inspection of the PSDs (Fig. 6.6(b)) it becomes clear that even the CTF based reconstruction does not show significant deviations due to zeros in the PCTF. Compared to the HIO and IRGN reconstructions, slight deviations at very low frequencies can be observed, leading to some differences in the absolute phasevalues. While the HIO and IRGN perform very similar at high spatial frequencies, the CTF reconstruction does not reach very small values. In contrast, the combined Holo-TIE reconstruction shows the strongest decrease to small values at high frequencies. As this was an indicator for a better noise performance, the Holo-TIE approach seems to deliver the best results for the case shown here. The PSD shows a cross-over to the noise level at about 0.25 periods/pixel, corresponding to $46 \mathrm{~nm}$ half-period resolution.

A major point to ensure high quality reconstructions is the alignment of the images. In particular for tomographic 3D imaging, the alignment of projections at different distances may pose challenges, especially if the sample changes over time. The CTF approach is most robust, as it does not rely on any consistency of the data. Even for misaligned projections, a blurred phase-map will be calculated by the CTF reconstruction, where other algorithms would diverge. Especially residual 
low-frequency variations in the image will cause the failure of many reconstructions. As in most cases the artifacts introduced by the CTF phase-retrieval will not be the limiting factor for a high quality $3 \mathrm{D}$ reconstruction, we will mainly use the CTF-based phase-retrieval for holographic tomography experiments.

\subsubsection{Stained and unstained cells embedded in resin}

So far we have investigated the influence of phase retrieval on isolated dried macrophages on transparent substrates that were loaded with barium sulfate. However, although the cells are chemically fixed, their structure will deteriorate during the drying process, so biologically relevant information is limited. A preparation method that better preserves the ultrastructure of cells is the controlled dehydration followed by embedding the cells in resin. In combination with osmium tetroxide staining, this method is widely used for electron microscopy. Unstained macrophages and cells loaded with barium sulfate are prepared in this way, both with and without osmium tetroxide staining to enhance the contrast of lipids.

Figure 6.7 shows empty-beam corrected, holographic projections obtained from different plastic/epon samples that were prepared as sketched in Fig. 6.2 and their corresponding phase-maps, retrieved using the CTF reconstruction. The hologram and the phase map of the unstained (blank) cells is shown in in Fig. 6.7(a) and (b). Although hardly any contrast is present in the holograms, two single cells can be recognized in the phase map, which are marked by arrows. Note that although more than a single distance were recorded, only the first distance is used as motion between the different imaging planes could be observed. For osmium stained cells, shown in Fig. 6.7(c) and (d), the cells can be clearly identified in the holograms as well as in the reconstructed phase distribution. The cells labeled with barium and osmium are shown in Fig. 6.7(e) and (f).

To quantify the increase in contrast, regions inside the cell and regions next to it are marked and the mean phase difference is calculated. While for the blank cells, a relative phase difference to the surrounding regions of pure resin is in the range of about

$$
\Delta \phi_{\text {blank }}=(0.13 \pm 0.06) \mathrm{rad},
$$

the osmium stain increases this difference by about a factor of 3 to

$$
\Delta \phi_{\mathrm{OsO}_{4}}=(0.38 \pm 0.03) \mathrm{rad} .
$$

For osmium stained cells that are additionally loaded with barium sulfate, the 

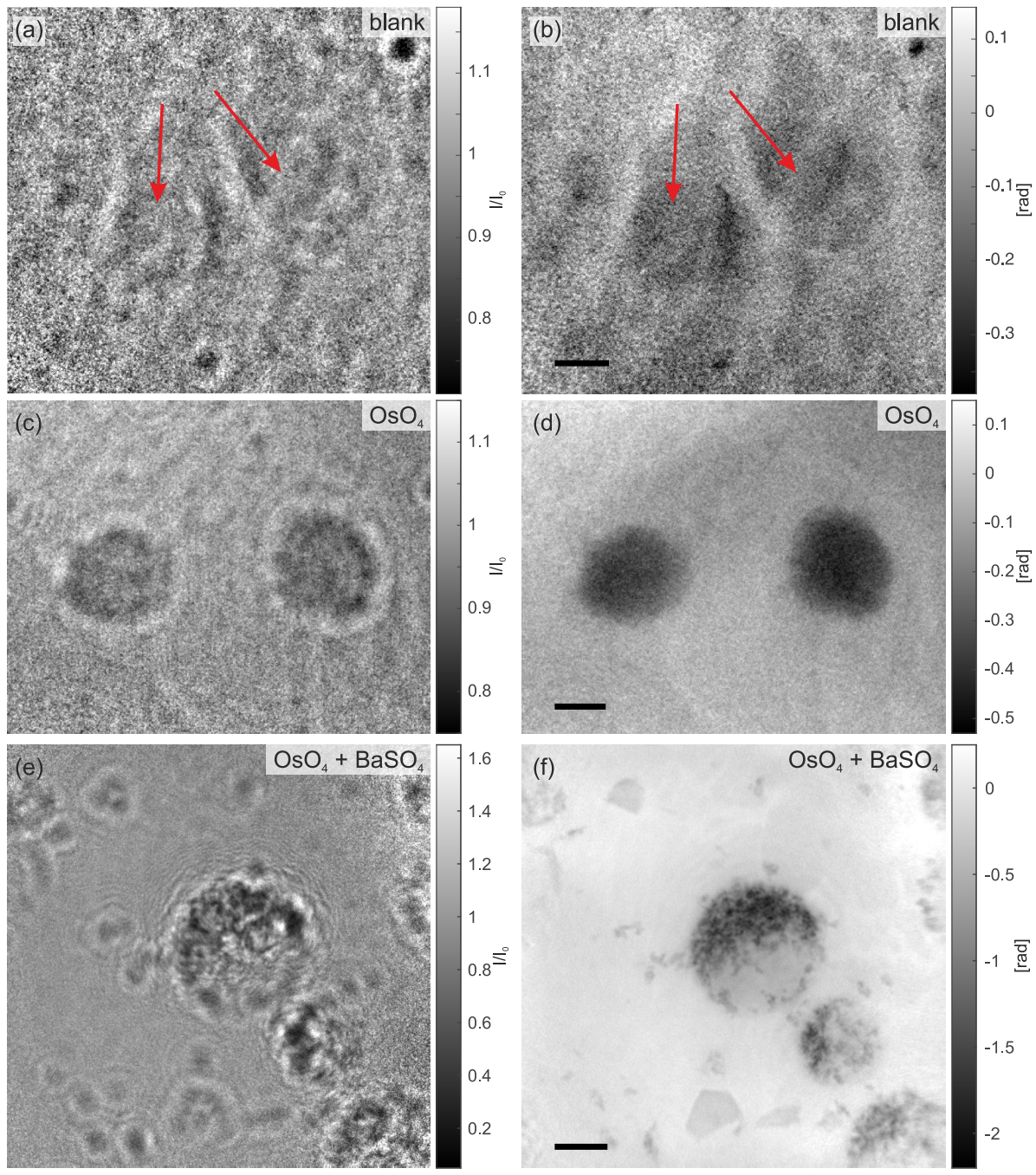

Figure 6.7: Comparison of empty-beam corrected holograms (left) and resulting CTF phase-maps (right) of cells labeled with different contrast agents: (a) hologram of blank cells without contrast agents and the (b) CTF retrieved phase-map using a single distance. The arrows mark the position of two cells. (c) Hologram of osmium tetroxide stained cells, showing a significantly increased contrast. (d) Reconstructed phase distribution from (c). (e) Hologram of cells labeled with osmium tetroxide and barium sulfate showing the strongest increase in contrast. (f) phase map obtained with CTF phase-retrieval using 4 distances. Single barium precipitates can be observed additionally to the cell surrounding. All scale bars denote $5 \mu \mathrm{m}$. 
strongest increase in contrast can be observed, with a maximum phase difference of

$$
\Delta \phi_{\mathrm{OsO}_{4} \& \mathrm{BaSO}_{4}}=(1.95 \pm 0.04) \mathrm{rad} .
$$

To ensure comparability of the values, all phase-maps are reconstructed using the same ratio of $\kappa=15$. The high frequencies are regularized with $\alpha_{2}=0.1$ for (a) to (d) and with $\alpha_{2}=0.01$ for (e) and (f), as for the latter four distances are used to compensate the influence of zeros in the PCTF. For the reconstructions in (c) and (d) only two distances were used, as the sample seemed to be tilted at the third distance. For (a) and (b), only a single distance was successfully measured as the waveguide was misaligned during the scan.

For the cells stained with barium sulfate and osmium tetroxide (Fig. 6.7(e) and (f)), the cell body as well as many barium particles can be observed at the same time. In particular, the barium particles are not distributed homogeneously inside the cell. This can be explained by the fact that macrophages phagocytize the particles. Thus, they should be located in vesicles in the cytoplasm. Regions without particles could correspond to the position of the nucleus, where no vesicles are expected. In contrast, osmium tetroxide stains all lipids in a cell, which leads to a very homogenous stain. This can be useful if the internal structures of the cell are going to be investigated by tomography. However, due to its toxicity, an osmium stain is not suitable for experiments with living cells.

\subsubsection{Towards in-vivo x-ray imaging of macrophages}

To study cells in a situation that is closest to their natural environment, we use the ibidi flow-chambers as a liquid chamber, where macrophages are surrounded by PBS and adhere to the plastic surface. This enables the simultaneous observation of cells with the on-axis microscope and the x-ray beam during the measurement at the GINIX instrument (see Fig. 6.3). In the previous section, we have already seen that barium sulfate particles are not homogeneously distributed within a cell. Another approach to obtain a more uniform contrast increase, which is compatible with cells being in a liquid chamber, ${ }^{1}$ is to stain the cell surface. This approach is realized by using an antibody stain as described in section 6.2 .2 , where antibodies specifically bind to the surface protein CD68, which is expressed on macrophages [75].

Figure 6.8 shows a hologram (a) of a CD68 antibody-stained macrophage with

1 Note that osmium tetroxide gave a very uniform stain. However, due to its very high toxicity, it will not be used for cells embedded in liquid environments. 


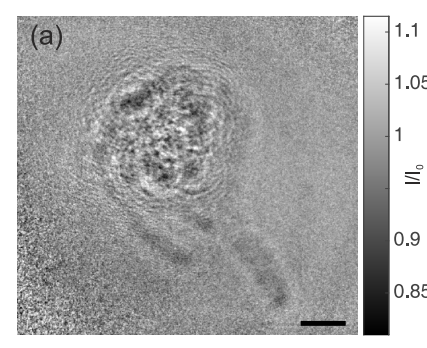

(b)

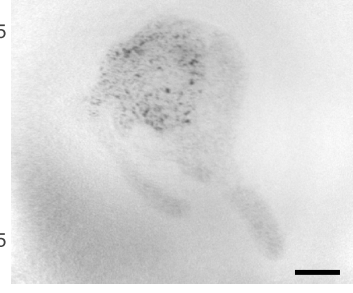

(c)

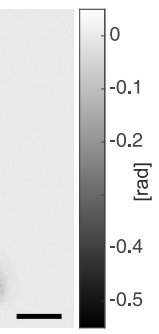

Figure 6.8: Phase-contrast imaging of a macrophage embedded in PBS with a specific CD68 antibody staining. (a) shows a normalized hologram with the usual corrections applied. (b) shows the CTF reconstruction obtained using five distances. (c) shows the HIO multi-distance phase-retrieval, which was able to compensate residual low-frequency variations. Scale bars denote $5 \mathrm{\mu m}$.

gold-enhancement together with corresponding multi-distance phase-retrievals using the CTF approach (b) and an iterative HIO reconstruction (c). In total five images at different Fresnel numbers are used. As these results are obtained with a bonded waveguide in taper geometry, the stability of the empty-beam image was not ideal. This leads to low-frequency variations in the CTF reconstruction. To ensure a proper registration of all five intensity images, the calculations are performed with preliminary phase maps obtained by single-distance CTF reconstruction, followed by a highpass and a subsequent low-pass filter. The alignment is checked by visual inspection and parameters are adapted such that no drift between the different imaging planes is observable. For the reconstruction in Fig. $6.8(\mathrm{~b})$, we choose $\kappa=10$ and $\alpha_{2}=0.1$. Of course, choosing a smaller $\kappa$ ratio would reduce the amplification of low frequencies and thus yield a more uniform background. However, this will affect the quantitative phase values significantly and will introduce halo-like artifacts around the cell. For the HIO reconstruction shown in Fig. 6.8(c), soft projections with $D=0.02$ are used. The support of the cell was manually determined in the CTF reconstruction. The HIO reconstruction produces a plausible reconstruction of the macrophage, where also the low frequencies seem to be correctly reconstructed. Surprisingly, the low-frequency variations in the background do not influence the reconstructed phase distribution. Rather, they are attributed to a non-constant amplitude of the object. In comparison to the barium sulfate stain, a more uniform increase of the contrast is achieved that is generally weaker than the contrast created by the barium particles. Additionally, pseudopodia of the macrophage can be observed quite well and some structures inside the cell were labeled stronger than the average. However, 

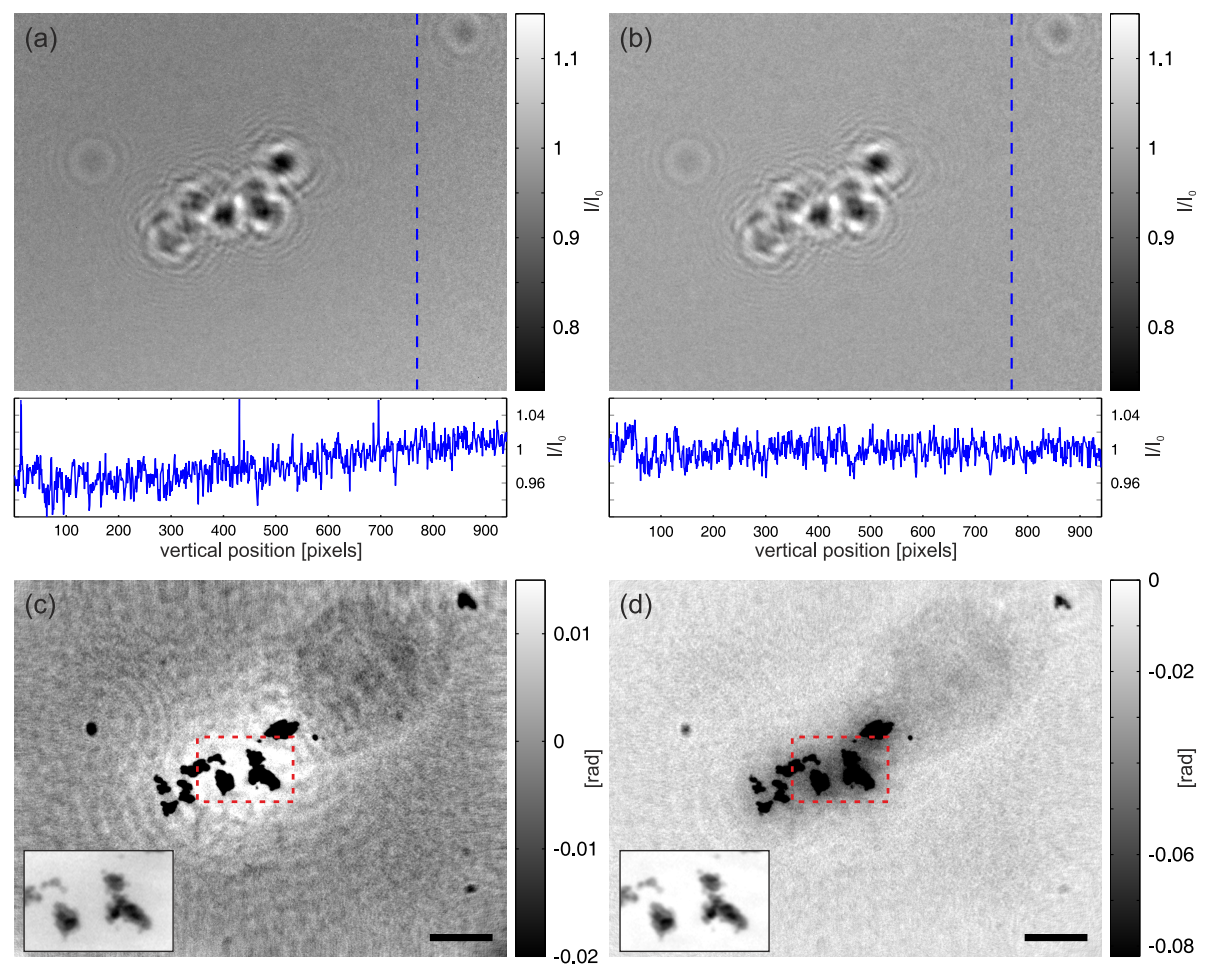

Figure 6.9: Phase-contrast imaging of macrophages embedded in PBS during the process of phagocytosis of barium sulfate particles. (a) shows the emptybeam corrected hologram, where a profile along the line plotted below. (b) shows the same hologram after applying the profile correction. (c) shows the CTF reconstruction obtained from eight distances and (d) shows a reconstruction using a multi-distance AP with positivity constraints for $\delta$ and $\beta$. In (c) and (d) the inset shows the region marked by the rectangle with the contrast adjusted to the much stronger barium particles. Scale bars denote $5 \mathrm{\mu m}$.

to distinguish between a labeling of surface structures or structures inside the cytoplasm, tomography or at least projections recorded at different angles would be necessary.

Figure 6.9(a) shows one of eight holograms used for the reconstruction of a bariumsulfate stained macrophage, which was chemically fixed in an ibidi chamber. Some hot pixels and a slight background variation can be observed in particular at the profile along the blue line. These artifacts are removed by the application of the usual correction algorithms, as can be seen in Fig. 6.9(b). Figure 6.9(c) and (d) show corresponding phase-maps reconstructed using the single material CTF 
approach and the alternating projections approach (AP), described by Eq. (2.50), with positivity of $\delta$ and $\beta$ as an object plane constraint. While the holograms show mainly contrast due to the barium particles, both phase reconstructions reveal the cell body, which is located to the top right of the barium particles. This cell was probably fixed during the process of phagocytizing the barium particles.

For the CTF reconstruction, we chose $\kappa=10$ and $\alpha_{2}=10^{-5}$. The reconstruction assumes the object to consist of a single material, which is obviously not true for barium sulfate and unstained cell material. Thus, a typical halo around the strong barium particles can be observed, if the contrast is adjusted to the weak phase shifts of the cell body as done in Fig. 6.9. These typical halo artifacts were already observed in the simulations used for Fig. 2.6. One way to overcome these artifacts is the use of phase-retrieval algorithms that use less restrictive constraints. Several trials to use a (combined) Holo-TIE approach failed here, which we attribute to the signal to noise ratio in the images. ${ }^{2}$ Also the Holo-TIE approach with multidistance fitting, described in section 2.2.3.2 did not accomplish the reconstruction of the cell. Only the AP algorithm can deliver a reconstruction that shows the weak cell body as well as the strong barium particles. Hard projections are used and the algorithm is stopped after 5000 iterations, which takes several hours if no resampling of the images is used. It has to be mentioned that the structure of the cell body was not visible in significantly less iterations and that the rigorous normalization of the background is a key point to maintain the sensitivity in the AP algorithm. By contrast, the robustness of the CTF-based phase-retrieval is demonstrated once again, as it reveals the cell body even if uncorrected intensities as in Fig 6.9(a) are used.

The ultimate test to see if the approach is compatible with in situ or even with in vivo conditions is to try the imaging of (initially) living cells. To this end, cells are cultivated in an ibidi chamber with $\mathrm{CO}_{2}$ independent medium and transported to the beamline in a mobile incubator, which keeps the cells at $37^{\circ} \mathrm{C}$. During the experiment, the cells are observed with the on-axis microscope. Discrimination between living and dead cells can thus be made by observing their adhesion state, i.e., dead cells fail to adhere to the plastic surface and fall off due to gravitation. The microscope images are used to align an unstained cell and a cell labeled with barium sulfate in the x-ray beam. Figure 6.10 shows holograms (left) and corresponding CTF reconstructions (right) of the unstained (Fig. 6.10(a,b)) and barium labeled (Fig. 6.10(c,d)) macrophages, which were alive shortly before the measurement started. Apart from being a proof of concept that living cells can be

\footnotetext{
${ }^{2}$ Note that a single-distance CTF reconstruction also did not show the weak cell body.
} 
observed, this experiment demonstrates the contrast increase by the barium sulfate for cells embedded in a liquid environment. In the case shown here, an about 20fold increase is observed. For the reconstruction in Fig. 6.10(b), a total dose of 633 Gy distributed over 3 distances was used to image the unstained cells. For the barium labeled cells shown in Fig. 6.10(c,d), the dose could be even decreased to 106 Gy. In both cases a density of typical cell material $\left(\rho=1.35 \mathrm{~g} / \mathrm{cm}^{3}\right)$ is assumed for dose calculations, which may be underestimating the actual dose in the case of stained cells. The cells can be clearly identified with and without the contrast agent and the inset in Fig. 6.10(d) shows the lower cell with adjusted contrast, to enhance the visibility of the underlying, unstained cell body.

In comparison to other phase-retrieval techniques like ptychography, the necessary dose for propagation-based nearfield holography measurements is much lower $[16,184]$. Motivated by this fact, we recently demonstrated the application of holo-
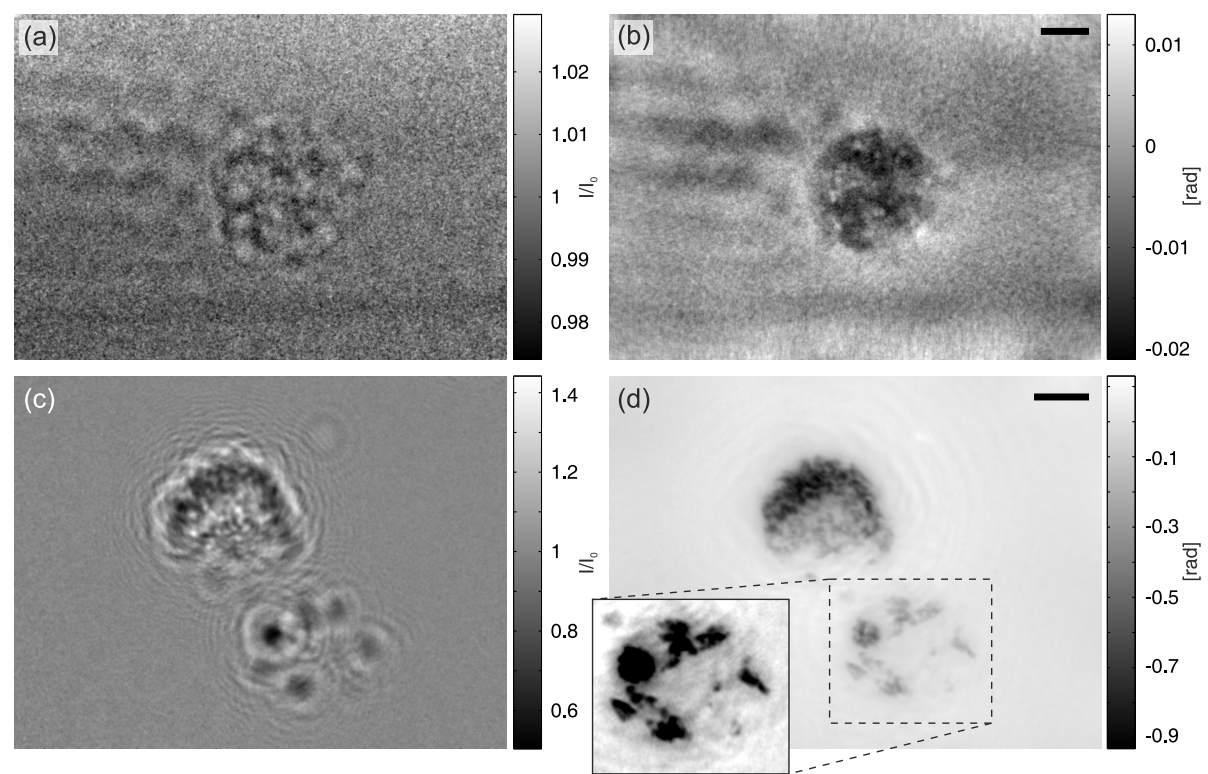

Figure 6.10: Phase-contrast imaging of living macrophages. The left column shows holograms obtained from (a) an unlabeled macrophage and (c) two barium-sulfate labeled cells. The right column shows the CTF retrieved phase maps (b) for holograms as shown in (a) using 3 distances and (d) for holograms as in (c) using 5 distances. The inset in (d) shows the lower cell, where the contrast was adjusted to enhance the weak signal of the cell next to the barium particles. Scale bars denote $5 \mu \mathrm{m}$. 
graphic phase-contrast imaging using x-ray waveguides on live bacteria [16]. Using the modified HIO algorithm for single-distance phase-retrieval, a dose-dependent density tracking was enabled that revealed a biological reaction of the cells to the radiation .

\subsection{D imaging of single macrophages}

To unambiguously discern surface structures and features that are located inside a cell, a projection image that shows an overlay of all the structures is not enough. If phase retrieval is successfully performed for holograms recorded under different viewing angles, the resulting phase maps can be used to perform a tomographic reconstruction as described in detail in chapter 3. However, if nanoscale resolution is wanted, the mechanical stability of the rotational positioner has to be very high. Additionally, the sample may change over time, especially if it is embedded in a liquid environment. In the following sections, we will briefly introduce a method to correct for motion artifacts, which will often hamper the reconstruction of fine details, followed by an application of holographic phase-contrast tomography to single macrophages.

\subsubsection{Algorithmic motion correction}

\subsubsection{Vertical correction}

If a sample is rotated around the vertical $z$-axis, a movement in $z$-direction is not expected. However, caused by mechanical instabilities or by drifts in the sample this movement may still occur. A suitable method to correct vertical motions is given as follows: All measured projections are integrated in y-direction, i.e., perpendicular to the rotation axis and to the x-ray propagation direction. The integration will yield $1 \mathrm{D}$ profiles $l_{\alpha}(z)$ for every rotation angle $\alpha$. If the resulting profiles are mapped in a color-plot depending on the $z$-coordinate and the rotation angle $\alpha$, a so-called linogram is obtained. Figure 6.11(b) to (d) show several linograms for uncorrected and corrected data of a cell in a capillary. To increase the signal to noise ratio (SNR), the integration can be carried out only at the position of the cell.

The linogram in Fig. 6.11(b) shows strong variations mainly caused by vertical motion of a cell embedded in agarose. To correct the motion, the shift-dependent deviation $d(s)$ between a reference profile with angle $\alpha_{1}$ and a profile under the 
angle $\alpha_{2}$, vertically shifted by $s$, is defined by

$$
d(s)=\int_{z}\left|l_{\alpha_{1}}(z)-l_{\alpha_{2}}(z-s)\right|^{2} \mathrm{~d} z
$$

This deviation can be calculated for several values of $s$ and by numerical minimization of $d(s)$, the optimal shift value $s\left(\alpha_{2}\right)$ can be determined for every angle $\alpha_{2}$. A recursive refinement can be used to achieve the necessary accuracy. If projections of compactly supported 3D objects that fit in the FOV are used, every profile

(a)
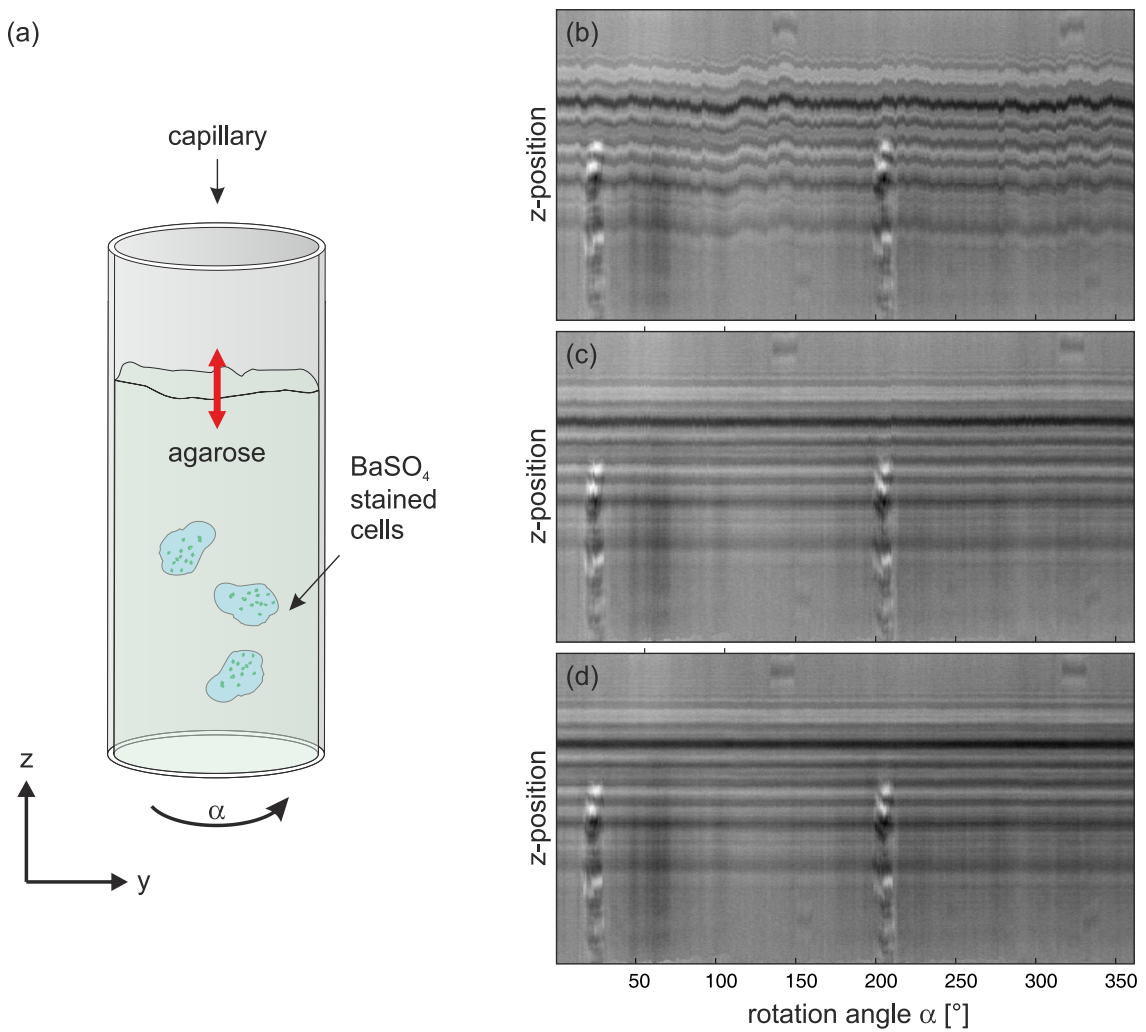

Figure 6.11: (a) Sketch of the experimental situation, in which the main motion artifacts are caused by vertical movements of the surrounding agarose gel. (b) Linogram, where no corrections have been applied. As every profile should be on the same height, these images can be used for vertical motion alignment. (c) shows a linogram, where alignment was performed using the phase-maps. (d) shows a linogram, where alignment was performed with the unreconstructed holograms. 
should be the same. Thus, an arbitrary angle or the average of many profiles can be used as a reference, to which all other profiles are aligned. However, for interior tomography this procedure does not work anymore. For the case shown in Fig. 6.11 , limiting the $z$-integration range to the upper third can significantly improve the alignment. If the range is not limited, structures outside the region of interest disturb the registration, which in this case are present e.g., in the the lower part of the linogram. We also observed that the alignment of the unreconstructed holograms, leading to the linogram in Fig. 6.11(d), is more robust than using the CTF reconstructed phase maps, which leads to the linogram shown in Fig. 6.11(c). This effect may be explained by the fact that low-frequency variations, which are enhanced by phase retrieval, dominate the deviation values $d(s)$ and thus slightly wrong shifts are calculated.

\subsubsection{Horizontal correction}

The alignment in $y$-direction is generally more challenging, as structures in the projection naturally move during a rotation around the $z$-axis. For compactly supported objects, Helgason and Ludwig derived consistency conditions for the Radon transform $[69,101]$. One of those conditions states that the $k$-th moment of a projection can be described by $k$ Fourier components. In particular, this means that for $k=0$ the mass has to be conserved and for $k=1$ the center of mass in $y$-direction $\left(\mathrm{COM}_{y}\right)$, defined by

$$
\mathrm{COM}_{y}(\alpha)=\int p(\alpha, y) \cdot y \mathrm{~d} y
$$

follows a sine curve [93]. For a holographic projection of a pure-phase object, one problem is that the COM cannot unambiguously be calculated, as the integration over dark and bright interference fringes will cancel out. Especially if residual beam variations are present, they will heavily influence the values of the COM. If many efforts are put into the phase-retrieval step and every projection can be automatically reconstructed using a modified-HIO algorithm with support constraint, the COM alignment is able to deliver good results.

An alternative approach is to use a reprojection alignment [130] as sketched in Fig. 6.12. To this end, a preliminary 3D dataset $f^{(i)}$ is reconstructed using the projections $p_{\alpha}^{(i)}$. If the data is heavily misaligned, the volume $f^{(i)}$ may be smoothed prior to the reconstruction, to first correct coarse motions. This 3D volume is then reprojected, to yield synthetic projections $p_{\text {repr. }}$, which may not represent the object but share a common rotation axis without motions artifacts. 


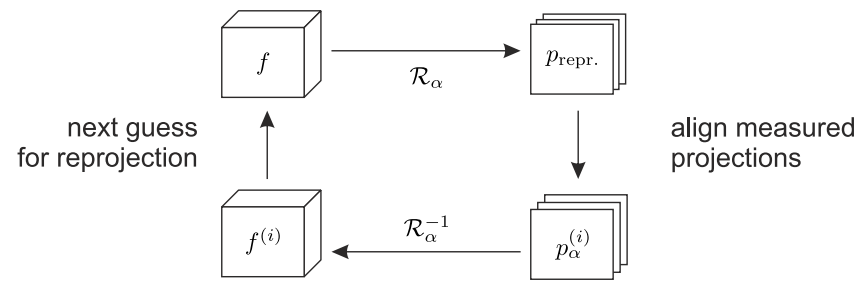

Figure 6.12: The idea of reprojection alignment: Starting with the measured projections $p_{\alpha}^{(i)}$, a $3 D$ volume $f^{(i)}$ is reconstructed. Depending on the strength of the misalignment, the reconstruction is smoothed to create a $3 D$ guess $f$ that is used to calculate reprojected measurements $p_{\text {repr. }}$. A next guess $p_{\alpha}^{(i+1)}$ is obtained, by aligning the measured data to the reprojections.

These reprojections can be used as a reference, to align the measured projections $p_{\alpha}^{(0)}$ to the reprojected ones. The whole procedure can be repeated iteratively by using the aligned projections to obtain another $3 \mathrm{D}$ guess. However, in most cases the first iteration already reduces most of the artifacts if the misalignment is not too strong. Note that even if COM alignment is successful, a few iterations of reprojection alignment may still improve the reconstruction.

\subsubsection{Stained macrophages in aqueous gels}

Figure 6.13 shows measured data of a barium sulfate labeled macrophage that was embedded in agarose gel in a capillary. Several holograms are recorded during a rotational scan and one of them is shown in Fig. 6.13(a). Detailed parameters are listed in Table 6.2. For this and for all following tomographic reconstructions, all projections are enlarged by applying replicative padding of their boundary values, to avoid artifacts caused by interior reconstruction. The holograms are normalized as before by calculating profiles at all four edges. The CTF-based phase-retrieval is performed using Eq. 2.29 with $\alpha_{1}=8 \cdot 10^{-4}$ and $\alpha_{2}=0.08$. The phase map obtained from the hologram shown in (a) can be seen in Fig. 6.13(b).

Due to the special kind of sample preparation (see Fig. 6.11(a)), a strong vertical motion is introduced during the tomographic measurement. The $\mathrm{x}$-ray is attenuated in the $500 \mu \mathrm{m}$ thick agarose gel, which may lead to a temperature increase that is able to locally melt the agarose. As the remaining agarose block is confined by the capillary wall, it can move in the vertical direction by a few micrometers. A sinogram of the uncorrected data is shown in Fig. 6.13(c), in which significant 
distortions of the sine curves can be seen. These motions can also be clearly identified in the linogram shown in Fig. 6.11(b). If the shifts are compensated by minimizing the deviations described by Eq. (6.7), a smooth linogram is obtained (cf. Fig. 6.11(d)). To reduce any drifts in the horizontal direction, 5 iterations of iterative reprojection-alignment are applied. The resulting sinogram is shown in Fig. 6.13(d), where obviously a strong improvement can be observed as the sine curves now appear much smoother.

Figure 6.14 shows resulting reconstructed slices for the dataset presented in Fig. 6.13. In the reconstruction obtained from CTF-reconstructed projections without any motion correction (Fig. 6.14(a)), the barium particles are blurred and slight streak artifacts can be observed. Using the same projections with prior motion-
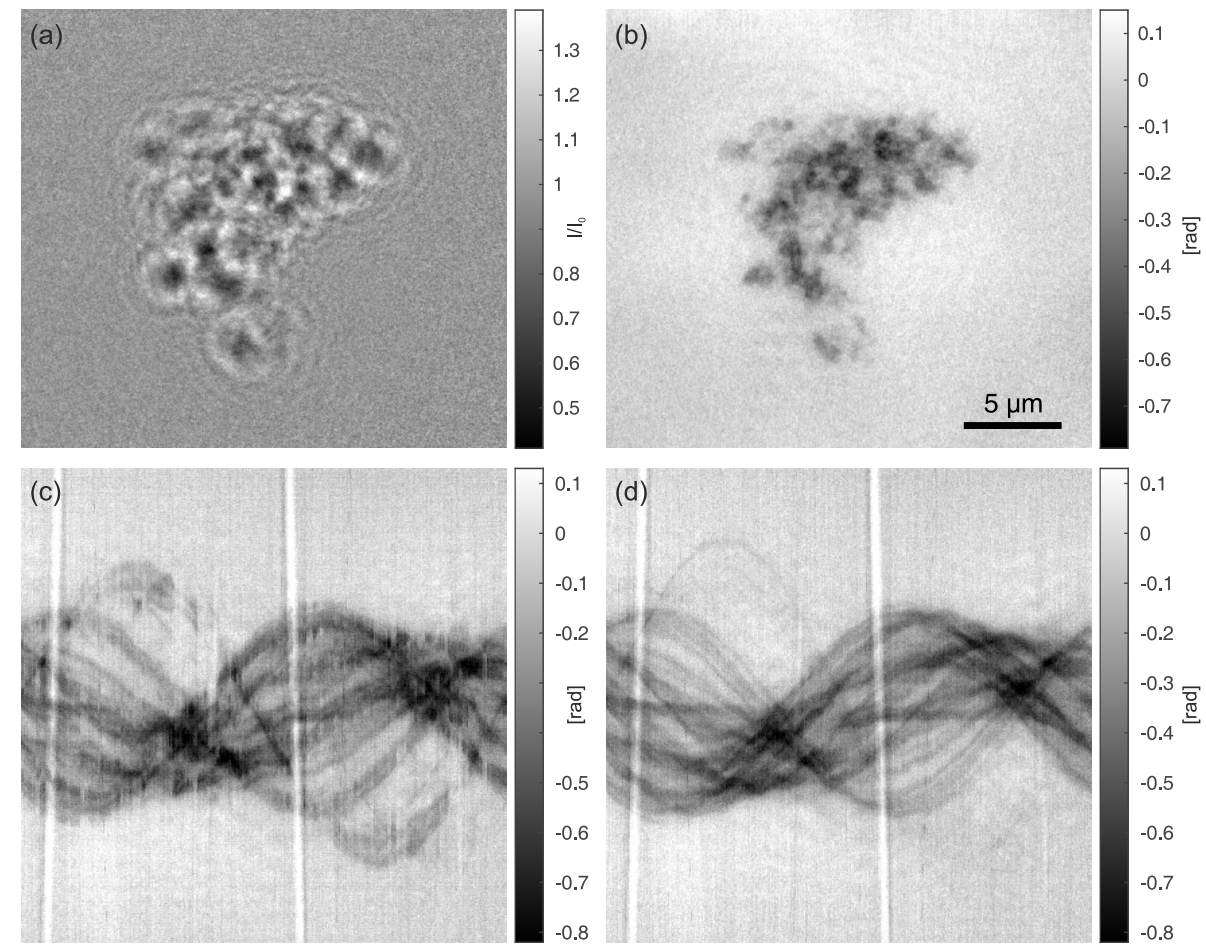

Figure 6.13: (a) Hologram of a barium labeled macrophage embedded in agarose gel inside a capillary. (b) shows the corresponding CTF reconstruction using a single distance. (c) Sinogram of the tomographic measurement, without any motion alignment. (d) Sinogram after applying the vertical linogram alignment and 5 reprojection iterations. 


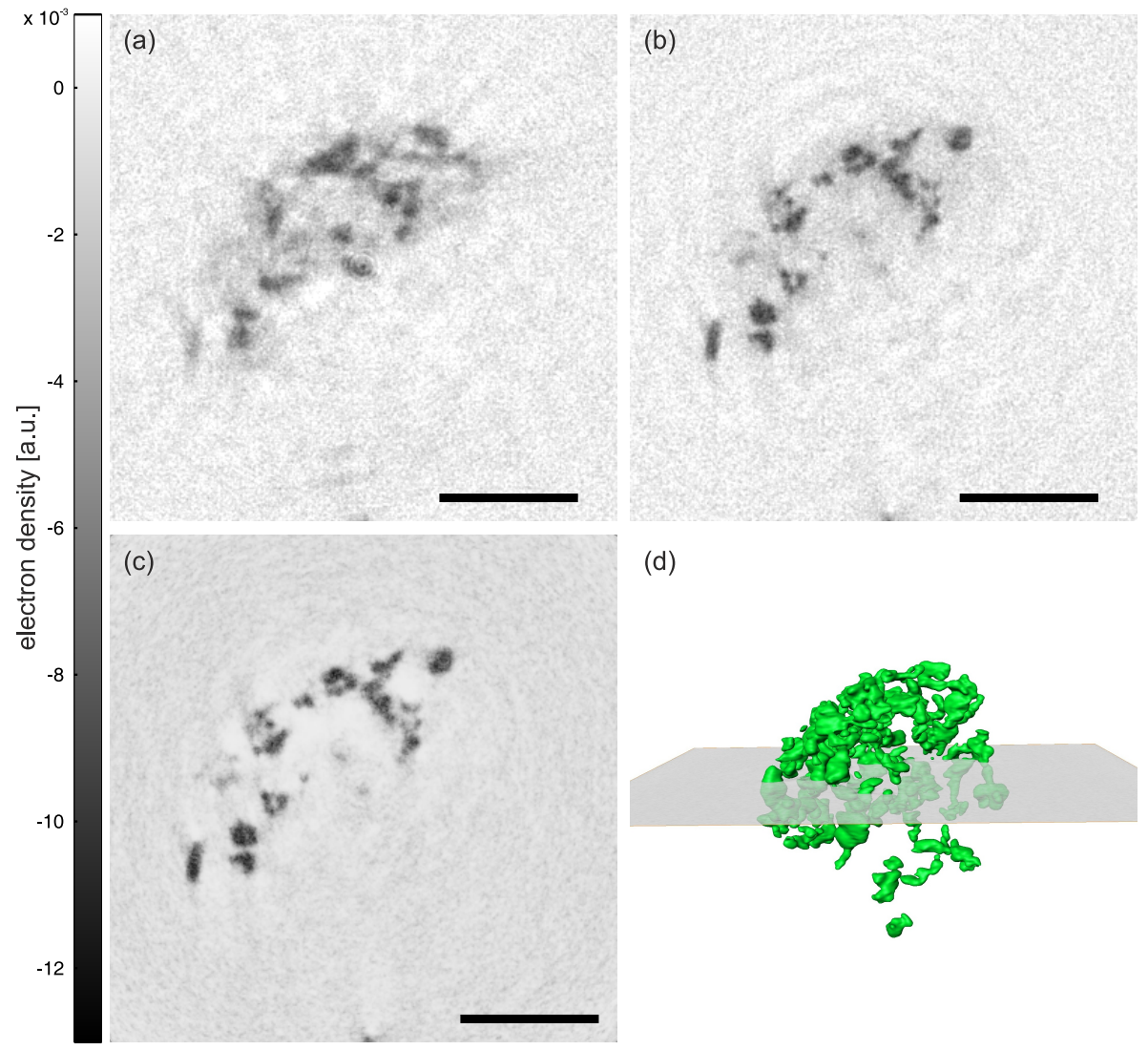

Figure 6.14: The influence of motion correction to reconstructed orthoslices. (a) FBP reconstruction of CTF reconstructed phase-maps from data without alignment (b) FBP reconstruction of the same projections after the full alignment procedure. (c) IRP reconstruction after the full alignment was applied to the measured holograms. (d) $3 D$ rendering of the results shown in (c). Scale bars denote $5 \mu \mathrm{m}$. 
correction applied, an orthoslice for the same position is reconstructed, as can be seen in (b). The barium particles appear significantly sharper with increased contrast and reduced streak artifacts. Unfortunately, no structure of the unstained cell body can be observed as the SNR is not high enough.

As only a single-distance is available for this dataset, the CTF-based phaseretrieval will be not the best choice. However, during the measurement some dirt, located on the outside of the capillary, traverses the images during rotation. This hinders an HIO reconstruction, as a 2D support constraint cannot be applied. However, the iterative reprojection phase-retrieval (IRP), which combines phase retrieval with the $3 \mathrm{D}$ reconstruction, is perfectly suited to be applied here. To this end, the shift information from the alignment is used to correct the measured holograms and a $3 \mathrm{D}$ volume is directly reconstructed using the update formula given by Eq. (2.57). With this approach, the tomographic consistency is implicitly used as an additional constraint [151], which leads to a better tomographic reconstruction, shown in Fig. 6.14(c). In particular the SNR is improved, which enables the observation of some smaller barium particles.

Figure $6.14(\mathrm{~d})$ shows a 3D rendering of the automatically segmented bariumsulfate particles in green together with an $x y$-orthoslice. For the segmentation, the $3 \mathrm{D}$ histogram of the IRP reconstruction is thresholded. The outline of the particle arrangement indicates the shape of the cell body, which however cannot be directly observed as the SNR is still not high enough to observe the very weak contrast of the unstained cell body.

\subsubsection{Cells embedded in resin}

\subsubsection{Osmium stained}

The $2 \mathrm{D}$ projections could already indicate that the use of osmium tetroxide as a homogenous stain for the cells increases the contrast of macrophages significantly. In order to investigate if internal 3D structures of cells can be analyzed in a large piece of surrounding material using phase-contrast tomography, we start by studying osmium stained macrophages, which are embedded in a a block of plastic (from a Petri-dish) and epon, with a total diameter of about $2 \mathrm{~mm}$ (see Fig. 6.2). Figure 6.15 shows a typical hologram (a) of osmium stained cells, embedded in such a plastic/resin block, which was recorded at the ID16a beamline. The reconstructed phase map is shown in (b), where four different distances have been used. The regularizing parameters were chosen to $\kappa=15$ and $\alpha_{2}=0.01$. In contrast to the $2 \mathrm{D}$ results shown before, this block was not polished so that the 
surface structure heavily dominates the projection images. However, there are still some cells that can be recognized in the projections, marked by the red arrows in Fig. 6.15(b). Some of these cells are aligned in the center of rotation and a tomographic scan is performed, with detailed parameters shown in Tab. 6.2. Note that the rough-surface structure disturbs quantitative values of the reconstructed phase map, as no proper normalization can be performed and the parameters were chosen to optimize the appearance of the cells in the $3 \mathrm{D}$ reconstruction.

As no waveguide illumination was used to obtain the holograms at the ID16a beamline, artifacts due to the empty-beam correction can be observed in particular at the edges of Fig. 6.15(a). The use of several defocus distances reduces the artifacts as the object changes its magnification during the movement along the optical axis while the artifacts stay in place, so that they will be averaged in the reconstructions. However, this procedure only reduces the artifacts in the reconstructed phase-map and does not fully remove them. The stripes will lead to ring artifacts in tomographically reconstructed orthoslices, as illustrated in Fig. 6.16(a). Although internal structure in the cells can be observed, the ring artifacts impede an automatic segmentation. To remove the ring artifacts, the wavelet based ring-removal (see section 3.2.1) is used, where the first 4 components of Daubechies 24 tap wavelets are filtered with $\sigma=3$. The resulting orthoslice is shown in Fig. 6.16(b), revealing a considerable reduction of rings. In particular,
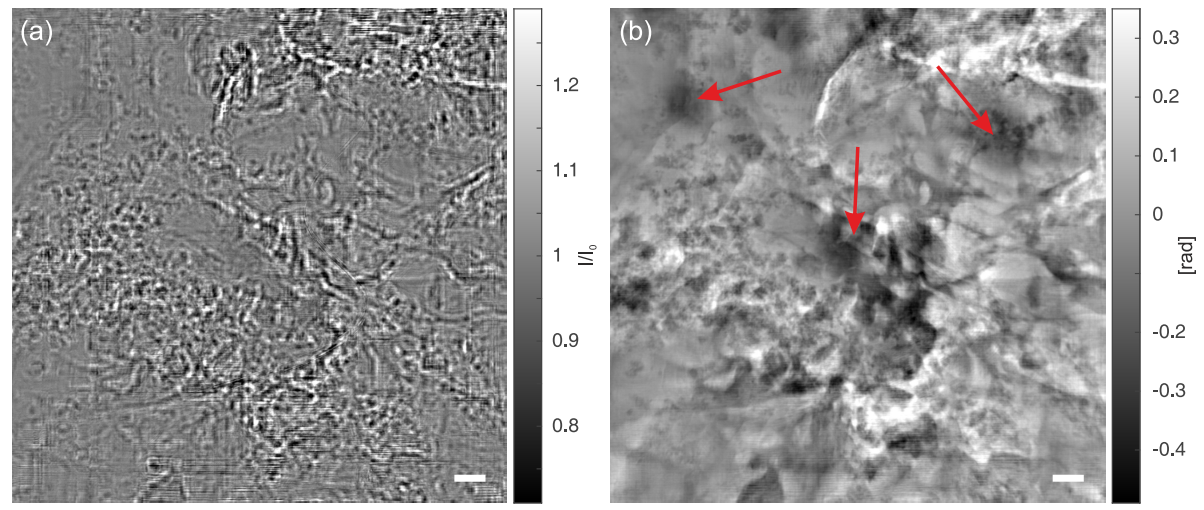

Figure 6.15: (a) hologram of osmium-stained macrophages embedded in a nonpolished resin block with (b) the corresponding four-distance CTF-based phaseretrieval. Note that the surface structure of the epon/plastic block dominates the image. The arrows mark positions where the outline of some cells can be observed. Scale bars denote $5 \mathrm{\mu m}$. 


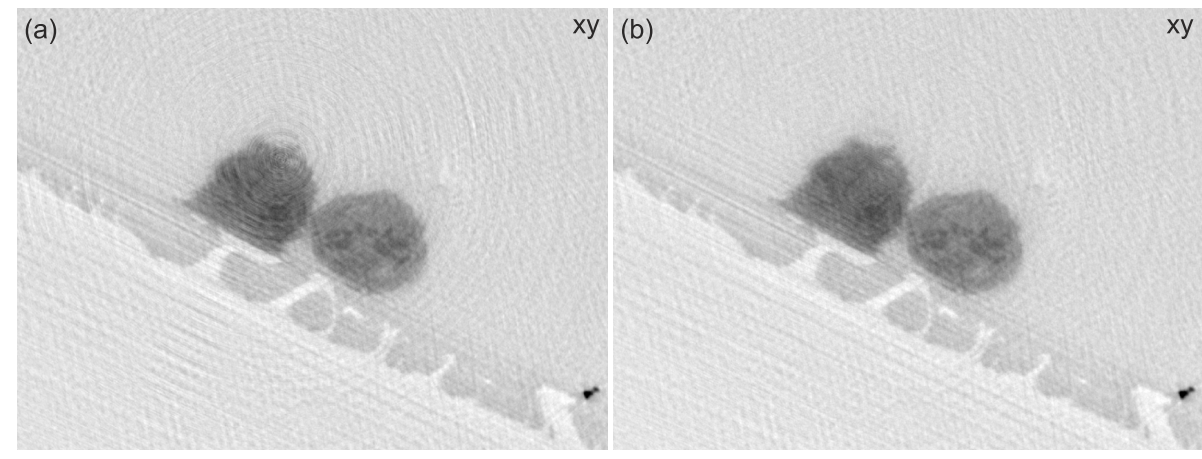

Figure 6.16: Influence of wavelet based ring-removal presented in [115]. (a) shows an orthoslice perpendicular to the rotation axis, where many ring artifacts are present due to a failing empty-beam correction. (b) shows the same orthoslice after applying the wavelet based ring-removal.

the features inside the cell can be better isolated, which enables the automatic histogram-based segmentation.

Figure 6.17(a) shows a tilted slice through the 3D volume, which is oriented such that it is oriented parallel to the Petri-dish surface. Thus, the slice shows a crosssection of many cells with their different internal structures. To automatically segment all cell bodies, the volume is resampled by a factor of 2 in each direction and a thresholding of the 3D histogram, followed by erosion and dilatation operations is performed. Only regions that contained more than 50000 connected voxels are kept and identified as cells. The internal structure, which can be identified in the orthoslices, is automatically segmented for selected cells. To this end, another histogram-based segmentation is performed, where the region is limited to the region of single cells that were segmented before.

Figure $6.17(\mathrm{~b})$ shows a side view of the tilted slice from (a) together with a 3D rendering of the segmented cells in half-transparent blue and internal structures of selected cells in red. Additionally, an orthoslice in the $y z$-plane is present for orientation. Many single cells can be clearly identified which show complex internal structures. For example, the inner structure of the cell marked by the arrow suggests the hypothesis that the cell was fixed during the process of mitosis, as it shows a generally weaker density with compactified regions that may contain the chromosomes.

A single macrophage is marked by a dashed rectangle in Fig. 6.17(a) and (b), for which the $y z$-orthoslice is shown in (c). Based on their density, the Petri-dish and 


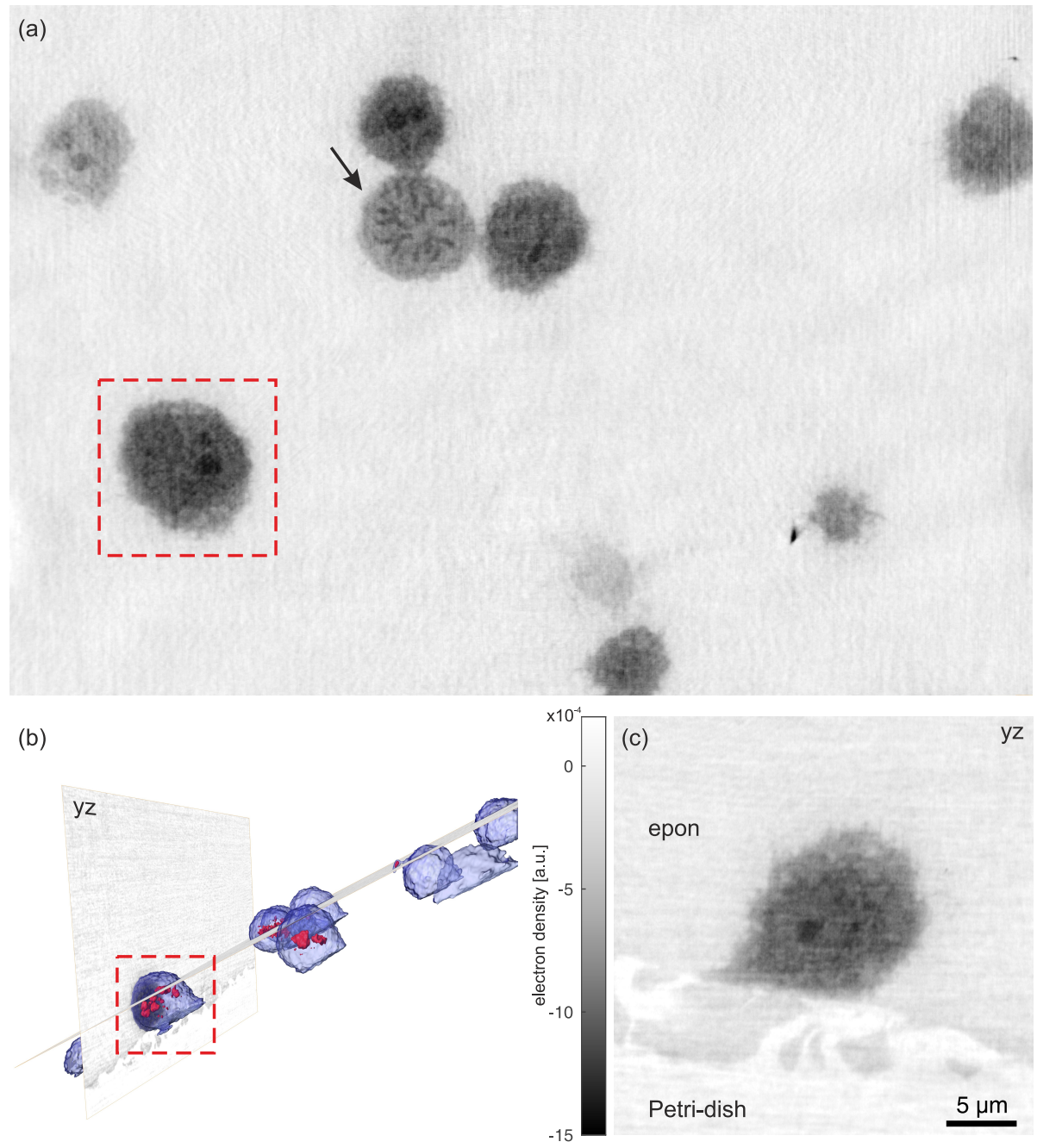

Figure 6.17: $3 D$ reconstruction of the osmium stained cells. (a) shows a tilted slice through the reconstructed $3 D$ volume that lies parallel to the Petri-dish surface and thus hits most of the cells. An arrow marks a cell, which shows different internal structures, maybe due to the cell being fixed during the process of mitosis. (b) shows the position of the slice in (a) relative to an yz-orthoslice. (c) shows a zoom to an yz-slice perpendicular to the rotation axis that shows the cell marked by the dashed rectangle in (a) and (b). 


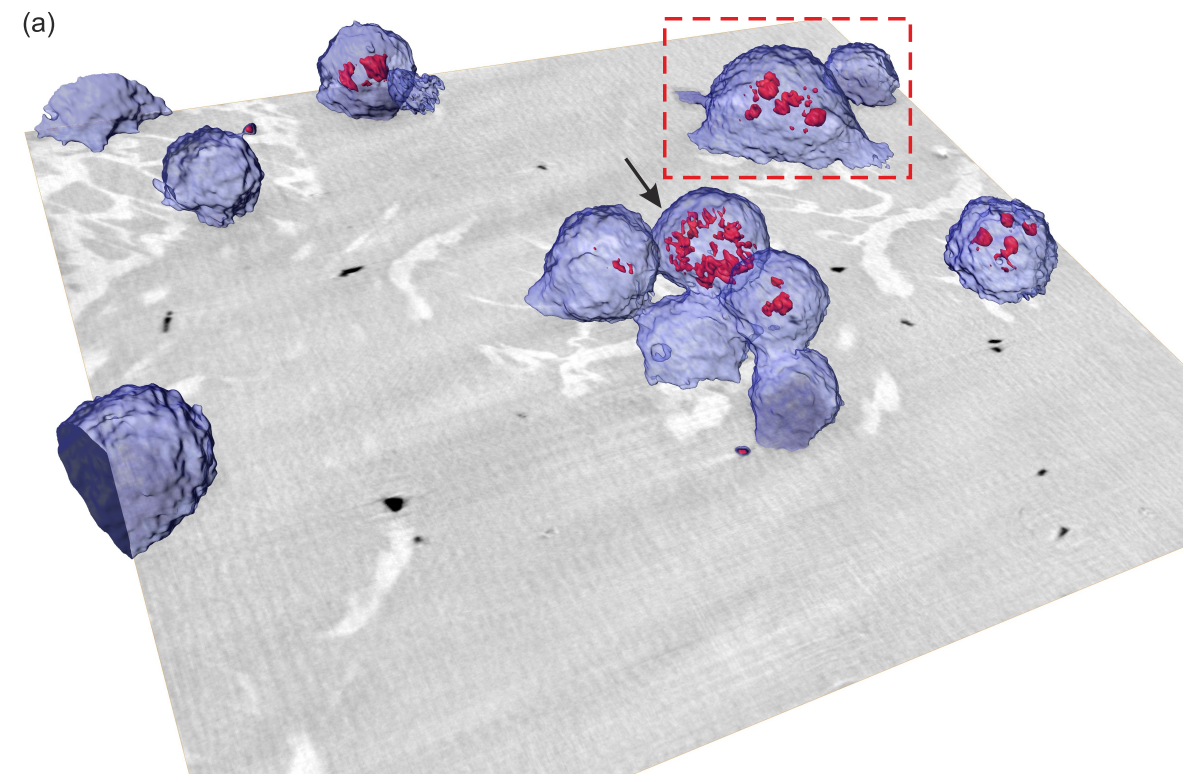

(b)

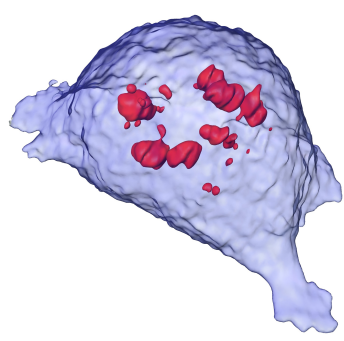

(c)

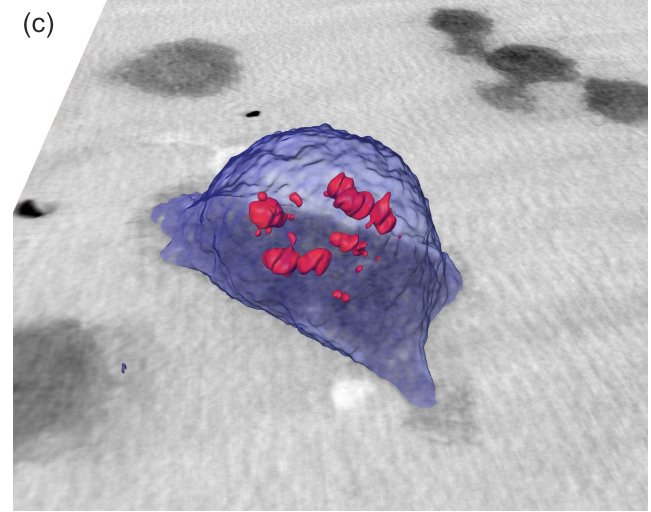

Figure 6.18: Osmium stained macrophages. (a) overview of the $3 D$ rendering of the dataset shown in Fig. 6.17 and 6.15. The cells are automatically rendered using a histogram-based segmentation. The internal structure for selected cells is automatically segmented, followed by erosion and dilatation processes and finally rendered in red. An arrow marks the same cell as in Fig. 6.17. (b) and (c) show an isolated close up of the cell marked by the dashed rectangle, where some pseudopodia can be observed. 
the (denser) epon can be distinguished. It can be clearly seen that the single cell adheres to the Petri-dish by stretching out its pseudopodia. Figure 6.18(a) shows another $3 \mathrm{D}$ rendering of the same volume, in which the tilted slice is shifted such that the transition between epon and the Petri-dish with all the cells on it can be observed. The cell from Fig. 6.17(c) is again marked by a dashed rectangle. Figure 6.17(b) and (c) show a close up of the 3D rendering of the cell, in which some pseudopodia of the cell, stretched in several directions, can be clearly identified. Additionally, some internal, sphere-like structures can be observed that may be large vesicles, which were stained stronger than the average.

\subsubsection{Unstained cells}

To investigate the sensitivity of the method, we next tried to obtain $3 \mathrm{D}$ reconstructions of unstained macrophages. To this end, a scan of unstained cells in a plastic/resin block is performed. A holographic projection and its corresponding single-distance CTF reconstruction are shown in Fig. 6.7(a) and (b). Parameters for the tomographic scan are listed in Tab. 6.2. A Fourier-filter based ring-removal is applied as described in section 3.2.1.

Figure 6.19(a) shows a reconstructed orthoslice of the 3D dataset. As in Fig. 6.17, the rough surface-structure of the Petri-dish can be observed as a region

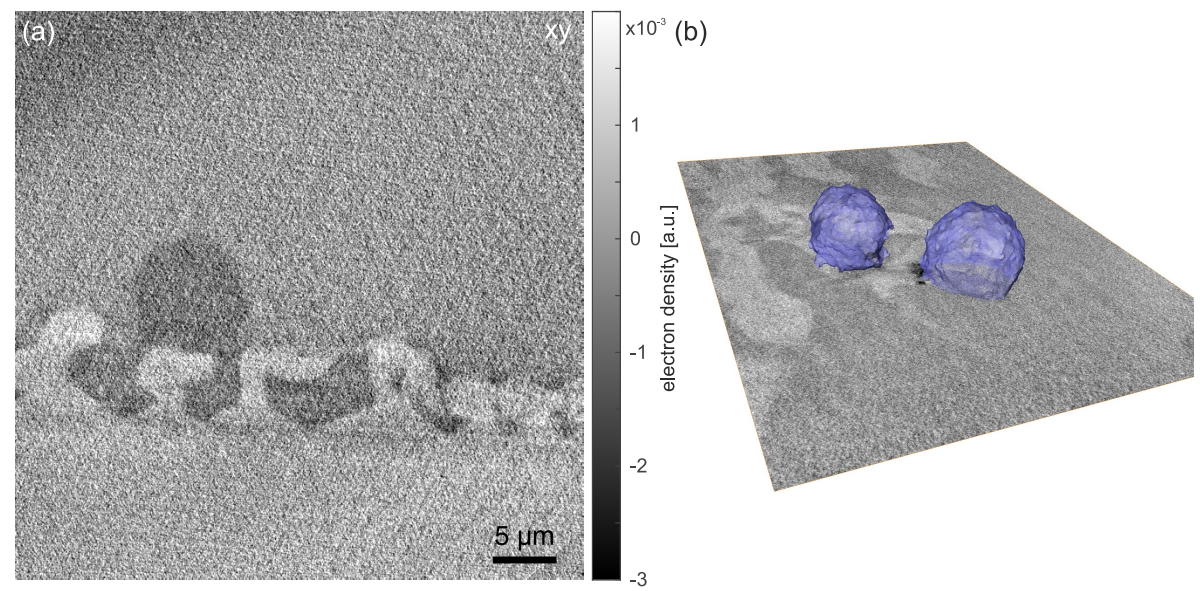

Figure 6.19: Tomography results of unstained macrophages. (a) reconstructed orthoslice perpendicular to the rotation axis, in which a cell can be observed on the rough surface of the Petri-dish plastic. (b) $3 D$ rendering of two cells, which were automatically segmented in a $4 \times$ resampled dataset. 
with lower density, next to the epon which has a higher density. Located on the Petri-dish surface, a single macrophage can be recognized, which shows a very low SNR. To automatically segment the cell body, the SNR is increased by resampling the volume by a factor of 4 , and the cell region is segmented using the magic-wand tool of the rendering software Avizo. Regions far below and above the resin/plastic transition, which obviously do not belong to the cell, are removed and the remaining structures are displayed as half-transparent blue surfaces in Fig. 6.19 (b). Note that in contrast to the osmium-stained cells, no internal structures can be observed.

\subsubsection{Osmium and barium stained}

In Fig. 6.14 we could see barium-sulfate particles inside a macrophage cell. However, only the barium particles could be observed, while structures of the surrounding cell-body could not be identified. To learn something about the 3D location of barium sulfate particles inside macrophages, we use barium-labeled macrophages embedded in epon. Additionally, all lipids are stained with osmium to enhance the contrast of the cell body. A typical hologram and a CTF reconstructed phase map are shown in Fig. 6.7(e) and (f). Already the phase-reconstructed projection enables the observation of barium particles inside the stained cell body, which can be clearly resolved.

A tomographic scan is performed, with parameters listed in Tab. 6.2. Figure 6.20 (a) shows a reconstructed orthoslice perpendicular to the rotation axis. No ring removal was applied during the reconstruction process. The less dense Petridish can be seen at the bottom, while the denser epon is located above. A single macrophage can be clearly identified, with many barium-sulfate particles inside. As in Fig. 6.14, streak artifacts around the particles can be observed that are caused by motion artifacts. The application of the linogram alignment method and a few reprojection-iterations enable the reduction of the artifacts, as can be seen in Fig. 6.20(b). Figure 6.20(d) shows the sinograms used to obtain the reconstructions in (a) and (b), without and with motion-correction, respectively. Note that for this sample, more structures outside the field-of-view disturbed the alignment procedure. In particular, due to the rectangular form of the plastic/resin block, the outer edges can be seen in some projections. To this end, we only corrected projections in an angular range of $\alpha \in[0, \ldots, 137.5,236.3, \ldots, 314]^{\circ}$ for the reconstruction in Fig. 6.20(b). Projections at other angles are not changed, as otherwise additional artifacts are introduced. 


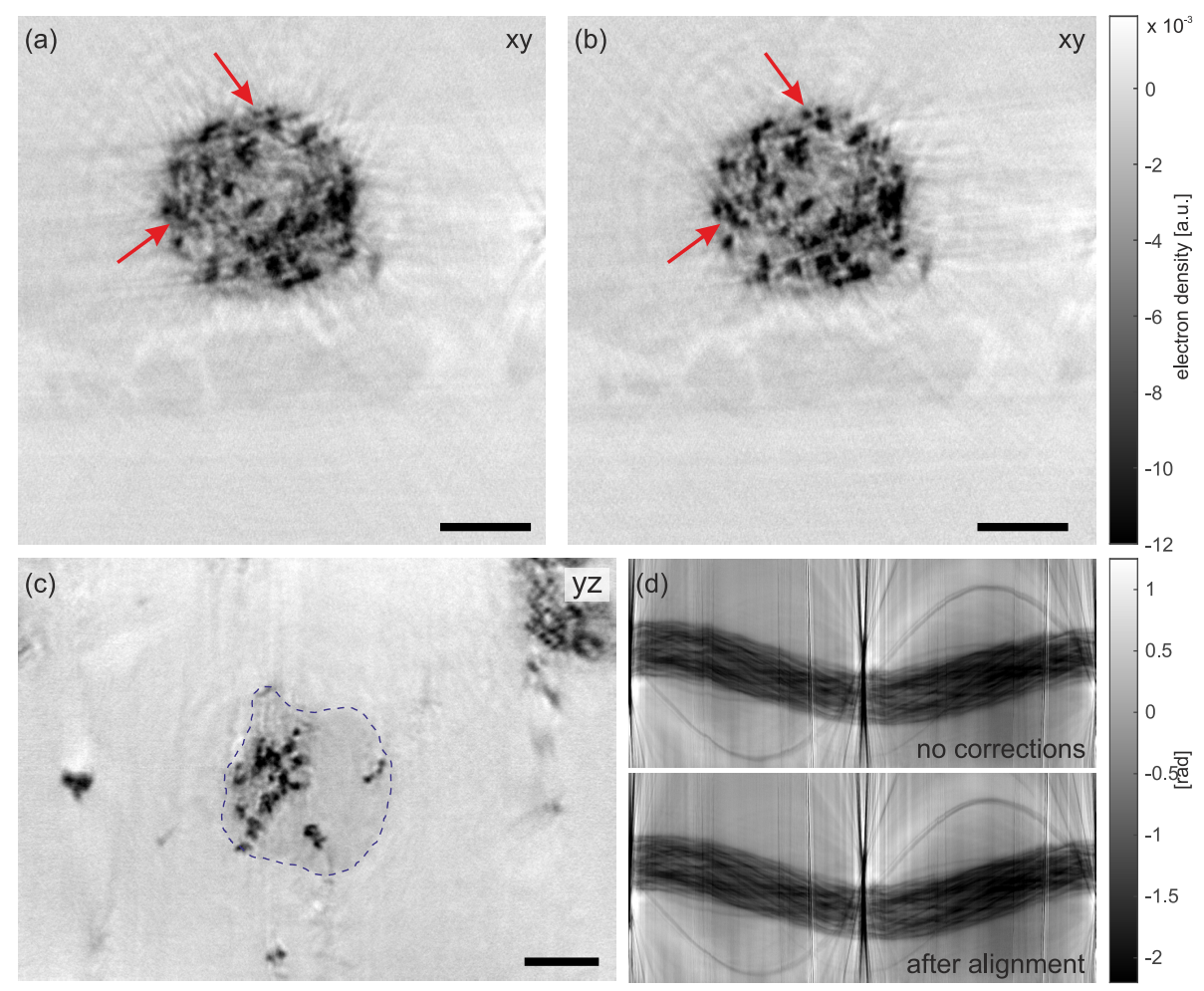

Figure 6.20: $3 D$ reconstructed data from macrophages stained with barium and osmium, where projections are shown in Fig. 6.7(e) and (f). (a) shows an $x y$-orthoslice perpendicular to the rotation axis without movement correction. (b) shows the same orthoslice as in (a) but with linogram and reprojection alignment applied. Some artifacts can be reduced, which is visible in particular at the position of the arrows. (c) shows an yz-orthoslice, i.e., perpendicular to the $\mathrm{x}$-ray propagation direction. The dashed line marks the outline of a cell body that was used for segmentation. In (d) the sinogram before and after the motion correction are compared. All scale bars denote $5 \mathrm{\mu m}$. 


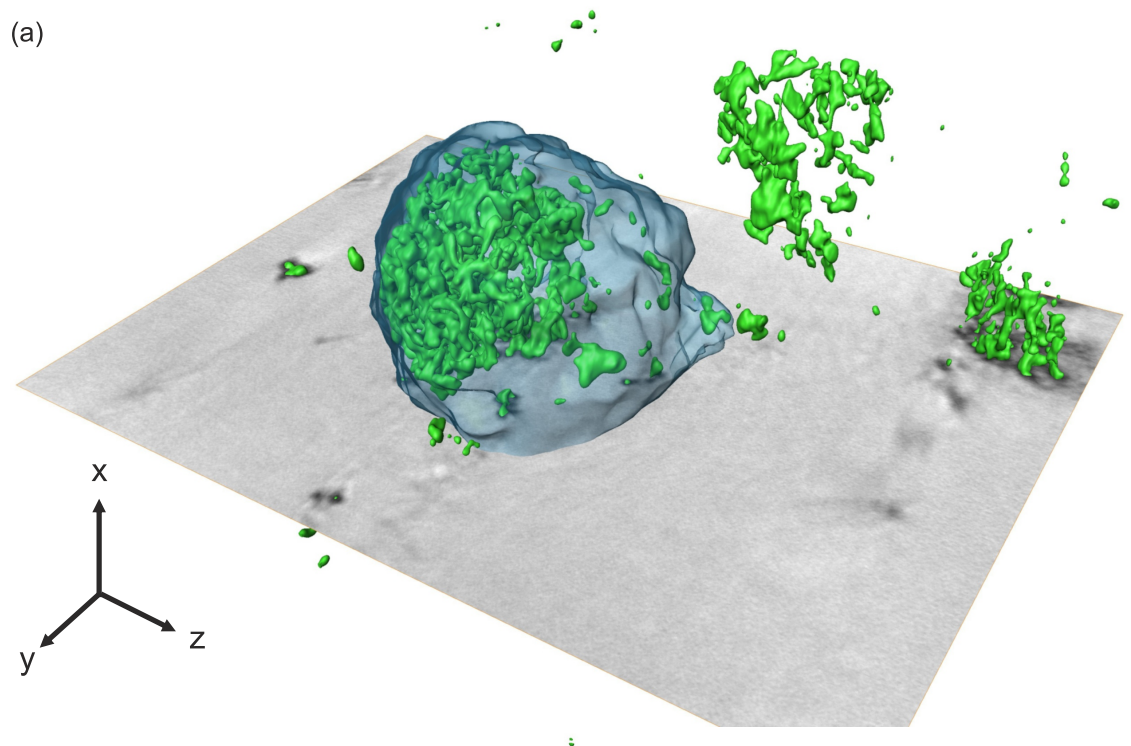

(b)

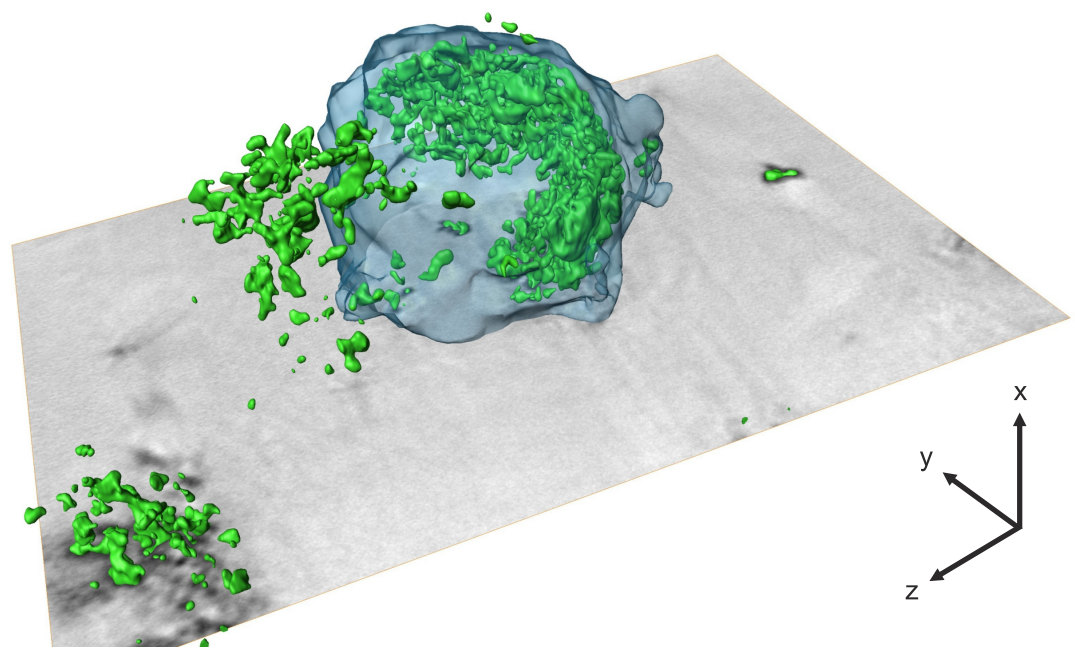

Figure 6.21: $3 D$ renderings of the data shown in Fig. 6.20 from two different viewing angles. Barium particles are automatically segmented and rendered in green, where the semi-manually segmented cell outline (see Fig. 6.20(c)) is rendered in half-transparent blue. 
Even after the (partial) motion correction, streak artifacts can be still observed. However, the quality of the reconstruction could be significantly improved. In particular the barium particles are not as blurred as in the uncorrected slice, which can be seen e.g., at the positions of the arrows in Fig. 6.20(a) and (b). An orthoslice in $y z$-direction, shown in (c), shows less artifacts as it corresponds to the direction that is visible in the projections. Additionally, the structure of the cell body can be observed next to the barium particles. The cell body is semimanually segmented, using a coarse manual segmentation, followed by refinements using the blow-tool, implemented in Avizo. This tool enables the contrast-based growing of a manual selection in the image. Thus, the selection is based on the reconstructed density instead of just the visual, subjective selection made by the user. Barium sulfate particles are automatically segmented using a thresholding of the histogram.

Figure 6.21 shows two different views of the same 3D rendering of the motion corrected dataset. The orthoslice in in Fig. 6.20(c), which is oriented parallel to the Petri-dish surface, is displayed for orientation, too. The segmented volume of barium-sulfate particles is rendered in green next to the semi-manually segmented cell body in half-transparent blue. It can be clearly seen that the barium particles are not homogeneously distributed within the cell. Rather, they seem to concentrate in half-ring-like shapes at the edge of the cell. Calculations with the segmented regions reveal a total cell volume of $V_{\text {cell }}=234.4 \mu^{3}$ while the barium sulfate inside the cell requires a volume of $V_{\mathrm{BaSO}_{4}}=15.7 \mu^{3}$. Thus, about $6.7 \%$ of the cell volume are filled with the contrast agent, which leads to a relative increase in the total cell weight of about $22.3 \%$, if a densities of $\rho_{\mathrm{BaSO}_{4}}=4.5 \mathrm{~g} / \mathrm{cm}^{3}$ and $\rho_{\text {cell }}=1.35 \mathrm{~g} / \mathrm{cm}^{3}$ are assumed.

\subsection{Resolution of the $3 \mathrm{D}$ reconstructions}

To estimate the resolution of 3D reconstructions, the Fourier shell correlation (FSC) can be used as described in section 1.5. Figure 6.22 shows two FSC curves of the IRP reconstruction of the cell, embedded in agarose in a capillary (Fig. 6.14) and of the osmium-stained cell embedded in epon (Fig. 6.18). For the calculation, two independent datasets are reconstructed, one for all projections under even angles and one for the odd angles. The intersection of the FSC with the 1/2-bit threshold curve determines the resolution of the full dataset, using all angles [176]. The FSC in Fig. 6.22(a) never reaches the 1/2-bit threshold. This means that the pixel size was not small enough to see the transition to the noise 
(a)

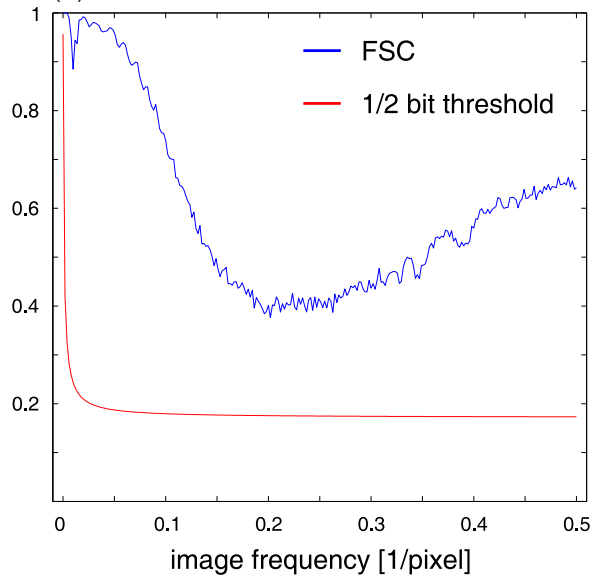

(b)

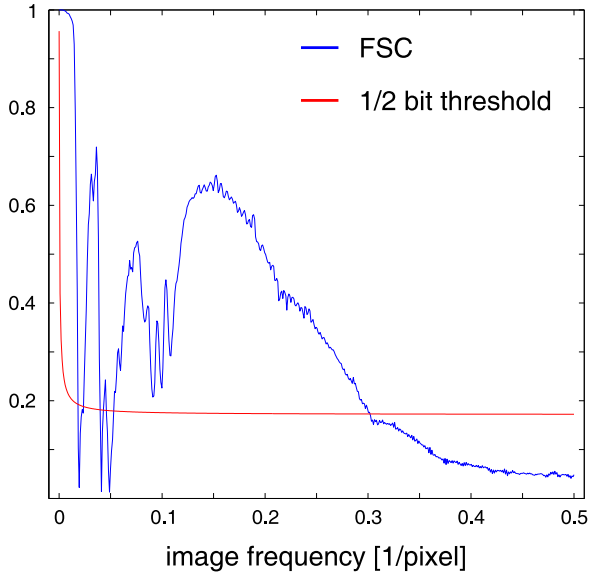

Figure 6.22: Fourier shell correlations of (a) the IRP reconstruction of the barium labeled macrophage in a capillary, shown in Fig. 6.14 and (b) the osmium stained cell embedded in a plastic/resin environment, shown in Fig. 6.18. As in (a) the correlation is always higher than the threshold, the resolution is given by the (binned) pixel size of about $51 \mathrm{~nm}$. The crossover in (b) at 0.3 periods/pixel corresponds to a half-period resolution of $83 \mathrm{~nm}$.

level. Rather, the chosen size of the pixels limits the resolution. As the projections for the IRP reconstruction were resampled by a factor of 2, a single-pixel resolution of about $51 \mathrm{~nm}$ seems plausible, especially if one keeps in mind that a motion correction was performed in the dataset without resampling and that for unbinned 2D images, a resolution below 2 pixel could be achieved. For the FSC in (b), images are not resampled. An unexpected drop at distinct low frequencies can be seen, which can be explained by the fact that the projection images contained much structures caused by the surface roughness of the sample that are not quantitatively reconstructed. The cells and their internal structure are composed mainly of higher spatial frequencies and as they could be resolved these drops can be ignored for resolution considerations. Rather, at about 0.3 periods/pixel a plausible intersection with the threshold curve can be observed, corresponding to a half-period resolution of $83 \mathrm{~nm}$. 


\subsection{Summary}

In this chapter we have demonstrated the application of propagation-based phasecontrast imaging for the $2 \mathrm{D}$ and $3 \mathrm{D}$ imaging of macrophages. The influence of different staining methods to the image contrast has been investigated and 2D projections with a resolution below $50 \mathrm{~nm}$ could be observed on dried cells using a waveguide-based illumination at the GINIX setup. Stained and unstained macrophages could be visualized in resin and in aqueous environments that enabled the observation of living cells.

To obtain 3D information about the single cells, tomography was successfully realized and reconstructions of stained and unstained cells could be retrieved, revealing sub-cellular internal structures and the detailed location of barium-sulfate particles inside macrophages. By applying motion correction algorithms and a combined $3 \mathrm{D}$ phase-retrieval algorithm, a high resolution reconstructions down to $50 \mathrm{~nm}$ is realized for barium-sulfate labeled macrophages in a liquid environment. For unstained macrophages and cells stained with osmium tetroxide no motion correction was performed as no major artifacts disturbed the reconstructions. One reason for this may be that no high resolution markers with a good contrast, as the bariumsulfate particles, were present. Hence, eventually present spurious motions may not be detected, which could blur the appearance of internal structures.

Future experiments should target in the exploration of different contrast agents that can be used to specifically mark interesting regions inside the cell to allow the answering of biomedically relevant questions or to obtain a homogenous stain like that given by osmium tetroxide, which is compatible with in vivo conditions. 


\section{Tracking macrophages in the mouse lung}

In chapter 5 we have applied phase-contrast tomography with a laboratory source, to investigate structures in the mouse lung with a resolution of about $5 \mu \mathrm{m}$. By using synchrotron-radiation sources, holographic phase-contrast measurements with a higher resolution are possible, which enabled the observation of sub-cellular structures in single macrophages. On single cells, we have reached a 3D resolution down to $50 \mathrm{~nm}$, and even a relatively large sample diameter of up to $2 \mathrm{~mm}$ did not limit the reconstruction quality. The next step is to combine both approaches, to resolve single cells with sub-cellular resolution in large pieces of medically relevant samples. In this chapter, we will use thick-tissue slices of mouse lungs, in which macrophages are labeled with barium-sulfate to investigate the precise location of macrophages and their internal structure in the lung. Parts of this chapter have been published as [89], whereas this thesis contains some additional details about the experimental methods and results.

\subsection{The medically relevant question}

In 2011 about 235 million people suffered from asthma and it is the most common chronic disease among children [188]. Allergic asthma is thought to be caused by complex and incompletely understood environmental and genetic interactions [105]. Although macrophages are known to be involved in processes of allergic inflammation [114], their exact role in asthma is still not well understood [10, 11, 190]. In particular, migration properties of macrophages may have significant effects in asthma [110] and a question that has to be answered is, if alveolar macrophages are able to migrate from airway lumen through the epithelium.

The conventional approach to learn something about structures on the cellular level in many biomedical disciplines is to use immunohistochemical staining of thin tissue slices, followed by optical microscopy. Figure 7.1 shows typical histological slices of lung sections from asthmatic (a,b) and control mice (c,d) [104]. To enable 

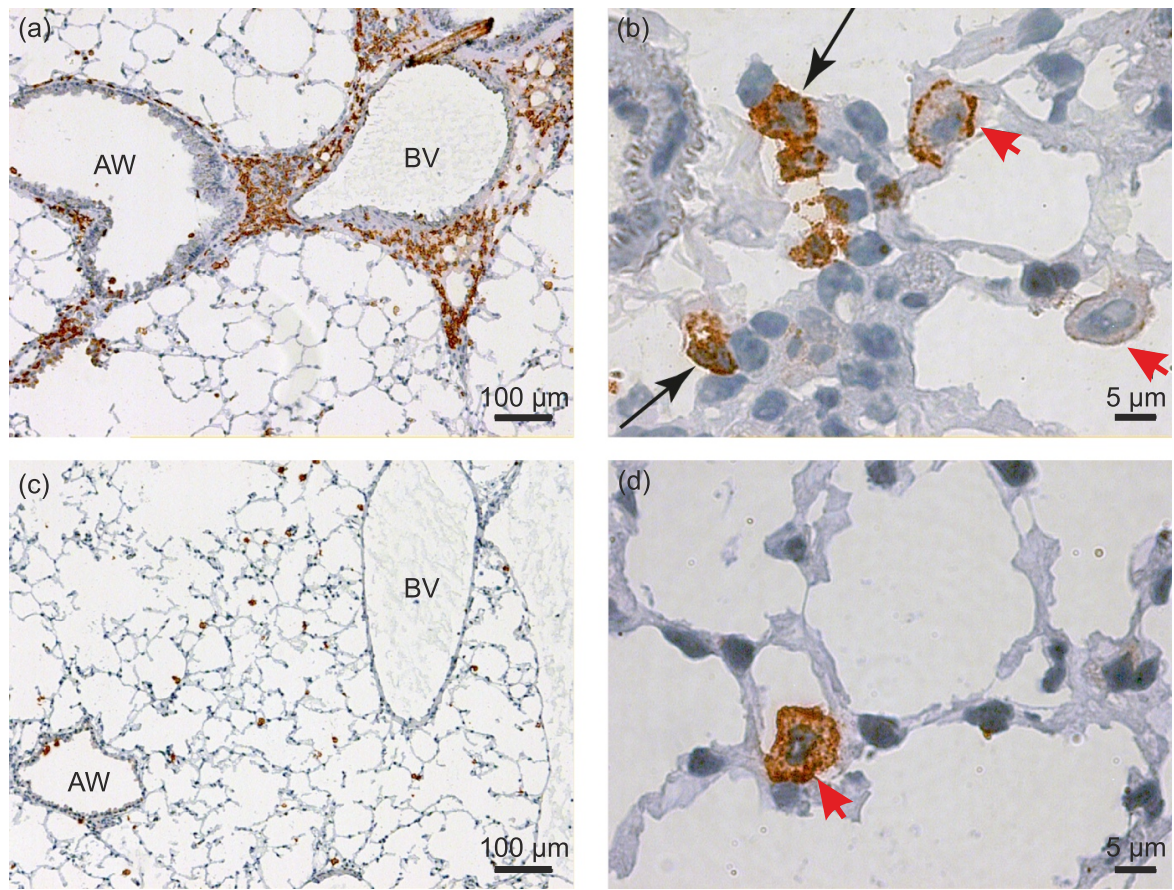

Figure 7.1: Immunohistochemical staining of cryo-frozen lung-tissue slices from $(a, b)$ asthmatic and $(c, d)$ control mice with haematoxylin/eosin and anti-Siglec$F$-antibody stain. Siglec- $F$ is a protein that is primarily expressed on the surface of certain immune cells. In asthmatic mice the infiltrated cells around blood vessels $(B V)$ and airways $(A W)$ show a high expression of the protein. In the higher resolution images shown in (b) and (d), eosinophils (long, black arrows) and macrophages (short, red arrows) can be observed. Figure reproduced from [104].

microscopic imaging and the recognizability of single cells, the whole lung is filled with an optimal cutting temperature compound and is frozen in liquid nitrogen. Subsequently $5 \mu \mathrm{m}$ thick slices are cut using cryotomes, followed by the application of many different staining reagents. Using conventional light microscopy, precise locations of the stained cells on one particular slice can be obtained, as illustrated in Fig. 7.1. If 3D tracking of cells in the context of anatomical structures is needed, all these time-consuming procedures have to be carried out for thousands of slices and mechanical distortions due to the cutting may inhibit reasonable results [181]. Recently this approach was used to obtain $3 \mathrm{D}$ reconstructions of a whole human brain, by analyzing 7404 histologically stained brain slices [4]. 
Phase-contrast tomography using cone-beam illumination offers a much simpler alternative to obtain structural information on different length scales. In this chapter we will apply holographic phase-contrast tomography to lung-tissue slices of asthmatic and control mice. By using barium-labeled macrophages, which were intratracheally injected into the living mice, the cells can be tracked in 3D in relation to the anatomical structures. Thus, the approach may enable many new applications for biomedical research.

\subsection{Sample preparation}

The macrophage MH-S cell line [107] is cultivated as described in section 6.2.1 and stained by co-incubation with $3.5 \mu \mathrm{l}$ barium-sulfate suspension per milliliter cell suspension for 4 hours (see section 6.2.2 for more details). This contrast agent concentration ensures that most cells have taken up sufficient $\mathrm{BaSO}_{4}$ for robust cell tracking.

Asthma is induced in mice by using ovalbumin (OVA) sensitization and challenges, as described in detail in [104]. Control mice are prepared that receive PBS instead of OVA. Three days after the last OVA challenge, a total of $1 \times 10^{6}$ barium-labeled macrophages suspended in $30 \mu \mathrm{PBS}$ is intratracheally instilled into the lungs of

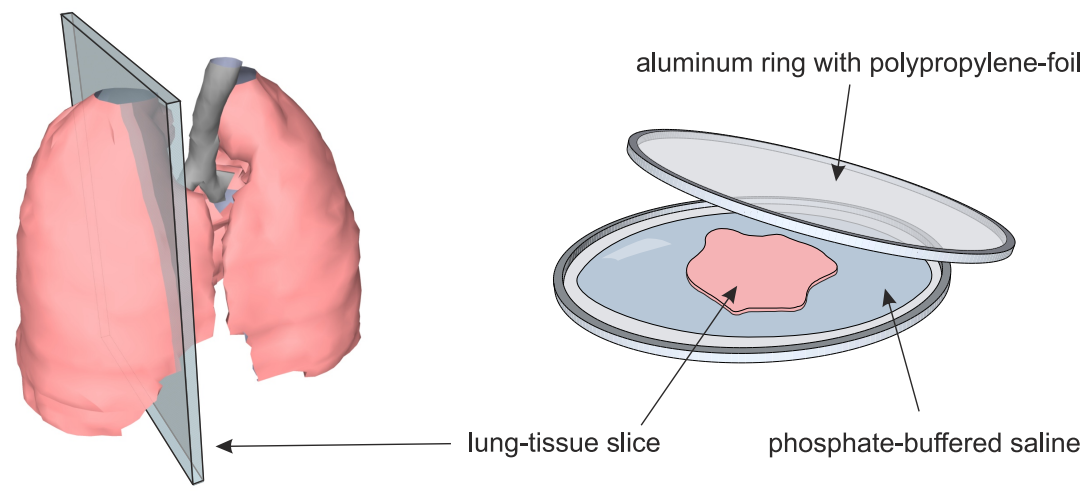

Figure 7.2: Sketch of the sample preparation: $500 \mu \mathrm{m}$ thick slices of chemically fixed lung tissue are placed into a self-built liquid chamber, consisting of two aluminum rings covered with polypropylene foil. To build the chamber a PBS reservoir is dropped on the lower foil, the slice is placed on top of it and the second foil is used to cover the sample. The chamber is sealed with glue to avoid drying. 

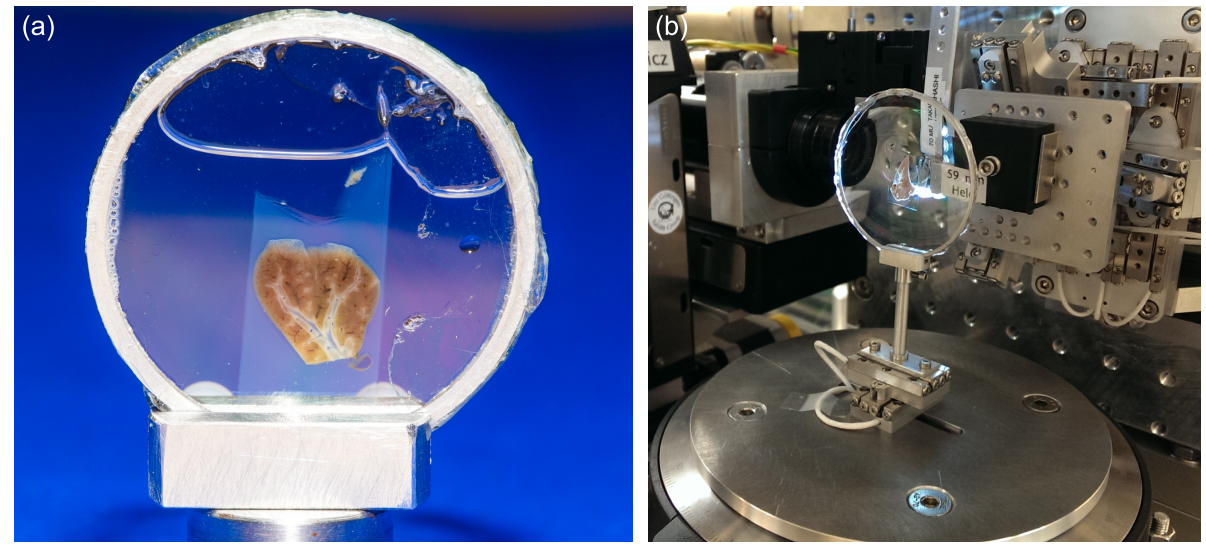

Figure 7.3: (a) Close up photograph of a prepared sample (b) Photograph of a sample mounted in the GINIX setup. Note that in (b) a larger aluminum ring was used.

asthmatic and control mice under Ketamine 10\%/Xylazine 2\% anesthesia. After 24 hours, mice are euthanized using an isoflurane overdose. The lungs are dissected, filled with $4 \%$ paraformaldehyde (PFA) to compensate the collapse of the lung and fixed in $4 \%$ PFA for 24 hours. The preparation of the mice is carried out at the UMG in strict accordance with the guidelines for the care and use of laboratory animals of the local ethics office of the UMG. After fixation, individual lung lobes are embedded in 5\% agarose gel and subsequently cut into $500 \mu \mathrm{m}$ thick slices using a Leica VT1000 S vibrating blade microtome (Leica, Wetzlar, Germany). The lung tissue slices are stored in $0.02 \%$ sodium azide/PBS solution at $4^{\circ} \mathrm{C}$ until the experiments in order to avoid bacterial degradation of the samples.

Immediately before the experiments, lung-tissue slices are placed in PBS between two polypropylene foils, as sketched in Fig. 7.2. The foils are mounted on 500 $\mu \mathrm{m}$ thick aluminum rings of either 25 or $50 \mathrm{~mm}$ outer diameter. To prevent drying during the measurements, the sample holder is sealed with two-component glue, forming a closed liquid-chamber of $500 \mu \mathrm{m}$ thickness. Figure 7.3 shows two photographs of prepared samples mounted on sample holders. Depending on the outer diameter of the aluminum rings, a different missing wedge, i.e., angular regions where the ring blocks the radiation, is present. 


\begin{tabular}{llll}
\hline & large FOV & medium FOV & high resolution \\
\hline voxel size $[\mathrm{nm}]$ & 430 & 245 & 51.5 \\
min. $x_{01}[\mathrm{~mm}]$ & 299 & 190 & 40 \\
$x_{02}[\mathrm{~m}]$ & 0.53 & 5.07 & 5.07 \\
energy $[\mathrm{keV}]$ & 17.5 & 13.8 & 13.8 \\
$F^{10}$ & 1.97 & 0.365 & 0.075 \\
illumination/beamline & $\mathrm{KB} / \mathrm{ID} 22$ & $59 \mathrm{~nm} \mathrm{WG} / \mathrm{P} 10$ & $59 \mathrm{~nm} \mathrm{WG} / \mathrm{P} 10$ \\
number of projections & 1500 & 720 & 900 \\
number of distances & 1 & 1 & 4 \\
exposure time $[\mathrm{s}]$ & 0.1 & 3 & 1 \\
total fluence $\left[\mathrm{photons} / \mathrm{\mu m}^{2}\right]$ & $1.9 \cdot 10^{8}$ & $6.9 \cdot 10^{6}$ & $2.1 \cdot 10^{8}$ \\
dose [Gy] & $5.6 \cdot 10^{4}$ & $3.0 \cdot 10^{3}$ & $9.1 \cdot 10^{4}$ \\
\hline
\end{tabular}

Table 7.1: Overview of relevant parameters for the different experiments.

\subsection{Measurements and phase retrieval}

For the measurements of the lung-tissue samples, different experimental settings are used. Thus, different near-field imaging-regimes ranging from direct-contrast to the holographic regime are present. Table 7.1 gives an overview of the detailed experimental parameters.

Experiments in the direct contrast regime are performed at the ID22 beamline, where the radiation is focused to a spot below $100 \times 100 \mathrm{~nm}^{2}$ and no x-ray waveguide was used. As no single-crystal monochromator is implemented, a high flux in the order of $10^{12}$ photons/second is present, which comes at the price of a relatively large bandwidth of $\Delta \lambda / \lambda=0.015$. To detect the x-ray radiation, a lens-coupled system is employed, where a thin single-crystal scintillator is viewed by a 10-fold objective and a 1.85-fold eye-piece. The images are digitized using a Frelon camera, resulting in a detector pixel size of $p=0.756 \mu \mathrm{m}$. The tomographic experiments are performed in continuous scanning mode, i.e., images are captured on-the-fly during the movement of the rotational positioner. Thus, the time needed to record a full tomogram at a single distance is only about 15 minutes, including the time needed to capture empty images before, after and during the scan. As this configuration was only used for the imaging of large fields of view (FOV), the Fresnel number indicates the presence of the direct-contrast regime. In the following, this experimental setting will be denoted large FOV.

Phase-contrast imaging at higher resolutions is performed with the waveguidebased GINIX setup at the P10 beamline. To this end, the radiation is focused with KB-mirrors and a crossed-multilayer waveguide with $59 \mathrm{~nm}$ guiding core is 
precisely aligned in the focal spot, resulting in an effective source size between 13 and $16 \mathrm{~nm}$. The waveguide produces a slight astigmatism, which is corrected by using different Fresnel numbers for the $y$ - and $z$-direction [14]. The total flux exiting the waveguide is about $2 \cdot 10^{8}$ photons/second. The x-ray detector was an indirect x-ray camera (Pandora), which comprises a fiber-optically coupled sCMOS camera with $p=6.54 \mu \mathrm{m}$ pixel size, equipped with a $15 \mu \mathrm{m}$ thick gadox scintillator. By changing the focus to object distance $x_{01}$, different magnifications can be chosen and experiments are performed in a medium-FOV setting with $p_{\text {eff. }}=245 \mathrm{~nm}$ effective pixel size and a high-resolution setting with about $p_{\text {eff. }}=50 \mathrm{~nm}$ pixel size.

Figure 7.4(a) shows a typical phase-contrast projection, recorded in the largeFOV setting. The sample was a lung-tissue slice of an asthmatic mouse, in which barium labeled macrophages were intratracheally injected. A photograph of this particular sample can be seen in Fig. 7.3(a). Due to the small magnification, the direct-contrast regime is present and various lung structures like fat-bubbles and single macrophages can already be recognized in the projection. In (b) the reconstructed phase distribution using the MBA approach (Eq. (2.8)) is shown, which even in the magnified inset does not differ significantly from the phase map that is retrieved by the single-distance CTF approach, shown in Fig. 7.4(c). For the reconstruction, a coupling ratio of $\kappa=200$ is assumed, where high frequencies in the CTF reconstruction were regularized with $\alpha_{2}=0.01$. To ensure the comparability between the MBA and the CTF reconstruction, a Tikhonov-type regularization (see Eq. (2.45)) is enforced for the MBA reconstruction and $\alpha=4 \pi F^{1} \kappa^{-1}$ is chosen. If very fine details are regarded, a slightly better resolution of the CTF reconstruction can be observed, confirming the theoretical expectations.

Figure 7.5 shows a typical projection in the medium-FOV setting of a lung-tissue slice of a control mouse, which had barium labeled macrophages intratracheally instilled into the lung. A photograph of the sample is shown in Fig. 7.3(b). Due to the larger detector pixel size $p$, the camera is positioned farther away, leading to generally smaller Fresnel numbers compared to the ID22 setup. Thus, the images will have a better low-frequency contrast as the holographic regime is present. Fig. 7.5(b) shows the reconstructed phase-map obtained with the MBA algorithm and (c) the CTF reconstruction based on Eq. (2.29). For both reconstructions the Tikhonov type regularization parameters are chosen to $\alpha_{1}=5 \cdot 10^{-4}$ and $\alpha_{2}=0.1$ and just a single-distance was used. The high resolution information is significantly blurred if the MBA algorithm is applied, which can be clearly seen by comparing the structure in the insets of Fig. 7.5(b) and (c). 

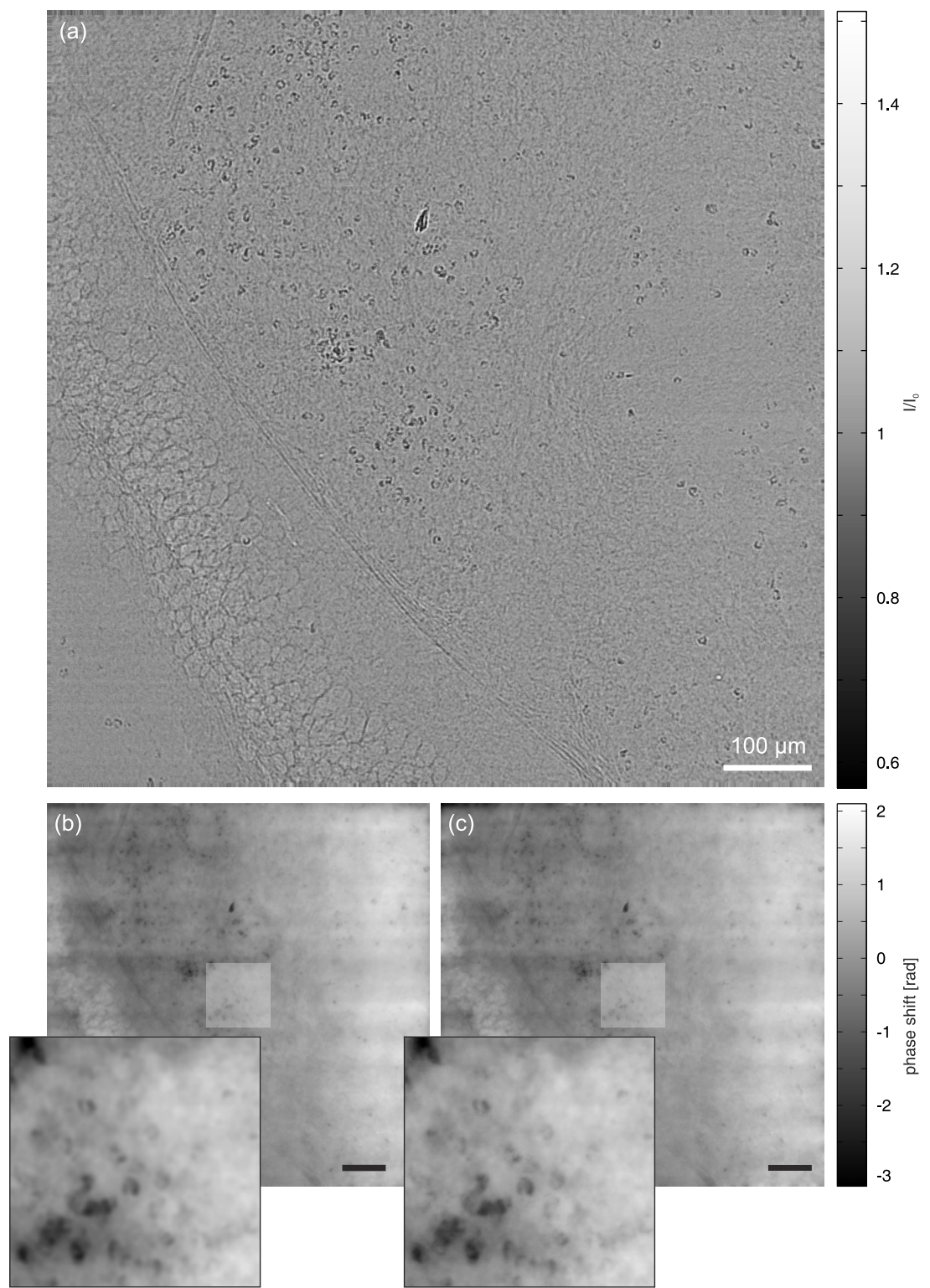

Figure 7.4: Typical projection data in the large-FOV setting: (a) Phasecontrast projection of a lung-tissue slice in the direct-contrast regime. (b) Phase retrieval of the projection in (a) using the MBA algorithm. The inset shows a magnification of the region marked by the bright rectangle. (c) Single-distance CTF-based phase-retrieval of the same projection with the same magnified inset. The round, dense structures in the inset are caused by barium-sulfate present in macrophages. Scale bars denote $100 \mu \mathrm{m}$. 

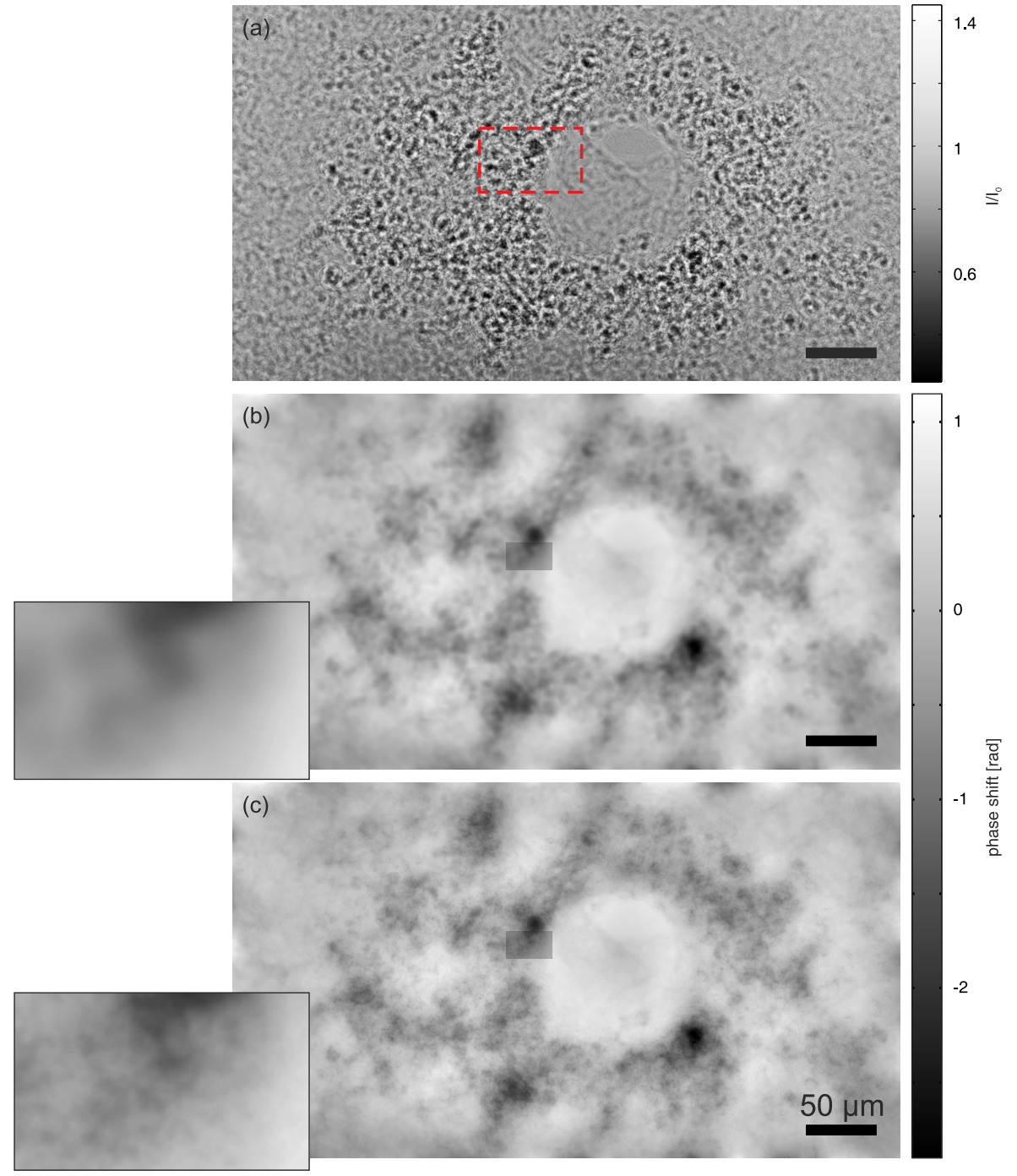

Figure 7.5: Typical projection data in the medium-FOV setting. (a) Holographic phase-contrast projection of a lung-tissue slice. (b) Reconstructed phase distribution of (a) using the SMO algorithm. (c) Reconstructed phase distribution using the CTF approach. The region marked by the dark rectangle in (b) and $(c)$ is shown as a magnified inset, respectively. Scale bars denote $50 \mu \mathrm{m}$. 


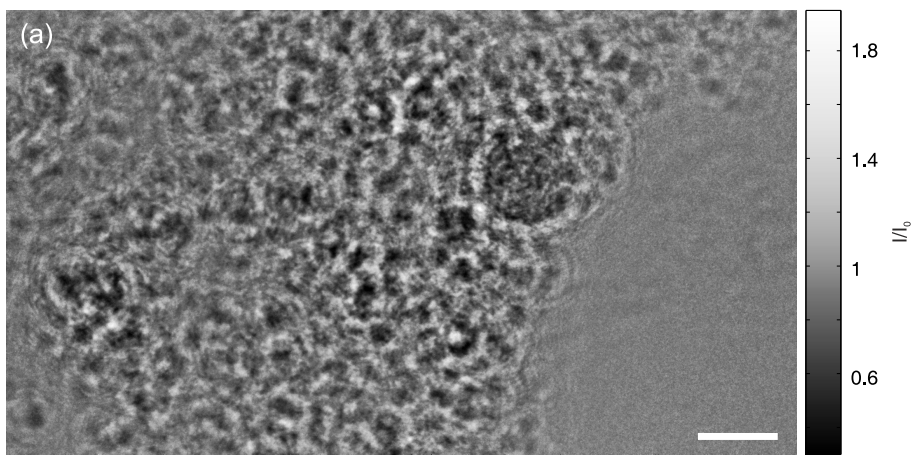

(b)

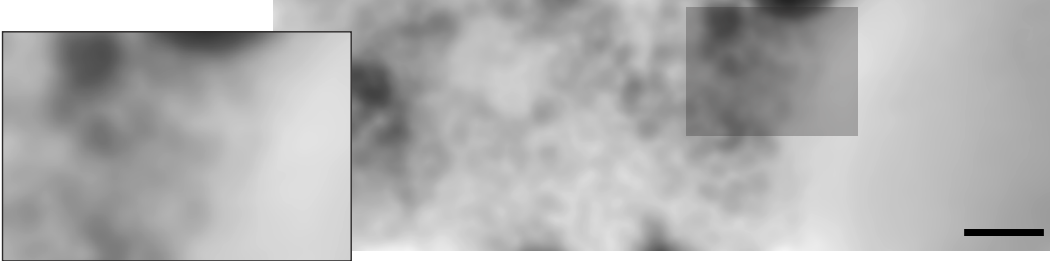

(c)
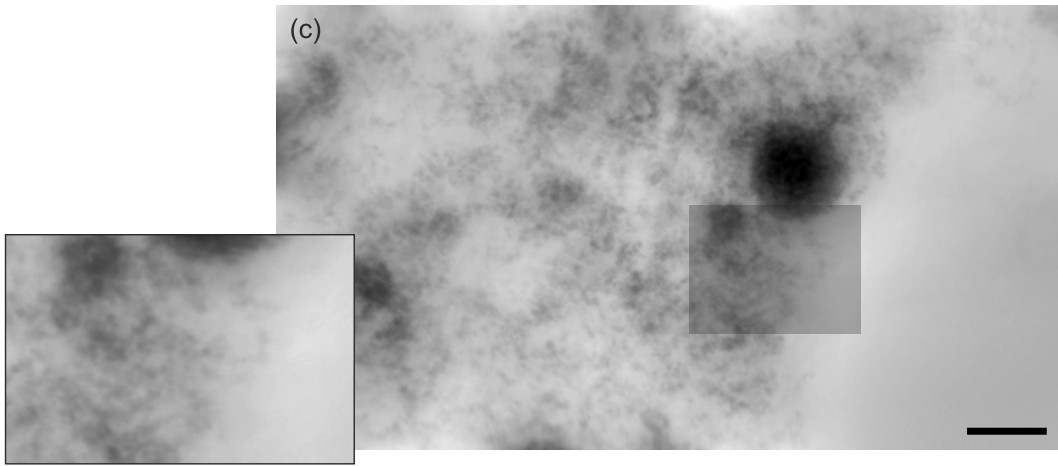

Figure 7.6: Typical projection data in the high-resolution setting. (a) Holographic phase-contrast image of the region marked by the dashed, red rectangle in Fig. 7.5(a), with (b) its corresponding SMO phase-retrieval and (c) the CTFbased phase-retrieval. The region marked by the dark rectangle is shown as a magnification in the inset. Scale bars denote $10 \mu \mathrm{m}$ 
If the sample is moved closer to the waveguide to obtain a larger magnification, the Fresnel number becomes even smaller (cf. Eq. (6.3)) and images appear more holographic. This results in a larger magnification and the region marked by the dashed rectangle in Fig. 7.5(a) is aligned in the beam. Figure 7.6 shows a representative projection of the same sample, recorded in the high-resolution setting. Already in the holograms, the structures from Fig. 7.5 can be clearly recognized, which now exhibit even more interference fringes. As before, MBA and CTF reconstructions are compared in Fig. 7.6(b) and (c), respectively, where the inset shows a magnification to compare the high resolution information. Low frequencies are regularized using $\alpha_{1}=5 \cdot 10^{-4}$ according to Eq. (2.45). To better compensate zeros in the $\mathrm{PCTF}$, the CTF-based phase-retrieval is performed using four distances and a smaller $\alpha_{2}=0.05$. Compared to the CTF reconstruction that contain high resolution information, the phase map retrieved with the MBA is significantly blurred. Interestingly, the phase map in Fig. 7.6(b) seems to have a higher resolution than that in Fig. 7.5(b), although for structures of the same size, no difference would be expected in the MBA reconstruction.

In general, no severe artifacts can be observed in the projections obtained after CTF-based phase-retrieval. As most beamlines operate mainly in the directcontrast regime, it is common practice to use the MBA or Paganin phase-retrieval $[164,180,113]$. However, reconstructions in this chapter show that, as long as the spectral bandwidth is not too large, the CTF based reconstruction can be used instead, which generally performs better at reconstructing high spatial frequencies and information about fine details in an image.

\subsection{Experimental results}

\subsubsection{Phase-contrast imaging of asthmatic mouse lungs}

Phase-contrast tomography measurements are performed for several samples of lung-slices from a total of five different mice. For this section, data is recorded in the large-FOV setting, i.e., measured images and phase retrieval will be similar to those shown in Fig. 7.4. The tomographic reconstruction of datasets shown in this section, is performed using the previously reconstructed CTF phase-maps as input for a filtered back projection (FBP) algorithm (PyHST, ESRF, Grenoble). Figure 7.7 shows two reconstructed orthoslices, obtained from a mouse with asthma (a) and from a control mouse (b). The gray scale is chosen so that dark regions correspond to a high electron density, where less dense structures become white. The 

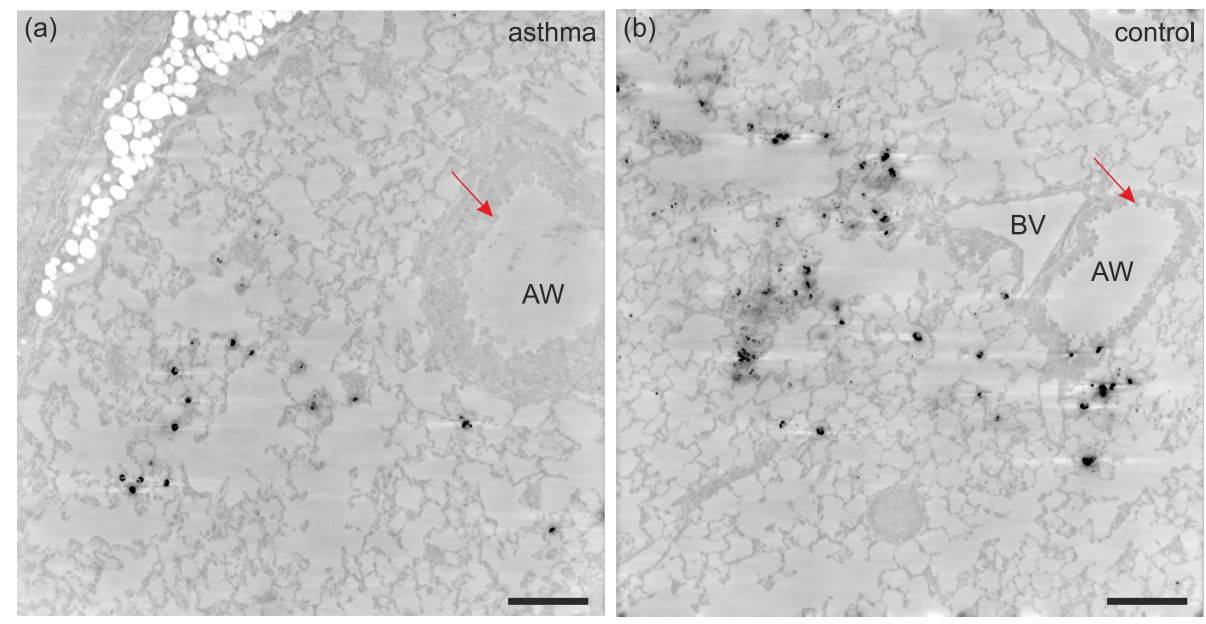

Figure 7.7: (a) Representative orthoslice showing the lung-structure of an asthmatic mouse in comparison to (b) a representative orthoslice of a control mouse. Darker values correspond to a higher electron density, so barium particles (black) and fat (white) can be easily distinguished from the soft tissue (dark gray) and water (light gray). Blood-vessels $(B V)$ and airways $(A W)$ can be identified based on their different wall morphologies. Note that the thickness of the cell layers around the airways (red arrow) is much thicker in asthmatic mice than in the controls. Scale bars denote $100 \mu \mathrm{m}$.

sample is surrounded by water ${ }^{1}$ which in this gray-value scaling appears as light gray. Additionally, barium sulfate (black), fat (white) and soft-tissue structures (darker gray) can be easily distinguished based on their different electron densities.

As barium sulfate was only present in the exogenous macrophages, i.e., the cells that do not belong to the organisms own immune system, every barium cluster can be identified as an injected macrophage. The soft-tissue structures correspond to structures known from histological microscope images (see Fig. 7.1) and thus blood vessels (BV) and airways (AW) can be distinguished, based on their wall morphology. The red arrows in Fig. 7.7 point to single goblet cells protruding into the airways, which are clearly resolved. Goblet cells are responsible for the mucus production in the bronchial tubes. The mucosa in the bronchial tubes cannot be resolved, as its electron density is too similar to that of water. However, especially in the marked bronchial tube of the asthmatic mouse (a), cells can be recognized that seem to be floating in the airway, which probably are attached to the mucosa.

1 Actually the sample is surrounded by PBS, which has nearly the same refractive index as water. 
Note that the cell layer around the airways is much thicker in asthmatic mice than in control mice, in correlation with histology results [43].

The great advantage of tomography is that virtual slices as in Fig. 7.7 can be obtained in arbitrary directions through the object and 3D information of the whole volume can be obtained, where in contrast to stacking histology, no slices have to be aligned and an isotropic voxel size is present. Figure 7.8(a) shows a $3 \mathrm{D}$ rendering of a dataset of an asthmatic mouse. Three orthoslices are shown, together with several renderings of relevant structures in the lung. All macrophages are segmented based on their density by thresholding the histogram and are displayed as green dots. ${ }^{2}$ A blood vessel (purple) is semi-manually labeled using the contrast-dependent blow-tool, implemented in Avizo. The intricate 3D structure of soft-tissue, e.g., the alveolar walls and other unlabeled cells, are automatically labeled with the magic-wand tool of Avizo, followed by the application of a watershed algorithm [20]. The resulting segmentation is limited to a nonrectangular ROI by binary multiplication and rendered in half-transparent yellow. Figure 7.8(b) shows an orthoslice perpendicular to the tomographic rotation axis. As is indicated by the arrows, some cells seem to be surrounded by soft tissue. The yellow ROI, which is displayed in the 3D rendering, corresponds to the region of those cells. The thick, purple blood vessel divides into many branches and one of them ends in this particular ROI, to enable oxygen exchange between the breathed air and the blood. Figure 7.8(c) shows a close up of the ROI from a different viewing angle, which indicates that many macrophages seem to be surrounded by soft tissue also in 3D. Hence, the hypothesis can be formed that the exogenous macrophages might be able to migrate from the lumen through the epithelial cells. However, to investigate the precise location, a higher resolution is necessary. As the full information contained in the 3D dataset can be barely appreciated from 2D snapshots, a video of the 3D rendering ${ }^{3}$ shown in Fig. 7.8 is available as supplementary online material in [89].

Figure 7.9 shows 3D renderings of datasets obtained from several samples of asthmatic and control mice. Two further datasets were also successfully reconstructed that are not shown here. Instead, we refer the reader to the publication of the present experiments [89], where these are shown in the supplementary information. The mouse number $(\mathrm{Mxxx})$, the ascending number of the specific lung slice sample (\#x) as well as the type of the mouse (blank, control or asthma) are denoted next

${ }_{2}$ More precisely the barium-sulfate particles are segmented. But as barium sulfate was only present in the exogenous macrophages, it can be identified as those cells.

3 Link to the video: http://www.nature.com/srep/2015/150512/srep09973/extref/srep09973s1.avi 

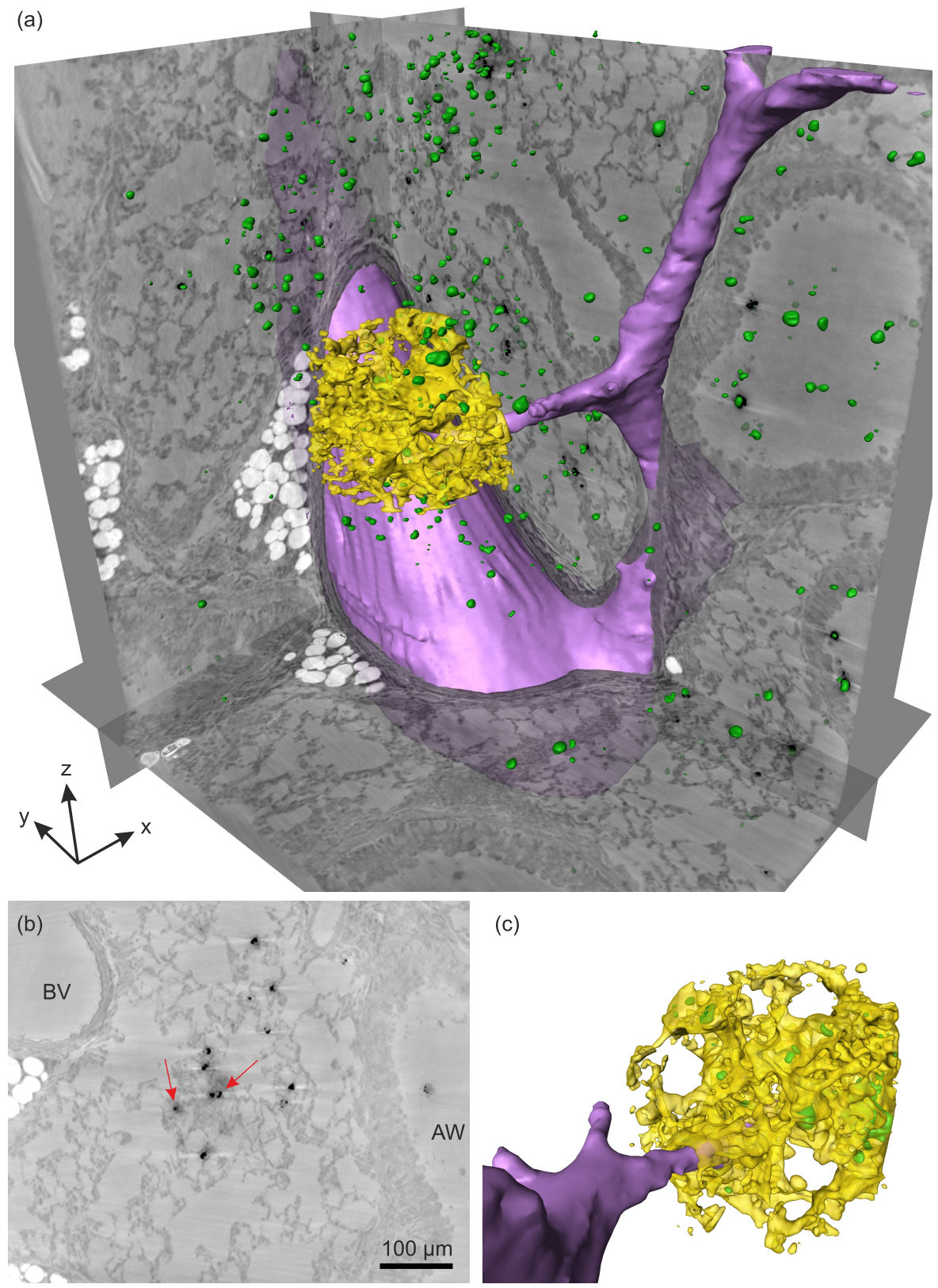

(c)

Figure 7.8: $3 D$ reconstruction of data as shown in Fig. 7.4. (a) $3 D$ rendering showing three orthoslices together with barium particles (green), soft-tissue structures in a ROI (yellow) and a blood-vessel (purple). (b) xy-orthoslice of the same volume, showing single macrophages surrounded by soft tissue (red arrows). (c) shows a close up of the ROI under a different viewing angle. 

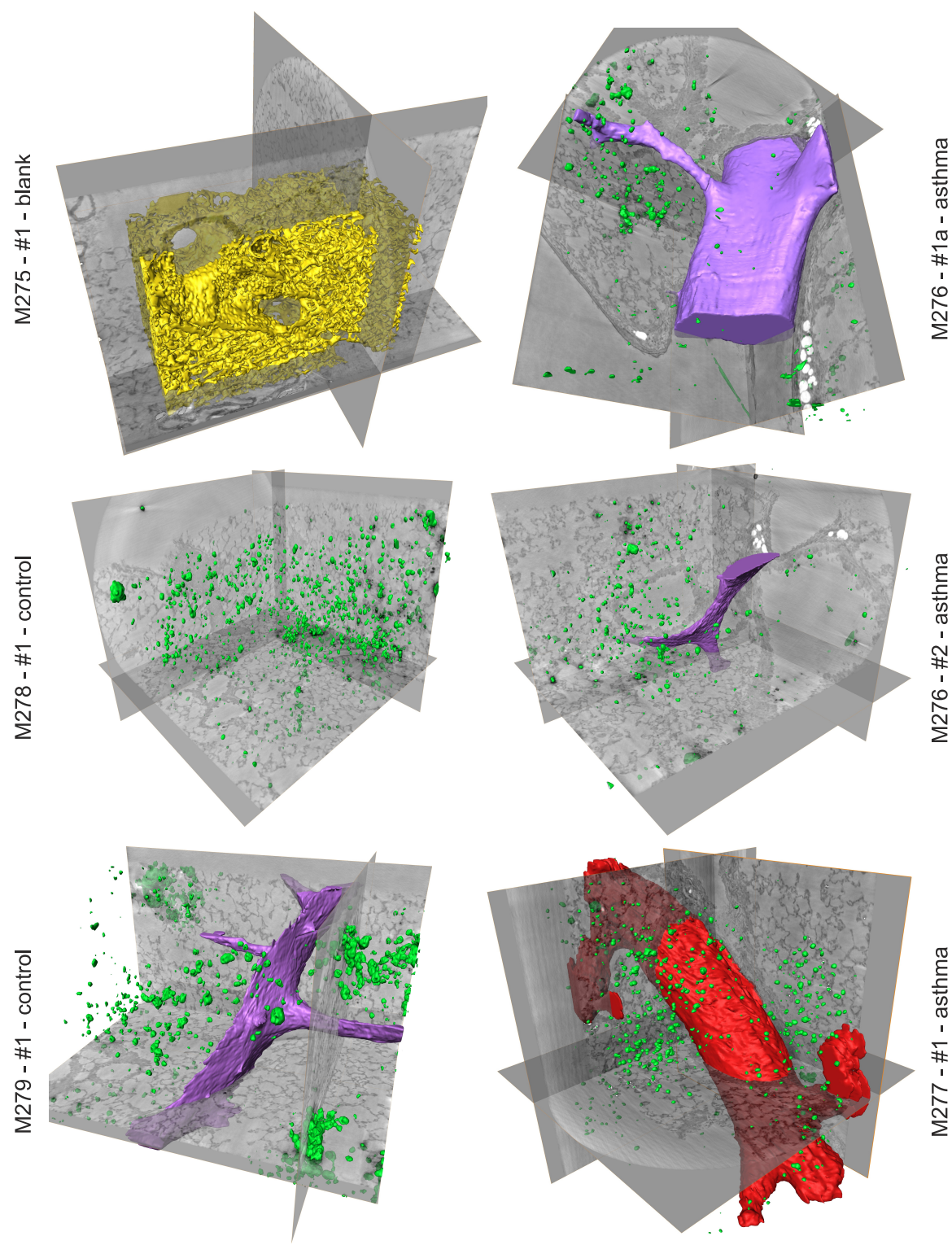

Figure 7.9: Overview of datasets recorded at the ID22 beamline. Shown are $3 D$ renderings recorded from a total of five different mice. One blank without labeled macrophages (top left), two control mice without asthma (left) and reconstructions from three different samples of two asthmatic mice (right). The rendering in Fig 7.8 stems from a different region of the same sample for which the reconstruction is shown at the top right. The color code is as before, where soft-tissue is rendered in yellow, blood vessels are shown in purple and barium particles in green. The inner region of a bronchial tube is shown in red for one dataset. 


\begin{tabular}{|c|c|c|c|c|}
\hline & blank & asthma & control & shown in \\
\hline M275 & $x$ & & & Fig. 7.9 \\
\hline M276 \#1a & & $x$ & & Fig. 7.9 \\
\hline M276 \#1b & & $x$ & & - \\
\hline M276 \#1c & & $x$ & & Fig. 7.8 \\
\hline M276 \#1d & & $x$ & & - \\
\hline M276 \#2 & & $x$ & & Fig. 7.9 \\
\hline M277 & & $x$ & & Fig. 7.9 \\
\hline M278 & & & $x$ & Fig. 7.9 \\
\hline M279 & & & $x$ & Fig. 7.9 \\
\hline
\end{tabular}

Table 7.2: Overview of different samples measured in the large-FOV setting.

to the renderings. The rendering shown in Fig. 7.8 stems from the mouse M276 sample \#1, where a different region inside the lung slice was chosen. Table 7.2 gives an overview of all the datasets. By visual inspection, bronchial walls are found to be thicker in asthmatic mice, as can be seen in the representative slices in Fig. 7.7, but the location of the macrophages does not seem to differ. Note that for the $3 \mathrm{D}$ renderings, the datasets are resampled by a factor of two.

\subsubsection{Zoom tomography enabled by x-ray waveguides}

With the goal of obtaining reconstructions at a higher resolution, the waveguide based setup at the GINIX instrument is used to image lung-tissue samples. Figure 7.10 shows reconstructed orthoslices in the medium-FOV (b) and in the highresolution setting (c), of datasets for which measured projections and CTF-based phase-retrievals are shown in Fig. 7.5 and 7.6, respectively. Relevant parameters are listed in Tab. 7.1. The investigated sample shows the lung structure of a control mouse, with intratracheally instilled barium-labeled macrophages. As in the large-FOV setting, barium particles can be clearly identified based on their electron density. A blood vessel and two airways can be recognized in Fig. 7.10(a). A part of the bronchial wall is manually labeled, for which the dashed, orange line shows the outline of the segmentation. The region marked by the red, dashed rectangle in Fig. 7.10(a) denotes the ROI, used for a second measurement, where the resulting orthoslice in Fig. 7.10(b) shows the same structures at a higher resolution. Single macrophages with a typical diameter of 10 to $15 \mu \mathrm{m}$ can be identified based on the positions of barium-sulfate particles. To this end, the outline of a single cell is marked by a dashed, blue line in Fig. 7.10(b).

Figure 7.11(a) shows a $3 \mathrm{D}$ rendering of the dataset recorded in medium-FOV 

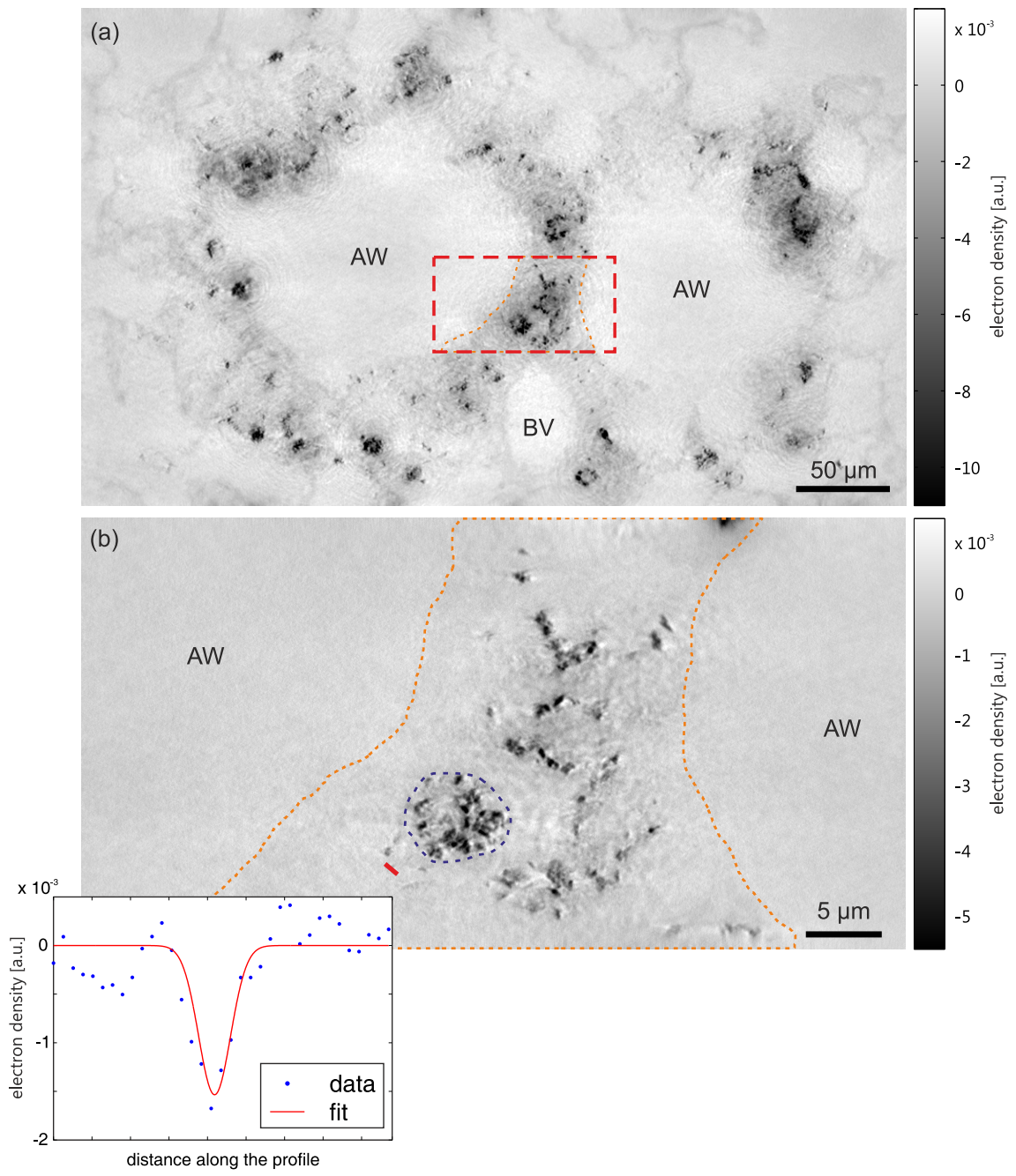

Figure 7.10: (a) Reconstructed orthoslices of the dataset recorded in the medium-FOV setting, in which airways $(A W)$ and a blood vessel $(B V)$ can be identified. The dashed, red rectangle denotes the ROI used for the highresolution scan. (b) Orthoslice obtained in the high-resolution setting, where the region used for manual segmentation of the bronchial wall is marked by the dashed, orange line. A dashed line indicates the outline of a single cell which is manually segmented and displayed in the following $3 D$ renderings. A profile through the red solid line is plotted in the inset, revealing a feature size of 249 nm FWHM. 
setting. As before, barium particles are automatically segmented based on their reconstructed electron density and displayed in green. A blood vessel is semimanually segmented and displayed in purple. A bronchial tube (not rendered) that splits up into two branches lies parallel to this blood vessel. A part of the bronchial wall is manually segmented and displayed in half-transparent yellow. Figure 7.11(b) shows the rendering from a different viewing angle, where additionally in one of the orthoslices the splitting bronchial tube can be seen. The rendering of barium particles in Fig. 7.11(a) is limited to about half-the volume, whereas Fig. 7.11(b) shows all barium-particles that are partly hidden behind the half-transparent orthoslices. The ROI at the bronchial wall is displayed as an isolated close-up in Fig. 7.11(c), in which the manually segmented single macrophage is shown in blue. It can be clearly seen that macrophages are located inside the bronchial wall. A video of the $3 \mathrm{D}$ rendering ${ }^{4}$ of Fig. 7.11 can be found in the supplementary information of [89].

Figure 7.12 shows a 3D rendering of the dataset measured in the high-resolution setting, which was obtained by decreasing the distance between same and waveguide to increase the geometric magnification. Four distances leading to different Fresnel numbers are used for the CTF-based phase-retrieval, where all measured projections are rescaled and aligned to each other, using single-distance CTF reconstructions (see section 6.3.1.2). To correct for temporal instabilities of the illumination, several empty-beam images are recorded by moving the sample out of the beam during the measurement. As the motors do not move perfectly, projections directly before and after the recordings of new empty images are registered, which significantly improves the 3D reconstruction. For the 3D rendering in Fig. 7.12, barium-sulfate particles are again segmented based on their density and rendered in green. The same bronchial wall segmentation as in Fig. 7.11 is displayed in half-transparent yellow to facilitate orientation. A single-cell is manually labeled and its outline is shown in half-transparent blue inside the bronchial wall in (a), as well as in isolated form from different viewing angles in (b). The orthoslice in Fig. 7.10(b) shows a cut through this particular cell.

The higher resolution of the dataset in Fig. 7.12 immediately becomes apparent upon inspection of barium particles. Due to the high resolution, not only the location of macrophages within the bronchial wall can be confirmed, but the internal barium-sulfate distribution in single cells is also accessible. The barium-sulfate particles are not homogeneously distributed inside the cells, but seem to mainly

${ }_{4}^{4}$ Link to the video: http://www.nature.com/srep/2015/150512/srep09973/extref/srep09973s2.avi 
(a)
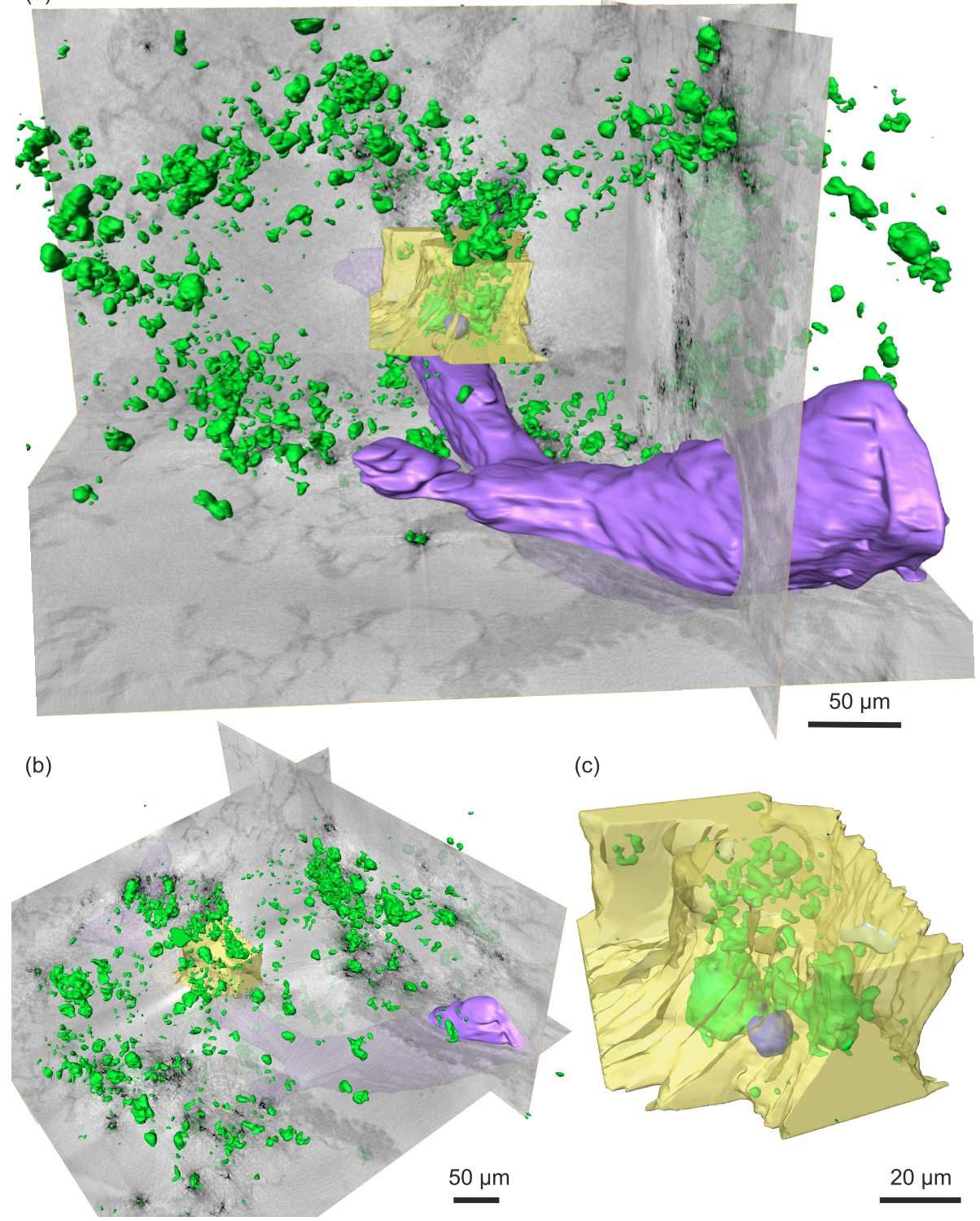

(c)

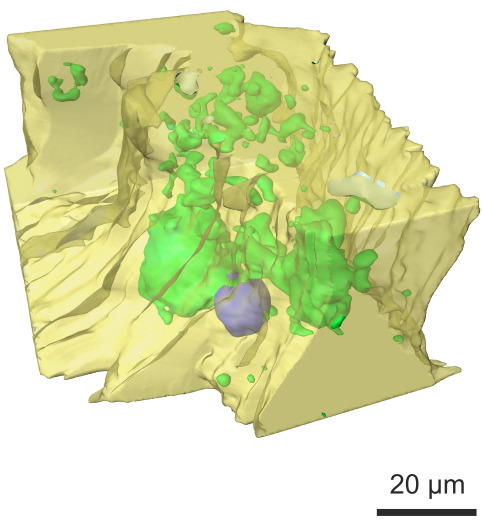

Figure 7.11: Results obtained in medium-FOV setting using a waveguide illumination. (a) 3D Rendering showing barium particles (green), a blood vessel (purple) and part of a bronchial wall (yellow) inside a ROI. The outline of a single macrophage inside this ROI is rendered in blue. The inset shows an orthoslice of the ROI area with the region used to segment the bronchial wall (dashed orange line). (b) shows a rendering with orthoslices at different positions from another viewing angle. (c) Close-up of the ROI that corresponds to the region used in the high-resolution setting. 
(a)

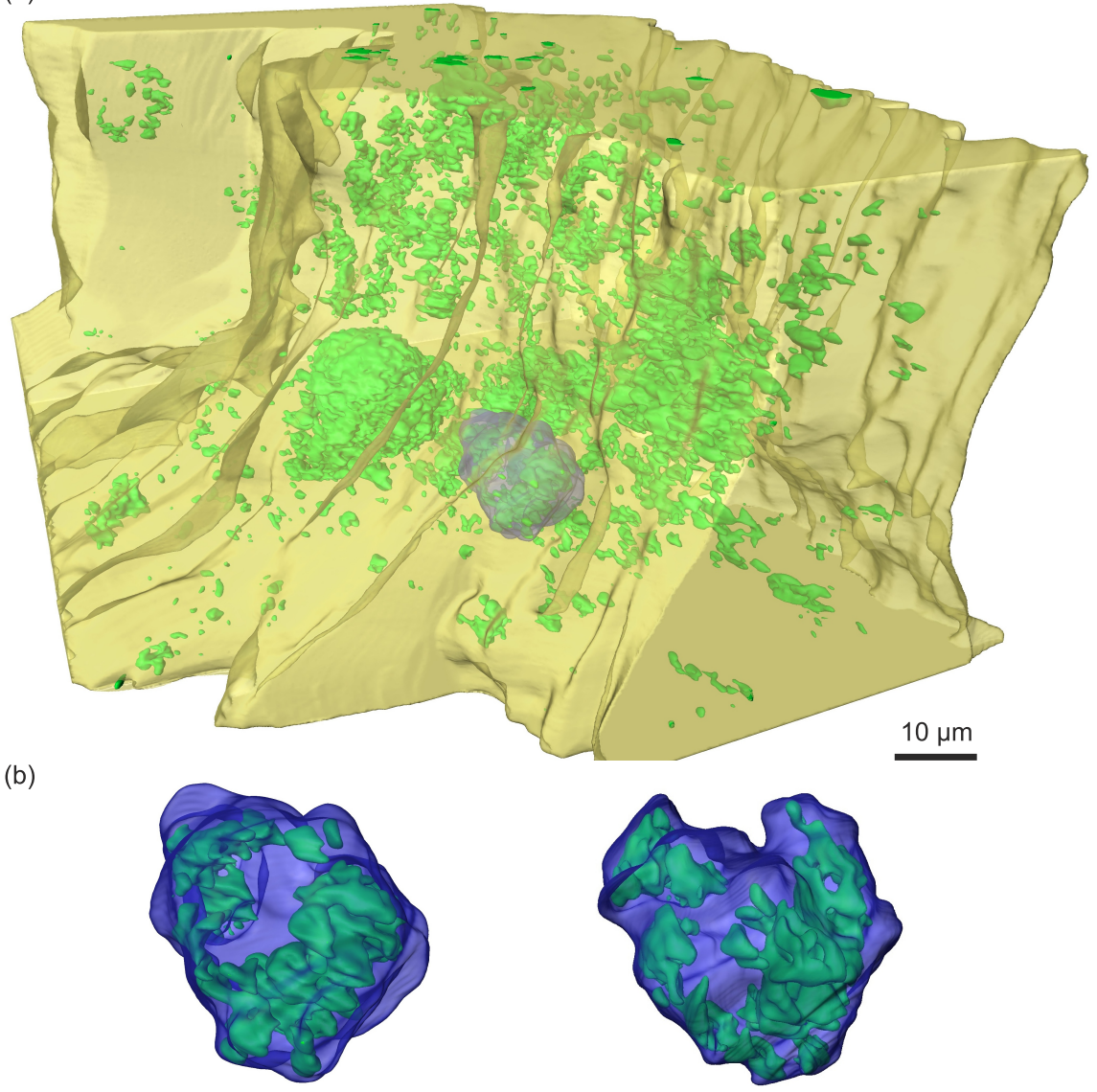

Figure 7.12: 3D rendering of the high-resolution volume. (a) shows the segmented bronchial wall structure (half-transparent yellow), with barium particles (green) and the outline of a single cell (half-transparent blue). (b) An isolated close-up of the segmented cell from two different viewing angles. 
locate at the edges of macrophages (close to the cell membrane), which is in good agreement with the results obtained in the previous chapter for macrophages in aqueous surroundings and embedded in resin (see Fig. 6.14 and 6.21). However, as contrast from particular cell-bodies is not achieved in this high-resolution measurement, the outlines of single macrophages can only be estimated based on the distribution of barium particles. Especially in regions of high barium concentration, the discrimination of single cells is hampered. To obtain a better impression of the $3 \mathrm{D}$ volume, a video of the rendering ${ }^{5}$ shown in Fig. 7.12 is available in the supplementary information of [89].

\subsubsection{Dose and resolution}

Compared to the measurements at the ID22 beamline, the resolution could be significantly increased by using a waveguide illumination at the GINIX instrument. While the resampled pixel size limits the resolution in the ID22 reconstructions, residual drifts seem to be limiting the high resolution reconstructions in the waveguide experiments. One way to overcome such limitations would be the application of motion correction algorithms as presented before. However, as the reconstructions are obtained from interior-tomography measurements, structures outside the FOV would heavily disturb the alignment algorithms. Figure 7.10 shows a profile of the reconstructed values through a small feature inside the bronchial wall. An error function fit to this profile reveals a FWHM of $249 \mathrm{~nm}$, posing an upper limit to the resolution. To better quantify the 3D resolution, the Fourier shell correlation (FSC) is employed. To this end, two independent datasets are reconstructed by taking every second angle into account. The correlation of the datasets is subsequently calculated by means of Eq. (1.94) and compared to the 1/2-bit threshold. The resulting FSC and threshold curves are presented in Fig. 7.13(a) and (b) for

\begin{tabular}{llll}
\hline & large-FOV & medium-FOV & high-resolution \\
\hline total fluence [photons $/ \mathrm{um}^{2}$ ] & $1.9 \cdot 10^{8}$ & $6.9 \cdot 10^{6}$ & $2.1 \cdot 10^{8}$ \\
dose $[\mathrm{Gy}]$ & $5.6 \cdot 10^{4}$ & $3.0 \cdot 10^{3}$ & $9.1 \cdot 10^{4}$ \\
3D resolution $[\mathrm{nm}]$ & 860 & 651 & 170 \\
\hline
\end{tabular}

Table 7.3: Fluence and dose values for the different experimental settings. The $3 D$ (half-period) resolution for the large-FOV dataset is determined by the resampled pixel-size. For the medium-FOV and high-resolution setting, the resolution is determined with the FSC.

${ }^{5}$ Link to the video: http://www.nature.com/srep/2015/150512/srep09973/extref/srep09973s3.avi 

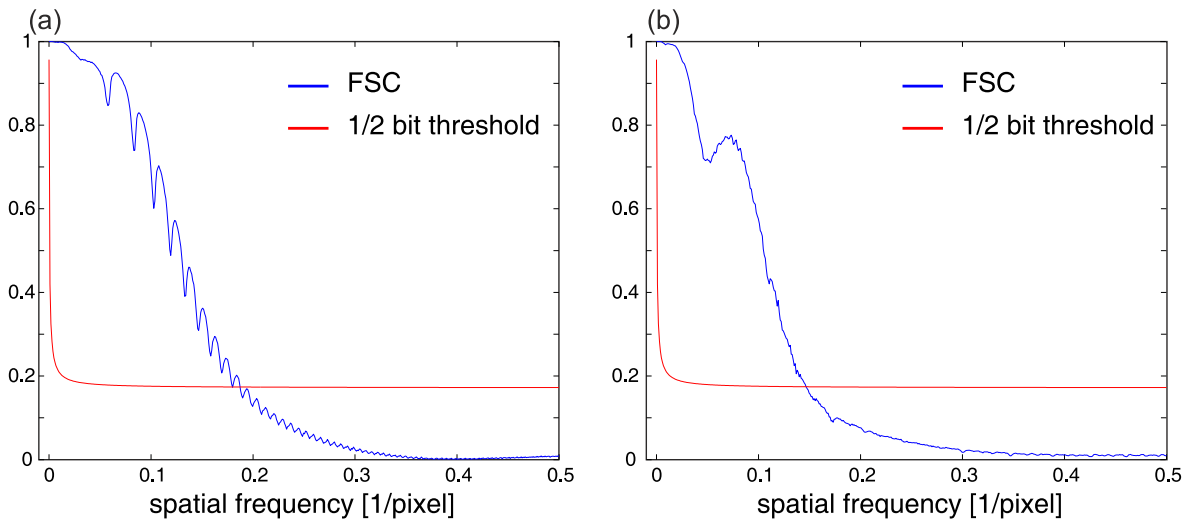

Figure 7.13: Fourier shell correlations of the waveguide reconstructions for (a) the medium-FOV setting and for (b) the high-resolution setting that was used to determine the (half-period) resolution of the datasets, listed in Tab. 7.3.

the reconstructions of Fig. 7.11 and 7.12, respectively. The intersections of the FSCs with the threshold curves determine the maximum spatial frequency that contains sufficient information for interpretation [176]. Corresponding half-period resolution-values are listed in Tab. 7.3. Some oscillations can be observed in the FSC for the medium-FOV reconstruction (Fig. 7.13(a)). These are caused by zeros in the PCTF as only a single distance was used for the CTF-based phase-retrieval in this case.

Table 7.3 shows fluence and dose values (Eq. (1.92)) for the different experimental setups. Note that in particular the dose present at the ID22 beamline is more than 18-times higher, although a slightly lower resolution is achieved. The flux-density, i.e., the number of incident photons per second, was significantly higher at the ID22 beamline. Hence, it was not possible to obtain high-resolution reconstructions by choosing a larger geometric magnification at the ID22 beamline, as air-bubbles started to form in the liquid environment during the measurement. Note that also in ptychographic x-ray tomography, the necessary dose to obtain reconstructions at a similar resolution was about 20-times higher [41].

\subsection{Summary}

In this chapter we have demonstrated the application of propagation-based phasecontrast tomography to lung-tissue slices of asthmatic and control mice. Enabled 
by cone-beam geometry of the x-ray illumination, the measurement of large fields of view covering several $100 \mu \mathrm{m}$ is possible, where a high resolution down to about $170 \mathrm{~nm}$ can be achieved for selected interior regions of interest of the same sample. One goal of the experiments was to keep the samples as close as possible to in situ conditions, which is enabled by a special liquid chamber. In addition to the barium-stained macrophages, the unlabeled, internal 3D structure of the mouse lungs could be reconstructed, which shows a very high correspondence to structures known from conventional histology. By using a waveguide-based illumination, only the coherent part of the $\mathrm{x}$-rays is transmitted and in particular due to the strongly improved illumination, artifact-free imaging in the deep-holographic regime now becomes feasible. This combination allows dose-efficient reconstructions and we expect that the resolution could be further increased, by developing new alignment algorithms or improving the mechanical stability.

Equipped with this methodology, important biomedical questions can now be addressed. In particular, we could reconstruct the precise locations of intratracheally injected macrophages in relation to anatomical structures. The 3D reconstructions revealed that one day after injection, macrophages are located within the alveolar lumen and within the bronchial walls. Thus, it seems that the exogenously applied MH-S macrophages are able to migrate from the airway lumen through the epithelium. 


\section{Conclusions}

The main goal of this thesis was to extend the method of propagation-based phasecontrast tomography to allow 3D imaging at sub-cellular resolution in whole organs. To this end, three classes of experiments were carried out on different samples. The first part was dedicated to the application of in-line holography for imaging of whole mouse lungs. To maintain in situ conditions, a special preparation of fresh mice was used and immediate tomographic measurements in an optimized experimental configuration could reveal the overall anatomy of the mouse chest. Using the zoom-capabilities of a cone-beam geometry, phase contrast inside the lungs of the mice could be observed at large magnifications. With suitable phase-retrieval algorithms for the direct-contrast regime, 3D volumes with unprecedented data quality could be reconstructed, which allow the automatic 3D rendering of airways and alveolae at a resolution of about $5 \mu \mathrm{m}$. One key point to avoid motion artifacts was to filter the energy spectrum. Further developments of new x-ray sources will enable the measurement of comparable results in a few minutes instead of hours. As no synchrotron radiation is needed, the use of suitable automated analysis algorithms will enable biological studies with many samples, which are needed to obtain statistically significant results.

The second branch of experiments had the goal to investigate the high-resolution capabilities of propagation-based phase-contrast imaging using single cells as specimens. A variety of phase-retrieval algorithms was implemented and successfully applied to reconstruct holograms from dried macrophages. A newly developed phase-retrieval algorithm based on the transport of intensity equations, which does not pose restrictive assumptions to the object, showed the best performance at high-resolutions down to $50 \mathrm{~nm}$. The influence of different contrast agents was analyzed and high resolution projections of stained and unstained cells could be obtained. The native internal structure of single cells could be identified by means of an osmium tetroxide stain that, however, is not compatible with in vivo experiments. As an alternative contrast agent, barium sulfate is used to stain single cells and the precise location of barium particles inside macrophages has been revealed in automatically segmented 3D renderings. In particular the application of motion correction algorithms and combined 3D phase-retrieval approaches enable 
a very high $3 \mathrm{D}$ resolution below $50 \mathrm{~nm}$. A prerequisite to achieve such results was the use of optimized x-ray waveguide channels that provide a very clean and controlled illumination. Compared to recent ptychographic reconstructions [184], the invested radiation dose is significantly lower. This dose efficiency allows measurements of samples in aqueous conditions, which was demonstrated by providing $2 \mathrm{D}$ reconstructions of living cells. Another main benefit of in-line holography is that even very simple phase-retrieval algorithms like the CTF-based approach already deliver quite good first impressions of the object, which can be used as a direct feedback during an experiment at a synchrotron facility.

The aim of the last experiments was to obtain high-resolution 3D reconstructions of large pieces of murine lung-tissue. Using barium-sulfate as a non-invasive contrast agent, the precise location of macrophages in thick lung-tissue slices is obtained by phase-contrast tomography measurements. The experiments were carried out with samples from asthmatic and control mice that showed a typical thickening of bronchial walls in the asthma case but no significant differences in the distribution of macrophages. The waveguide illumination enables measurements in a conebeam geometry with a flexible choice of resolution and field of view. By this means, we could successfully reconstruct a large field of view with several $100 \mu \mathrm{m}$ side length and for the same sample a high resolution dataset with $170 \mathrm{~nm} 3 \mathrm{D}$ resolution, without significant changes in the sample.

A key point to obtain high resolution 3D reconstructions is the fact that propagation imaging provides a full-field imaging technique. In contrast to ptychography, no lateral scanning is necessary for the reconstruction so that data is recorded during a smooth rotation without translational drifts. Thus, the reconstruction quality does not depend on successful application of motion correction algorithms, which do not properly work in the case of interior tomography. Nevertheless, if combined with moderate scanning, the measurement of a whole organ like the lung should be easily possible without any loss in resolution. In the present results, macrophages in the lung can be clearly identified with sub-cellular resolution, based on the distribution of barium particles. They were found in alveolae and between the soft tissue of bronchial walls, indicating that the cells might be able to migrate through epithelial cell layers. Future experiments will target to include multiple cell makers, so that specific cell tracking will enable functional imaging using phase-contrast tomography. Additionally, if a relaxed resolution of about 5 $\mu \mathrm{m}$ is sufficient to track the cells, the approach can be translated to laboratory experiments and thus the availability of the method will be increased strongly.

In conclusion, holographic x-ray imaging was successfully applied to biomedical 
samples revealing features over a wide range of resolutions and fields of view for whole organs like the mouse lung down to sub-cellular resolution in thick tissue slices and isolated cells embedded in different environments. 



\section{Appendix}

\section{A.1 The Fourier transform and its properties}

In the literature, different forms of the Fourier transform can be found. As also two of the most relevant references for this thesis, namely the "Introduction to Fourier optics" by Goodman [55] and "Coherent X-Ray Optics" by Paganin [133] use different definitions of the Fourier transform, we will clarify the definitions, followed by some general properties of the Fourier transform.

The definition of the Fourier transform $\mathcal{F}$ of a scalar function $f(x)$ used in [133] is

$$
\mathcal{F}(f(x)):=\tilde{f}\left(k_{x}\right)=\frac{1}{\sqrt{2 \pi}} \int_{-\infty}^{\infty} f(x) \mathrm{e}^{-\mathrm{i} k_{x} x} \mathrm{~d} x .
$$

Thus the inverse Fourier transform $\mathcal{F}^{-1}$ is defined by

$$
\mathcal{F}^{-1}\left(\tilde{f}\left(k_{x}\right)\right):=f(x)=\frac{1}{\sqrt{2 \pi}} \int_{-\infty}^{\infty} \tilde{f}\left(k_{x}\right) \mathrm{e}^{\mathrm{i} k_{x} x} \mathrm{~d} k_{x}
$$

For functions depending on vector quantities $f(\mathbf{r})$ the Fourier transform can be generalized such that for each component of $\mathbf{r}$ a scalar Fourier transform is performed individually

$$
\mathcal{F}(f(\mathbf{r})):=\tilde{f}(\mathbf{k})=\frac{1}{(2 \pi)^{3 / 2}} \int_{\mathbb{R}^{3}} f(\mathbf{r}) \mathrm{e}^{-\mathrm{i} \mathbf{k} \cdot \mathbf{r}} \mathrm{d} \mathbf{r},
$$

with the back transform analogous to Eq. (A.1).

The Fourier transform for spatial frequencies, as used in [55] is

$$
\mathcal{F}(f(x)):=\tilde{f}\left(\nu_{x}\right)=\int_{-\infty}^{\infty} f(x) \mathrm{e}^{-2 \pi \mathrm{i} \nu_{x} x} \mathrm{~d} x
$$


Thus the inverse Fourier transform $\mathcal{F}^{-1}$ is defined by

$$
\mathcal{F}^{-1}\left(\tilde{f}\left(\nu_{x}\right)\right):=f(x)=\int_{-\infty}^{\infty} \tilde{f}\left(k_{x}\right) \mathrm{e}^{2 \pi \mathrm{i} \nu_{x} x} \mathrm{~d} \nu_{x},
$$

which is generalized to higher dimensions as before, without any prefactors. In this thesis, the explicit dependence either on $\mathbf{k}_{\perp}$ or on the spatial frequencies $\boldsymbol{\nu}_{\perp}=\mathbf{k}_{\perp} / 2 \pi$ determines, which Fourier transform has to be used.

Without any proof, we note that the Fourier transform is linear, i.e.

$$
\mathcal{F}(a f(x)+b g(x))=a \mathcal{F}(f)(k)+b \mathcal{F}(g)(k),
$$

and the convolution theorem holds

$$
\mathcal{F}(f(x) * g(x))=\mathcal{F}(f(x)) \cdot \mathcal{F}(g(x)) .
$$

The Fourier transform of the Dirac-delta-distribution can be easily calculated to

$$
\mathcal{F}_{\perp}\left(\delta\left(\mathbf{r}_{\perp}\right)\right)=2 \pi \delta_{D}\left(\mathbf{k}_{\perp}\right)=\delta_{D}\left(\boldsymbol{\nu}_{\perp}\right)
$$

\section{A.2 Frechét derivative of the propagation operator}

The Frechét derivative $F^{\prime}$ of an operator $F: D \rightarrow Y, \psi \mapsto F(\psi)$, with $D \subset X$ and $X, Y$ being some Hilbert spaces, is defined via

$$
\lim _{\|h\| \rightarrow 0} \frac{1}{\|h\|}\left\|F(\psi+h)-F(\psi)-F^{\prime}(\psi) h\right\|=0 .
$$

One way to describe Fresnel propagation it given by Eq. (1.32), hence the operator $F$ can be written as

$$
F(\psi) \propto\left|\int_{\mathbb{R}^{2}} \psi(\mathbf{r}) \mathrm{e}^{\mathrm{i} \chi\left(\mathbf{r}_{0}\right)} \mathrm{e}^{-\mathrm{i} \boldsymbol{\xi} \cdot \mathbf{r}_{0}} \mathrm{~d} \mathbf{r}_{0}\right|^{2},
$$

with $\boldsymbol{\xi}=\frac{\mathrm{i} k}{z} \mathbf{r}_{1}$ and $\chi\left(\mathbf{r}_{0}\right)=\frac{\mathrm{i} k}{2 z} \mathbf{r}_{0}^{2}$. We write the wave function as a general complex number

$$
\psi=\mathrm{e}^{\mathrm{i} \phi-\mu / 2},
$$


with phase $\phi$ and absorption $\mu$. Thus the small change $h$ can be written as a small change in amplitude and phase. For the sake of simplicity, we limit the considerations to a pure phase object, which acts as a constraint to the IRGN method

$$
\psi+h=\mathrm{e}^{\mathrm{i}(\phi+h)}=\psi \cdot\left(1+\mathrm{i} h+\mathcal{O}\left(h^{2}\right)\right) .
$$

Substituting this expression in $F(\psi)$ yields

$$
\begin{aligned}
F(\psi+h) \propto & \left|\int_{\mathbb{R}^{2}} \psi(\mathbf{r})\left(1+\mathrm{i} h+\mathcal{O}\left(h^{2}\right)\right) \mathrm{e}^{\mathrm{i} \chi\left(\mathbf{r}_{0}\right)} \mathrm{e}^{-\mathrm{i} \boldsymbol{\xi} \cdot \mathbf{r}_{0}} \mathrm{~d} \mathbf{r}_{0}\right|^{2} \\
= & \left|\left(\int_{\mathbb{R}^{2}} \psi(\mathbf{r}) \mathrm{e}^{\mathrm{i} \chi\left(\mathbf{r}_{0}\right)} \mathrm{e}^{-\mathrm{i} \boldsymbol{\xi} \cdot \mathbf{r}_{0}} \mathrm{~d} \mathbf{r}_{0}\right)+\left(\int_{\mathbb{R}^{2}} \mathrm{i} h \psi(\mathbf{r}) \mathrm{e}^{\mathrm{i} \chi\left(\mathbf{r}_{0}\right)} \mathrm{e}^{-\mathrm{i} \boldsymbol{\xi} \cdot \mathbf{r}_{0}} \mathrm{~d} \mathbf{r}_{0}\right)+\mathcal{O}\left(h^{2}\right)\right|^{2} \\
= & F(\psi)+\mathcal{O}\left(h^{2}\right) \\
& +2 \cdot \operatorname{Re}\left[\left(\int_{\mathbb{R}^{2}} \mathrm{i} h \psi(\mathbf{r}) \mathrm{e}^{\mathrm{i} \chi\left(\mathbf{r}_{0}\right)} \mathrm{e}^{-\mathrm{i} \boldsymbol{\xi} \cdot \mathbf{r}_{0}} \mathrm{~d} \mathbf{r}_{0}\right) \cdot\left(\int_{\mathbb{R}^{2}} \psi(\mathbf{r}) \mathrm{e}^{\mathrm{i} \chi\left(\mathbf{r}_{0}\right)} \mathrm{e}^{-\mathrm{i} \boldsymbol{\xi} \cdot \mathbf{r}_{0}} \mathrm{~d} \mathbf{r}_{0}\right)^{*}\right],
\end{aligned}
$$

where for the last step the relation $z+z^{*}=2 \cdot \operatorname{Re}(z)$ for $z \in \mathbb{C}$ was used. Thus, with Eq. (A.9) it is

$$
F^{\prime}(\psi) h=2 \cdot \operatorname{Re}\left[\left(\int_{\mathbb{R}^{2}} \mathrm{i} h \psi(\mathbf{r}) \mathrm{e}^{\mathrm{i} \chi\left(\mathbf{r}_{0}\right)} \mathrm{e}^{-\mathrm{i} \boldsymbol{\xi} \cdot \mathbf{r}_{0}} \mathrm{~d} \mathbf{r}_{0}\right) \cdot\left(\int_{\mathbb{R}^{2}} \psi(\mathbf{r}) \mathrm{e}^{\mathrm{i} \chi\left(\mathbf{r}_{0}\right)} \mathrm{e}^{-\mathrm{i} \boldsymbol{\xi} \cdot \mathbf{r}_{0}} \mathrm{~d} \mathbf{r}_{0}\right)^{*}\right] .
$$

\section{A.3 List of Matlab functions}

In this section some function names are listed as they appear in the propagation imaging toolbox together with a short description and hints for their application. Some of the functions are actually wrapper functions, which call a more general version of it to allow the handling of astigmatism, i.e. different Fresnel numbers and/or magnifications for the horizontal and vertical direction. These general functions have the same functionality and are indicated with an _astig suffix, but are not separately shown in this list.

All functions requiring a structure called settings can be called without arguments to return a structure of default parameters, from which the name of all optional settings can be obtained. 


\section{A.3.1 Image preprocessing}

rescale_defocus_series (images, fresnelnumbers, magnifications, settings)

Resized and aligns images to each other depending on the magnifications given. All images are magnified to fit the pixel size of the first image given. Images have to be sorted such that the image with the largest magnification is the first. Fresnelnumbers are scaled according to the resized pixel size.

align_image (image, reference, settings)

Registers the image with respect to the given reference. This function is used by rescale_defocus_series, so the some settings are the same. The most important ones are the roi parameters (roil, roit, roir, roib), the highpass_range and lowpass_range to filter the images prior to registration. remove_outliers (image, [threshold=2], [filterrange=5])

Removes single pixel outliers in an image. The image is smoothed with a $2 \mathrm{D}$ median filter using the filterrange parameter. The standard deviation $\sigma$ of the difference between the real and the filtered image is calculated. All pixels that deviate more than threshold $\times \sigma$ are replaced by its median filtered value.

remove_profile (image, ranges, [filterpar=200])

Removes residual low-frequency profiles in the image by averaging image values in the given ranges. The ranges have to be given as a cell containing 2 or 4 entries, which denote the area over which the image is averaged. The optional filterpar argument determines how strong the profile is smoothed before the correction is applied.

remove_pixels_fourier(image,mask, angle)

Removes regular artifacts which were identified in Fourier space of the image as illustrated in Fig. 6.4. Pixels inside the mask are replaced by rotating the mask and averaging the new values.

combine_in_fourierspace (hfimage, lfimage, cutoff, width)

Combines two images in Fourier space by using an error-function transition as defined in Eq. (2.31), where width determines the transition strength $\sigma_{\text {cut }}$. For spatial frequencies smaller than cutoff the lfimage is used, for higher frequencies the hfimage is used. 


\section{A.3.2 Phase retrieval in the direct-contrast regime}

MBA (image, fresnelnumber, settings)

Single-distance phase-retrieval algorithm based on Eq. (2.8). The image has to be corrected by an empty image $I_{0}$ so that it varies around 1 . The most important parameter in settings is the regularization parameter reg_alpha. SMO (image, fresnelnumber, settings)

Single-distance phase-retrieval algorithm based on Eq. (2.15). The image has to be corrected by an empty image $I_{0}$ so that it varies around 1 . The most important parameter in settings is the ratio $\kappa=\delta / \beta$ denoted as beta_delta in the code.

BAC (image, fresnelnumber, settings)

Single-distance phase-retrieval algorithm based on Eq. (2.19). The image has to be corrected by an empty image $I_{0}$ so that it varies around 1 . For the reconstruction, reg_alpha and reg_gamma are required settings. Best practice is to first use the MBA function to determine reg_alpha followed by the choice of reg_gamma based on visual inspection. Mostly values are around reg_gamma $=0.15$ for direct-contrast images.

\section{A.3.3 Holographic phase-retrieval}

CTFrec (images, fresnelnumbers, settings)

Single- and multi-distance phase-retrieval based on Eq. (2.29) or (2.33). Images can be a cell or stack of images matching the given fresnelnumbers. Typically, results from rescale_defocus_series are used. The most important settings are lim1 and lim2 for the pure-phase assumption. If delta_beta is passed as an element of the settings structure, lim1 is ignored and the reconstruction is performed according to Eq. (2.33).

HoloTIE (images, fresnelnumbers, settings)

Phase retrieval as described in section 2.2.3. At least two images have to be passed with matching fresnelnumbers. If reg_alpha is passed as an element of the settings structure, the detector phase is reconstructed using regularized inverse Laplacians. If more than two images are passed, the multidistance fitting approach based on [178] is used. If a lowfrequencyimage is passed in settings, it will be used to calculate the detector phase as a combination in Fourier space, using combine_cutoff and combine_sigma. 
gs_multi (images, fresnelnumbers1, fresnelnumbers2, settings)

Iterative phase-retrieval based on an AP approach. The number of elements in fresnelnumbers has to match the number of images. A single image is also possible. Fresnelnumbers1 and fresnelnumbers2 can be used to incorporate information about astigmatism, where the first number describes the horizontal direction in an image. Many different constraints can be employed depending on the choice of settings passed.

hio_multi (images, fresnelnumbers 1 , fresnelnumbers 2 , settings)

Iterative phase-retrieval based on the mHIO algorithm. The usage is similar to gs_multi. The most important difference is the use of soft-projections and support information, for which supp has to be passed as an element of the settings structure.

newton_rec(images, fresnelnumber1, settings, [fresnelnumbers2])

Wrapper for the IRGN phase-retrieval method that is used similarly as the other methods presented here. Uses NewtonPhaseRec and it is depending functions implemented by Simon Maretzke.

IRP_rec(I_proj,fresnelx, angles, settings)

Implementation of Eq. (2.57) for 3D iterative phase-retrieval based on a single distance. Relies on 3D projection functions written by Aike Ruhlandt. I_proj has to be a stack of projections recorded under different tomographic angles, which are listed in angles with the unit degree. Fresnelx is the Fresnel number for all images. Additional constraints can be switched on and off in the settings structure.

\section{A.3.4 Functions useful for tomography}

ringremove (sino, ringparameter, $[$ range $=f u l l]$ )

Ring-removal algorithm based on the Fourier filtering approach described in section 3.2.1. A sinogram has to be passed, where the second dimension is the angular dependence. The ringparameter determines the strength of the lowpass filter. The larger it is chosen, the smaller is the effect of ring removal. By passing a range the rings can be removed for a limited range of angles.

ringremove_wavelet (sino, settings)

Ring-removal algorithm based on the wavelet approach introduced by [115]. Most important settings are decNum to determine how much wavelet com- 
ponents should be filtered, wname to choose the wavelet type and sigma to set the filter width.

pixelsize (imnumbers, reldistances, empty)

Helper function that calculates a pixel size using a lateral scan. Relies on the p10_read function to be initialized. imnumbers has to be an array of the image numbers in the scan, which are separated by reldistances. If an image is passed as empty, all images are divided by it prior to the calculation.

align_rotaxis (imnumber 1 , imnumber 2 , empty)

Function to calculate the necessary shift of two images (which again have to be read in by p10_read) so that after shifting by this value, the rotation axis is centered in the detector. If an image is passed as empty, the images are divided by it prior to the calculation.

s2m_align_nick(imnumber1, imnumber2)

Function to facilitate alignment of the pitch angle, which is used by a spec macro.

astra_coneproj (vol, angles, $\mathrm{x} 01, \mathrm{x} 02, \mathrm{p}, \mathrm{p}$ _eff , settings)

Wrapper function for the cone-beam projection provided by the astra toolbox [134]. A 3D volume vol is projected for every angle given in angles using the geometry defined by the remaining parameters. The calculation can only be performed on a GPU.

astraFDK (projs, angles , $x 01, x 02, p, p_{-}$eff , settings)

FDK reconstruction using a GPU implementation provided by the astra toolbox [134]. The use of the function is similar to astra_coneproj. The calculation can only be performed on a GPU. 



\section{Bibliography}

[1] Princeton Instruments - LCX: 1300 datasheet. http://www.princetoninstruments.com/Uploads/Princeton/Documents/ Datasheets/Princeton_Instruments_PI_LCX_1300_Rev_C1.pdf, 2015.

[2] L. Allen and M. Oxley. Phase retrieval from series of images obtained by defocus variation. Opt. Commun., 199:65-75, 2001.

[3] J. Als-Nielsen and D. McMorrow. Elements of Modern X-ray Physics. 2nd edition edition, 2011.

[4] K. Amunts, C. Lepage, L. Borgeat, H. Mohlberg, T. Dickscheid, M.-E. Rousseau, S. Bludau, P.-L. Bazin, L. B. Lewis, A.-M. Oros-Peusquens, N. J. Shah, T. Lippert, K. Zilles, and A. C. Evans. Bigbrain: An ultrahighresolution 3d human brain model. Science, 340(6139):1472-1475, 2013.

[5] A. Andersen and A. C. Kak. Simultaneous algebraic reconstruction technique (sart): a superior implementation of the art algorithm. Ultrason. Imaging, 6(1):81-94, 1984.

[6] Andor. Zyla scmos fiber-optic. http://www.andor.com/scientificcameras/high-energy-detection/zyla-scmos-hf, 2015.

[7] A. Authier. Dynamical theory of X-ray diffraction. Springer, 2006.

[8] C. T. Badea, K. K. Athreya, G. Espinosa, D. Clark, A. P. Ghafoori, Y. Li, D. G. Kirsch, G. A. Johnson, A. Annapragada, and K. B. Ghaghada. Computed tomography imaging of primary lung cancer in mice using a liposomaliodinated contrast agent. PLoS ONE, 7(4):e34496, 042012.

[9] A. Bakushinskii. The problem of the convergence of the iteratively regularized gauss-newton method. Computational Mathematics and Mathematical Physics, 32(9):1353-1359, 1992.

[10] J. Balhara and A. S. Gounni. The alveolar macrophages in asthma: a doubleedged sword. Mucosal Immunol., 5:605-609, 2012.

[11] B.-R. Bang, E. Chun, E.-J. Shim, H.-S. Lee, S.-Y. Lee, S.-H. Cho, K.-U. Min, Y.-Y. Kim, and H.-W. Park. Alveolar macrophages modulate allergic inflammation in a murine model of asthma. Exp. Mol. Med., 43:275-280, May 2011. 
[12] R. Barrett, R. Baker, P. Cloetens, Y. Dabin, C. Morawe, H. Suhonen, R. Tucoulou, A. Vivo, and L. Zhang. Dynamically-figured mirror system for highenergy nanofocusing at the ESRF. SPIE Proceedings of SPIE, 8139, 2011.

[13] M. Bartels. Phasenkontrast-Mikrotomographie an einer Laborröntgenquelle. Master's thesis, Universität Göttingen, 2010.

[14] M. Bartels. Cone-beam x-ray phase contrast tomography of biological samples Optimization of contrast, resolution and field of view. $\mathrm{PhD}$ thesis, Universität Göttingen, 2013.

[15] M. Bartels, V. H. Hernandez, M. Krenkel, T. Moser, and T. Salditt. Phase contrast tomography of the mouse cochlea at microfocus x-ray sources. Appl. Phys. Lett., 103(8):083703, 2013.

[16] M. Bartels, M. Krenkel, J. Haber, R. N. Wilke, and T. Salditt. X-ray holographic imaging of hydrated biological cells in solution. Phys. Rev. Lett., 114:048103, Jan 2015.

[17] M. Bartels, M. Priebe, R. N. Wilke, S. Krüger, K. Giewekemeyer, S. Kalbfleisch, C. Olendrowitz, C, M. Sprung, and T. Salditt. Low-dose three-dimensional hard x-ray imaging of bacterial cells. Opt. Nanoscopy, 1(1):10, 2012.

[18] A. Barty, S. Marchesini, H. N. Chapman, C. Cui, M. R. Howells, D. A. Shapiro, A. M. Minor, J. C. H. Spence, U. Weierstall, J. Ilavsky, A. Noy, S. P. Hau-Riege, A. B. Artyukhin, T. Baumann, T. Willey, J. Stolken, T. van Buuren, and J. H. Kinney. Three-Dimensional Coherent X-Ray Diffraction Imaging of a Ceramic Nanofoam: Determination of Structural Deformation Mechanisms. Phys. Rev. Lett., 101(5):055501, July 2008.

[19] H. H. Bauschke, P. L. Combettes, and D. R. Luke. Hybrid projectionreflection method for phase retrieval. J. Opt. Soc. Am. A, 20(6):1025-1034, 2003.

[20] A. Bieniek and A. Moga. An efficient watershed algorithm based on connected components. Pattern Recognition, 33(6):907 - 916, 2000.

[21] G. Binnig and H. Rohrer. Scanning tunneling microscopy. Surface Science, 126:236 - 244, 1983.

[22] K. T. Block, M. Uecker, and J. Frahm. Undersampled radial mri with multiple coils. iterative image reconstruction using a total variation constraint. Magn. Reson. Med., 57(6):1086-1098, 2007.

[23] F. E. Boas and D. Fleischmann. Ct artifacts: causes and reduction techniques. Imaging in Medicine, 4(2):229-240, Apr. 2012. 
[24] M. N. Boone, W. Devulder, M. Dierick, L. Brabant, E. Pauwels, and L. V. Hoorebeke. Comparison of two single-image phase-retrieval algorithms for in-line x-ray phase-contrast imaging. J. Opt. Soc. Am. A, 29(12):2667-2672, Dec 2012.

[25] M. Born and E. Wolf. Principles of optics: electromagnetic theory of propagation, interference and diffraction of light. Cambridge University Press, 1999.

[26] C. Broennimann, E. F. Eikenberry, B. Henrich, R. Horisberger, G. Huelsen, E. Pohl, B. Schmitt, C. Schulze-Briese, M. Suzuki, T. Tomizaki, H. Toyokawa, and A. Wagner. The PILATUS 1M detector. J. Synchrotron Rad., 13(2):120-130, Mar 2006.

[27] A. V. Bronnikov. Cone-beam reconstruction by backprojection and filtering. J. Opt. Soc. Am. A, 17(11):1993-2000, Nov 2000.

[28] A. Burvall, U. Lundström, P. A. C. Takman, D. H. Larsson, and H. M. Hertz. Phase retrieval in X-ray phase-contrast imaging suitable for tomography. Opt. Express, 19(11):10359-10376, May 2011.

[29] T. Buzug. Computed Tomography : From Photon Statistics to Modern ConeBeam CT. Springer-Verlag, 2008.

[30] C. L. Byrne. Applied iterative methods. AK Peters Wellesley, 2008.

[31] W. Chao, B. D. Harteneck, J. A. Liddle, E. H. Anderson, and D. T. Attwood. Soft X-ray microscopy at a spatial resolution better than $15 \mathrm{~nm}$. Nature, 435(7046):1210-1213, June 2005.

[32] H. N. Chapman, C. Caleman, and N. Timneanu. Diffraction before destruction. Philos T Roy Soc B, 369(1647):20130313, 2014.

[33] H.-Y. Chen, S. Hoffmann, and T. Salditt. X-ray beam compression by tapered waveguides. Applied Physics Letters, 106(19):-, 2015.

[34] C. Chien, P. Tseng, H. Chen, T. Hua, S. Chen, Y. Chen, W. Leng, C. Wang, Y. Hwu, G. Yin, K. Liang, F. Chen, Y. Chu, H. Yeh, Y. Yang, C. Yang, G. Zhang, J. Je, and G. Margaritondo. Imaging cells and sub-cellular structures with ultrahigh resolution full-field x-ray microscopy. Biotechnol. Adv., 31(3):375 - 386, 2013. High-Resolution multimodal imaging of living systems.

[35] P. Cloetens. Contribution to Phase Contrast Imaging, Reconstruction and Tomography with Hard Synchrotron Radiation. PhD thesis, Vrije Universiteit Brussel, 1999. 
[36] P. Cloetens, R. Barrett, J. Baruchel, J.-P. Guigay, and M. Schlenker. Phase objects in synchrotron radiation hard x-ray imaging. Journal of Physics D: Applied Physics, 29(1):133-146, 1996.

[37] P. Cloetens, W. Ludwig, J. Baruchel, D. Van Dyck, J. Van Landuyt, J. P. Guigay, and M. a. Schlenker. Holotomography: Quantitative phase tomography with micrometer resolution using hard synchrotron radiation $\mathrm{x}$ rays. Appl. Phys. Lett., 75(19):2912-2914, 1999.

[38] A. J. D’Alfonso, A. J. Morgan, A. W. C. Yan, P. Wang, H. Sawada, A. I. Kirkland, and L. J. Allen. Deterministic electron ptychography at atomic resolution. Phys. Rev. B, 89(6):064101, Feb. 2014.

[39] C. David, B. Nohammer, H. H. Solak, and E. Ziegler. Differential x-ray phase contrast imaging using a shearing interferometer. Appl. Phys. Lett., 81(17):3287-3289, Oct. 2002.

[40] P. J. Delves, S. J. Martin, D. R. Burton, and I. M. Roitt. Roitt's Essential Immunology, volume 20. John Wiley \& Sons, 2006.

[41] M. Dierolf, A. Menzel, P. Thibault, P. Schneider, C. M. Kewish, R. Wepf, O. Bunk, and F. Pfeiffer. Ptychographic X-ray computed tomography at the nanoscale. Nature, 467(7314):436-439, Sept. 2010.

[42] P.-A. Douissard, A. Cecilia, T. Martin, V. Chevalier, M. Couchaud, T. Baumbach, K. Dupré, M. Kühbacher, and A. Rack. A novel epitaxially grown LSO-based thin-film scintillator for micro-imaging using hard synchrotron radiation. J. Synchrotron Rad., 17(5):571-583, Sep 2010.

[43] C. Dullin, S. dal Monego, E. Larsson, S. Mohammadi, M. Krenkel, C. Garrovo, S. Biffi, A. Lorenzon, A. Markus, J. Napp, T. Salditt, A. Accardo, F. Alves, and G. Tromba. Functionalized synchrotron in-line phase-contrast computed tomography: a novel approach for simultaneous quantification of structural alterations and localization of barium-labelled alveolar macrophages within mouse lung samples. J. Synchrotron Rad., 22(1):143-155, Jan 2015 .

[44] ESRF. Conceptual design report: Upbl4 nano-imaging and nano-analysis. Technical report, ESRF Upgrade Programme 300409, 2015.

[45] L. A. Feldkamp, L. C. Davis, and J. W. Kress. Practical cone-beam algorithm. J. Opt. Soc. Am. A, 1(6):612-619, Jun 1984.

[46] J. R. Fienup. Phase retrieval algorithms: a comparison. Appl. Opt., 21(15):2758-2769, 1982. 
[47] R. Fletcher. Conjugate gradient methods for indefinite systems. In Numerical analysis, pages 73-89. Springer, 1976.

[48] B. Fowler, C. Liu, S. Mims, J. Balicki, W. Li, H. Do, J. Appelbaum, and P. Vu. A 5.5mpixel 100 frames/sec wide dynamic range low noise cmos image sensor for scientific applications. volume 7536 , pages $753607-753607-$ $12,2010$.

[49] H. Franz, O. Leupold, R. Röhlsberger, S. Roth, O. Seeck, J. Spengler, J. Strempfer, M. Tischer, J. Viefhaus, E. Weckert, and T. Wroblewski. Technical report: Petra iii: Desy's new high brilliance third generation synchrotron radiation source. Synch. Rad. News, 19(6):25-29, 2006.

[50] W. Friedrich, P. Knipping, and M. Laue. Interferenzerscheinungen bei Röntgenstrahlen. Annalen der Physik, 346(10):971-988, 1913.

[51] D. Gabor. A New Microscopic Principle. Nature, 161:777-778, May 1948.

[52] R. W. Gerchberg and W. O. Saxton. A Practical Algorithm for the Determination of Phase from Image and Diffraction Plane Pictures. Optik, 35(2):237-246, November 1972.

[53] K. Giewekemeyer. A study on new approaches in coherent x-ray microscopy of biological specimens. PhD thesis, Universität Göttingen, 2011.

[54] K. Giewekemeyer, S. P. Krüger, S. Kalbfleisch, M. Bartels, C. Beta, and T. Salditt. X-ray propagation microscopy of biological cells using waveguides as a quasipoint source. Phys. Rev. A, 83(2):023804, Feb 2011.

[55] J. W. Goodman. Introduction to Fourier Optics. Roberts \& Company: Englewood, Colorado, 2005.

[56] R. Gordon, R. Bender, and G. T. Herman. Algebraic reconstruction techniques (art) for three-dimensional electron microscopy and x-ray photography. Journal of Theoretical Biology, 29(3):471 - 481, 1970.

[57] A. Groso, R. Abela, and M. Stampanoni. Implementation of a fast method for high resolution phase contrast tomography. Opt. Express, 14(18):81038110, Sept. 2006.

[58] G. Gstraunthaler and T. Lindl. Zell-und Gewebekultur. Springer, 2013.

[59] J. P. Guigay. Fourier transform analysis of fresnel diffraction patterns and in-line holograms. Optik, 49(1):121-125, 1977.

[60] M. Guizar-Sicairos, J. J. Boon, K. Mader, A. Diaz, A. Menzel, and O. Bunk. Quantitative interior x-ray nanotomography by a hybrid imaging technique. Optica, 2(3):259-266, Mar 2015. 
[61] M. Guizar-Sicairos, A. Diaz, M. Holler, M. S. Lucas, A. Menzel, R. A. Wepf, and O. Bunk. Phase tomography from x-ray coherent diffractive imaging projections. Opt. Express, 19(22):21345-21357, Oct. 2011.

[62] M. Guizar-Sicairos, S. T. Thurman, and J. R. Fienup. Efficient subpixel image registration algorithms. Opt. Lett., 33(2):156-158, Jan. 2008.

[63] T. E. Gureyev. Composite techniques for phase retrieval in the Fresnel region. Opt. Commun., 220(1-3):49 - 58, 2003.

[64] T. E. Gureyev, A. Roberts, and K. A. Nugent. Phase retrieval with the transport-of-intensity equation: matrix solution with use of Zernike polynomials. J. Opt. Soc. Am. A, 12(9):1932, 1995.

[65] J. Hagemann, A.-L. Robisch, D. R. Luke, C. Homann, T. Hohage, P. Cloetens, H. Suhonen, and T. Salditt. Reconstruction of wave front and object for inline holography from a set of detection planes. Opt. Express, 22(10):11552-11569, May 2014.

[66] Hamamatsu. Fiber optic plates - direct image transmission. http://www.hamamatsu.com/resources/pdf/etd/FOP_TMCP1040E.pdf, 2015 .

[67] Hamamatsu. Orca-flash4.0 v2 - digital cmos camera. http://www.hamamatsu.com/resources/pdf/sys/SCAS0081E_C1144022CU.pdf, 2015.

[68] M. v. Heel. Similarity measures between images. Ultramicroscopy, 21(1):95$100,1987$.

[69] S. Helgason. The radon transform on euclidean spaces, compact twopoint homogeneous spaces and grassmann manifolds. Acta Mathematica, 113(1):153-180, 1965.

[70] S. W. Hell. Far-Field Optical Nanoscopy. Science, 316(5828):1153-1158, 2007.

[71] B. L. Henke, E. M. Gullikson, and J. C. Davis. X-Ray Interactions: Photoabsorption, Scattering, Transmission, and Reflection at $\mathrm{E}=50-30,000 \mathrm{eV}$, $\mathrm{Z}=1$-92. At. Data Nucl. Data Tables, 54(2):181-342, July 1993.

[72] B. Henrich, J. Becker, R. Dinapoli, P. Goettlicher, H. Graafsma, H. Hirsemann, R. Klanner, H. Krueger, R. Mazzocco, A. Mozzanica, H. Perrey, G. Potdevin, B. Schmitt, X. Shi, A. Srivastava, U. Trunk, and C. Youngman. The adaptive gain integrating pixel detector AGIPD a detector for the European XFEL. Nucl Instrum Methods Phys Res, Sect A, 633(Supplement 1):S11-S14, May 2011. 
[73] R. Hesse. Fixed Point Algorithms for Nonconvex Feasibility with Applications. PhD thesis, Universität Göttingen, 2014.

[74] T. Hohage. Logarithmic convergence rates of the iteratively regularized gauss - newton method for an inverse potential and an inverse scattering problem. Inverse Prob., 13(5):1279, 1997.

[75] C. Holness and D. Simmons. Molecular cloning of cd68, a human macrophage marker related to. Blood, 81(6):1607-1613, 1993.

[76] C. Homann, T. Hohage, J. Hagemann, A.-L. Robisch, and T. Salditt. Validity of the empty-beam correction in near-field imaging. Phys. Rev. A, 91:013821, Jan 2015.

[77] G. N. Hounsfield. Computerized transverse axial scanning (tomography): Part 1. description of system. The British Journal of Radiology, 46(552):1016-1022, 1973. PMID: 4757352.

[78] M. Howells, T. Beetz, H. Chapman, C. Cui, J. Holton, C. Jacobsen, J. Kirz, E. Lima, S. Marchesini, H. Miao, D. Sayre, D. Shapiro, J. Spence, and D. Starodub. An assessment of the resolution limitation due to radiationdamage in X-ray diffraction microscopy. Journal of Electron Spectroscopy and Related Phenomena, 170(1-3):4-12, Mar. 2009.

[79] J. D. Jackson. Classical electrodynamics. Wiley, 1999.

[80] D. A. Jaffray, J. H. Siewerdsen, J. W. Wong, and A. A. Martinez. Flatpanel cone-beam computed tomography for image-guided radiation therapy. International Journal of Radiation Oncology*Biology*Physics, 53(5):1337 $1349,2002$.

[81] T. H. Jensen, M. Bech, O. Bunk, T. Donath, C. David, R. Feidenhans, and F. Pfeiffer. Directional x-ray dark-field imaging. Physics in medicine and biology, 55(12):3317, 2010.

[82] H. Jiang, C. Song, C.-C. Chen, R. Xu, K. S. Raines, B. P. Fahimian, C.H. Lu, T.-K. Lee, A. Nakashima, J. Urano, T. Ishikawa, F. Tamanoi, and J. Miao. Quantitative 3D imaging of whole, unstained cells by using X-ray diffraction microscopy. PNAS, 107(25):11234-11239, 2010.

[83] S. Kaczmarz. Angenäherte Aufösung von Systemen linearer Gleichungen. Bull. Acad. Polon. Sci. Lett., A35:355-357, 1937.

[84] A. C. Kak and M. Slaney. Principles of computerized tomographic imaging. IEEE Press, New York, 1988.

[85] S. Kalbfleisch. A Dedicated Endstation for Waveguide-based X-Ray Imaging. PhD thesis, Universität Göttingen, 2012. 
[86] D. G. Kirsch, J. Grimm, A. R. Guimaraes, G. R. Wojtkiewicz, B. A. Perez, P. M. Santiago, N. K. Anthony, T. Forbes, K. Doppke, R. Weissleder, and T. Jacks. Imaging primary lung cancers in mice to study radiation biology. International Journal of Radiation Oncology Biology Physics, 76(4):973 977, 2010.

[87] M. Krenkel. Quantitative Phasenkontrast Tomographie. Master's thesis, Universität Göttingen, 2012.

[88] M. Krenkel, M. Bartels, and T. Salditt. Transport of intensity phase reconstruction to solve the twin image problem in holographic x-ray imaging. Opt. Express, 21(2):2220-2235, Jan. 2013.

[89] M. Krenkel, A. Markus, M. Bartels, C. Dullin, F. Alves, and T. Salditt. Phase-contrast zoom tomography reveals precise locations of macrophages in mouse lungs. Sci. Rep., 5:-, May 2015.

[90] S. P. Krüger. Optimization of waveguide optics for lensless x-ray imaging. $\mathrm{PhD}$ thesis, Universität Göttingen, 2010.

[91] S. P. Krüger, K. Giewekemeyer, S. Kalbfleisch, M. Bartels, H. Neubauer, and T. Salditt. Sub-15 nm beam confinement by twocrossed x-ray waveguides. Opt. Express, 18(13):13492-13501, June 2010.

[92] S. P. Krüger, H. Neubauer, M. Bartels, S. Kalbfleisch, K. Giewekemeyer, P. J. Wilbrandt, M. Sprung, and T. Salditt. Sub-10 nm beam confinement by X-ray waveguides: design, fabrication and characterization of optical properties. J. Synchrotron Rad., 19(2):227-236, 2012.

[93] H. Kudo and T. Saito. Sinogram recovery with the method of convex projections for limited-data reconstruction in computed tomography. J. Opt. Soc. Am. A, 8(7):1148-1160, Jul 1991.

[94] A. Kyrieleis, V. Titarenko, M. Ibison, T. Connolley, and P. Withers. Regionof-interest tomography using filtered backprojection: assessing the practical limits. Journal of microscopy, 241(1):69-82, 2011.

[95] J.-C. Labiche, O. Mathon, S. Pascarelli, M. A. Newton, G. G. Ferre, C. Curfs, G. Vaughan, A. Homs, and D. F. Carreiras. Invited article: The fast readout low noise camera as a versatile x-ray detector for time resolved dispersive extended x-ray absorption fine structure and diffraction studies of dynamic problems in materials science, chemistry, and catalysis. Rev. Sci. Instrum., 78(9):091301-11, Sept. 2007.

[96] B. Laforce, S. Schmitz, B. Vekemans, J. Rudloff, J. Garrevoet, R. Tucoulou, F. E. Brenker, G. Martinez-Criado, and L. Vincze. Nanoscopic x-ray fluores- 
cence imaging of meteoritic particles and diamond inclusions. Anal. Chem., 86(24):12369-12374, 2014. PMID: 25395119.

[97] C. A. Larabell and K. A. Nugent. Imaging cellular architecture with X-rays. Curr. Opin. Struct. Biol., 20(5):623 - 631, 2010.

[98] D. Larsson. Small-Animal Imaging with Liquid-Metal-Jet X-ray Sources. PhD thesis, KTH Royal institute of technology, 2015.

[99] H. Liu, Z. Xu, X. Zhang, Y. Wu, Z. Guo, and R. Tai. Effects of missing lowfrequency information on ptychographic and plane-wave coherent diffraction imaging. Appl. Opt., 52(11):2416-2427, Apr. 2013.

[100] X. Llopart, M. Campbell, R. Dinapoli, D. San Segundo, and E. Pernigotti. Medipix2: A $64 \mathrm{k}$ pixel readout chip with $55 \mu \mathrm{m}$ square elements working in single photon counting mode. Nuclear Science, IEEE Transactions, 49(5):2279-2283, 2002.

[101] D. Ludwig. The radon transform on euclidean space. Commun. Pure Appl. Math., 19(1):49-81, 1966.

[102] D. R. Luke. Relaxed averaged alternating reflections for diffraction imaging. Inverse Problems, 21(1):37, 2005.

[103] S. Maretzke. Regularized newton methods for simultaneous radon inversion and phase retrieval in phase contrast tomography. Master's thesis, GeorgAugust-University Göttingen, 2015.

[104] M. A. Markus, C. Dullin, M. Mitkovski, E. Prieschl-Grassauer, M. M. Epstein, and F. Alves. Non-invasive optical imaging of eosinophilia during the course of an experimental allergic airways disease model and in response to therapy. PLoS ONE, 9(2):e90017, 022014.

[105] F. D. Martinez. Genes, environments, development and asthma: a reappraisal. Eur. Respir. J., 29(1):179-184, 2007.

[106] G. Martinez-Criado, R. Tucoulou, P. Cloetens, P. Bleuet, S. Bohic, J. Cauzid, I. Kieffer, E. Kosior, S. Laboure, S. Petitgirard, A. Rack, J. A. Sans, J. Segura-Ruiz, H. Suhonen, J. Susini, and J. Villanova. Status of the hard $\mathrm{x}$-ray microprobe beamline id22 of the european synchrotron radiation facility. J. Synchrotron Rad., 19(1):10-18, 2012.

[107] I. N. Mbawuike and H. B. Herscowitz. MH-S, a murine alveolar macrophage cell line: morphological, cytochemical, and functional characteristics. $J$. Leukoc. Biol., 46(2):119-127, 1989.

[108] B. F. McEwen, K. H. Downing, and R. M. Glaeser. The relevance of 
dose-fractionation in tomography of radiation-sensitive specimens. Ultramicroscopy, 60(3):357-373, Oct. 1995.

[109] J. Miao, P. Charalambous, J. Kirz, and D. Sayre. Extending the methodology of X-ray crystallography to allow imaging of micrometre-sized noncrystalline specimens. Nature, 400(6742):342-344, July 1999.

[110] Y. Mizue, S. Ghani, L. Leng, C. McDonald, P. Kong, J. Baugh, S. J. Lane, J. Craft, J. Nishihira, S. C. Donnelly, Z. Zhu, and R. Bucala. Role for macrophage migration inhibitory factor in asthma. Proceedings of the $\mathrm{Na}$ tional Academy of Sciences, 102(40):14410-14415, 2005.

[111] A. Momose, T. Takeda, Y. Itai, and K. Hirano. Phase-contrast x-ray computed tomography for observing biological soft tissues. Nat. Med., 2(4):473475, Apr. 1996.

[112] W. D. Montgomery. Unitary operators in the homogeneous wave field. Opt. Lett., 6(7):314-315, Jul 1981.

[113] J. Moosmann, A. Ershov, V. Altapova, T. Baumbach, M. S. Prasad, C. LaBonne, X. Xiao, J. Kashef, and R. Hofmann. X-ray phase-contrast in vivo microtomography probes new aspects of xenopus gastrulation. $\mathrm{Na}$ ture, 497(497):374 - 377, 052013.

[114] A. P. Moreira and C. M. Hogaboam. Macrophages in allergic asthma: Finetuning their pro- and anti-inflammatory actions for disease resolution. $J$. Interferon Cytokine Res., 31:485-491, June 2011.

[115] B. Münch, P. Trtik, F. Marone, and M. Stampanoni. Stripe and ring artifact removal with combined wavelet-fourier filtering. Opt. Express, 17(10):85678591, 2009.

[116] F. Natterer. The mathematics of computerized tomography. Classics in Applied Mathematics. Society for Industrial and Applied Mathematics, Philadelphia, PA, USA, 2001.

[117] C. Nave. Radiation damage in protein crystallography. Radiat. Phys. Chem., 45(3):483-490, 1995.

[118] P. D. Nellist and J. M. Rodenburg. Electron ptychography. i. experimental demonstration beyond the conventional resolution limits. Acta Crystallographica Section A, 54(1):49-60, 1998.

[119] J. Nelson, X. Huang, J. Steinbrener, D. Shapiro, J. Kirz, S. Marchesini, A. M. Neiman, J. J. Turner, and C. Jacobsen. High-resolution x-ray diffraction microscopy of specifically labeled yeast cells. PNAS, 107(16):7235-7239, Apr. 2010. 
[120] H. Neubauer, S. Hoffmann, M. Kanbach, J. Haber, S. Kalbfleisch, S. P. Krüger, and T. Salditt. High aspect ratio x-ray waveguide channels fabricated by e-beam lithography and wafer bonding. J. Appl. Phys., 115(21):214305, 2014.

[121] V. V. Nieuwenhove, J. D. Beenhouwer, T. D. Schryver, L. V. Hoorebeke, and J. Sijbers. Affine deformation correction in cone beam computed tomography. In Fully Three-Dimensional Image Reconstruction in Radiology and Nuclear Medicine, pages 182-185, Newport, 06/2015 2015.

[122] Y. Nishino, Y. Takahashi, N. Imamoto, T. Ishikawa, and K. Maeshima. Three-Dimensional Visualization of a Human Chromosome Using Coherent X-Ray Diffraction. Phys. Rev. Lett., 102:018101, Jan 2009.

[123] K. A. Nugent. Partially coherent diffraction patterns and coherence measurement. J. Opt. Soc. Am. A, 8(10):1574-1579, Oct. 1991.

[124] K. A. Nugent. Coherent methods in the X-ray sciences. Adv. Phys., 59(1):199, 2010.

[125] K. A. Nugent, T. E. Gureyev, D. F. Cookson, D. Paganin, and Z. Barnea. Quantitative Phase Imaging Using Hard X Rays. Phys. Rev. Lett., 77(14):2961-2964, Sep 1996.

[126] C. Olendrowitz, M. Bartels, M. Krenkel, A. Beerlink, R. Mokso, M. Sprung, and T. Salditt. Phase-contrast x-ray imaging and tomography of the nematode Caenorhabditis elegans. Phys. Med. Biol., 57(16):5309, 2012.

[127] M. Osterhoff. Wave optical simulations of x-ray nano-focusing optics. $\mathrm{PhD}$ thesis, 2012.

[128] M. Otendal, T. Tuohimaa, and H. M. Hertz. Stability and debris in highbrightness liquid-metal-jet-anode microfocus x-ray sources. J. Appl. Phys., 101(2):026102, 2007.

[129] M. Otendal, T. Tuohimaa, U. Vogt, and H. M. Hertz. A 9 keV electronimpact liquid-gallium-jet x-ray source. Rev. Sci. Instrum., 79(1):016102, 2008.

[130] C. H. Owen and W. J. Landis. Alignment of electron tomographic series by correlation without the use of gold particles. Ultramicroscopy, 63(1):27 - 38, 1996.

[131] D. Paganin, S. C. Mayo, T. E. Gureyev, P. R. Miller, and S. W. Wilkins. Simultaneous phase and amplitude extraction from a single defocused image of a homogeneous object. J. Microsc., 206(Pt 1):33-40, 2002. 
[132] D. Paganin and K. A. Nugent. Noninterferometric Phase Imaging with Partially Coherent Light. Phys. Rev. Lett., 80:2586-2589, Mar 1998.

[133] D. M. Paganin. Coherent X-Ray Optics. New York: Oxford University Press, 2006.

[134] W. J. Palenstijn, K. J. Batenburg, and J. Sijbers. The astra tomography toolbox. In 13th International Conference on Computational and Mathematical Methods in Science and Engineering. CMMSE, 2013.

[135] D. L. Parker. Optimal short scan convolution reconstruction for fan beam ct. Medical Physics, 9(2):254-257, 1982.

[136] A. Peterzol, A. Olivo, L. Rigon, S. Pani, and D. Dreossi. The effects of the imaging system on the validity limits of the ray-optical approach to phase contrast imaging. Med. Phys., 32(12):3617-3627, 2005.

[137] F. Pfeiffer, M. Bech, O. Bunk, P. Kraft, E. F. Eikenberry, C. Brönnimann, C. Grünzweig, and C. David. Hard-x-ray dark-field imaging using a grating interferometer. Nature Materials, 7:134-137, 2008.

[138] F. Pfeiffer, C. Kottler, O. Bunk, and C. David. Hard X-Ray Phase Tomography with Low-Brilliance Sources. Phys. Rev. Lett., 98:108105, Mar 2007.

[139] B. Pinzer, M. Cacquevel, P. Modregger, S. McDonald, J. Bensadoun, T. Thuering, P. Aebischer, and M. Stampanoni. Imaging brain amyloid deposition using grating-based differential phase contrast tomography. NeuroImage, 61(4):1336 - 1346, 2012.

[140] A. Pogany, D. Gao, and S. W. Wilkins. Contrast and resolution in imaging with a microfocus x-ray source. Rev. Sci. Instrum., 68(7):2774-2782, 1997.

[141] C. Ponchut, J. Clément, J.-M. Rigal, E. Papillon, J. Vallerga, D. LaMarra, and B. Mikulec. Photon-counting X-ray imaging at kilohertz frame rates. Nucl. Instrum. Methods Phys. Res., 576(1):109-112, June 2007.

[142] W. H. Press, B. P. Flannery, S. A. Teukolsky, and W. T. Vetterling. Numerical recipes. Cambridge university press Cambridge, 1990.

[143] J. Radon. Über die Bestimmung von Funktionen durch ihre Integralwerte längs gewisser Mannigfaltigkeiten. Akad. Wiss., 69:262-277, 1917.

[144] G. Ramachandran and A. Lakshminarayanan. Three-dimensional reconstruction from radiographs and electron micrographs: application of convolutions instead of fourier transforms. PNAS, 68(9):2236-2240, 1971.

[145] Rastrojo. Diagrama de los pulmones. Creative Commons BY-SA 4.0-3.02.5-2.0-1.0, 30.06.2015 2015. 
[146] A.-L. Robisch and T. Salditt. Phase retrieval for object and probe using a series of defocus near-field images. Opt. Express, 21(20):23345-23357, Oct 2013.

[147] J. M. Rodenburg and H. M. L. Faulkner. A phase retrieval algorithm for shifting illumination. Appl. Phys. Lett., 85(20):4795-4797, Nov. 2004.

[148] J. M. Rodenburg, A. C. Hurst, A. G. Cullis, B. R. Dobson, F. Pfeiffer, O. Bunk, C. David, K. Jefimovs, and I. Johnson. Hard-X-Ray Lensless Imaging of Extended Objects. Phys. Rev. Lett., 98(3):034801, Jan. 2007.

[149] M. Roming, H. Lünsdorf, K. E. J. Dittmar, and C. Feldmann. Zro(hpo4)(1$\mathrm{x})(\mathrm{fmn}) \mathrm{x}$ : schnelle und einfache synthese eines nanoskaligen lumineszenzbiomarkers. Angewandte Chemie, 122(3):642-647, 2010.

[150] W. Röntgen. Über eine neue Art von Strahlen. Sitzungsberichte der physikal.medizin. Gesellschaft, page 132, 1895.

[151] A. Ruhlandt, M. Krenkel, M. Bartels, and T. Salditt. Three-dimensional phase retrieval in propagation-based phase-contrast imaging. Phys. Rev. A, 89:033847, Mar 2014.

[152] C. Ryan, D. Siddons, R. Kirkham, P. Dunn, A. Kuczewski, G. Moorhead, G. De Geronimo, D. Paterson, M. De Jonge, R. Hough, et al. The new maia detector system: methods for high definition trace element imaging of natural material. In AIP Conference Proceedings, volume 1221, page 9, 2010.

[153] T. Salditt, K. Giewekemeyer, C. Fuhse, S. P. Kruger, R. Tucoulou, and P. Cloetens. Projection phase contrast microscopy with a hard x-ray nanofocused beam: Defocus and contrast transfer. Phys. Rev. B, 79(18):184112-6, May 2009.

[154] T. Salditt, M. Osterhoff, M. Krenkel, R. N. Wilke, M. Priebe, M. Bartels, S. Kalbfleisch, and M. Sprung. Compound focusing mirror and X-ray waveguide optics for coherent imaging and nano-diffraction. J. Synchrotron Rad., 22(4):867-878, Jul 2015.

[155] S. J. Schambach, S. Bag, L. Schilling, C. Groden, and M. A. Brockmann. Application of micro-ct in small animal imaging. Methods, 50(1):2 - 13, 2010. Small animal micro-CT imaging.

[156] G. Schmahl, D. Rudolph, B. Niemann, and O. Christ. Zone-plate X-ray microscopy. Q. Rev. Biophys., 13(3):297-315, 1980.

[157] A. Schropp, P. Boye, J. M. Feldkamp, R. Hoppe, J. Patommel, D. Samberg, S. Stephan, K. Giewekemeyer, R. N. Wilke, T. Salditt, J. Gulden, A. P. 
Mancuso, I. A. Vartanyants, E. Weckert, S. Schoder, M. Burghammer, and C. G. Schroer. Hard x-ray nanobeam characterization by coherent diffraction microscopy. Appl. Phys. Lett., 96(9):091102, Mar. 2010.

[158] A. Schropp, R. Hoppe, J. Patommel, D. Samberg, F. Seiboth, S. Stephan, G. Wellenreuther, G. Falkenberg, and C. G. Schroer. Hard x-ray scanning microscopy with coherent radiation: Beyond the resolution of conventional x-ray microscopes. Appl. Phys. Lett., 100(25):253112, June 2012.

[159] L. A. Shepp and B. F. Logan. The Fourier reconstruction of a head section. IEEE Transactions on Nuclear Science, 21(3):21-43, 1974.

[160] J. R. Shewell and E. Wolf. Inverse diffraction and a new reciprocity theorem. J. Opt. Soc. Am., 58(12):1596-1603, Dec 1968.

[161] J. H. Siewerdsen, L. E. Antonuk, Y. El-Mohri, J. Yorkston, W. Huang, and I. A. Cunningham. Signal, noise power spectrum, and detective quantum efficiency of indirect-detection flat-panel imagers for diagnostic radiology. Medical Physics, 25(5):614-628, 1998.

[162] SmarAct. Smarpod 110.45 - closed loop properties. http://smaract.de/index.php/products/smarpod/smarpod-110-45, 2015.

[163] M. Soto and E. Acosta. Improved phase imaging from intensity measurements in multiple planes. Appl. Opt., 46(33):7978-7981, Nov 2007.

[164] M. Stampanoni, A. Groso, A. Isenegger, G. Mikuljan, Q. Chen, D. Meister, M. Lange, R. Betemps, S. Henein, and R. Abela. TOMCAT: A beamline for TOmographic Microscopy and Coherent rAdiology experimenTs. AIPConf.Proc., 2007.

[165] M. Stockmar, P. Cloetens, I. Zanette, B. Enders, M. Dierolf, F. Pfeiffer, and P. Thibault. Near-field ptychography: phase retrieval for inline holography using a structured illumination. Sci. Rep., 3:1927, 2013.

[166] M. R. Teague. Deterministic phase retrieval: a Green's function solution. J. Opt. Soc. Am., 73(11):1434-1441, Nov 1983.

[167] P. Thibault. Algorithmic methods in diffraction microscopy. PhD thesis, University Microfilms International, Ann Arbor, Mich., 2008.

[168] P. Thibault, M. Dierolf, O. Bunk, A. Menzel, and F. Pfeiffer. Probe retrieval in ptychographic coherent diffractive imaging. Ultramicroscopy, 109(4):338343, Mar. 2009.

[169] P. Thibault and V. Elser. X-Ray Diffraction Microscopy. Annu. Rev. Condens. Matter Phys., 1(1):237-255, July 2010. 
[170] P. Thibault, V. Elser, J. C., S. D., and D. Sayre. Reconstruction of a yeast cell from X-ray diffraction data. Act. Cryst. A, 62:248-261, 2006.

[171] P. Thibault and A. Menzel. Reconstructing state mixtures from diffraction measurements. Nature, 494(7435):68-71, Feb. 2013.

[172] L. Turner, B. Dhal, J. Hayes, A. Mancuso, K. Nugent, D. Paterson, R. Scholten, C. Tran, and A. Peele. X-ray phase imaging: Demonstration of extended conditions for homogeneous objects. Opt. Express, 12(13):29602965, Jun 2004.

[173] M. Uecker, S. Zhang, D. Voit, A. Karaus, K.-D. Merboldt, and J. Frahm. Real-time mri at a resolution of 20 ms. NMR Biomed., 23(8):986-994, 2010.

[174] K. Uesugi, M. Hoshino, A. Takeuchi, Y. Suzuki, and N. Yagi. Development of fast and high throughput tomography using cmos image detector at spring- 8 . volume 8506, pages 85060I-85060I-9, 2012.

[175] M. van Heel, B. Gowen, R. Matadeen, E. V. Orlova, R. Finn, T. Pape, D. Cohen, H. Stark, R. Schmidt, M. Schatz, et al. Single-particle electron cryo-microscopy: towards atomic resolution. Q. Rev. Biophys., 33(04):307$369,2000$.

[176] M. van Heel and M. Schatz. Fourier shell correlation threshold criteria. J. Struct. Biol., 151(3):250 - 262, 2005.

[177] D. G. Voelz and M. C. Roggemann. Digital simulation of scalar optical diffraction: revisiting chirp function sampling criteria and consequences. Appl. Opt., 48(32):6132-6142, 2009.

[178] L. Waller, L. Tian, and G. Barbastathis. Transport of Intensity phaseamplitude imaging with higher order intensity derivatives. Opt. Express, 18(12):12552-12561, Jun 2010.

[179] T. Weitkamp, A. Diaz, C. David, F. Pfeiffer, M. Stampanoni, P. Cloetens, and E. Ziegler. X-ray phase imaging with a grating interferometer. Opt. Express, 13(16):6296-6304, Aug 2005.

[180] T. Weitkamp, D. Haas, D. Wegrzynek, and A. Rack. ANKAphase: software for single-distance phase retrieval from inline X-ray phase-contrast radiographs. J. Synchrotron Rad., 18(4):617-629, Jul 2011.

[181] W. J. Weninger and T. Mohun. Phenotyping transgenic embryos: a rapid 3-d screening method based on episcopic fluorescence image capturing. Nat. Genet., 30(1):59-65, 2002.

[182] R. N. Wilke. Zur Phasenrekonstruktion von X-Feldern mit gekrümmten Phasenfronten. Diplomarbeit, Universität Göttingen. 2010. 
[183] R. N. Wilke, M. Hoppert, M. Krenkel, M. Bartels, and T. Salditt. Quantitative X-ray phase contrast waveguide imaging of bacterial endospores. $J$. Appl. Crystallogr., 48(2):464-476, Apr 2015.

[184] R. N. Wilke, M. Priebe, M. Bartels, K. Giewekemeyer, A. Diaz, P. Karvinen, and T. Salditt. Hard X-ray imaging of bacterial cells: nano-diffraction and ptychographic reconstruction. Opt. Express, 20(17):19232-19254, Aug. 2012.

[185] S. W. Wilkins, T. E. Gureyev, D. Gao, A. Pogany, and A. W. Stevenson. Phase-contrast imaging using polychromatic hard X-rays. Nature, 384(6607):335-338, Nov. 1996.

[186] G. J. Williams, H. M. Quiney, B. B. Dhal, C. Q. Tran, K. A. Nugent, A. G. Peele, D. Paterson, and M. D. de Jonge. Fresnel Coherent Diffractive Imaging. Phys. Rev. Lett., 97(2):025506, 2006.

[187] Y. D. Witte, M. Boone, J. Vlassenbroeck, M. Dierick, and L. V. Hoorebeke. Bronnikov-aided correction for x-ray computed tomography. J. Opt. Soc. Am. A, 26(4):890-894, Apr 2009.

[188] World Health Organization. Fact sheet no 307: Asthma. 307, May 2011.

[189] J. Yang, H. Yu, M. Jiang, and G. Wang. High-order total variation minimization for interior tomography. Inverse Problems, 26(3):035013, 2010.

[190] M. Yang, R. K. Kumar, P. M. Hansbro, and P. S. Foster. Emerging roles of pulmonary macrophages in driving the development of severe asthma. $J$. Leukocyte Biol., 91(4):557-569, 2012.

[191] H. Yu and G. Wang. Compressed sensing based interior tomography. Physics in Medicine and Biology, 54(9):2791, 2009.

[192] S. Zabler, P. Cloetens, J.-P. Guigay, J. Baruchel, and M. Schlenker. Optimization of phase contrast imaging using hard x rays. Rev. Sci. Instrum., $76(7): 073705,2005$.

[193] I. Zanette, S. Lang, A. Rack, M. Dominietto, M. Langer, F. Pfeiffer, T. Weitkamp, and B. Müller. Holotomography versus x-ray grating interferometry: A comparative study. Appl. Phys. Lett., 103(24), 2013.

[194] F. Zernike. The concept of degree of coherence and its application to optical problems. Physica, 5(8):785-795, Aug. 1938. 


\section{Own publications}

- M. Krenkel, A. Markus, M. Bartels, C. Dullin, F. Alves and T. Salditt, "Phase contrast zoom-tomography reveals precise locations of macrophages in mouse lungs", Sci. Rep. 5, 09973 (2015).

- M. Krenkel, M. Töpperwien, M. Bartels, P. Lingor, D. Schild and T. Salditt, "X-ray phase contrast tomography from whole organ down to single cells", Proc. SPIE 9212, 92120R (2014).

- M. Krenkel, M. Bartels, and T. Salditt, "Transport of intensity phase reconstruction to solve the twin image problem in holographic x-ray imaging", Optics Express 21, 2220 (2013).

- M. Bartels, M. Krenkel, J. Haber, R. N. Wilke, and T. Salditt "X-Ray Holographic Imaging of Hydrated Biological Cells in Solution”, Phys. Rev. Lett. 114, 048103 (2015)

- M. Bartels, M. Krenkel, P. Cloetens, W. Möbius, and T. Salditt "Myelinated mouse nerves studies by X-ray phase contrast zoom tomography", J. Struct. Biol., in press (2015)

- M. Töpperwien, M. Krenkel, T. Salditt and B. Eggart "X-ray sCMOS camera with on-chip scintillator enables fast phase-contrast tomography", Hamamatsu Photonics Europe GmbH, Application Note (2015)

- T. Salditt, M. Osterhoff, M. Krenkel, R. N. Wilke, M. Priebe, M. Bartels, S. Kalbfleisch and M. Sprung "Compound focusing mirror and X-ray waveguide optics for coherent imaging and nano-diffraction", J. Synchrotron Rad. 22, 867-878 (2015).

- R. N. Wilke, M. Hoppert, M. Krenkel, M. Bartels and T. Salditt "Quantitative X-ray phase contrast waveguide imaging of bacterial endospores" J. Appl. Cryst. 48, 464-476 (2015)

- C. Dullin, S. dal Monego, E. Larsson, S. Mohammadi, M. Krenkel, C. Garrovo, S. Biffi, A. Lorenzon, A. Markus, J. Napp, T. Salditt, A. Accardo, F. Alves, G. Tromba "Functionalized synchrotron in-line phase-contrast computed tomography: a novel approach for simultaneous quantification of struc- 
tural alterations and localization of barium-labelled alveolar macrophages within mouse lung samples" J. Synchrotron Rad.22, 143 (2015)

- A. Ruhlandt, M. Krenkel, M. Bartels, and T. Salditt "Three-dimensional phase retrieval in propagation-based phase-contrast imaging", Phys. Rev. A. 89, 033847 (2014).

- M. Bartels, V. H. Hernandez, M. Krenkel, T. Moser and T. Salditt "Phase contrast tomography of the mouse cochlea at microfocus x-ray sources", Appl. Phys. Lett. 103, 083703 (2013).

- C. Olendrowitz, M. Bartels, M. Krenkel, A. Beerlink, R. Mokso, M. Sprung and T. Salditt, "Phase-contrast x-ray imaging and tomography of the nematode Caenorhabditis elegans", Phys. Med. Biol. 57, 5309 (2012). 


\section{Danksagung}

An dieser Stelle möchte ich allen danken, die zum erfolgreichen Gelingen dieser Arbeit beigetragen haben.

Mein ganz besonderer Dank gilt Prof. Dr. Tim Salditt für die ausgezeichnete Betreuung und die Ermöglichung dieser Arbeit am Institut für Röntgenphysik. Besonders für das bereits zu Beginn meines Masterstudiums entgegengebrachte Vertrauen und die Freiheit, Messzeiten und Laborexperimente entscheidend mitzugestalten möchte ich mich bedanken. Durch die vielen fachlichen Diskussionen, Erläuterungen sowie seine Begeisterung für die Wissenschaft wurde meine Motivation stets gefördert und eine entspannte und angenehme Arbeitsatmosphäre geschaffen.

Ich bedanke mich bei Prof. Dr. Detlev Schild und PD Dr. Timo Aspelmeier für die Betreuung meiner Arbeit im Rahmen des Thesis Committees und für die Korreferenz meiner Doktorarbeit. Außerdem möchte ich mich bei Prof. Dr. Jens Frahm und Dr. Florian Rehfeldt für die Beiteiligung an meiner Prüfungskommission bedanken. Weiterhin gilt mein ganz herzlicher Dank Prof. Dr. Frauke Alves, nicht nur für das Mitwirken in der Prüfungskommission sondern auch für die Ermöglichung der stets sehr interessanten Kollaborationen, die einen Großteil der Ergebnisse dieser Arbeit ermöglicht haben. Insbesondere gilt mein Dank dabei auch Dr. Andrea Markus, Christian Dullin und Bärbel Heidrich für die aufschlussreichen Diskussionen und die Planung und Analyse verschiedener biomedizinisch relevanter Experimente.

Ein ganz besonderes Dankeschön geht an Dr. Matthias Bartels, der durch seine Begeisterung für die experimentelle und algorithmische Entwicklung mein Interesse an den Themen dieser Doktorarbeit stark gefördert hat. Insbesondere fachliche Diskussionen und die Zusammenarbeit während diverser Messzeiten haben in entscheidender Weise die Qualität der Experimente geprägt. Bei Mareike Töpperwien möchte ich mich ganz herzlich für viele aufschlussreiche Diskussionen sowie eine sehr angenehme Zusammenarbeit in vielerlei Hinsicht bedanken. Vor allem ihr Einsatz während gemeinsamer Messzeiten und ein guter Blick für die relevanten Dinge waren ein wichtiger Faktor, um qualitativ hochwertige Ergebnisse zu 
erzielen. Ich danke Aike Ruhlandt für zahlreiche fachliche Diskussionen, für eine angenehme Atmosphäre im Büro und nicht zuletzt für die Hilfe in der algorithmischen Umsetzung.

Ein ganz besonderer Dank gilt auch Dr. Robin Wilke und Johannes Hagemann für die vielen aufschlussreichen Diskussionen. Ich danke auch Malte Vaßholz, der durch sein technisches Verständnis viele Experimente stark bereichert hat. Weiterhin möchte ich mich bei Jan Goeman und Dr. Markus Osterhoff für die Unterstützung in allen IT-Angelegenheiten bedanken. Mein Dank gilt auch Simon Maretzke, der mir die Grundlagen des Gauß-Newton Verfahrens nähergebracht hat und die Anpassung des Algorithmus für die Toolbox vorgenommen hat. Außerdem danke ich der Gruppe von Prof. Dr. Thorsten Hohage für die Umsetzung Bereitstellung des Gauß-Newton Verfahrens. Ich bedanke mich bei Dr. Klaus Giewekemeyer, der mein Interesse im Bereich der Phasenrekonstruktion gestärkt hat.

Ich danke Sarah Hoffmann und Johann Haber für die Weiterentwicklung der gebondeten Röntgenwellenleiter, auf dessen Grundlage viele Ergebnisse dieser Arbeit beruhen. Weiterhin danke ich Bastian Hartman und Peter Nieschalk ganz herzlich, für die Unterstützung bei mechanischen Arbeiten für den Laboraufbau und insbesondere für die meist sehr kurzfristige Umsetzung bei nahenden Messzeiten. Danke auch an Jochen Herbst und Mike Kanbach für zahlreiche Hilfen bei technischen Fragen und Probenpräparationen. Ich danke Kristin Müller, Bärbel Heidrich und Julia Scherber für Präparationen von Makrophagen. Ein ganz besonderer Dank gilt hier auch Marten Bernhardt, der mir stets bei biochemisch relevanten Fragen helfen konnte und mir die Arbeit mit Zellen nähergebracht hat. Außerdem danke ich Andrea Markus für die Arbeit mit den Mäusen, die Präparation der Lungen und nicht zuletzt für das Korrekturlesen dieser Arbeit.

Ein ganz besonderer Dank geht an Dr. Michael Sprung und sein Team. Sein Engagement und die zahlreichen Vorschläge und Ideen haben die Weiterentwicklung des GINIX Messplatzes wesentlich beeinflusst. Ich danke Dr. Peter Cloetens, Dr. Alexandra Pacureanu und Dr. Heikki Suhonen für die Unterstützung während der Messzeiten an der ESRF. Ich bedanke mich auch bei Sabine Balder und Kerstin Pluschke für die Hilfe bei vielen administrativen Aufgaben.

Ein großes Dankeschön auch an alle Mitarbeiter des Instituts für Röntgenphysik für die tolle Arbeitsatmosphäre.

Diese Forschungsarbeit wurde vom Bundesministerium für Bildung und Forschung unter der Fördernummer 05K10MGA und dem Sonderforschungsbereich 755 "Nanoscale Photonic Imaging" finanziert. 
Abschließend möchte ich mich ganz besonders bei meinen Eltern für ihre durchgehende Unterstützung und ihr Vertrauen und herzlichst bei Daniela Emken bedanken. 



\section{Lebenslauf}

Name: $\quad$ Martin Krenkel

Geburtsdatum: $\quad$ 08. August 1986

Geburtsort: Berlin

Nationalität: Deutsch

\section{Akademischer Werdegang}

seit 09/2012 Wissenschaftlicher Mitarbeiter am Institut für

Röntgenphysik, Georg-August-Universität Göttingen

08/2012 Master of Science, Titel der Arbeit: "Quantitative

Phasenkontrast-Mikrotomographie"

10/2010 - 08/2012 Studium der Physik, Georg-August-Universität Göttingen

09/2010 Bachelor of Science, Titel der Arbeit: "Suprafluidität in

Asymmetrischen Fermiflüssigkeiten - Phasenübergänge 2.

Ordnung"

10/2007 - 09/2010 Studium der Physik, Technische Universität Braunschweig

07/2006 - 04/2010 Grundwehrdienstleistender, Fliegerhorst Goslar

06/2006 Abitur, Christian-von-Dohm Gymnasium Goslar 